\title{
MOTION CONTROL OF NON-FIXED BASE ROBOTIC MANIPULATORS
}

By

\author{
Frederick Michael Carter
}

B. A. Sc. (Engineering Physics) University of British Columbia

\author{
A THESIS SUBMITTED IN PARTIAL FULFILLMENT OF \\ THE REQUIREMENTS FOR THE DEGREE OF \\ Master of Applied Science \\ in \\ THE FACULTY OF GRADUATE STUDIES \\ MECHANICAL ENGINEERING
}

We accept this thesis as conforming

to the required standard

THE UNIVERSITY OF BRITISH COLUMBIA

April 1997

(C) Frederick Michael Carter, 1997 
In presenting this thesis in partial fulfilment of the requirements for an advanced degree at the University of British Columbia, I agree that the Library shall make it freely available for reference and study. I further agree that permission for extensive copying of this thesis for scholarly purposes may be granted by the head of my department or by his or her representatives. It is understood that copying or publication of this thesis for financial gain shall not be allowed without my written permission.

Mechanical Engineering

The University of British Columbia

2075 Wesbrook Mall

Vancouver, Canada

V6T $1 \mathrm{Z} 4$

$\therefore$ Date:

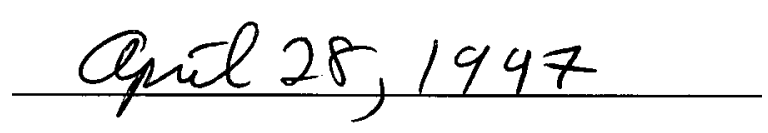




\begin{abstract}
Robotic manipulators mounted on spacecraft experience a number of kinematic, dynamic, and control problems because the motion of the spacecraft is affected by the robot motion. Because of this dynamic coupling, robot motion required to produce a given robot end-effector position for a fixed base manipulator would not result in correct end-effector position for the same manipulator mounted on a spacecraft. In this thesis, the general three dimensional equations of motion are derived for an $n$ link manipulator mounted on a non-fixed base object. Instead of performing a single inverse kinematic calculation at the beginning of a movement to determine the required joint setpoints, multiple inverse kinematic updates are done throughout a movement. The updating sequence is determined by an optimal inverse kinematic updating algorithm. This motion control algorithm is based on experimental simulation results performed in Matlab and a set of performance indices that are used as guidelines. Simple PD joint controllers are used for servoing the manipulator joints for a planar robot application. A joint trajectory generator utilizing velocity time scaling and quintic polynomials is developed. In addition to compensating for the base motion, it is shown that multiple updating requires less energy consumption than single inverse kinematic calculation based movements. Endpoint overcompensation and endpoint servoing are two techniques that enable any desired manipulator accuracy assuming kinematic and dynamic singuarities are not encountered. The derived motion control techniques incorporate the base motion without base motion control. Knowledge of the system dynamics is not required and the iterative inverse kinematics is performed online without model prediction.
\end{abstract}


Table of Contents

$\begin{array}{ll}\text { Abstract } & \text { ii }\end{array}$

List of Tables $\quad$ vi

List of Figures $\quad$ vii

$\begin{array}{lll}\text { Acknowledgement } & \mathbf{x}\end{array}$

Nomenclature $\quad$ xi

1 Introduction 1

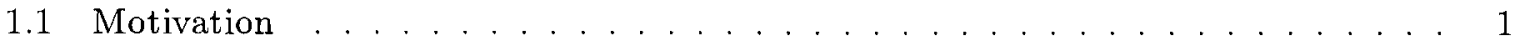

1.2 Objective of Motion Control Algorithms . . . . . . . . . . . . . . 3

1.3 Outline of Thesis Content . . . . . . . . . . . . . . 3

2 Literature Review 5

3 System Equations $\quad 8$

3.1 Manipulator and Base 3-D Configuration . . . . . . . . . . 8

3.2 Generalized Equations of Motion . . . . . . . . . . . . . . . 11

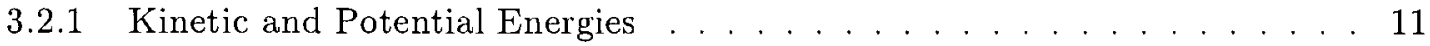

3.2 .2 Lagrange Equations . . . . . . . . . . . . . . . . . . 15

3.2 .3 Equation Expansion using MAPLE . . . . . . . . . . . . . . 20

3.2 .4 Equation Verification using MAPLE . . . . . . . . . . . . 22

3.3 Joint Trajectories . . . . . . . . . . . . . . . . . . . . . . . . 24

3.4 Joint Servo Control . . . . . . . . . . . . . . . . . . . 25 
4 Inverse Kinematics and Trajectory Generation

4.1 Review of Inverse Kinematics Methods . . . . . . . . . . . . . . . . . . 31

4.2 Fixed Base Inverse Kinematics . . . . . . . . . . . . . . . . . . . 32

4.3 Inverse Kinematics Updating Algorithm for Moving Base . . . . . . . . . . . 34

4.4 Joint Trajectory Updating . . . . . . . . . . . . . . . . . . . . 35

4.5 Joint Trajectory Velocity Time Scaling . . . . . . . . . . . . . . . . . 38

4.6 Joint Servo Control Specifications . . . . . . . . . . . . . . . . . . 39

4.6 .1 Effect of Torque Actuator Limits . . . . . . . . . . . . . . . 41

4.7 Space Manipulator Workspaces and Dynamic Singularities . . . . . . . . . . 45

5 Dynamic Simulation Results $\quad 48$

5.1 No Updates . . . . . . . . . . . . . . . . . . . . . . . . . . . . . . . . . . 48

5.2 Effect of Mass - Inertia Ratio . . . . . . . . . . . . . . . . 53

5.3 Effect of Total Movement Time and Time Scaling . . . . . . . . . . . 53

5.4 Effect of Single Updates . . . . . . . . . . . . . . . . . . . . 55

5.5 Effect of Multiple Updates and Update Frequency _ . . . . . . . . . . 65

5.6 Effect of Endpoint Overcompensation . . . . . . . . . . . . 70

5.7 Effect of Endpoint Servoing . . . . . . . . . . . . . . . 74

6 Inverse Kinematics Guidelines and Performance Indices $\quad 77$

6.1 Inverse Kinematics Updating Sequence Performance Indices . . . . . . . . . . 77

6.1 .1 Manipulator Energy Expended . . . . . . . . . . . . . . . 79

6.2 Guidelines for Inverse Kinematics Updating Sequences . . . . . . . . . . . . 80

6.3 Further Examples and Algorithm Verification . . . . . . . . . . . 87

7 Conclusion $\quad 94$

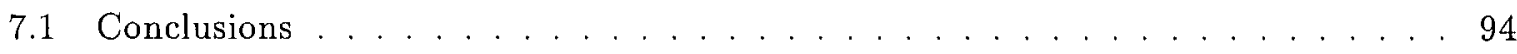

7.2 Recommendations for Future Work . . . . . . . . . . . . . . . 95 
B Matlab Files 


\section{List of Tables}

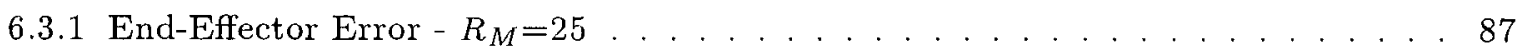

6.3 .2 End-Effector Error $-R_{M}=3 \ldots \ldots \ldots \ldots \ldots \ldots$

6.3 .3 End-Effector Error $-E_{o c} \ldots \ldots \ldots \ldots \ldots \ldots$ 


\section{List of Figures}

1.1 Robot-Base Coupling Position Error . . . . . . . . . . . . . . . . 2

3.1 Robot Configuration . . . . . . . . . . . . . . . . . . . . 9

3.2 Centre of Mass Moving - Wrong Equations . . . . . . . . . . . . . . . 23

3.3 Quintic Polynomial Position and Velocity . . . . . . . . . . . . . . 26

3.4 Torque Controller . . . . . . . . . . . . . . . . . . . . 30

4.1 Inverse Kinematics for 2-Link Robot . . . . . . . . . . . . . . . . 33

4.2 Single Joint Trajectory Update $-\theta_{1}$ vs. Time . . . . . . . . . . . . 36

4.3 Single Joint Trajectory Update $-\dot{\theta}_{1}$ vs. Time . . . . . . . . . . . . 36

4.4 Multiple Trajectory Updates $-\theta_{1}$ vs. Time . . . . . . . . . . . . 37

4.5 Multiple Trajectory Updates $-\dot{\theta}_{1}$ vs. Time . . . . . . . . . . . . 37

4.6 Velocity Time Scaling $-\theta_{1}$ vs. Time . . . . . . . . . . . . . . 39

4.7 Velocity Time Scaling $-\dot{\theta}_{1}$ vs. Time . . . . . . . . . . . . . . 40

4.8 Torque Limits $-\theta_{1}$ vs. Time . . . . . . . . . . . . . . . . . . 43

4.9 Torque Limits $-\dot{\theta}_{1}$ vs. Time . . . . . . . . . . . . . . . . . . 44

4.10 Torque Limits - Joint Torque vs. Time . . . . . . . . . . . . . . . . . . . 44

4.11 Space Manipulator Workspaces . . . . . . . . . . . . . . . . . . 47

5.1 Fixed and Moving Base System Trajectories . . . . . . . . . . . . . . . . 49

5.2 Fixed and Moving Base Joint Angle and Angular Velocity . . . . . . . . . . 50

5.3 Moving Base Position and Velocity . . . . . . . . . . . . . . . . 51

5.4 Moving Base Angles, Angular Velocities, System Trajectories . . . . . . . . . 52

5.5 End-Effector Trajectories - Variable Mass Ratio . . . . . . . . . . . . . . . . 54

5.6 Single Update End-Effector Trajectories . . . . . . . . . . . . . . . . . 56 
5.7 Single Update End-Effector Trajectories Close $U_{p} \ldots \ldots \ldots$

5.8 Single Update $\theta_{1}$ vs. Time . . . . . . . . . . . . . . . . . . 58

5.9 Single Update $\dot{\theta}_{1}$ vs. Time . . . . . . . . . . . . . . . . . . . 58

5.10 Single Update Joint 1 Torque vs. Time . . . . . . . . . . . . . . . . . . . 59

5.11 Single Update $\theta_{2}$ vs. Time . . . . . . . . . . . . . . . . . . . . 59

5.12 Single Update $\dot{\theta}_{2}$ vs. Time . . . . . . . . . . . . . . . 60

5.13 Single Update Joint 2 Torque vs. Time . . . . . . . . . . . . . . . . 60

5.14 Single Update Base Position and Velocity vs. Time . . . . . . . . . . . . . 61

5.15 Single Update Base Position and Velocity . . . . . . . . . . . . . 62

5.16 Single Update $\phi$ vs. Time . . . . . . . . . . . . . . . . . 63

5.17 Single Update $\dot{\phi}$ vs. Time . . . . . . . . . . . . . . . . . 63

5.18 Single Update End-Effector Error . . . . . . . . . . . . . . . . . . . 64

5.19 Multiple Update End-Effector Trajectories . . . . . . . . . . . . . . . . . 66

5.20 Multiple Update End-Effector Trajectories Close Up . . . . . . . . . . . . 66

5.21 Multiple Update $\theta_{1}$ vs. Time . . . . . . . . . . . . . . 67

5.22 Multiple Update $\dot{\theta}_{1}$ vs. Time . . . . . . . . . . . . . . . . . . 67

5.23 Multiple Update Joint 1 Torque vs. Time . . . . . . . . . . . . 68

5.24 Multiple Update End-Effector Error - 10\% Steps . . . . . . . . . . . . . 69

5.25 Multiple Update End-Effector Error - 5\% Steps . . . . . . . . . . . . . . . 69

5.26 Multiple Update End-Effector Error - 2.5\% Steps . . . . . . . . . . . 70

5.27 Multiple Update End-Effector Errors $-\mathrm{x}=0, \mathrm{y}=0 \ldots \ldots \ldots \ldots$

5.28 Multiple Update End-Effector Trajectory $-\mathrm{x}=0, \mathrm{y}=0 \ldots \ldots \ldots \ldots$

5.29 Multiple Update Angle and Angular Velocity - $\mathrm{x}=0, \mathrm{y}=0 \ldots \ldots \ldots$

5.30 Endpoint Overcompensation - System Movement . . . . . . . . . . 73

5.31 Endpoint Servoing - System Movement . . . . . . . . . . . . 75

5.32 Endpoint Servoing - Trajectory Closeup . . . . . . . . . . . . 76

6.1 Joint Angular Velocity and Torque - Multiple Updating . . . . . . . . . . 81 
6.2 Joint Angular Velocity and Torque -3 Single Movements . . . . . . . . . . . 82

6.3 Update Start Time vs. Mass Ratio . . . . . . . . . . . . . . . . . . 84

6.4 Update Factor vs. End-Effector Total Distance . . . . . . . . . . . . . . 85

6.5 Updating Sequence vs. Reference Sequence . . . . . . . . . . . . 88

6.6 System Movement - Five Endpoint Servos . . . . . . . . . . . . . . . . 91

6.7 System Movement - Manipulator Singularity . . . . . . . . . . . . . . . 92 


\section{Acknowledgement}

I would like to thank my supervisor Dr. Dale B. Cherchas for the guidance and expertise he provided in the development of this work, my parents Ted and Bette Carter for their encouragement throughout university, and my wife Lindy for her love and support always. I would also like to acknowledge my late grandfather, Herbert Heaton, who taught me that anything is possible if we set our minds on it. 


\section{Nomenclature}

\section{REFERENCE FRAMES :}

$F_{I}$ Inertial frame of manipulator - base system.

$F_{B}$ Base Frame located at the centre of mass of the base.

$F_{O}$ Zeroth coordinate frame or robot base coordinate frame.

$F_{1}$ Coordinate frame of the first link of the manipulator.

$F_{i}$ Coordinate frame of the $i$ th link of the manipulator. VECTORS (boldface):

P Position of frame $F_{B}$ relative to and projected onto frame $F_{I}$.

$\Phi$ Rotation of base in frame $F_{I}$.

$\mathbf{r}_{B}$ Position of frame $F_{O}$ relative to and projected onto frame $F_{B}$.

$\mathbf{r}_{I}$ Position of frame $F_{O}$ relative to and projected onto frame $F_{I}$.

$\mathbf{d}_{i}$ Position of frame $F_{i}$ relative to and projected onto frame $F_{O}$.

$\mathbf{r}_{i}$ Position of point on link $i$ relative to and projected onto frame $F_{O}$.

$\rho_{i}$ Position of point on link $i$ relative to and projected onto frame $F_{i}$.

$\mathbf{v}_{i}$ Velocity of point on link $i$ relative to and projected onto frame $F_{I}$.

b Position of point on the base relative to and projected onto frame $F_{B}$.

$\mathrm{b}_{I}$ Position of point on the base relative to and projected onto frame $F_{I}$. 
$\mathrm{v}_{I B}$ Velocity of point on the base relative to and projected onto frame $F_{I}$.

g Gravity vector.

w Angular velocity of the base.

$\tau$ Generalized torque vector.

Q Generalized state vector.

q Inverse kinematic joint parameters.

$\mathbf{k}_{\text {step }}$ Linear sequence of update times.

$V_{j}$ Maximum joint velocities.

$\tau_{j}$ Maximum joint torques.

$S_{u}$ Update sequence during a manipulator movement.

$S_{\text {updseqoriginal }}($ lastvalue) Original update sequence before stepsize rounding. MATRICES (boldface) :

$\mathbf{H}_{(i, j)}$ Homogeneous transformation from the $j$ th frame to the $i$ th frame.

$\mathrm{H}_{(I, B)}$ Homogeneous transformation from the $B$ th frame to the $I$. th frame.

$\mathbf{H}_{(B, 0)}$ Homogeneous transformation from the Zeroth frame to the $B$ th frame.

$\mathbf{H}_{(0,1)}$ Homogeneous transformation from the first frame to the zeroth frame.

$\mathbf{H}_{(0, i)}$ Homogeneous transformation from the $i$ th frame to the zeroth frame.

$\mathbf{T}_{6}$ Desired end-effector position or transformation matrix from the sixth frame to the zeroth frame.

$\mathbf{J}_{i}$ Pseudo inertia matrix of link $i$. 
$\mathbf{J}_{B}$ Pseudo inertia matrix of the base.

M Manipulator inertia matrix.

V Coriolis - Centripetal matrix.

G Gravity matrix.

F Disturbance term matrix.

SCALARS :

$z_{i}$ Generalized coordinates for the base.

$q_{i}$ Generalized coordinates for the links.

$\theta$ Coordinate for a rotational link.

$d$ Coordinate for a prismatic link.

$d m$ Particle of mass.

$K_{i}$ Kinetic energy of link $i$.

$K_{L}$ Kinetic energy of the links.

$K_{B}$ Kinetic energy of the base.

$K_{S}$ Kinetic energy of the system.

I Mass moment of inertia.

$P_{i}$ Potential energy of the link $i$.

$P_{B}$ Potential energy of the base.

$P_{S}$ Total potential energy of the system.

$F_{i}$ Generalized force or torque. 


\section{$L$ Lagrangian function.}

$c_{i}^{d}$ Quintic trajectory.

$c_{0}$ Quintic trajectory initial condition.

$c_{f}$ Quintic trajectory final condition.

$q_{d}(t)$ Desired joint trajectory.

$e(t)$ Tracking error of a joint variable.

$u(t)$ Control signal.

$K_{p}$ Proportional controller gain.

$K_{v}$ Derivative controller gain.

$K_{I}$ Integral controller gain.

$\tau_{i}$ Torque applied at joint $i$.

$\varepsilon$ Integral of $e(t)$.

$s$ Laplace Transform complex frequency variable.

$\zeta_{n}$ Damping coefficient.

$\omega_{n}$. Joint natural frequency.

$\omega_{r}$ Link structural natural frequency.

$k_{r}$ Link structural stiffness.

$J_{r}$ Link structural inertia.

$\tau_{\text {update }}$ Update error torque.

$l_{i}$ Length of link $i$. 
$m_{i}$ Mass of link $i$.

$\tau_{i_{\max }}$ Joint $i$ maximum actuator torque.

$A C C_{e}$ Accuracy of the end-effector in the inertial frame.

$J_{\text {energy }}$ Energy expended by a joint during a movement.

$E N_{j}$ Energy expended by a manipulator during a movement.

$N U M_{u}$ Number of updates.

$D I S T_{b}$ Base disturbance during movement.

$R_{M}$ Base-manipulator mass ratio.

$\Delta_{e}$ Total required end-effector movement distance.

$T_{u}$ Time during a manipulator movement when an update is performed.

$E_{o c}$ Endpoint overcompensation.

$E P$ Endpoint servoing at the end of a movement.

$T_{\text {total }}$ Total movement time.

$U_{\text {time }}$ Movement start time, percentage of $T_{\text {total }}$.

$k_{U_{\text {time }}}$ Update time scale factor.

coef Coefficient in the update sequence equation.

$k_{\text {stepsize }}$ Average update sequence step size.

$N_{k}$ Number of updates in an updating sequence. 


\title{
Chapter 1
}

\author{
Introduction
}

\subsection{Motivation}

Most robotic manipulators can be considered to have a base fixed in an inertial frame. There are, however, manipulators for which this is not the case. Two examples would be manipulators mounted on free flying-satellites or submarines. In such cases, the commanded arm motions required to produce a given robot end-effector position (for grasping), calculated assuming a fixed base, will result in position error of the end effector because of the dynamic coupling between the robot and base as shown in Figure 1.1. This position error increases as the mass (and inertia) ratio of the base to manipulator decreases and as the end-effector to desired point distance increases. This produces a non-classical robot kinematics problem since the relationship between the joint coordinates and the end-effector position is no longer a purely kinematic one, but is described by a set of non-linear coupled differential equations. One way to compensate for the base rotation in a space vehicle is to mount stabilizing devices such as gyroscopes in the vehicle base and drive them so as to negate the reaction moments that are transmitted to the robot base. To accomplish this one has to measure, on-line, the disturbing moments being transmitted to the base. In the same way thrusters can be used to negate the translational motion of the base. One study, [Long 87], showed that if only gyroscopes were used to negate the rotation, the translational movement could be compensated for using modified inverse kinematics equations without thrusters or control jets. Other studies have demonstrated that by 


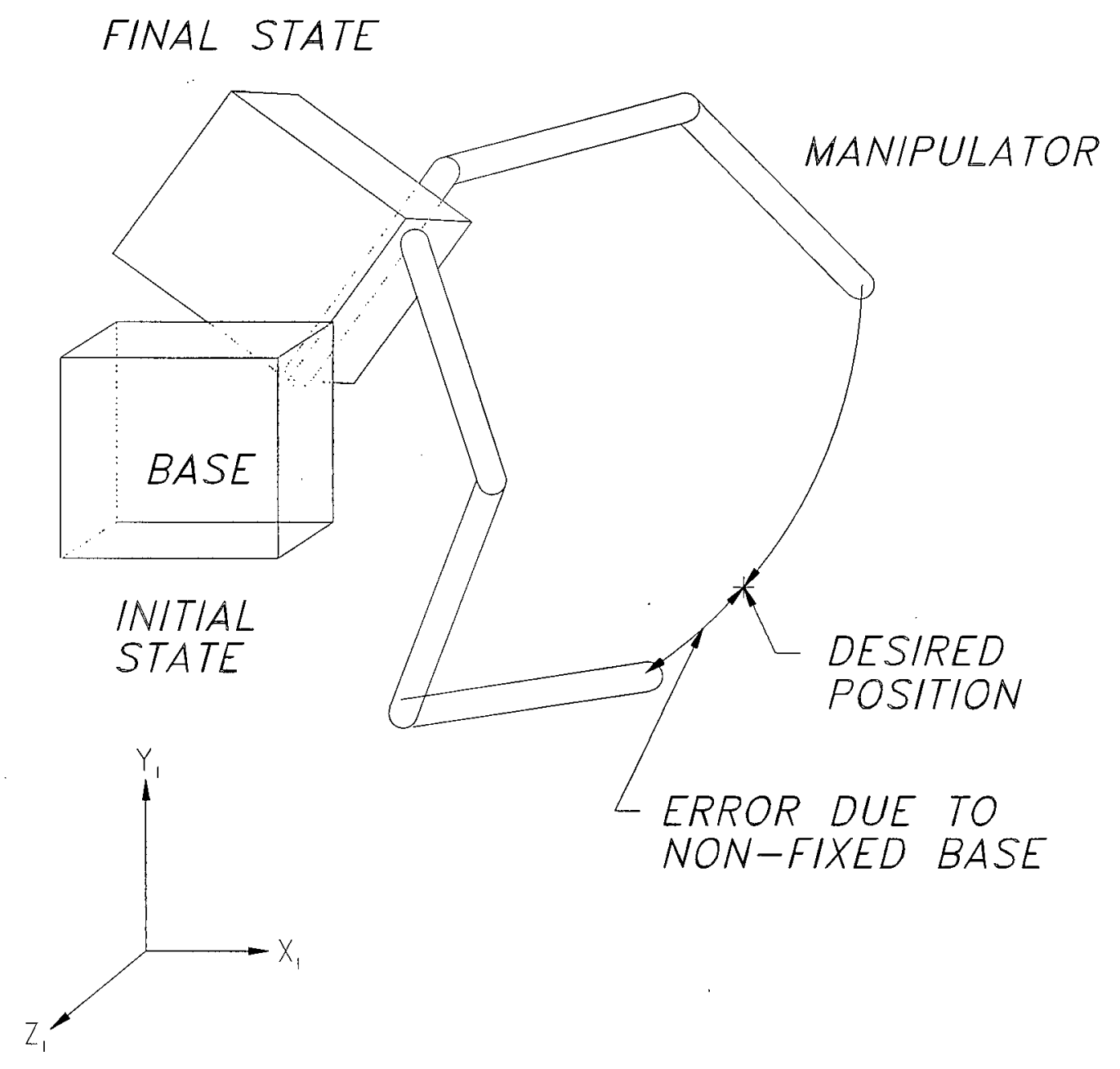

Figure 1.1: Robot-Base Coupling Position Error 
decoupling robot motion from satellite motion an end-effector can track a desired reference while slow gross positioning by a control jet is used for the satellite. One problem with vehicle base stabilization, e.g., using gyroscopes or control jets for spacecraft control, is that excessive use of attitude control fuel can degrade the system life of the spacecraft. Some early studies of space manipulators have neglected the dynamic coupling, assuming there will be a human present to visually adjust for the errors. Two papers, [Fu 87], [Umet 87], discussed methods where optimal inverse kinematics solutions are determined using optimal control methods. These are very effective algorithms but they require all the dynamic parameters of the system to be known. This is an impractical requirement when the parameters are not known exactly as in the case of a previously unmeasured payload mass.

\subsection{Objective of Motion Control Algorithms}

In this thesis, a motion control technique based on multiple inverse kinematic updating is developed. By updating the manipulator inverse kinematic solutions throughout the motion, the requirement for attitude servoing can be eliminated. The dynamic parameters of the system need not be known but if they are known approximately, the algorithm performance can be further improved. The only kinematic quantities required are the orientation and position of the base relative to the desired end effector position at certain points during a movement. Such quantities are measureable, for example, by cameras mounted on the space vehicle or the manipulator.

\subsection{Outline of Thesis Content}

The work presented in the thesis is arranged as follows. Chapter 2 reviews previous related work in the area of non-fixed base robotics. Chapter 3 presents the configuration of a robot 
on a moving base and develops the equations of motion describing this configuration using the Lagrange method for use in the simulations. Joint trajectories and joint servo control are also covered. The use of the symbolic algebra package Maple for expanding and verifying the system equations of motion is described. In Chapter 4, the difference between fixed base and non-fixed base inverse kinematic methods are discussed. The inverse kinematics updating algorithm, joint trajectory updating, and joint velocity time scaling techniques are developed. A discussion of dynamic singularities that occur in space manipulator systems, and their effect on the workspace of the manipulator is presented. In Chapter 5, dynamic simulations for a 2-D system are performed. The variables that effect the desired end-effector error for a moving base system are categorized. Endpoint overcompensation and endpoint servoing, two methods for reducing end-effector error, are explained. In Chapter 6 a set of performance indices and guidelines for the updating algorithm, based on the results of Chapter 5, are developed analytically. These indices serve to automate the updating algorithm, so that a specified manipulator accuracy can be achieved for any manipulator-base system. This is assuming kinematic or dynamic singularities are not encountered. Further examples (simulations) are performed to verify the validity of the algorithm. Chapter 7 presents conclusions for this work and suggestions for future work. 


\section{Chapter 2}

\section{Literature Review}

The dynamic coupling that occurs in a moving base robot manipulator system has not been extensively studied until comparatively recently. Early studies in this field neglected the dynamic coupling between the robot and the base ([Bron 86]; [Fren 85]; [Lee 85]). One of the first approaches to consider coupling was formulated in [Long 87]. In this work it was assumed the orientation of the spacecraft could be kept constant by using gyroscopes to negate the torques being transmitted at the base. The holonomic conservation of momentum principle was then used to eliminate the remaining three translational degrees of freedom. Modified inverse kinematics and dynamic equations in closed form for a spacecraft with a spherical polar-coordinate manipulator were derived. A study in [Vafa 90] introduced a new' method, the Virtual Manipulator Approach. In this approach, constant spacecraft orientation is also assumed. The Virtual Manipulator can be considered to be in a fixed inertial frame that uses the center of mass of the system as a Virtual Ground. The position of the Virtual Ground is calculated at the start of each movement and is invariant as long as the system mass properties do not change. The inverse kinematics solution is then found using the holonomic conservation of momentum principle. One problem with these methods is that energy is consumed in order to keep the spacecraft orientation constant. However, in [Vafa 90] it was assumed that renewable photovoltaic power was being used. Another complication of the Virtual Manipulator approach is the fact that the center of mass of the system and payload must be accurately calculated to achieve

accurate inverse kinematic results. Determining the dynamic parameters of a system can be 
very difficult. One example would be if an unknown satellite was picked up part way through a dynamic movement. The center of mass of the system will change (and the dynamic coupling will increase). A later paper [Dubo 91] describes a new tool called the Enhanced Disturbance Map (EDM) that is used in conjunction with the Virtual Manipulator approach. Using the EDM, the inverse kinematics solution can be determined for the spacecraft/manipulator system that minimizes the disturbance forces on the spacecraft and hence minimizes the necessary fuel for spacecraft relocation. In one paper, [Xu 93], a measure or performance index was defined that characterizes the degree of dynamic coupling between a spacecraft and manipulator given a certain trajectory. This measure can then be used to optimize robot trajectories to minimize base disturbances.

Some studies [Alex 87], [Umet 87] have demonstrated that when the mass of the base or spacecraft is relatively large compared to that of the manipulator, decoupling between the endeffector motion and the spacecraft motion can be done. Using feedforward compensation from the satellite forces and torques, a feedback control scheme can be used to give approximate results. One important result discussed in [Umet 87] is that linear momentum conservation is integrable and hence a holonomic constraint. Angular momentum conservation though is non-integrable and hence a non-holonomic constraint. Several approaches have tried to control the end-effector position accurately while using satellite thruster - gyroscope positioning for large slow movements. The scenario of a small, high bandwidth manipulator mounted on a larger, slow bandwidth manipulator (macro/micro manipulator systems) is similar. This type of control was studied in [Sali 85], [Egel 87], and [Khat 88]. A spacecraft/manipulator study in [Egel 93] proposed a control scheme that decoupled end-effector motion and total system momentum. Using the augmented task-space approach, recursive formulations of the kinematic and dynamic equations are performed, and a kinematic redundancy resolution scheme is used to achieve a solution. In the same paper, the manipulator end-effector tracked a desired reference and a slow gross positioning was used for the satellite. Unfortunately, the satellite repositioning uses fuel. 
One paper, [Fern 92], discusses attitude control of spacecraft/manipulator systems using internal (joint) motions for control. First, the controllability of the system is discussed and proven. Then a "Basis Algorithm For Near Optimal Solutions" is formulated for Non-holonomic Motion Planning (NMP) systems. This approach yields an approximate optimal solution that minimizes the control inputs (energy required). In another paper, [Nags 92]), a computational scheme is proposed that uses the kinematic conditions between adjoined links and their derivatives with respect to time along with the link linear and angular velocities and accelerations. These velocities and accelerations are separated into two parts, one that depends on the satellite motion and one that is determined by a recursive algorithm. Using conservation laws and torque control to determine the satellite linear and angular velocities, the kinematics and inverse dynamics of a satellite based manipulator can then be calculated. These are very effective algorithms but they require that all the dynamic parameters of the system are known.

Unlike singularities for fixed-base manipulators that are solely a function of kinematics, space manipulators without base motion control encounter dynamic singularities. These singularities occur when the end-effector cannot move in some inertial direction, and are a function of the dynamic properties of the manipulator and base. Dynamic singuarities occur when the system generalized Jacobian, $J^{*}$ as defined in [Papa 93], becomes rank deficient or non-invertible. The Jacobian for a manipulator-base system relates the end-effector's linear and angular velocities in inertial space, to the controlled manipulator joint angles. Unlike fixed-base Jacobians, this generalized Jacobian depends upon dynamic parameters as well as kinematic ones. These principles are developed by E. Papadopoulus and S. Dubowsky, and can be found in [Papa 93] and [Papa 90]. Also discussed in [Papa 93] is the effect that these dynamic singularities have on the manipulators workspace. 


\section{Chapter 3}

\section{System Equations}

\subsection{Manipulator and Base 3-D Configuration}

The configuration of the manipulator and non-fixed base is as shown in Figure 3.1. In 3 dimensions, the base has 6 degrees of freedom and the robot $n$ degrees of freedom, one for each link. This gives the system $6+n$ degrees of freedom or one degree of freedom for each generalized coordinate. From Figure 3.1, we define the frames, matrices, and vectors in terms as shown in the list below.

Reference Frames :

$F_{I}$ Inertial frame of manipulator - base system.

$F_{B}$ Base Frame located at the centre of mass of the base.

$F_{O}$ Zeroth coordinate frame or robot base coordinate frame.

$F_{1}$ Coordinate frame of the first link of the manipulator.

$F_{i}$ Coordinate frame of the $i$ th link of the manipulator.

Matrices and Vectors:

$\mathbf{H}_{(i, j)}$ Homogeneous transformation from the $j$ th frame to the $i$ th frame. 


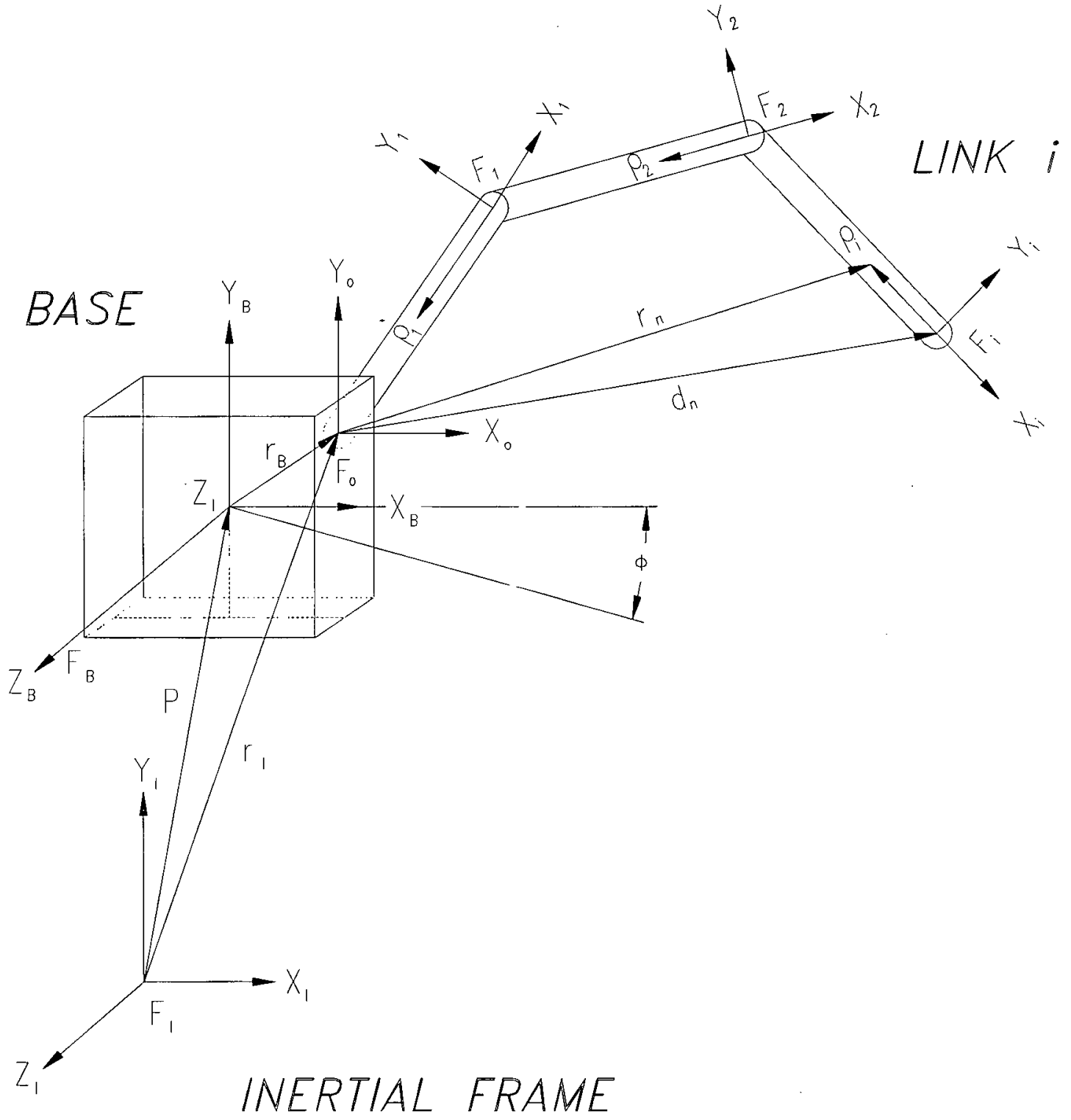

Figure 3.1: Robot Configuration 
$\mathbf{H}_{(I, B)}$ Homogeneous transformation from the $B$ th frame to the $I$ th frame.

$\mathbf{H}_{(B, 0)}$ Homogeneous transformation from the Zeroth frame to the $B$ th frame.

$\mathbf{H}_{(0,1)}$ Homogeneous transformation from the first frame to the zeroth frame.

$\mathbf{H}_{(0, i)}$ Homogeneous transformation from the $i$ th frame to the zeroth frame.

$\mathrm{P}$ Position of frame $F_{B}$ relative to and projected onto frame $F_{I}$.

$\Phi$ Rotation of base in frame $F_{I}$.

$\mathbf{r}_{B}$ Position of frame $F_{O}$ relative to and projected onto frame $F_{B}$.

$\mathbf{r}_{I}$ Position of frame $F_{O}$ relative to and projected onto frame $F_{I}$.

$\mathbf{d}_{i}$ Position of frame $F_{i}$ relative to and projected onto frame $F_{O}$.

$\mathbf{r}_{i}$ Position of point on link $i$ relative to and projected onto frame $F_{O}$.

- $\rho_{i}$ Position of point on link $i$ relative to and projected onto frame $F_{i}$.

$\mathbf{v}_{i}$ Velocity of point on link $i$ relative to and projected onto frame $F_{I}$.

b Position of point on the base relative to and projected onto frame $F_{B}$.

$\mathbf{b}_{B}$ Position of point on the base relative to and projected onto frame $F_{I}$.

$\mathbf{v}_{B}$ Velocity of point on the base relative to and projected onto frame $F_{I}$.

Homogeneous transformation matrices $\left(\mathbf{H}_{(,)}\right)$are $4 \times 4$ matrices which map position vectors expressed in homogeneous coordinates from one coordinate frame to another. They consist of a rotation submatrix and a position vector, along with scaling and perspective information. The generalized coordinate for each link will be denoted as $q_{i}$. If a link is revolute or prismatic $q_{i}$ is represented by either $\theta_{i}$ or $d_{i}$ respectively. The homogeneous matrix for a link will reflect whether the link is either translational or rotational. The translational coordinates of the base 
are defined as $\mathbf{P}=\left(P_{1}, P_{2}, P_{3}, 1\right)^{T}$. This corresponds to translations along the $\mathrm{X}, \mathrm{Y}$, and $\mathrm{Z}$ axes in the $\mathrm{F}_{I}$ frame. The rotational coordinates are defined as $\boldsymbol{\Phi}=\left(\Phi_{1}, \Phi_{2}, \Phi_{3}\right)$. These form an Euler angle system, known as a 3-2-3 system, which correspond to the following current frame rotational sequence:

1. A rotation of $\Phi_{1}$ about the $\mathrm{Z}$ axis in frame $F_{B}$.

2. A rotation of $\Phi_{2}$ about the new rotated $\mathrm{Y}$ axis in the rotated frame $F_{B}^{\prime}$.

3. A rotation of $\Phi_{3}$ about the new rotated $\mathrm{Z}$ axis in the rotated frame $F_{B}^{\prime \prime}$.

The generalized coordinate for each d.o.f. of the base will be denoted as $z_{s}$.

\subsection{Generalized Equations of Motion}

The equations of motion of the system are derived using the Lagrange Method. The kinetic energy for the base and the links will be derived. The potential energy of the base and links is ignored since it is assumed that the system is in space.

\subsubsection{Kinetic and Potential Energies}

Refering to Figure 3.1, the position of a point on link $i$ relative to and projected onto frame $F_{I}$ is

$$
\mathbf{r}_{i}=\mathbf{H}_{(I, B)} \mathbf{H}_{(B, 0)} \mathbf{H}_{(0,1)} \mathbf{H}_{(1,2)} \ldots \mathbf{H}_{(i-1, i)} \rho_{i}
$$

or simplifying,

$$
\mathbf{r}_{i}=\mathbf{H}_{(I, B)} \mathbf{H}_{(B, 0)} \mathbf{H}_{(0, i)} \rho_{i}
$$


The velocity of a point on link $i$ is then

$$
\mathbf{v}_{i}=\frac{d\left(\mathbf{r}_{i}\right)}{d t}
$$

or performing the differentiation,

$$
\begin{array}{r}
\mathbf{v}_{i}=\frac{d\left(\mathbf{H}_{(I, B)}\right)}{d t} \mathbf{H}_{(B, 0)} \mathbf{H}_{(0, i)} \rho_{i}+\mathbf{H}_{(I, B)} \frac{d\left(\mathbf{H}_{(B, 0)}\right)}{d t} \mathbf{H}_{(0, i)} \rho_{i}+ \\
\mathbf{H}_{(I, B)} \mathbf{H}_{(B, 0)} \frac{d\left(\mathbf{H}_{(0, i)}\right)}{d t} \rho_{i}+\mathbf{H}_{(I, B)} \mathbf{H}_{(B, 0)} \mathbf{H}_{(0, i)} \frac{d \rho_{i}}{d t}
\end{array}
$$

It should be noted that all the homogeneous matrices are functions of joint coordinates which are time dependent $\left(\right.$ eg. $\left.\mathbf{H}_{(I, B)}(\theta(t))\right)$. If we assume the links are rigid we obtain

$$
\frac{d \rho_{i}}{d t}=0
$$

The base is also assumed rigid so we can write

$$
\frac{d\left(\mathrm{H}_{(B, 0)}\right)}{d t}=0
$$

Since $\mathbf{H}_{(I, B)}$ is a function of the six base generalized coordinates $z_{s}$, and $\mathbf{H}_{(0, i)}$ is a function of the $i$ th link generalized coordinate, we write Equation 3.4 for compactness as

$$
\dot{\mathbf{r}}_{i}=\mathbf{v}_{i}=\sum_{s=1}^{6} \frac{\partial \mathbf{H}_{(I, B)}}{\partial \mathbf{z}_{s}} \mathbf{H}_{(B, 0)} \mathbf{H}_{(0, i)} \rho_{i} \dot{z}_{s}+\sum_{j=1}^{i} \mathbf{H}_{(I, B)} \mathbf{H}_{(B, 0)} \frac{\partial \mathbf{H}_{(0, i)}}{\partial \mathbf{q}_{j}} \rho_{i} \dot{q}_{j}
$$

Knowing that the velocity squared of a vector is

$$
\left(\frac{d \mathbf{r}_{i}}{d t}\right)^{2}=\operatorname{trace}\left(\dot{\mathbf{r}} \dot{\mathbf{r}}^{T}\right)
$$

we can write the kinetic energy of a particle of mass $d m$ on link $i$ at $\rho_{i}$ as

$$
d K_{i}=\frac{1}{2} \operatorname{trace}\left(\dot{\mathbf{r}} \dot{\mathbf{r}}^{T}\right) d m
$$

or, substituting Equation 3.9 into Equation 3.7 and multiplying we obtain

$$
d K_{i}=\frac{1}{2} \operatorname{tr}\left[\sum_{s=1}^{6} \sum_{j=1}^{i} \frac{\partial \mathbf{H}_{(I, B)}}{\partial \mathbf{z}_{s}} \mathbf{H}_{(B, 0)} \mathbf{H}_{(0, i)} \rho_{i} \rho_{i}^{T} \frac{\partial \mathbf{H}_{(0, i)}^{T}}{\partial \mathbf{q}_{j}} \mathbf{H}_{(B, 0)}^{T} \mathbf{H}_{(I, B)}^{T}\right] \dot{z}_{s} \dot{q}_{j} d m+
$$




$$
\begin{gathered}
\frac{1}{2} \operatorname{tr}\left[\sum_{s=1}^{6} \sum_{j=1}^{i} \frac{\partial \mathbf{H}_{(I, B)}}{\partial \mathbf{z}_{s}} \mathbf{H}_{(B, 0)} \mathbf{H}_{(0, i)} \rho_{i} \rho_{i}^{T} \frac{\partial \mathbf{H}_{(0, i)}^{T}}{\partial \mathbf{q}_{j}} \mathbf{H}_{(B, 0)}^{T} \mathbf{H}_{(I, B)}^{T}\right] \dot{z}_{s} \dot{q}_{j} d m+ \\
\frac{1}{2} \operatorname{tr}\left[\sum_{s=1}^{6} \sum_{j=1}^{i} \frac{\partial \mathbf{H}_{(I, B)}}{\partial \mathbf{z}_{s}} \mathbf{H}_{(B, 0)} \mathbf{H}_{(0, i)} \rho_{i} \rho_{i}^{T} \frac{\partial \mathbf{H}_{(0, i)}^{T}}{\partial \mathbf{q}_{j}} \mathbf{H}_{(B, 0)}^{T} \mathbf{H}_{(I, B)}^{T}\right] \dot{z}_{s} \dot{z}_{t} d m+ \\
\frac{1}{2} \operatorname{tr}\left[\sum_{s=1}^{6} \sum_{j=1}^{i} \frac{\partial \mathbf{H}_{(I, B)}}{\partial \mathbf{z}_{s}} \mathbf{H}_{(B, 0)} \mathbf{H}_{(0, i)} \rho_{i} \rho_{i}^{T} \frac{\partial \mathbf{H}_{(0, i)}^{T}}{\partial \mathbf{q}_{j}} \mathbf{H}_{(B, 0)}^{T} \mathbf{H}_{(I, B)}^{T}\right] \dot{q}_{j} \dot{q}_{k} d m
\end{gathered}
$$

The total kinetic energy of the links is given by integrating over the mass for each link and then summing as follows

$$
K_{L}=\sum_{i=1}^{n} K_{i}=\sum_{i=1}^{n} \int d K_{i}
$$

We also know that the integral of the term $\rho_{i} \rho_{i}^{T}$ over link $i$ is termed the pseudo inertia matrix

$$
\mathbf{J}_{i}=\int_{\text {linki }} \rho_{i} \rho_{i}^{T} d m
$$

For a 3-D rigid body the pseudo inertia matrix can be written as

$$
\mathbf{J}_{i}=\left(\begin{array}{cccc}
\int x_{i}^{2} d m & \int x_{i} y_{i} d m & \int x_{i} z_{i} d m & \int x_{i} d m \\
\int x_{i} y_{i} d m & \int y_{i}^{2} d m & \int y_{i} z_{i} d m & \int y_{i} d m \\
\int x_{i} z_{i} d m & \int y_{i} z_{i} d m & \int z_{i}^{2} d m & \int z_{i} d m \\
\int x_{i} d m & \int y_{i} d m & \int z_{i} d m & \int d m
\end{array}\right)
$$

where $I_{x x}$, the mass moment of inertia is written as

$$
I_{x x}=\iiint \mathbf{y}_{i}^{2}+\mathbf{z}_{i}^{2} \rho_{i} d x d y d z
$$

Combining terms and using the fact that $\operatorname{trace}(A)=\operatorname{trace}\left(A^{T}\right)$, we obtain the total kinetic energy of the manipulator as

$$
\begin{aligned}
K_{L}= & \sum_{i=1}^{n} \sum_{s=1}^{6} \sum_{j=1}^{i} \operatorname{tr}\left[\frac{\partial \mathbf{H}_{(I, B)}}{\partial \mathbf{z}_{s}} \mathbf{H}_{(B, 0)} \mathbf{H}_{(0, i)} \mathbf{J}_{i} \frac{\partial \mathbf{H}_{(0, i)}^{T}}{\partial \mathbf{q}_{j}} \mathbf{H}_{(B, 0)}^{T} \mathbf{H}_{(I, B)}^{T}\right] \dot{z}_{s} \dot{q}_{j}+ \\
& \sum_{i=1}^{n_{s}} \sum_{s=1}^{6} \sum_{t=1}^{6} \frac{1}{2} \operatorname{tr}\left[\frac{\partial \mathbf{H}_{(I, B)}}{\partial \mathbf{z}_{s}} \mathbf{H}_{(B, 0)} \mathbf{H}_{(0, i)} \mathbf{J}_{i} \mathbf{H}_{(0, i)}^{T} \mathbf{H}_{(B, 0)}^{T} \frac{\partial \mathbf{H}_{(I, B)}^{T}}{\partial \mathbf{z}_{t}}\right] \dot{z}_{s} \dot{z}_{t}+ \\
& \sum_{i=1}^{n_{1}} \sum_{j=1}^{i} \sum_{k=1}^{i} \frac{1}{2} \operatorname{tr}\left[\mathbf{H}_{(I, B)} \mathbf{H}_{(B, 0)} \frac{\partial \mathbf{H}_{(0, i)}}{\partial \mathbf{q}_{j}} \mathbf{J}_{i} \frac{\partial \mathbf{H}_{(0, i)}^{T}}{\partial \mathbf{q}_{k}} \mathbf{H}_{(B, 0)}^{T} \mathbf{H}_{(I, B)}^{T}\right] \dot{q}_{j} \dot{q}_{k}
\end{aligned}
$$


The potential energy of link $i$ is written as

$$
P_{i}=-m_{i} \mathbf{g}_{i} \mathbf{H}_{(I, B)} \mathbf{H}_{(B, 0)} \mathbf{H}_{(0, i)} \mathbf{r}_{i}
$$

Refering to Figure 3.1 and following a similar derivation as for the links, the position of a point on the base in the base frame $F_{B}$ relative to and projected onto frame $F_{I}$ is given by

$$
\mathbf{b}_{I}=\mathbf{H}_{(I, B)} \mathbf{b}
$$

The velocity is then written as

$$
\mathbf{v}_{I B}=\frac{d\left(\mathbf{b}_{I}\right)}{d t}
$$

or

$$
\mathbf{v}_{I B}=\sum_{s=1}^{6} \frac{\partial \mathbf{H}_{(I, B)}}{\partial \mathbf{z}_{s}} \mathbf{b} \dot{z}_{s}
$$

Again, summing the kinetic energy of a particle of mass $d m$ at $\mathbf{b}$ on the base and integrating over the mass for the base we get the total base kinetic energy as

$$
K_{B}=\sum_{s=1}^{6} \sum_{t=1}^{6} \frac{1}{2} \operatorname{tr}\left[\frac{\partial \mathbf{H}_{(I, B)}}{\partial \mathbf{z}_{s}} \mathbf{J}_{B} \frac{\partial \mathbf{H}_{(I, B)}^{T}}{\partial \mathbf{z}_{t}}\right] \dot{z}_{s} \dot{z}_{t}
$$

where $\mathbf{J}_{B}$ is the base pseudo inertia matrix

$$
\mathbf{J}_{B}=\int_{b a s e} \mathbf{b b}^{T} d m
$$

If the base frame is at the centre of mass of the base we can also write the equivalent simpler form of the base K.E. as

$$
K_{B}=\frac{1}{2} m_{b} \dot{\mathbf{R}} \dot{\mathbf{R}}^{T}+\frac{1}{2} \mathbf{w} \mathbf{I}_{B} \mathbf{w}^{T}
$$

$=\mathrm{K} . \mathrm{E}$. (of the centre of mass) +K.E. (about centre of mass)

The potential energy of the base is given by

$$
P_{B}=-m_{b} \mathbf{g}_{i} \mathbf{H}_{(I, B)} \mathbf{b}
$$

where $\mathbf{g}^{T}=(g, 0,0,0)$. 
Thus the total kinetic energy of the manipulator-base system is

$$
\begin{aligned}
K_{S}=K_{L}+K_{B}= & \sum_{i=1}^{n} \sum_{s=1}^{6} \sum_{j=1}^{i} \operatorname{tr}\left[\frac{\partial \mathbf{H}_{(I, B)}}{\partial \mathbf{z}_{s}} \mathbf{H}_{(B, 0)} \mathbf{H}_{(0, i)} \mathbf{J}_{i} \frac{\partial \mathbf{H}_{(0, i)}^{T}}{\partial \mathbf{q}_{j}} \mathbf{H}_{(B, 0)}^{T} \mathbf{H}_{(I, B)}^{T}\right] \dot{z}_{s} \dot{q}_{j}+ \\
& \sum_{i=1}^{n} \sum_{s=1}^{6} \sum_{t=1}^{6} \frac{1}{2} \operatorname{tr}\left[\frac{\partial \mathbf{H}_{(I, B)}}{\partial \mathbf{z}_{s}} \mathbf{H}_{(B, 0)} \mathbf{H}_{(0, i)} \mathbf{J}_{i} \mathbf{H}_{(0, i)}^{T} \mathbf{H}_{(B, 0)}^{T} \frac{\partial \mathbf{H}_{(I, B)}^{T}}{\partial \mathbf{z}_{t}}\right] \dot{z}_{s} \dot{z}_{t}+ \\
& \sum_{i=1}^{n} \sum_{j=1}^{i} \sum_{k=1}^{i} \frac{1}{2} \operatorname{tr}\left[\mathbf{H}_{(I, B)} \mathbf{H}_{(B, 0)} \frac{\partial \mathbf{H}_{(0, i)}}{\partial \mathbf{q}_{j}} \mathbf{J}_{i} \frac{\partial \mathbf{H}_{(0, i)}^{T}}{\partial \mathbf{q}_{k}} \mathbf{H}_{(B, 0)}^{T} \mathbf{H}_{(I, B)}^{T}\right] \dot{q}_{j} \dot{q}_{k}+ \\
& \sum_{s=1}^{6} \sum_{t=1}^{6} \frac{1}{2} \operatorname{tr}\left[\frac{\partial \mathbf{H}_{(I, B)}}{\partial \mathbf{z}_{s}} \mathbf{J}_{B} \frac{\partial \mathbf{H}_{(I, B)}^{T}}{\partial \mathbf{z}_{t}}\right] \dot{z}_{s} \dot{z}_{t}
\end{aligned}
$$

and the total potential energy of the system is

$$
P_{S}=-\sum_{i=1}^{n} m_{i} \mathbf{g}_{i} \mathbf{H}_{(I, B)} \mathbf{H}_{(B, 0)} \mathbf{H}_{(0, i)} \mathbf{r}_{i}-m_{b} \mathbf{g}_{i} \mathbf{H}_{(I, B)} \mathbf{b}
$$

\subsubsection{Lagrange Equations}

To obtain the equations of motion we form the Lagrangian, $L=K-P$, and apply the Lagrange equation below

$$
\frac{d}{d t}\left(\frac{\partial \mathbf{L}}{\partial \dot{\mathbf{p}}_{i}}\right)-\frac{\partial \mathbf{L}}{\partial \mathbf{p}_{i}}=\tau_{i}
$$

where $p_{i}$ is the generalized coordinate and $\tau_{i}$ is the generalized force or torque at joint $i$. The Lagrangian becomes

$$
\begin{array}{r}
L=\sum_{i=1}^{n} \sum_{s=1}^{6} \sum_{j=1}^{i} \operatorname{tr}\left[\frac{\partial \mathbf{H}_{(I, B)}}{\partial \mathbf{z}_{s}} \mathbf{H}_{(B, 0)} \mathbf{H}_{(0, i)} \mathbf{J}_{i} \frac{\partial \mathbf{H}_{(0, i)}^{T}}{\partial \mathbf{q}_{j}} \mathbf{H}_{(B, 0)}^{T} \mathbf{H}_{(I, B)}^{T}\right] \dot{z}_{s} \dot{q}_{j}+ \\
\sum_{i=1}^{n} \sum_{s=1}^{6} \sum_{t=1}^{6} \frac{1}{2} \operatorname{tr}\left[\frac{\partial \mathbf{H}_{(I, B)}}{\partial \mathbf{z}_{s}} \mathbf{H}_{(B, 0)} \mathbf{H}_{(0, i)} \mathbf{J}_{i} \mathbf{H}_{(0, i)}^{T} \mathbf{H}_{(B, 0)}^{T} \frac{\partial \mathbf{H}_{(I, B)}^{T}}{\partial \mathbf{z}_{t}}\right] \dot{z}_{s} \dot{z}_{t}+ \\
\sum_{i=1}^{n} \sum_{j=1}^{i} \sum_{k=1}^{i} \frac{1}{2} \operatorname{tr}\left[\mathbf{H}_{(I, B)} \mathbf{H}_{(B, 0)} \frac{\partial \mathbf{H}_{(0, i)}}{\partial \mathbf{q}_{j}} \mathbf{J}_{i} \frac{\partial \mathbf{H}_{(0, i)}^{T}}{\partial \mathbf{q}_{k}} \mathbf{H}_{(B, 0)}^{T} \mathbf{H}_{(I, B)}^{T}\right] \dot{q}_{j} \dot{q}_{k}+ \\
\sum_{s=1}^{6} \sum_{t=1}^{6} \frac{1}{2} \operatorname{tr}\left[\frac{\partial \mathbf{H}_{(I, B)}}{\partial \mathbf{z}_{s}} \mathbf{J}_{B} \frac{\partial \mathbf{H}_{(I, B)}^{T}}{\partial \mathbf{z}_{t}}\right] \dot{z}_{s} \dot{z}_{t}+ \\
\sum_{i=1}^{n} m_{i} \mathbf{g}_{i} \mathbf{H}_{(I, B)} \mathbf{H}_{(B, 0)} \mathbf{H}_{(0, i)} \mathbf{r}_{i}+m_{b} \mathbf{g}_{i} \mathbf{H}_{(I, B)} \mathbf{b}
\end{array}
$$


Combining the base terms $\left(\dot{z}_{s} \dot{z}_{t}\right)$ we obtain

$$
\begin{array}{r}
L=\sum_{i=1}^{n} \sum_{s=1}^{6} \sum_{j=1}^{i} \operatorname{tr}\left[\frac{\partial \mathbf{H}_{(I, B)}}{\partial \mathbf{z}_{s}} \mathbf{H}_{(B, 0)} \mathbf{H}_{(0, i)} \mathbf{J}_{i} \frac{\partial \mathbf{H}_{(0, i)}^{T}}{\partial \mathbf{q}_{j}} \mathbf{H}_{(B, 0)}^{T} \mathbf{H}_{(I, B)}^{T}\right] \dot{z}_{s} \dot{q}_{j}+ \\
\sum_{i=1}^{n} \sum_{s=1}^{6} \sum_{t=1}^{6} \frac{1}{2} t r\left[\frac{\partial \mathbf{H}_{(I, B)}}{\partial \mathbf{z}_{s}} \mathbf{H}_{(B, 0)} \mathbf{H}_{(0, i)} \mathbf{J}_{i} \mathbf{H}_{(0, i)}^{T} \mathbf{H}_{(B, 0)}^{T} \frac{\partial \mathbf{H}_{(I, B)}^{T}}{\partial \mathbf{z}_{t}}+\frac{\partial \mathbf{H}_{(I, B)}}{\partial \mathbf{z}_{s}} \mathbf{J}_{B} \frac{\partial \mathbf{H}_{(I, B)}^{T}}{\partial \mathbf{z}_{t}}\right] \dot{z}_{s} \dot{z}_{t}+ \\
\sum_{i=1}^{n} \sum_{j=1}^{i} \sum_{k=1}^{i} \frac{1}{2} \operatorname{tr}\left[\mathbf{H}_{(I, B)} \mathbf{H}_{(B, 0)} \frac{\partial \mathbf{H}_{(0, i)}}{\partial \mathbf{q}_{j}} \mathbf{J}_{i} \frac{\partial \mathbf{H}_{(0, i)}^{T}}{\partial \mathbf{q}_{k}} \mathbf{H}_{(B, 0)}^{T} \mathbf{H}_{(I, B)}^{T}\right] \dot{q}_{j} \dot{q}_{k}+ \\
\sum_{i=1}^{n} m_{i} \mathbf{g}_{i} \mathbf{H}_{(I, B)} \mathbf{H}_{(B, 0)} \mathbf{H}_{(0, i)} \mathbf{r}_{i}+m_{b} \mathbf{g}_{i} \mathbf{H}_{(I, B)} \mathbf{b}
\end{array}
$$

For the link dynamic equations, we will let the manipulator link generalized coordinates be represented as $q_{p}$. Taking the derivative of $\mathbf{L}$ with repect to $\dot{q}_{p}$ we obtain

$$
\begin{aligned}
\frac{\partial \mathbf{L}}{\partial \dot{\mathbf{p}}_{i}}= & \sum_{i=p}^{n} \sum_{s=1}^{6} \operatorname{tr}\left[\frac{\partial \mathbf{H}_{(I, B)}}{\partial \mathbf{z}_{s}} \mathbf{H}_{(B, 0)} \mathbf{H}_{(0, i)} \mathbf{J}_{i} \frac{\partial \mathbf{H}_{(0, i)}^{T}}{\partial \mathrm{q}_{p}} \mathbf{H}_{(B, 0)}^{T} \mathbf{H}_{(I, B)}^{T}\right] \dot{z}_{s}+ \\
& \sum_{i=p}^{n} \sum_{k=1}^{i} \operatorname{tr}\left[\mathbf{H}_{(I, B)} \mathbf{H}_{(B, 0)} \frac{\partial \mathbf{H}_{(0, i)}}{\partial \mathbf{q}_{p}} \mathbf{J}_{i} \frac{\partial \mathbf{H}_{(0, i)}^{T}}{\partial \mathbf{q}_{k}} \mathbf{H}_{(B, 0)}^{T} \mathbf{H}_{(I, B)}^{T}\right] \dot{q}_{k}
\end{aligned}
$$

We can combine terms 2 and 3 because of similar indices and also because trace $(A)=\operatorname{trace}\left(A^{T}\right)$. We also know that for $p>i$,

$$
\frac{\partial \mathbf{H}_{(0, i)}}{\partial \mathbf{q}_{p}}=0
$$

Therefore

$$
\begin{aligned}
\frac{\partial \mathbf{L}}{\partial \dot{\mathbf{p}}_{i}}= & \sum_{i=p}^{n} \sum_{s=1}^{6} \operatorname{tr}\left[\frac{\partial \mathbf{H}_{(I, B)}}{\partial \mathbf{z}_{s}} \mathbf{H}_{(B, 0)} \mathbf{H}_{(0, i)} \mathbf{J}_{i} \frac{\partial \mathbf{H}_{(0, i)}^{T}}{\partial \mathbf{q}_{p}} \mathbf{H}_{(B, 0)}^{T} \mathbf{H}_{(I, B)}^{T}\right] \dot{z}_{s}+ \\
& \sum_{i=p}^{n} \sum_{k=1}^{i} \operatorname{tr}\left[\mathbf{H}_{(I, B)} \mathbf{H}_{(B, 0)} \frac{\partial \mathbf{H}_{(0, i)}}{\partial \mathbf{q}_{p}} \mathbf{J}_{i} \frac{\partial \mathbf{H}_{(0, i)}^{T}}{\partial \mathbf{q}_{k}} \mathbf{H}_{(B, 0)}^{T} \mathbf{H}_{(I, B)}^{T}\right] \dot{q}_{k}
\end{aligned}
$$

Taking the time derivative of the above we obtain

$$
\begin{array}{r}
\frac{d}{d t}\left(\frac{\partial \mathbf{L}}{\partial \dot{\mathbf{p}}_{i}}\right)=\sum_{i=p}^{n} \sum_{s=1}^{6} \operatorname{tr}\left[\frac{\partial \mathbf{H}_{(I, B)}}{\partial \mathbf{z}_{s}} \mathbf{H}_{(B, 0)} \mathbf{H}_{(0, i)} \mathbf{J}_{i} \frac{\partial \mathbf{H}_{(0, i)}^{T}}{\partial \mathbf{q}_{p}} \mathbf{H}_{(B, 0)}^{T} \mathbf{H}_{(I, B)}^{T}\right] \ddot{z}_{s}+ \\
\sum_{i=p}^{n} \sum_{s=1}^{6} \sum_{t=1}^{6} \operatorname{tr}\left[\frac{\partial^{2} \mathbf{H}_{(I, B)}}{\partial \mathbf{z}_{t} \partial \mathbf{z}_{s}} \mathbf{H}_{(B, 0)} \mathbf{H}_{(0, i)} \mathbf{J}_{i} \frac{\partial \mathbf{H}_{(0, i)}^{T}}{\partial \mathbf{q}_{p}} \mathbf{H}_{(B, 0)}^{T} \mathbf{H}_{(I, B)}^{T}\right] \dot{z}_{s} \dot{z}_{t}+
\end{array}
$$




$$
\begin{gathered}
\sum_{i=p}^{n} \sum_{s=1}^{6} \sum_{m=1}^{i} \operatorname{tr}\left[\frac{\partial \mathbf{H}_{(I, B)}}{\partial \mathbf{z}_{s}} \mathbf{H}_{(B, 0)} \frac{\partial \mathbf{H}_{(0, i)}}{\partial \mathbf{q}_{m}} \mathbf{J}_{i} \frac{\partial \mathbf{H}_{(0, i)}^{T}}{\partial \mathbf{q}_{p}} \mathbf{H}_{(B, 0)}^{T} \mathbf{H}_{(I, B)}^{T}\right] \dot{z}_{s} \dot{q}_{m}+ \\
\sum_{i=p}^{n} \sum_{s=1}^{6} \sum_{m=1}^{i} \operatorname{tr}\left[\frac{\partial \mathbf{H}_{(I, B)}}{\partial \mathbf{z}_{s}} \mathbf{H}_{(B, 0)} \mathbf{H}_{(0, i)} \mathbf{J}_{i} \frac{\partial^{2} \mathbf{H}_{(0, i)}^{T}}{\partial \mathbf{q}_{m} \partial \mathbf{q}_{p}} \mathbf{H}_{(B, 0)}^{T} \mathbf{H}_{(I, B)}^{T}\right] \dot{z}_{s} \dot{q}_{m}+ \\
\sum_{i=p}^{n} \sum_{s=1}^{6} \sum_{t=1}^{6} \operatorname{tr}\left[\frac{\partial \mathbf{H}_{(I, B)}}{\partial \mathbf{z}_{s}} \mathbf{H}_{(B, 0)} \mathbf{H}_{(0, i)} \mathbf{J}_{i} \frac{\partial \mathbf{H}_{(0, i)}^{T}}{\partial \mathbf{q}_{p}} \mathbf{H}_{(B, 0)}^{T} \frac{\partial \mathbf{H}_{(I, B)}^{T}}{\partial \mathbf{z}_{t}}\right] \dot{z}_{s} \dot{z}_{t}+ \\
\sum_{i=p}^{n} \sum_{k=1}^{n} \sum_{s=1}^{i} \sum_{k=1}^{i} \operatorname{tr}\left[\frac{\partial \mathbf{H}_{(I, B)}}{\partial \mathbf{z}_{s}} \mathbf{H}_{(B, 0)} \frac{\partial \mathbf{H}_{(0, i)}}{\partial \mathbf{q}_{p}} \mathbf{J}_{i} \frac{\partial \mathbf{H}_{(0, i)}^{T}}{\partial \mathbf{q}_{k}} \mathbf{H}_{(B, 0)}^{T} \mathbf{H}_{(B, 0)}^{T} \frac{\partial \mathbf{H}_{(0, i)}}{\partial \mathbf{q}_{p}} \mathbf{J}_{i} \frac{\partial \mathbf{H}_{(0, i)}^{T}}{\partial \mathbf{q}_{k}} \mathbf{H}_{(B, 0)}^{T} \mathbf{H}_{(I, B)}^{T}\right] \ddot{q}_{k}+ \\
\sum_{i=p}^{n} \sum_{k=1}^{i} \sum_{m=1}^{i} \operatorname{tr}\left[\mathbf{z}_{(I, B)} \mathbf{H}_{(B, 0)} \frac{\partial^{2} \mathbf{H}_{(0, i)}}{\partial \mathbf{q}_{m} \partial \mathbf{q}_{p}} \mathbf{J}_{i} \frac{\partial \mathbf{H}_{(0, i)}^{T}}{\partial \mathbf{q}_{k}} \mathbf{H}_{(B, 0)}^{T} \mathbf{H}_{(I, B)}^{T}\right] \dot{q}_{k} \dot{q}_{m}+ \\
\sum_{i=p}^{n} \sum_{k=1}^{i} \sum_{m=1}^{i} t r\left[\mathbf{H}_{(I, B)} \mathbf{H}_{(B, 0)} \frac{\partial \mathbf{H}_{(0, i)}}{\partial \mathbf{q}_{p}} \mathbf{J}_{i} \frac{\partial^{2} \mathbf{H}_{(0, i)}^{T}}{\partial \mathbf{q}_{m} \partial \mathbf{q}_{k}} \mathbf{H}_{(B, 0)}^{T} \mathbf{H}_{(I, B)}^{T}\right] \dot{q}_{k} \dot{q}_{m}+ \\
\sum_{i=p}^{n} \sum_{k=1}^{i} \sum_{s=1}^{6} t r\left[\mathbf{H}_{(I, B)} \mathbf{H}_{(B, 0)} \frac{\partial \mathbf{H}_{(0, i)}}{\partial \mathbf{q}_{p}} \mathbf{J}_{i} \frac{\partial \mathbf{H}_{(0, i)}^{T}}{\partial \mathbf{q}_{k}} \mathbf{H}_{(B, 0)}^{T} \mathbf{H}_{(I, B)}^{T}\right] \dot{q}_{k} \dot{z}_{s}
\end{gathered}
$$

For the second term in the Lagrange equation for the links we obtain

$$
\begin{aligned}
& \frac{\partial \mathbf{L}}{\partial \mathbf{p}_{i}}= \sum_{i=1}^{n} \sum_{s=1}^{6} \sum_{j=1}^{i} \operatorname{tr}\left[\frac{\partial \mathbf{H}_{(I, B)}}{\partial \mathbf{z}_{s}} \mathbf{H}_{(B, 0)} \frac{\partial \mathbf{H}_{(0, i)}}{\partial \mathbf{q}_{p}} \mathbf{J}_{i} \frac{\partial \mathbf{H}_{(0, i)}^{T}}{\partial \mathbf{q}_{j}} \mathbf{H}_{(B, 0)}^{T} \mathbf{H}_{(I, B)}^{T}\right] \dot{z}_{s} \dot{q}_{j}+ \\
& \sum_{i=1}^{n} \sum_{s=1}^{6} \sum_{j=1}^{i} \operatorname{tr}\left[\frac{\partial \mathbf{H}_{(I, B)}}{\partial \mathbf{z}_{s}} \mathbf{H}_{(B, 0)} \mathbf{H}_{(0, i)} \mathbf{J}_{i} \frac{\partial^{2} \mathbf{H}_{(0, i)}^{T}}{\partial \mathbf{q}_{p} \partial \mathbf{q}_{j}} \mathbf{H}_{(B, 0)}^{T} \mathbf{H}_{(I, B)}^{T}\right] \dot{z}_{s} \dot{q}_{j}+ \\
& \sum_{i=1}^{n} \sum_{s=1}^{6} \sum_{t=1}^{6} \frac{1}{2} \operatorname{tr}\left[\frac{\partial \mathbf{H}_{(I, B)}}{\partial \mathbf{z}_{s}} \mathbf{H}_{(B, 0)} \frac{\partial \mathbf{H}_{(0, i)}}{\partial \mathbf{q}_{p}} \mathbf{J}_{i} \mathbf{H}_{(0, i)}^{T} \mathbf{H}_{(B, 0)}^{T} \frac{\partial \mathbf{H}_{(I, B)}^{T}}{\partial \mathbf{z}_{t}}\right] \dot{z}_{s} \dot{z}_{t}+ \\
& \sum_{i=1}^{n} \sum_{s=1}^{6} \sum_{t=1}^{6} \frac{1}{2} \operatorname{tr}\left[\frac{\partial \mathbf{H}_{(I, B)}}{\partial \mathbf{z}_{s}} \mathbf{H}_{(B, 0)} \mathbf{H}_{(0, i)} \mathbf{J}_{i} \frac{\partial \mathbf{H}_{(0, i)}^{T}}{\partial \mathbf{q}_{p}} \mathbf{H}_{(B, 0)}^{T} \frac{\partial \mathbf{H}_{(I, B)}^{T}}{\partial \mathbf{z}_{t}}\right] \dot{z}_{s} \dot{z}_{t}+ \\
& \sum_{i=1}^{n} \sum_{j=1}^{i} \sum_{k=1}^{i} \frac{1}{2} \operatorname{tr}\left[\mathbf{H}_{(I, B)} \mathbf{H}_{(B, 0)} \frac{\partial^{2} \mathbf{H}_{(0, i)}}{\partial \mathbf{q}_{j} \partial \mathbf{q}_{p}} \mathbf{J}_{i} \frac{\partial \mathbf{H}_{(0, i)}^{T}}{\partial \mathbf{q}_{k}} \mathbf{H}_{(B, 0)}^{T} \mathbf{H}_{(I, B)}^{T}\right] \dot{q}_{j} \dot{q}_{k}+ \\
& \sum_{i=1}^{n} \sum_{j=1}^{i} \sum_{k=1}^{i} \frac{1}{2} \operatorname{tr}\left[\mathbf{H}_{(I, B)} \mathbf{H}_{(B, 0)} \frac{\partial \mathbf{H}_{(0, i)}}{\partial \mathbf{q}_{j}} \mathbf{J}_{i} \frac{\partial^{2} \mathbf{H}_{(0, i)}^{T}}{\partial \mathbf{q}_{k} \partial \mathbf{q}_{p}} \mathbf{H}_{(B, 0)}^{T} \mathbf{H}_{(I, B)}^{T}\right] \dot{q}_{j} \dot{q}_{k}+ \\
& \sum_{i=1}^{n} m_{i} \mathbf{g}_{i} \mathbf{H}_{(I, B)} \mathbf{H}_{(B, 0)} \frac{\partial \mathbf{H}_{(I, 0)}}{\partial \mathbf{q}_{p}} \mathbf{r}_{i}
\end{aligned}
$$

Forming the Lagrange equation with the last two equations, after many cancellations and index 
substitutions we obtain the dynamic equations for the manipulator links. With the generalized coordinate for the link denoted as $q_{i}$ and the generalized force or torque as $\tau_{i}$ the equation of motion becomes

$$
\begin{aligned}
& \sum_{i=p}^{n} \sum_{s=1}^{6} \operatorname{tr}\left[\frac{\partial \mathbf{H}_{(I, B)}}{\partial \mathbf{z}_{s}} \mathbf{H}_{(B, 0)} \mathbf{H}_{(0, i)} \mathbf{J}_{i} \frac{\partial \mathbf{H}_{(0, i)}^{T}}{\partial \mathbf{q}_{p}} \mathbf{H}_{(B, 0)}^{T} \mathbf{H}_{(I, B)}^{T}\right] \ddot{z}_{s}+ \\
& \sum_{i=p}^{n} \sum_{k=1}^{i} \operatorname{tr}\left[\mathbf{H}_{(I, B)} \mathbf{H}_{(B, 0)} \frac{\partial \mathbf{H}_{(0, i)}}{\partial \mathrm{q}_{p}} \mathbf{J}_{i} \frac{\partial \mathbf{H}_{(0, i)}^{T}}{\partial \mathrm{q}_{k}} \mathbf{H}_{(B, 0)}^{T} \mathbf{H}_{(I, B)}^{T}\right] \ddot{q}_{k}+ \\
& \sum_{i=p}^{n} \sum_{s=1}^{6} \sum_{t=1}^{6} t r\left[\frac{\partial^{2} \mathbf{H}_{(I, B)}}{\partial \mathbf{z}_{t} \partial \mathbf{z}_{s}} \mathbf{H}_{(B, 0)} \mathbf{H}_{(0, i)} \mathbf{J}_{i} \frac{\partial \mathbf{H}_{(0, i)}^{T}}{\partial \mathbf{q}_{p}} \mathbf{H}_{(B, 0)}^{T} \mathbf{H}_{(I, B)}^{T}\right] \dot{z}_{s} \dot{z}_{t}+ \\
& \sum_{i=p}^{n} \sum_{k=1}^{i} \sum_{m=1}^{i} t r\left[\mathbf{H}_{(I, B)} \mathbf{H}_{(B, 0)} \frac{\partial \mathbf{H}_{(0, i)}}{\partial \mathbf{q}_{p}} \mathbf{J}_{i} \frac{\partial^{2} \mathbf{H}_{(0, i)}^{T}}{\partial \mathbf{q}_{m} \partial \mathbf{q}_{k}} \mathbf{H}_{(B, 0)}^{T} \mathbf{H}_{(I, B)}^{T}\right] \dot{q}_{k} \dot{q}_{m}+ \\
& \sum_{i=p}^{n_{n}} \sum_{s=1}^{6} \sum_{m=1}^{i}(2) \operatorname{tr}\left[\frac{\partial \mathbf{H}_{(I, B)}}{\partial \mathbf{z}_{s}} \mathbf{H}_{(B, 0)} \frac{\partial \mathbf{H}_{(0, i)}}{\partial \mathbf{q}_{m}} \mathbf{J}_{i} \frac{\partial \mathbf{H}_{(0, i)}^{T}}{\partial \mathbf{q}_{p}} \mathbf{H}_{(B, 0)}^{T} \mathbf{H}_{(I, B)}^{T}\right] \dot{z}_{s} \dot{q}_{m}+ \\
& \sum_{i=1}^{n} m_{i} \mathbf{g}_{i} \mathbf{H}_{(I, B)} \mathbf{H}_{(B, 0)} \frac{\partial \mathbf{H}_{(I, 0)}}{\partial \mathbf{q}_{p}} \mathbf{r}_{i}=\tau_{p}
\end{aligned}
$$

- $p=1$ gives the equation for link 1 in $q_{1}$

- $p=x$ gives the $\mathrm{n}$ th equation for link $x$ in $q_{x}$

To obtain the dynamic equations of the base, we apply the Lagrange equation to the Lagrangian except this time $z_{w}$ will be the generalized base coordinate

$$
\frac{d}{d t}\left(\frac{\partial \mathbf{L}}{\partial \dot{\mathbf{z}}_{w}}\right)-\frac{\partial \mathbf{L}}{\partial \mathbf{z}_{w}}=0
$$

The zero on the right hand side of the equation is because there are no external forces or torques acting on the base such as thruster control jets or momentum gyros.

The derivation of the dynamic equation for the base follows similar algebra as for the links, and after simplifications becomes

$$
\sum_{i=1}^{n} \sum_{t=1}^{6} \operatorname{tr}\left[\frac{\partial \mathbf{H}_{(I, B)}}{\partial \mathbf{z}_{w}} \mathbf{H}_{(B, 0)} \mathbf{H}_{(0, i)} \mathbf{J}_{i} \mathbf{H}_{(0, i)}^{T} \mathbf{H}_{(B, 0)}^{T} \frac{\partial \mathbf{H}_{(I, B)}^{T}}{\partial \mathbf{z}_{t}}+\frac{\partial \mathbf{H}_{(I, B)}}{\partial \mathbf{z}_{w}} \mathbf{J}_{B} \frac{\partial \mathbf{H}_{(I, B)}^{T}}{\partial \mathbf{z}_{t}}\right] \ddot{z}_{t}+
$$




$$
\begin{aligned}
& \sum_{i=1}^{n} \sum_{j=1}^{i} \operatorname{tr}\left[\frac{\partial \mathbf{H}_{(I, B)}}{\partial \mathbf{z}_{w}} \mathbf{H}_{(B, 0)} \mathbf{H}_{(0, i)} \mathbf{J}_{i} \frac{\partial \mathbf{H}_{(0, i)}^{T}}{\partial \mathbf{q}_{j}} \mathbf{H}_{(B, 0)}^{T} \mathbf{H}_{(I, B)}^{T}\right] \ddot{q}_{j}+ \\
& \sum_{i=1}^{n} \sum_{t=1}^{6} \sum_{s=1}^{6} \operatorname{tr}\left[\frac{\partial \mathbf{H}_{(I, B)}}{\partial \mathbf{z}_{w}} \mathbf{H}_{(B, 0)} \mathbf{H}_{(0, i)} \mathbf{J}_{i} \mathbf{H}_{(0, i)}^{T} \mathbf{H}_{(B, 0)}^{T} \frac{\partial^{2} \mathbf{H}_{(I, B)}^{T}}{\partial \mathbf{z}_{s} \partial \mathbf{z}_{t}}+\frac{\partial \mathbf{H}_{(I, B)}}{\partial \mathbf{z}_{w}} \mathbf{J}_{B} \frac{\partial^{2} \mathbf{H}_{(I, B)}^{T}}{\partial \mathbf{z}_{s} \partial \mathbf{z}_{t}}\right] \dot{z}_{s} \dot{z}_{t}+ \\
& \sum_{i=1}^{n} \sum_{j=1}^{i} \sum_{m=1}^{i} t r\left[\frac{\partial \mathbf{H}_{(I, B)}}{\partial \mathbf{z}_{w}} \mathbf{H}_{(B, 0)} \mathbf{H}_{(0, i)} \mathbf{J}_{i} \frac{\partial^{2} \mathbf{H}_{(0, i)}^{T}}{\partial \mathbf{q}_{m} \partial \mathbf{q}_{j}} \mathbf{H}_{(B, 0)}^{T} \mathbf{H}_{(I, B)}^{T}\right] \dot{q}_{j} \dot{q}_{m}+ \\
& \sum_{i=1}^{n} \sum_{j=1}^{n} \sum_{s=1}^{i}(2) t r\left[\frac{\partial \mathbf{H}_{(I, B)}}{\partial \mathbf{z}_{w}} \mathbf{H}_{(B, 0)} \mathbf{H}_{(0, i)} \mathbf{J}_{i} \frac{\partial \mathbf{H}_{(0, i)}^{T}}{\partial \mathbf{q}_{j}} \mathbf{H}_{(B, 0)}^{T} \mathbf{H}_{(I, B)}^{T} z s\right] \dot{q}_{j} \dot{z}_{s}+m_{b} \mathbf{g}_{i} \frac{\partial \mathbf{H}_{(I, B)}}{\partial \mathbf{z}_{w}} \mathbf{b}=0 \\
& \text { • } w=1 \text { gives the } 1 \text { st equation for } z_{w} \\
& \text { • } w=6 \text { gives the } 6 \text { th equation for } z_{6}
\end{aligned}
$$

The above manipulator dynamic equations have been developed in three dimensions for an $n$ link manipulator on a 6 degree of freedom base, assuming there is gravity acting on the system. For a space application, gravity would be set to zero. These dynamic equations can also be written in matrix form as

$$
\mathbf{M}(q) \ddot{q}+\mathbf{V}(q, \dot{q})+\mathbf{G}(q)=\tau
$$

where $\mathbf{M}$ is the inertia matrix, $\mathbf{V}$ is the Coriolis-centripetal matrix, $\mathbf{G}$ is the gravity matrix, $\tau$ is the generalized force (torque) vector, and $q$ is the generalized coordinate. If a disturbance term, $\mathbf{F}(\dot{q})$, is added to the above equation the manipulator dynamic equation becomes

$$
\mathbf{M}(q) \ddot{q}+\mathbf{V}(q, \dot{q})+\mathbf{F}(\dot{q})+\mathbf{G}(q)=\tau
$$

or more compactly

$$
\mathbf{M}(q) \ddot{q}+\mathbf{N}(q, \dot{q})=\tau
$$

where $\mathbf{N}(\mathbf{q}, \dot{\mathbf{q}})$ represents all the non-linear terms. 


\subsubsection{Equation Expansion using MAPLE}

The simulations and algorithms developed in this thesis were done in 2 dimensions. For the 2 dimensional case, the base is capable of moving in 2 translational directions as well as one rotational direction. Therefore the base possesses 3 d.o.f. and has 3 generalized coordinates. A 2 link revolute manipulator is used and has 2 d.o.f., bringing the total to 5 d.o.f. for the planar case. The homogeneous matrices for the links will reflect whether the links are revolute or prismatic. This will result in 5 non-linear coupled differential equations containing 5 generalized coordinates. To obtain equations that are ready for simulation, these dynamic equations of motion must be fully expanded and then converted into state-space form. Expanding the link and base equations by hand, (equations 3.34 and 3.36 respectively), is a large error-prone undertaking. Each equation, after expansion, of the double and triple summations results in up to 54 terms that involve the multiplication of 5 to 7 homogeneous matrices. The trace of the resultant matrix terms must then be determined. The results are equations hundreds of terms long that are then to be used for simulations. After carrying out this procedure by hand and finding many errors, the symbolic algebra program Maple was used to do the equation expansion and simplification. Maple was used because of the availability of the program at the place where the author worked for part of his thesis, namely, International Submarine Engineering Ltd.

Equations 3.34 and 3.36 were programmed in Maple code and the program expanded the summations and performed the traces. The resulting equations are presented in Appendix A. First the homogeneous transformation and pseudo inertia matrices had to be defined. Some basic trigonometric simplification is performed by Maple automatically. Maple also has special functions such as the combine and collect functions for more advanced simplification. Maple's cost function gives the number of floating point operations (flops) for an equation and so some experimentation was done to optimize the equations (minimize computational cost). Once the dynamic equations of motion were developed the system of equations had to be converted to state space form for use in Matlab. State space form of the system dynamic equations is 
achieved by rearranging the equations so that they are in the form of first order differential equations. Taking the general system equation described in the previous section

$$
\mathrm{M}(q) \ddot{q}+\mathbf{N}(q, \dot{q})=\tau
$$

and rewriting in the nonlinear state-space form, we obtain

$$
\dot{\mathbf{Q}}=f(\mathbf{Q}, u, t)
$$

where $\mathbf{Q}$ is the state vector, $u(t)$ is the control input, and $t$ is the time. The time response simulator algorithm used in Matlab uses a Runge-Kutta integration routine that requires the computation of the state derivative given the current state. The 5 dynamic equations as shown in the abbreviated form are

$$
\begin{aligned}
& A_{1} \ddot{z}_{1}+A_{2} \ddot{z}_{2}+A_{3} \ddot{z}_{3}+A_{4} \ddot{q}_{1}+A_{5} \ddot{q}_{2}+A_{6}= 0 \\
& B_{1} \ddot{z}_{1}+B_{2} \ddot{z}_{2}+B_{3} \ddot{z}_{3}+B_{4} \ddot{q}_{1}+B_{5} \ddot{q}_{2}+B_{6}= 0 \\
& C_{1} \ddot{z}_{1}+C_{2} \ddot{z}_{2}+C_{3} \ddot{z}_{3}+C_{4} \ddot{q}_{1}+C_{5} \ddot{q}_{2}+C_{6}= 0 \\
& D_{1} \ddot{z}_{1}+D_{2} \ddot{z}_{2}+D_{3} \ddot{z}_{3}+D_{4} \ddot{q}_{1}+D_{5} \ddot{q}_{2}+D_{6}=\tau_{1} \\
& E_{1} \ddot{z}_{1}+E_{2} \ddot{z_{2}}+E_{3} \ddot{z}_{3}+E_{4} \ddot{q}_{1}+E_{5} \ddot{q}_{2}+E_{6}=\tau_{2}
\end{aligned}
$$

These equations are then put into matrix form with the Maple command genmatrix where column 6 of the above matrix are the Coriolis, centripetal, and torque terms. The resulting equation is factored and written as

$$
\left(\begin{array}{ccccc}
A_{1} & A_{2} & A_{3} & A_{4} & A_{5} \\
B_{1} & B_{2} & B_{3} & B_{4} & B_{5} \\
C_{1} & C_{2} & C_{3} & C_{4} & C_{5} \\
D_{1} & D_{2} & D_{3} & D_{4} & D_{5} \\
E_{1} & E_{2} & E_{3} & E_{4} & E_{5}
\end{array}\right)\left(\begin{array}{c}
\ddot{z}_{1} \\
\ddot{z}_{2} \\
\ddot{z}_{3} \\
\ddot{q}_{1} \\
\ddot{q}_{2}
\end{array}\right)=\left(\begin{array}{c}
-A_{6} \\
-B_{6} \\
-C_{6} \\
\tau_{1}-D_{6} \\
\tau_{2}-E_{6}
\end{array}\right)
$$


or

$$
\left(\begin{array}{c}
\ddot{z}_{1} \\
\ddot{z}_{2} \\
\ddot{z}_{3} \\
\ddot{q}_{1} \\
\ddot{q}_{2}
\end{array}\right)=\left(\begin{array}{lllll}
A_{1} & A_{2} & A_{3} & A_{4} & A_{5} \\
B_{1} & B_{2} & B_{3} & B_{4} & B_{5} \\
C_{1} & C_{2} & C_{3} & C_{4} & C_{5} \\
D_{1} & D_{2} & D_{3} & D_{4} & D_{5} \\
E_{1} & E_{2} & E_{3} & E_{4} & E_{5}
\end{array}\right)\left(\begin{array}{c}
-A_{6} \\
-B_{6} \\
-C_{6} \\
\tau_{1}-D_{6} \\
\tau_{2}-E_{6}
\end{array}\right)
$$

Rewriting the equation in state space form after defining the state vector,

$$
Q=\left(\begin{array}{llllllllll}
Z_{1} & \dot{Z}_{1} & Z_{2} & \dot{Z}_{2} & Z_{3} & \dot{Z}_{3} & Q_{1} & \dot{Q}_{1} & Q_{2} & \dot{Q}_{2}
\end{array}\right)
$$

where

$$
Z_{1}=\dot{z}_{1}
$$

and

$$
\dot{Z}_{1}=\ddot{z}_{1}
$$

The Maple command C [eqn] converts the Maple output into C code which is identical to Matlab code (except for the line breaking). The result is the dynamic equations of motion are in state space form, ready to be used.

\subsubsection{Equation Verification using MAPLE}

To verify that the resulting manipulator dynamic equations are correct, two methods are used. The first is to set all of the base variables and parameters equal to zero in the final dynamic equations and then confirm whether the remaining equations describe a two link revolute manipulator. The remaining equations did describe a two link manipulator and therefore the corresponding segment was shown to be correct. The link variables were also set to zero and the remaining equations correctly described a base (solid) moving in two dimensions. The second method utilized the conservation of momentum and angular momentum. As applied to this case, these principles state that in the absence of external forces or torques, after a 
manipulator-base movement, the centre of mass of the system should not change (invariant) and the linear and angular momentum should be the same as at the start of a movement. To check, a plot of the centre of mass of the system during the movement is viewed. Prior to using Maple (with the equations not quite correct) the results showed that the centre of mass was moving (and not stopping at the end of a movement) and hence there was an error in the equations. After using Maple for the equation expansion, the results showed that the centre of mass remained stationary throughout the movement and after it ended. Plots of the centre of mass position and velocity for a correct and incorrect set of equations can be seen in Figure 3.2 . The position and velocity of the correct set of equations are seen as a circle at the centre of the plots (stationary). The other trajectory shows movement and was obtained by changing a couple of signs in the Coriolis terms for the first two dynamic equations (base displacement equations). Clearly, using the conservation of momentum and angular momentum equations to check equation validity is very convenient and beneficial.
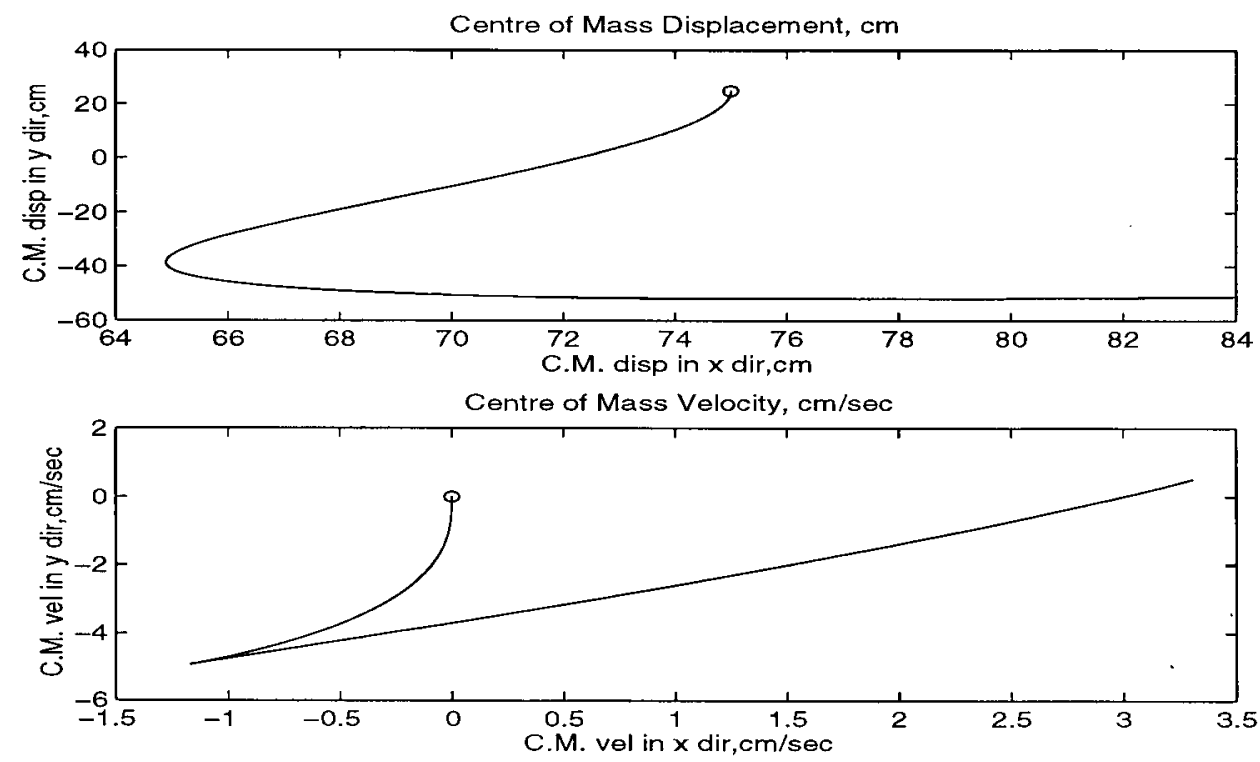

Figure 3.2: Centre of Mass Moving - Wrong Equations 
Another way the equations are checked for correctness is by observation. For example, the equations for link 2 should have the appropriate link 2 terms as well as the Coriolis and centripetal terms from link 1 and the base. This type of checking was made simpler since the Lagrange method was used for the equation derivation as opposed to Newton-Euler or quaternion methods. The drawback of using the Lagrange method as opposed to the NewtonEuler is that the resulting equations are much more computationally intensive. A discussion of the trade-off between equation transparency (ability to understand a terms meaning) and efficiency can be found in any robotics textbook in the dynamics section, such as in [Fu 87], page 103 .

\subsection{Joint Trajectories}

To move a robotic end-effector from point A to point B in Cartesian space, each robot joint degree of freedom must follow a prescribed trajectory in joint space. There are many paths or curves that can be used to get from one endpoint to another, and these are dependent on what type of path the end effector is to follow. The simplest type of robot motion is point to point motion, where the robot is commanded to go from an initial configuration to a final configuration without regard to the intermediate path followed by the end-effector. This type of motion is suitable if the workspace is clear of obstacles. If obstacles are present the end-effector would travel from one intermediate point to another, called via points, to avoid collisions. In this work we are not concerned with obstacles and hence can use smooth interpolating polynomials for joint space trajectory generation which satisfy the given boundary conditions. Even so there are many types of interpolating polynomials to choose from depending on whether the fastest time for a movement is desired, whether a constant velocity segment is required, or whether just constant acceleration is desired. It is also possible to piece different types together. A number of polynomial trajectories were evaluated, and a simple type, a quintic polynomial trajectory was chosen. A polynomial of this order is the minimum required to satisfy the endpoint boundary 
conditions that match continuously the joint angle, angular velocity, and angular accelerations. A discussion of polynomial trajectories can be found in [An 88]. The quintic trajectory is of the form

$$
c_{i}^{d}=a_{0}+a_{1} t+a_{2} t^{2}+a_{3} t^{3}+a_{4} t^{4}+a_{5} t^{5}
$$

The desired velocity trajectory is of the form

$$
\dot{c}_{i}^{d}=a_{1}+2 a_{2} t+3 a_{3} t^{2}+4 a_{4} t^{3}+5 a_{5} t^{4}
$$

and the desired acceleration trajectory is of the form

$$
\ddot{c}_{i}^{d .}=2 a_{2}+6 a_{3} t+12 a_{4} t^{2}+20 a_{5} t^{3}
$$

These equations satisfy the boundary conditions

$$
\begin{aligned}
& c_{i}^{d}\left(t_{0}\right)=c_{0} ; \quad c_{i}^{d}\left(t_{f}\right)=c_{f} \\
& \dot{c}_{i}^{d}\left(t_{0}\right)=\dot{c}_{0} ; \quad \dot{c}_{i}^{d}\left(t_{f}\right)=\dot{c}_{f} \\
& \ddot{c}_{i}^{d}\left(t_{0}\right)=\ddot{c}_{0} ; \quad \ddot{c}_{i}^{d}\left(t_{f}\right)=\ddot{c}_{f}
\end{aligned}
$$

These trajectories can be seen in Figure 3.3. Further discussion of joint trajectory updating is discussed in Section 4.4.

\subsection{Joint Servo Control}

A problem in robot control is to find the appropriate forces or torques to servo all the joints of the manipulator in order to track a desired time-based trajectory as closely as possible. Of the many types of robot control schemes, most can be considered to be special cases of the class of computed torque controllers. The most simple type of robot control is independent or classical joint control. In this type of joint control the $n$ joint manipulator to be controlled will be controlled by $n$ decoupled individual joint controllers whose control input is based on the 

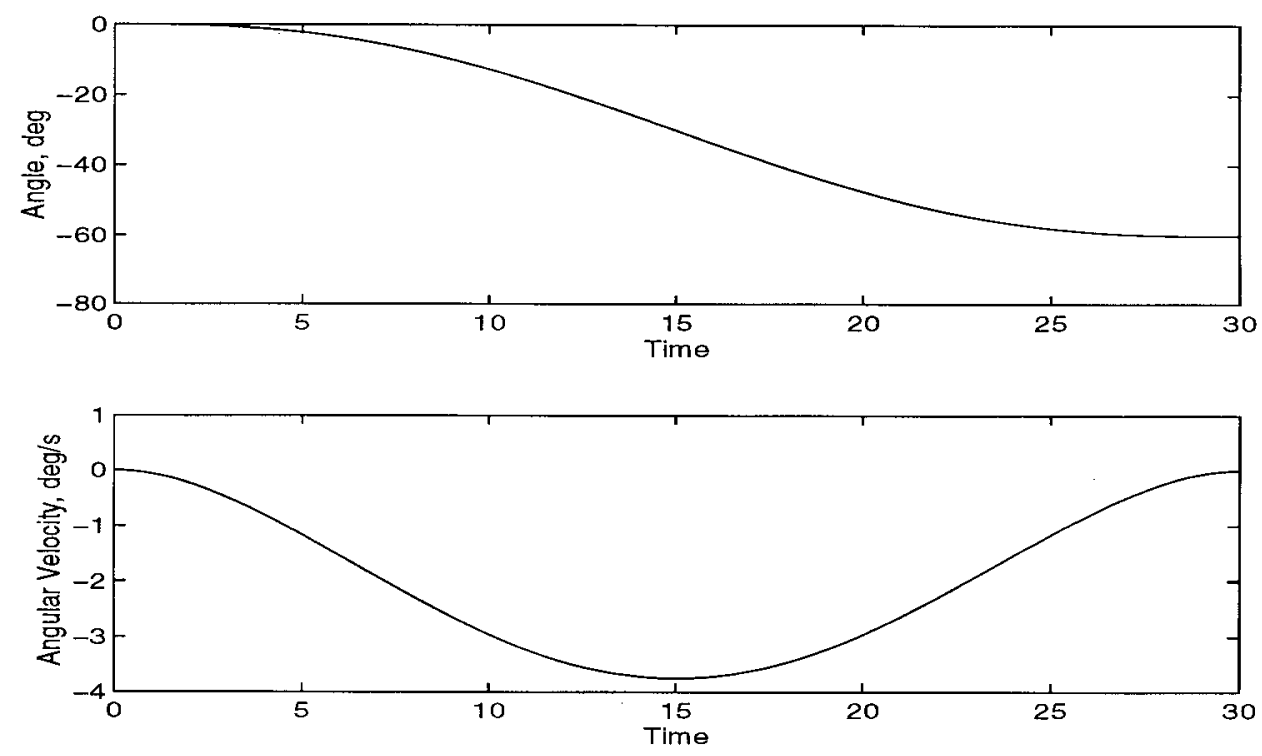

Figure 3.3: Quintic Polynomial Position and Velocity

locally measured joint variables (errors). Independent error driven joint control has long been popular since there is no need to solve complicated nonlinear robot inverse dynamics on-line. It also allows a decoupled analysis of the closed loop system using single-input single-output classical techniques. It has been proven that this type of control is very suitable for following a desired trajectory and in fact is representative of how many industrial robots are controlled today [Crai 86]. It has been proven in [Daws 90] that if PD control is applied to each joint and $e(0)=0$ and $\dot{e}(0)=0$ that the position and velocity errors are bounded within a circle whose radius decreases approximately (for large $K_{v}$ ) as

$$
\frac{1}{\sqrt{K_{v}}}
$$

However, $K_{v}$ cannot be increased without reaching the actuator torque limits. If we define the tracking error of a joint variable to be

$$
e(t)=q_{d}(t)-q(t)
$$


where $q_{d}$ is the desired joint trajectory and $q$ is the actual trajectory, we can define the velocity error to be

$$
\dot{e}(t)=\dot{q}_{d}(t)-\dot{q}(t)
$$

For PD control we would then select our control signal $u(t)$ as proportional-plus-derivative feedback or

$$
u=-K_{v} \dot{e}-K_{p} e
$$

To account for external disturbance and gravity (in the absence of gravity compensation), we add an integral control term of the form $K_{I} \varepsilon$ where

$$
\dot{\varepsilon}=e
$$

The robot arm control torque then becomes

$$
\tau=-u=K_{v} \dot{e}+K_{p} e+K_{I} \varepsilon
$$

If each joint actuator were modelled as having lumped inertia and damping values of $J$ and $B$, the closed loop characteristic polynomial obtained from the error dynamics is

$$
s^{2}+K_{v} s+K_{p}=0
$$

The standard form for the characteristic polynomial is

$$
s^{2}+2 \zeta_{n} \omega_{n} s+\omega_{n}^{2}=0
$$

where $\omega_{n}$ is the joint error natural frequency and $\zeta_{n}$ is the damping coefficient. Therefore the desired joint control performance can be achieved by selecting the appropriate gains. Equating the two polynomials gives

$$
K_{p}=\omega_{n}^{2}
$$

and

$$
K_{v}=2 \zeta_{n} \omega_{n}
$$


To obtain critical damping (no overshoot) for example then

$$
\zeta_{n}=1
$$

and the gains would be related by

$$
K_{v}=2 \sqrt{K_{p}}
$$

The upper limit of the gains are related to the structural frequency of the link, $\omega_{r}$. The first flexible resonant mode of the link is given as

$$
\omega_{r}=\sqrt{\frac{k_{r}}{J_{r}}}
$$

where $J_{r}$ and $k_{r}$ are the link inertia and stiffness respectively. Therefore to avoid exciting the links resonant mode the joint error frequency should be chosen to be less than half the maximum link frequency (configuration dependent usually) or

$$
\omega_{n}<\frac{\omega_{r}}{2}
$$

Of course the joint torque upper limit will also limit the gains.

A $P D$ torque controller was implemented in the simulations, ignoring the integral term because gravity was not used and no disturbance terms were introduced into the system. When the integral term was incorporated it made virtually no difference in the tracking. This controller was very effective in trajectory tracking with large enough gains. Keeping the gains as high as possible suppresses the disturbances from the other robot links. Of course there are many types of more complicated (and effective) control that are described in many different books and papers but for these simulations were not neccesary because of the ideal model assumptions (no gravity, no disturbances, no friction, rigid links). A block diagram of the controller can be seen in Figure 3.4. Further discussion of the gain selections are discussed in Section 4.6.

The control frequency of the controller is the same as the integrator step size frequency. Because a variable step size integrator is used for the simulations (for efficiency), and since 
a control cycle or update occurred at every step, the controller is essentially analog. If the simulation step size was fixed, and for example was set at 0.002 seconds $(500 \mathrm{~Hz})$, then a control frequency less than or equal to $500 \mathrm{~Hz}$ could have been chosen. Having the controller essentially analog will give good control results. If a digital controller had been implemented (as most real time controllers are nowadays), it would have had no effect on the motion control algorithm performance, assuming the control frequency was reasonably fast.

One thing to note is the torque added to the error torque labelled $\tau_{\text {update }}$ in the block diagram. This torque is added to the torque term immediately after a trajectory update occurs. This is because at the beginning of an update (and at $t=0$ ) the joint errors are zero. At the first update step the actual joint trajectories are equal to the (new) desired trajectories and hence there is no torque. Prior to the update there were joint errors since the controller is not perfect and no system can realize infinite gains. What this update torque does is add a constant torque term (for the rest of the movement) equal to the last torque value before the update. The closed loop controller takes care of this offset. If this torque offset is not added, immediately after the torque being zero, there is a very large correction torque which results in trajectory discontinuities. 


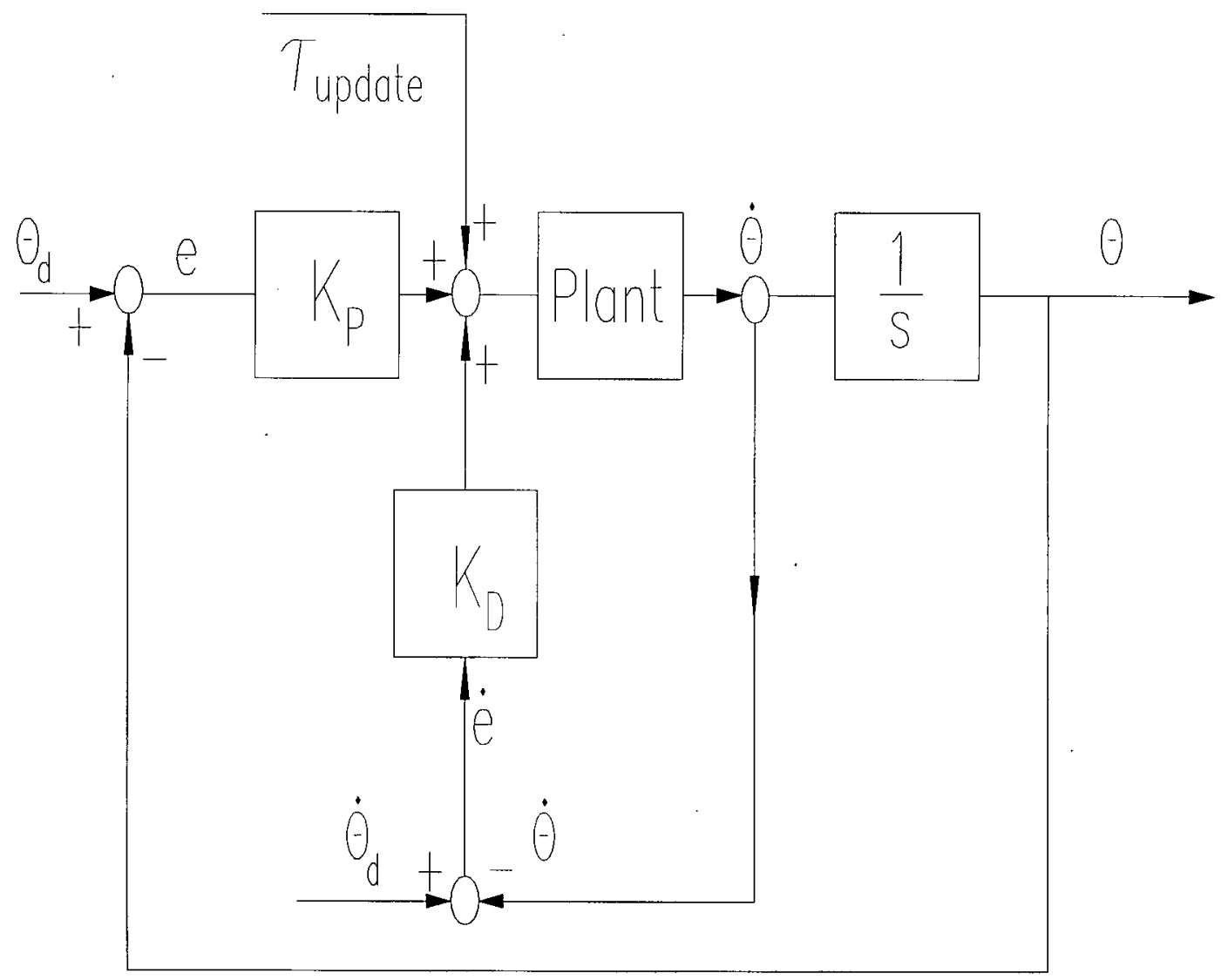

Figure 3.4: Torque Controller 


\section{Chapter 4}

\section{Inverse Kinematics and Trajectory Generation}

\subsection{Review of Inverse Kinematics Methods}

In robotics, finding the end-effector position in Cartesian space given the joint coordinate values is known as forward kinematics and is relatively easy computationally. The more important and difficult problem of finding the joint coordinates $\mathbf{q}=\left(q_{1}, q_{2}, q_{3}, q_{4}, q_{5}, q_{6}\right)$ required to locate the end-effector at a desired position (expressed as $\mathbf{T}^{6}=\mathbf{H}_{(1,6)}$ ) is known as the inverse kinematics problem. The two main methods of finding the inverse kinematics solution are termed the Inverse Transform Technique and the Geometric Approach. In both cases, a closed form solution of the equations is preferred rather than a numerical solution. This is so the inverse kinematics equations can be solved in real-time relative to the control frequency. The closed loop form is also used for developing rules to choose between several solutions when the solution is not unique. In the Inverse Transform Technique, the desired end-effector position or transformation matrix is described in [Fu 87] as

$$
\mathbf{T}^{6}=\mathbf{H}_{(0,1)} \mathbf{H}_{(1,2)} \mathbf{H}_{(2,3)} \mathbf{H}_{(3,4)} \mathbf{H}_{(4,5)} \mathbf{H}_{(5,6)}
$$

A matrix (unknown) is then moved from the right side of the equation to the left side of the equation (known) and the resulting equation is then solved for the unknown parameter. This is repeated until all the joint parameters have a closed loop form.

In the Geometric Approach described in [Fu 87] p.61 the solution is based on geometric 
projections and arm configuration indicators which are functions of joint angles. Arm configuration indicators allow a single solution to be chosen when there are multiple solutions possible. For a six link rotary joint robot with the last three axes intersecting (the wrist), the position vector from the origin to the wrist is projected onto a plane. Trigonometry is then used to solve for the first joint parameter. The second and third joint parameters are solved similarly.

\subsection{Fixed Base Inverse Kinematics}

When a robot is mounted on a fixed base the joint parameters are calculated and passed to the controller which servos the joints to produce the desired end-effector position. The desired parameters or angles are calculated once at the beginning of the movement and they do not change throughout a movement. This is in contrast to the moving base case where the desired endpoint parameters are changing as the base moves (in the robot frame). For a 2 link manipulator the Geometric Approach is used to calculate the endpoint joint parameters. In this simple case the solution reduces to simple trigonometric equations. Let $(x, y)$ be the desired end-effector position in the X-Y plane and let the 2 revolute links have lengths $l 1$ and $l 2$ as seen in Figure 4.1. The following equations then yield the necessary joint angles:

$$
\begin{gathered}
r^{2}=x^{2}+y^{2} \\
C=\cos \left(\theta_{2}\right)=r^{2}-l 1^{2}-l 2^{2} \\
D= \pm \sqrt{1-C^{2}} \\
\theta_{2}=\arctan 2(D, C) \\
\theta_{1}=\arctan 2(x, y)-\arctan 2\left(l_{2} \sin \left(\theta_{2}\right), l_{1}+l_{2} \sin \left(\theta_{2}\right)\right)
\end{gathered}
$$

It is clear that there are two solutions as long as

$$
l_{1}^{2}+l_{2}^{2}<x^{2}+y^{2}
$$




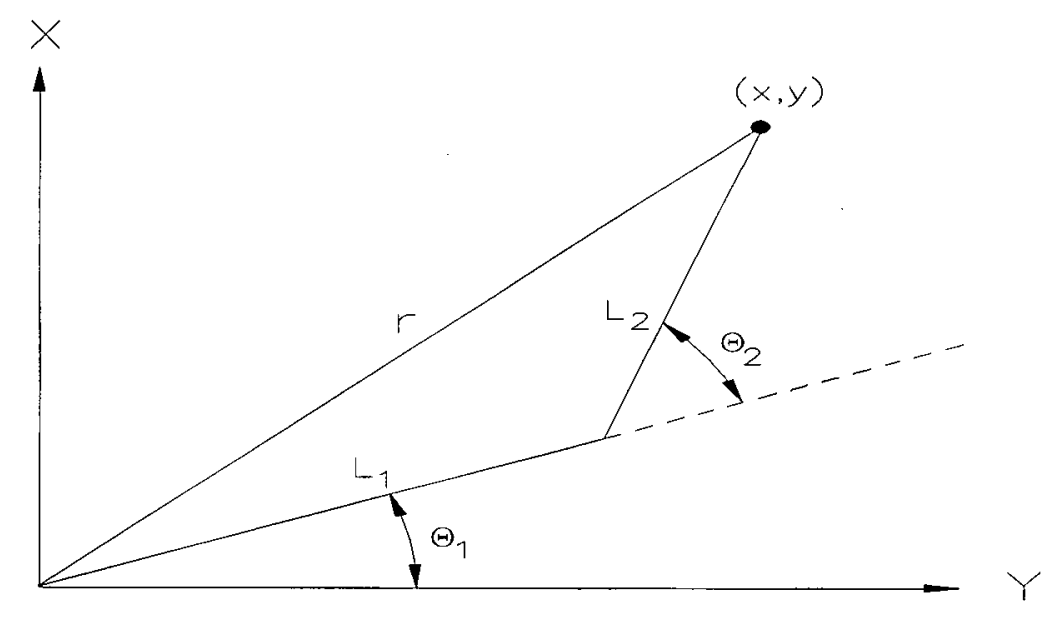

Figure 4.1: Inverse Kinematics for 2-Link Robot

If this is not true then the desired point is not in the robot workspace, and a singularity occurs. Note that the \pm in Equation 4.4 above reveals that the solution is not unique. For this simple 2-D case there are two solutions, each which corresponds to an elbow up or elbow down configuration. In the elbow up configuration both robot links lie above the vector $r$. In the elbow down configuration the links lie below the vector $r$. This shows that the inverse kinematics problem generally has a non-unique solution, the number of possible solutions increasing as the robots degrees of freedom increases. This property can be beneficial for collision avoidance where any number of solutions can provide correct end-effector positioning. The drawback is determining which solution to use if many solutions are possible. 


\subsection{Inverse Kinematics Updating Algorithm for Moving Base}

For the moving base problem, if the required joint angles are calculated at the start of the movement, dynamic coupling will produce motion of the base resulting in end-effector position error. In the algorithm proposed herein, the required joint angles are updated at specified times throughout the movement taking into account the translation and rotation of the base that has occurred since the last update or the start of the movement. These updates are done on-line, assuming the desired end-effector position in the manipulator frame can be measured relatively quickly. Fast measurement feedback would mean that the base does not translate or rotate much during this delay. It is then assumed that the base is fixed at this position for the inverse kinematics calculation which gives new endpoint angles. A new joint trajectory is then calculated (one for each link) and the robot joints are servoed to the new desired final setpoints. This new trajectory has matching boundary conditions as the previous one and is discussed

in Section 4.4. If the desired end-effector position is no longer in the local robot workspace (kinematic singularity), the movement is terminated.

At the end of the movement, if the end-effector is not within the allowable error of the desired position, further end-point servoing is done to complete the movement. When the desired position is reached, the movement is complete.

Computationally, the updating algorithm is fairly simple and could probably be done at a few $\mathrm{kHz}$ on a fast DSP. The computational time depends upon the speed at which a manipulators inverse kinematics and trajectory generation can be completed in. For a planar 2 link robot on a 3 degree of freedom base (as in this thesis), the CPU time between the start of an update (measurement of end-effector position in manipulator frame), and the time when the joints begin servoing to the new angular setpoints, was measured. The time was 0.0078 seconds for all required calculations in a Matlab simulation (inverse kinematics, updating sequence, trajectory generation). The PC used was a Pentium 100 with 32 MB of RAM. On a dedicated 
DSP such as a Texas Instruments C80, the calculation time would likely be 100 times faster. In the computer simulations the updates take only one control cycle or one simulation step to complete. In this sense the update time is instantaneous in the simulation. This is because the inverse kinematics and update algorithm calculations are done before the next integration step starts.

\subsection{Joint Trajectory Updating}

If at the start of a movement the original joint angle required is calculated to be 120 degrees, the robot link begins following a trajectory that will complete this. If at 80 percent of the way through the movement the trajectory is updated and a new final angle of 175 degrees is required, the controller then servos the link along the new trajectory to achieve this. The new endpoint joint angles require that the trajectory polynomials be pieced together with matching boundary. conditions. An example of these angle and angular velocity trajectories can be seen in Figure 4.2 and Figure 4.3. If updates are done at multiple times thoughout the movement after some initial update, the angle and angular velocity trajectories may appear as shown in Figure 4.4 and Figure 4.5. In Figure 4.4 a final joint angle of 120 degrees is required. If at 60 percent of the way through the movement the trajectory is updated, and at 10 percent intervals thereafter $(70,80$, and 90 percent), the final angle required (calculated at the last update or 90 percent) became 183 degrees. In Figure 4.5 it is clear on the 60 percent trajectory where the last two updates occurred. Similar results are seen on the 80 percent trajectory where the first update occurred at 80 percent of the total movement time and then another update again at 90 percent. 


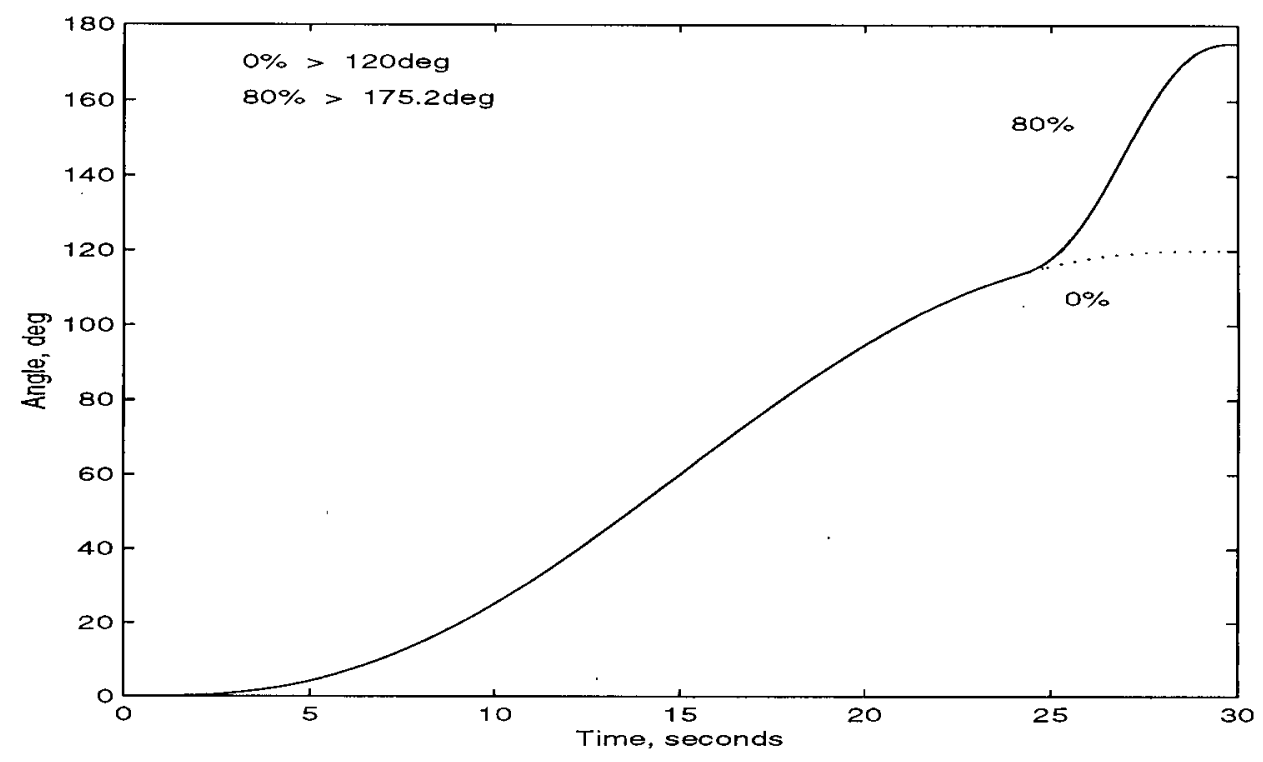

Figure 4.2: Single Joint Trajectory Update - $\theta_{1}$ vs. Time

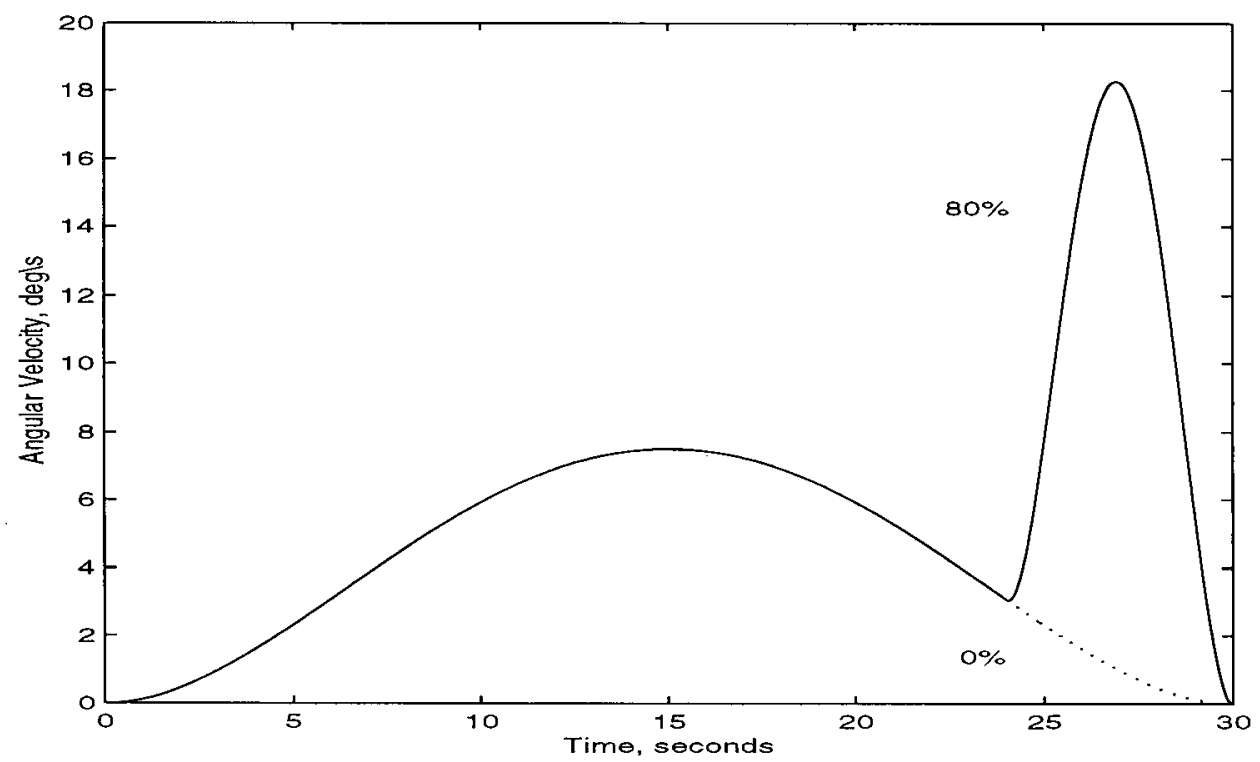

Figure 4.3: Single Joint Trajectory Update $-\dot{\theta}_{1}$ vs. Time 


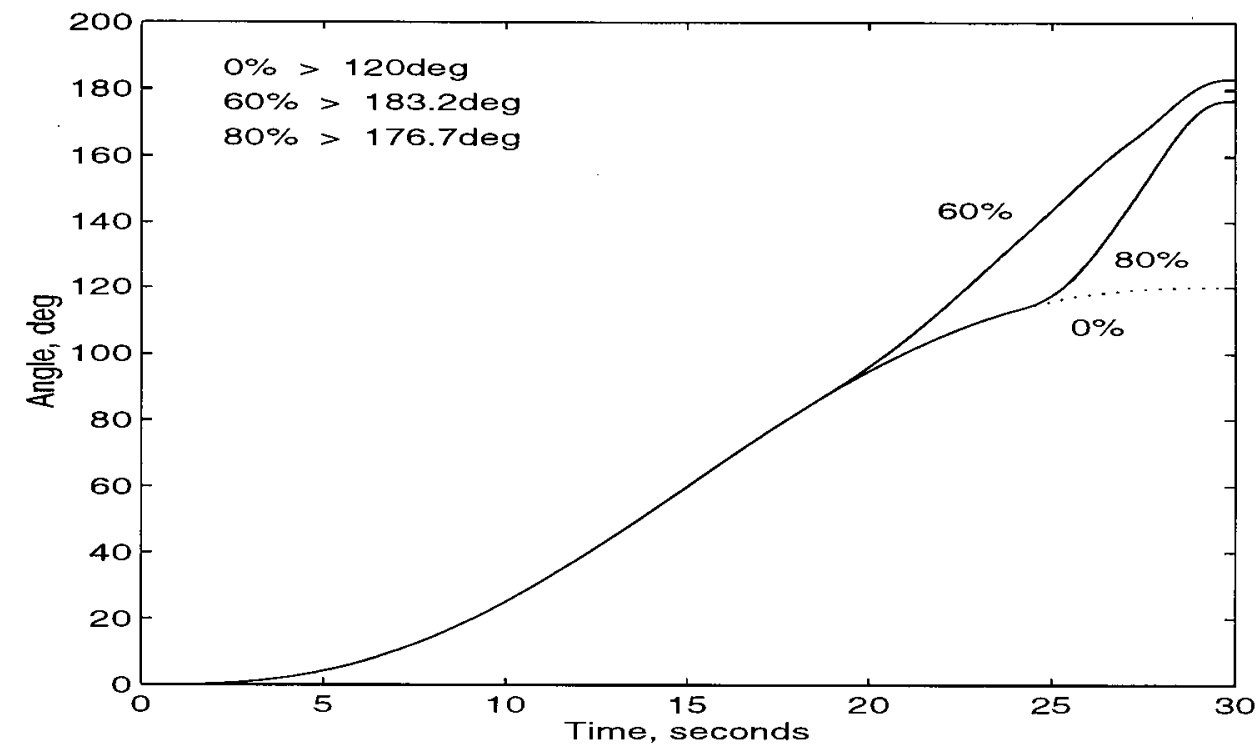

Figure 4.4: Multiple Trajectory Updates $-\theta_{1}$ vs. Time

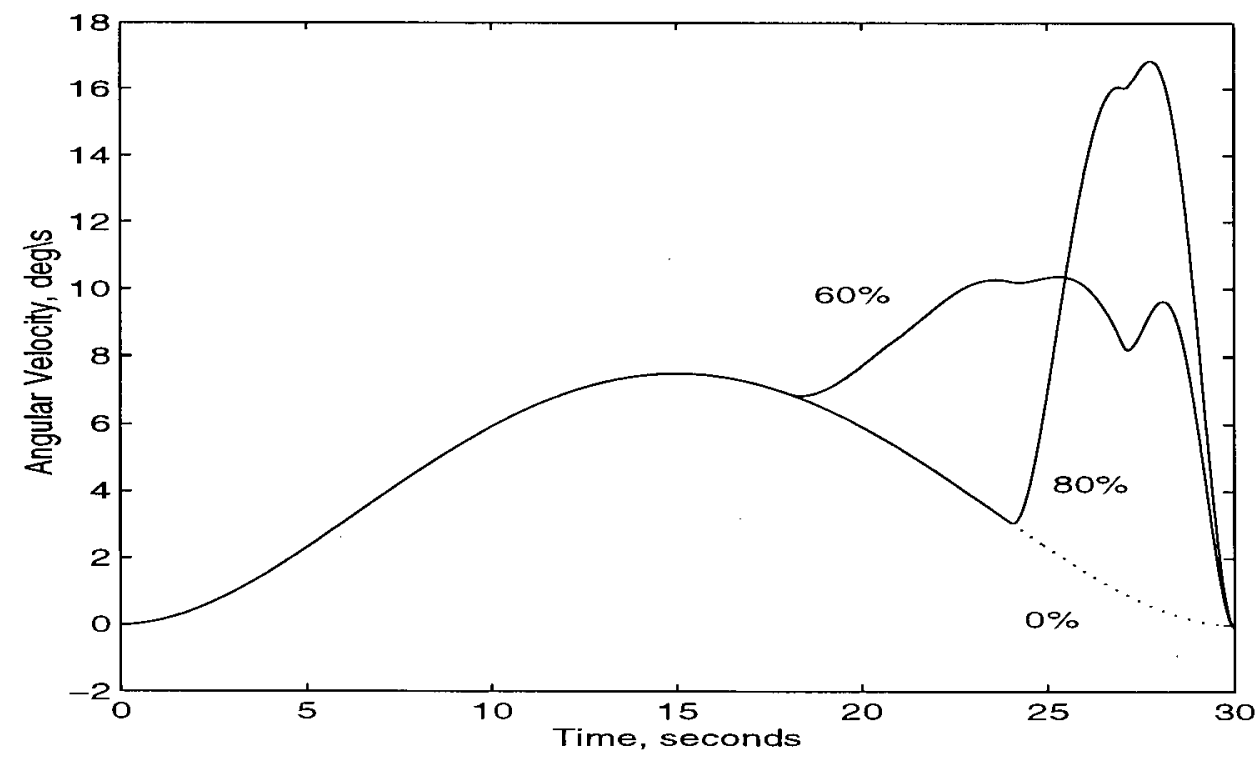

Figure 4.5: Multiple Trajectory Updates $-\dot{\theta}_{1}$ vs. Time 


\subsection{Joint Trajectory Velocity Time Scaling}

There are two reasons that a joint trajectory would have to be time scaled. One of these would be to satisfy the joint velocity constraints and the other would be to satisfy torque constraints. Velocity time scaling is discussed in this section and torque constraints in Section 4.6.1. Each robotic joint has an upper velocity and torque limit that the motor can produce, regardless of whether there is a gear reduction. If the desired joint velocity trajectory exceeds the maximum joint velocity the movement may not result in the desired final setpoint. In the simulations the user may specify a maximum joint velocity that must not be exceeded so that the end-effector velocity or acceleration will not be exceeded. There may also be a minimum specified velocity so that the movement is completed in a specified time. Actual space manipulators typically can move extremely large payloads but at very slow velocities. The CANADARM for example can move a payload many times heavier than itself but with maximum joint velocities of less than $5 \mathrm{deg} / \mathrm{sec}$. To make the simulations as realistic as possible time scaling was implemented. In the Matlab file trajplan.m where the time scaling calculations occur, joint 1 is first time scaled up or down. For each quintic trajectory (coefficients generated in trajgen.m) used, the maximum (or minimum) velocity occurs at the midpoint of the trajectory. If this maximum velocity is greater than the specified one, the time for the next segment (present time until final time) is increased by some specified amount or step. This step size will determine how closely the joint velocity is to the specified velocity. For infinitely small step size increments the velocity will be scaled to exactly the specified maximum velocity. Typically the size used in the simulation was 5 percent of the total time. For scaling the time down to increase the joint velocity (for positive velocities), the total movement time is decreased until the joint velocity is larger than the specified minimum joint velocity. Because joint 1 is scaled first, if the time has been increased, a flag is set so that the joint 2 velocity will not be scaled up thereby decreasing the time. The reasoning behind this is that maximum velocity limits have higher priority than minimum velocity limits. In the case that the joint 2 velocity is scaled, the joint 1 polynomial 
coefficients are recalculated based on the new total movement time. An excellent discussion of exact solutions to torque and velocity scaling can be found in [Holl 84]. The inexact method used herein usually only requires a few loops and gives fairly good results (depends on step size which also affects computational time). A plot of angle and angular velocity trajectories with and without velocity scaling are seen in Figure 4.6 and Figure 4.7. The maximum absolute joint velocity has been set to $5 \mathrm{deg} / \mathrm{sec}$. The end result is that the final desired angle is reached but the movement time has almost doubled from 30 seconds to 58 seconds. The joint maximum velocity constraint of $5 \mathrm{deg} / \mathrm{sec}$ has been met.

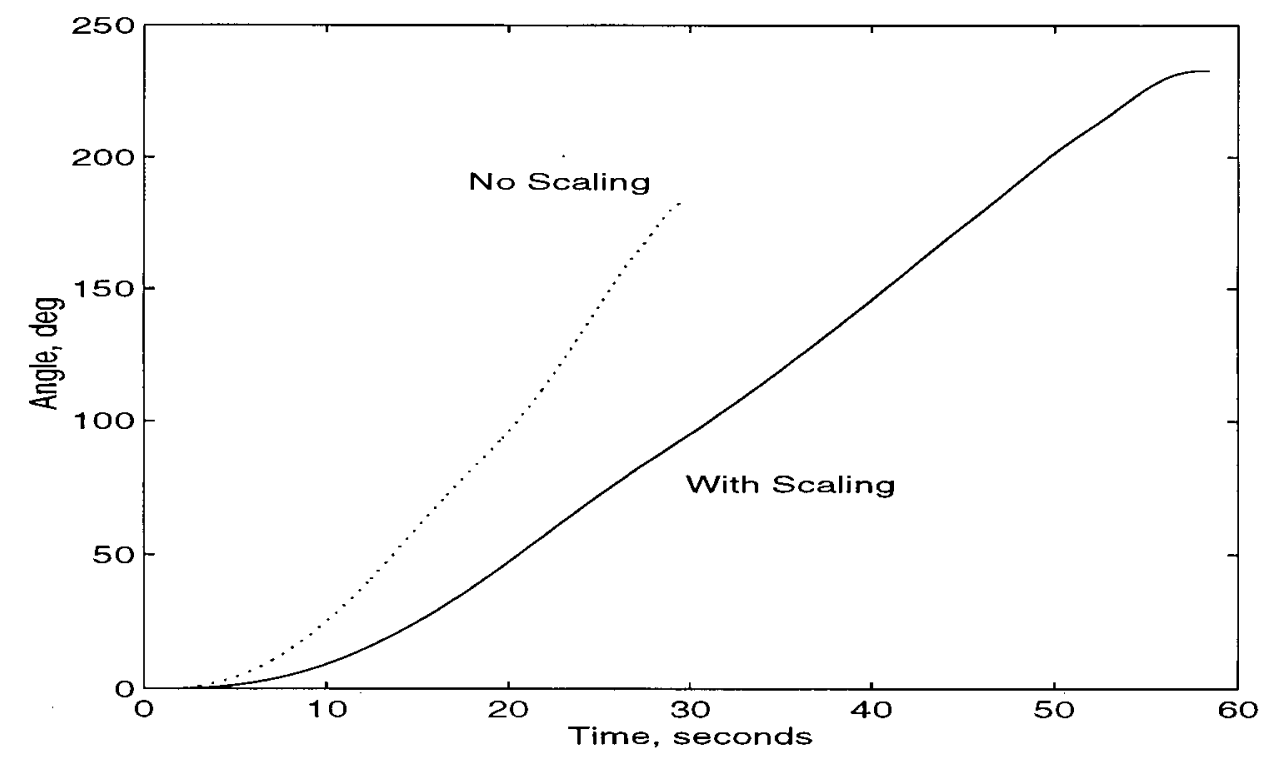

Figure 4.6: Velocity Time Scaling - $\theta_{1}$ vs. Time

\subsection{Joint Servo Control Specifications}

The joint servo gains were based on the theory presented in Section 3.4. A model of a typical link that would be used is based on either a solid rectangular or cylindrical link. It is known 


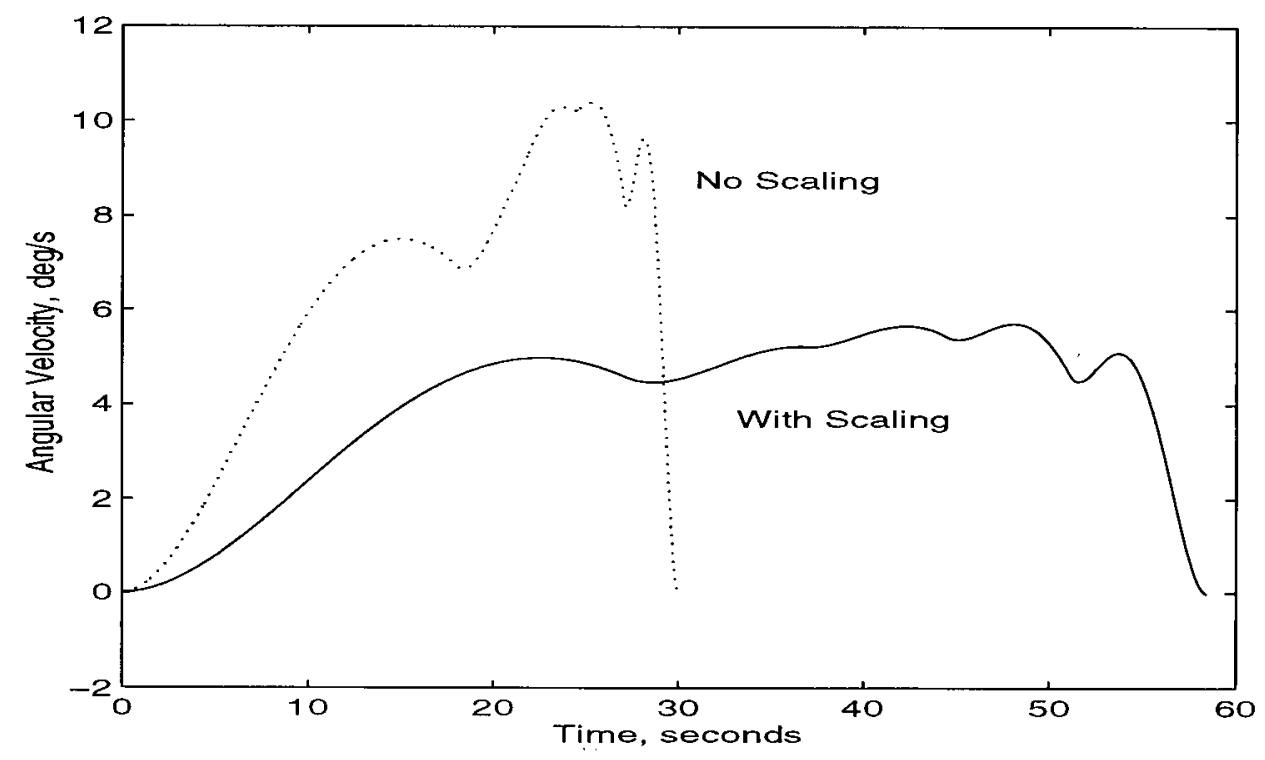

Figure 4.7: Velocity Time Scaling - $\dot{\theta}_{1}$ vs. Time

that hollowed links of the same rectangular or cylindrical shapes are nearly as stiff but lighter than solid ones. A typical industrial robotic link will vary depending on the size, shape, and materials used. The natural frequency chosen based on averages of actual robots is 50 to $60 \mathrm{~Hz}$. Choosing the joint error frequency as half the resonant frequency as given in Equation 3.67, the proportional gain from Equation 3.62 is

$$
K_{p}=\omega_{n}^{2}=\left(\frac{\omega_{r}}{2}\right)^{2}
$$

where $\omega_{r}=(2)(\pi)(55)=346$. The proportional gain is then approximately

$$
K_{p}=30000
$$

and for a critically damped controller the velocity gain is approximately

$$
K_{v}=2 \sqrt{K_{p}}=350
$$

For joint 1 , larger values $\left(K_{p}=40000\right)$ were used because of the increased torque necessary from the effects of joint 2 . These are the values that were used in the controller and provided 
good servoing performance.

\subsubsection{Effect of Torque Actuator Limits}

Torque limits are incorporated to simulate actuator constraints present in all robot actuators. No actuator can produce an infinite amount of torque and so most robot actuators are chosen to produce the necessary maximum torque, and still fall within the size and weight limitations. To model the motor accurately, a motor torque-speed curve as supplied by motor manufacturers would be simulated. In the Matlab controller file ctrlr2.m, if the joint torque at a particular instant exceeds the specified maximum then the torque is set to this maximum joint torque. Consequently there will not be enough torque to servo the robot link angle to the specified angle and the joint error will increase. If the commanded torque is exceeded long enough the joint will not achieve its final desired setpoint. But as the joint error increases when trajectory updating is occurring, at each update larger and larger joint velocities will be required. When the required velocity for next trajectory or part of the movement exceeds the maximum permissible joint velocity, velocity time scaling will occur. As discussed in Section 4.5, this serves to decrease the required maximum joint velocity which will also reduce the required joint torque. Therefore torque time scaling is indirectly occurring through velocity time scaling. Exact torque scaling is not performed since torque control is not being used, and a simple actuator limit is sufficient for the simulations.

To set approximate actuator torque limits we observe the link dynamic equations of motion. In the absence of gravity the torque term for each joint is a sum of the inertial and CoriolisCentripetal terms resulting from each joint and its coupling with other joints and the base. We will assume for torque limits that we are dealing with a fixed two link robot and substitute the masses, link lengths, and angular velocities and accelerations into the dynamic equations. Taken from any robotics textbook or as derived by Maple when checking the robot-base dynamic 
equations in Section 3.2.4, the equations for the two link robot are

$$
\begin{gathered}
\tau_{1}=\left[\frac{1}{3} m_{1} l_{1}^{2}+m_{2}\left(l_{1}^{2}+\frac{1}{3} l_{2}^{2}+2 l_{1} l_{2} \cos \left(\theta_{2}\right)\right)+m_{p}\left(l_{1}^{2}+l_{2}^{2}+2 l_{1} l_{2} \cos \left(\theta_{2}\right)\right)\right] \ddot{\theta}_{1}+ \\
+\left[m_{2}\left(\frac{1}{3} l_{2}^{2}+\frac{1}{2} l_{1} l_{2} \cos \left(\theta_{2}\right)\right)+m_{p}\left(l_{2}^{2}+l_{1} l_{2} \cos \left(\theta_{2}\right)\right)\right] \ddot{\theta}_{2}- \\
\left(m_{2}+2 m_{p}\right)\left(l_{1} l_{2} \sin \left(\theta_{2}\right) \dot{\theta}_{1} \dot{\theta}_{2}-\left(\frac{1}{2} m_{2}+m_{p}\right)\left(l_{1} l_{2} \sin \left(\theta_{2}\right) \dot{\theta}_{2}^{2}\right.\right. \\
\tau_{2}=\left[\left(\frac{1}{3} m_{2}+m_{p}\right) l_{2}^{2}+\left(\frac{1}{2} m_{2}+m_{p}\right) l_{1} l_{2} \cos \left(\theta_{2}\right)\right] \ddot{\theta}_{1}+ \\
\left(\frac{1}{3} m_{2}+m_{p}\right) l_{2}^{2} \ddot{\theta}_{2}+\left(\frac{1}{2} m_{2}+m_{p}\right) l_{1} l_{2} \sin \left(\theta_{2}\right) \dot{\theta}_{1}^{2}
\end{gathered}
$$

The value for joint maximum velocity is chosen as $10 \mathrm{deg} / \mathrm{sec}$ and the maximum angular acceleration as $250 \mathrm{deg} / \mathrm{sec} / \mathrm{sec}$. If the link lengths are 2 metres each and the masses 100 kilograms each, after converting angular values into radians we get

$$
\begin{gathered}
\tau_{1_{\text {max }}}=4340 \mathrm{Nm} \\
\tau_{2_{\text {maxt }}}=2046 \mathrm{Nm}
\end{gathered}
$$

At the beginning of each simulation the torque limits are calculated (in the Matlab initialization file initvars.m) given the current simulation parameters but using the above angular velocity and acceleration limits. It should be noted that because of the relatively low angular velocity and large angular acceleration most of the torque is due to inertial forces and very little due to Coriolis and centripetal acceleration torques. These angular parameters are very typical of a high gear ratio robotic joint that is capable of low speeds but high torques (with harmonic drives for example). Because of the relatively long simulation times for the runs, the maximum torques developed were much lower than the prescribed upper limits. If we set the maximum torques arbitrarily low we can see the difference it makes when velocity time scaling occurs. The angles, angular velocities, and torques of a movement with no scaling, with torque scaling, and with torque and velocity scaling are seen in Figure 4.8, Figure 4.9, and Figure 4.10. This movement has trajectory updating occurring at every 5 percent of the total movement time, 
starting at 60 percent of the movement time. For the no scaling movement, the movement is completed in 5 seconds without regard to torque or joint velocity limits (only last part of movement shown for detail). Note that in this movement a relatively large torque is required at the end of the movement to obtain the desired angle and to decrease the angular velocity to zero (always an endpoint condition). If the actuator torque limit is set to $150 \mathrm{Nm}$, the desired angle is not reached and the joint velocity is not zero at the end of the movement. This is because the torque limit is reached and there is not enough torque to follow the desired trajectories. This torque saturation can be seen clearly in Figure 4.10. If velocity time scaling is enabled we see the joint velocity is zero at the end of the movement and also that the actuator torque constraint has been satisfied.

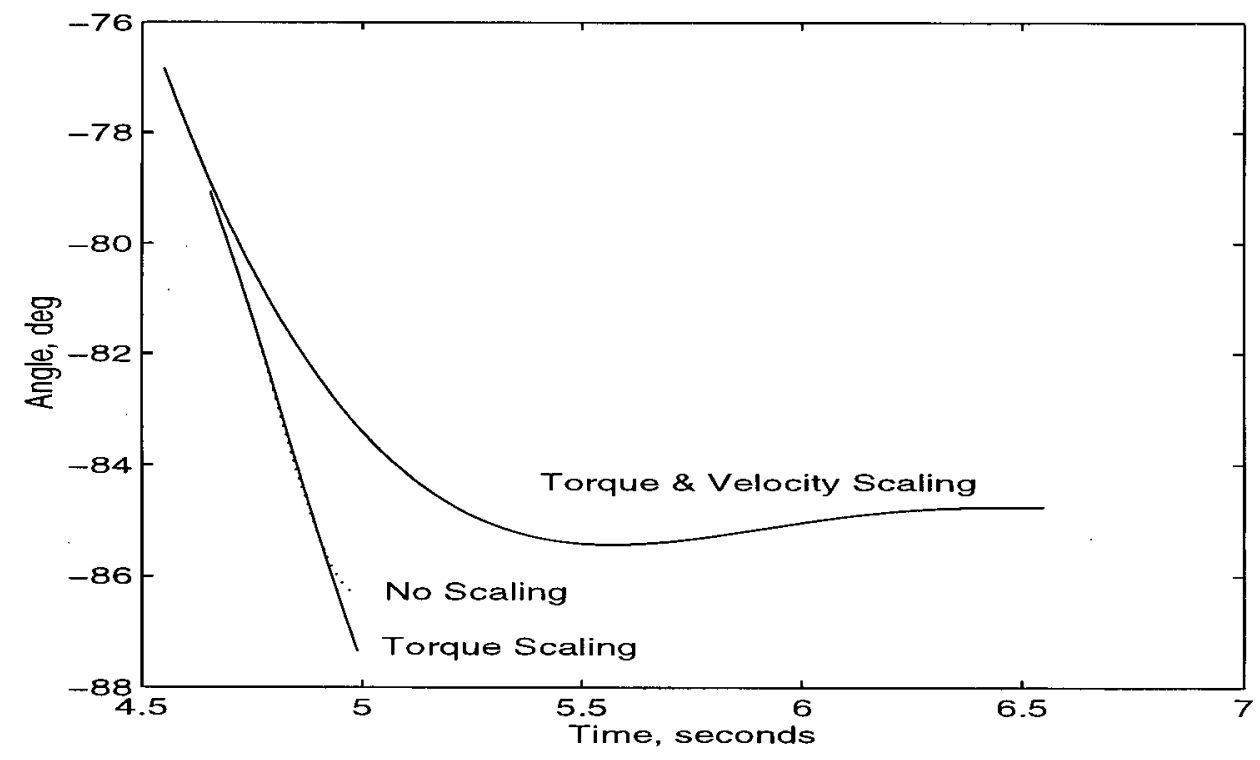

Figure 4.8: Torque Limits $-\theta_{1}$ vs. Time 


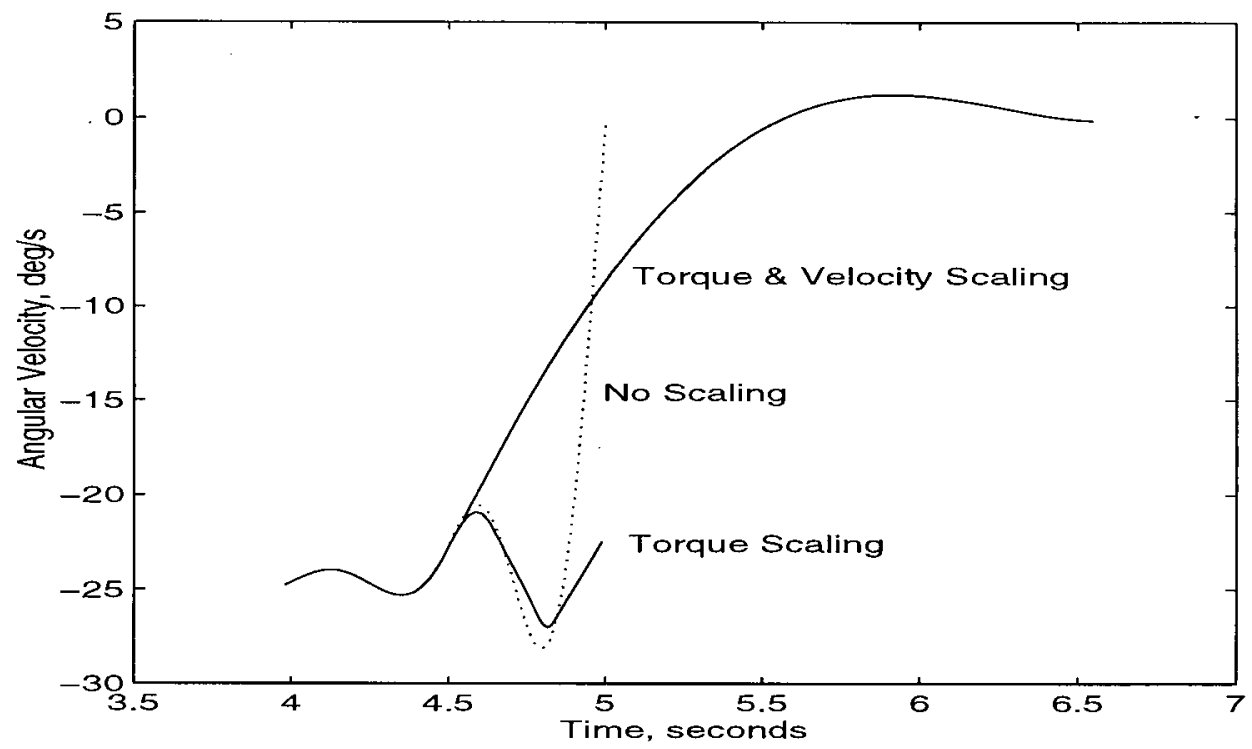

Figure 4.9: Torque Limits $-\dot{\theta}_{1}$ vs. Time

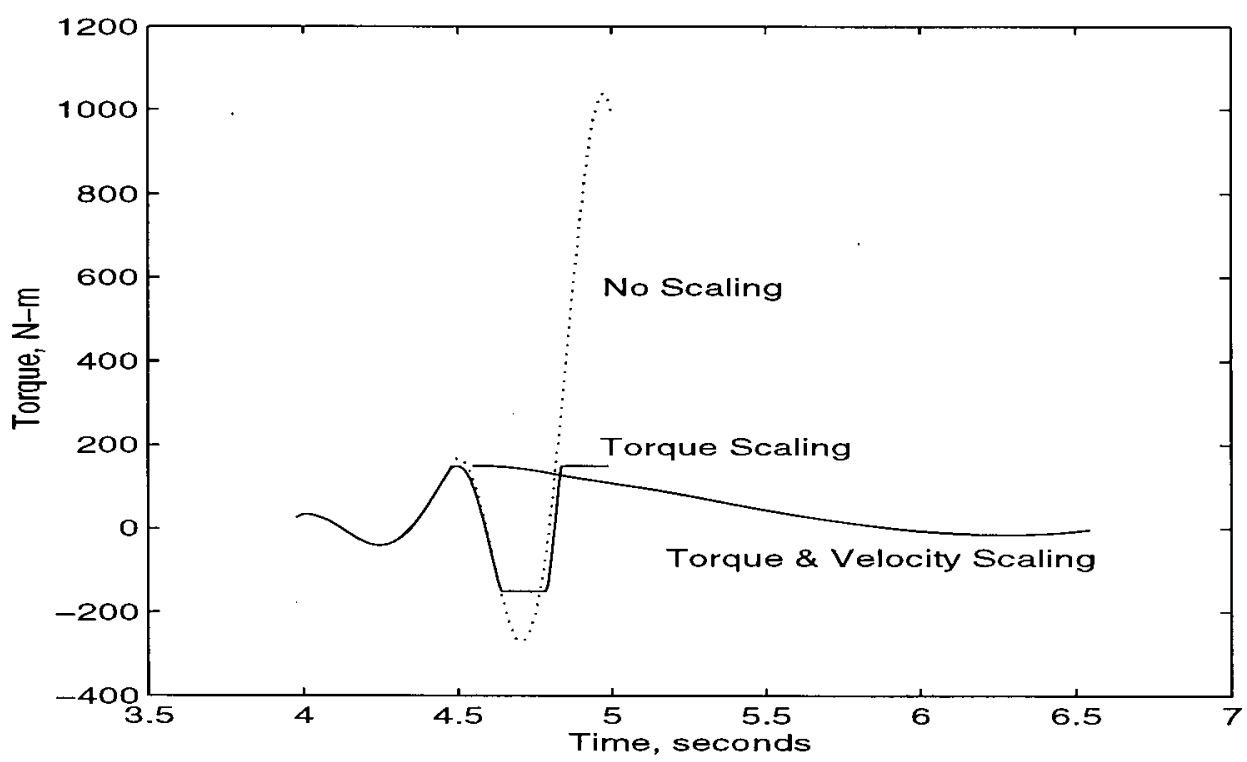

Figure 4.10: Torque Limits - Joint Torque vs. Time 


\subsection{Space Manipulator Workspaces and Dynamic Singularities}

Unlike singularities for fixed-base manipulators that are solely a function of kinematics, space manipulators without base motion control encounter dynamic singularities. These singularities occur when the end-effector cannot move in some inertial direction, and are a function of the dynamic properties of the manipulator and base. Dynamic singuarities occur when the system generalized Jacobian, $J^{*}$ as defined in [Papa 93], becomes rank deficient or non-invertible. The Jacobian for a manipulator-base system relates the end-effector's linear and angular velocities in inertial space, to the controlled manipulator joint angles. Unlike fixed-base Jacobians, this generalized Jacobian depends upon dynamic parameters as well as kinematic ones. The principles in this section were developed by E. Papadopoulus and S. Dubowsky, and can be found in [Papa 93] and [Papa 90]. A good reference for general spacecraft dynamic and control principles is found in [Hugh 86]. Dynamic singularities are path dependent, depending upon the history of the spacecraft attitude. And because of the dynamic coupling in the system, the spacecraft attitude depends upon the history of the manipulator motion. This path dependence is due to the non-integrability of the angular momentum of the system. Physically, each point in the manipulator workspace can be reached with an infinite number of system configurations. Therefore a point may or may not be singular depending upon the path used to get there.

The workspace for a space manipulator is therefore related to the system's dynamic singularities. The standard manipulator kinematic singularities are discussed in Section 4.1. When the spacecraft attitiude is controlled (e.g. with gyroscopes), the manipulator workspace is termed the Reachable Workspace, and is a sphere centered at the system centre of mass. This represents a maximum workspace for the manipulator-base system. The workspace in which dynamic singularities can occur is termed the Path Dependent Workspace (PDW). Points in this space can only be reached if a suitable path is taken. The difference between the Reachable Workspace boundary and Path Dependent Workspace is temed the Path Independent Workspace (PIW). 
Points in this workspace can always be reached, and can never lead to dynamic singularities. Graphical examples of these terms are illustrated in Figure 4.11.

To illustrate these terms, points $\mathrm{A}$ through $\mathrm{D}$ are labelled in Figure 4.11. This figure and the following example are taken from [Papa 93] (Figure 7, page 50). Because points B and D both lie in the $P I W$, either point can be reached from the other, or from any point found in the region labelled PIW. Point $\mathrm{A}$ or $\mathrm{C}$ can also be reached from any point (movement) in the $P I W$. But a point such as $\mathrm{A}$ in the $P D W$ may or may not be reachable directly from point $\mathrm{C}$, or from another point in the $P D W$. When the term directly is used here, it means directly as in a straight line or direct type path. But this does not mean that the point can never be reached. The manipulator may have to perform repetitive movements (i.e. where the end-effector traces out a closed loop path repetitively) to re-orient the base. A discussion of this technique can be found in [Papa 90]. The manipulator may then be able to move to point $\mathrm{A}$ directly from this new configuration. At a dynamic singularity, the end-effector may still be able to move, just not in certain directions. To maximize the $P I W$, manipulator redundancy may be utilized if it exists.

Because calculation of the dynamic singularities and the various workspaces depend upon all the dynamic parameters of the system, these concepts were not incorporated into the motion control algorithm or the trajectory generator developed in this thesis. The main principle behind developing the motion control or updating algorithm in this work, is that the dynamic parameters of a system need not be known. This is true, except for the problem of dynamic singularities. And as discussed previously, for many systems in space, values for these paramters may be very difficult to obtain.

The one type of singularity that is implemented in this thesis, is the standard fixed-base manipulator kinematic singularity. If this type of singularity is reached, the movement is terminated. When a dynamic singularity is encountered, this is not explicitly known by the 
algorithm. Consequently, joint servoing continues until a kinematic singularity is reached. In the simulation results presented in the next chapters, a point is unreachable if it is no longer in the local manipulator workspace. The point still may be reachable using a suitable path in conjunction with the workspace principles discussed in this section.

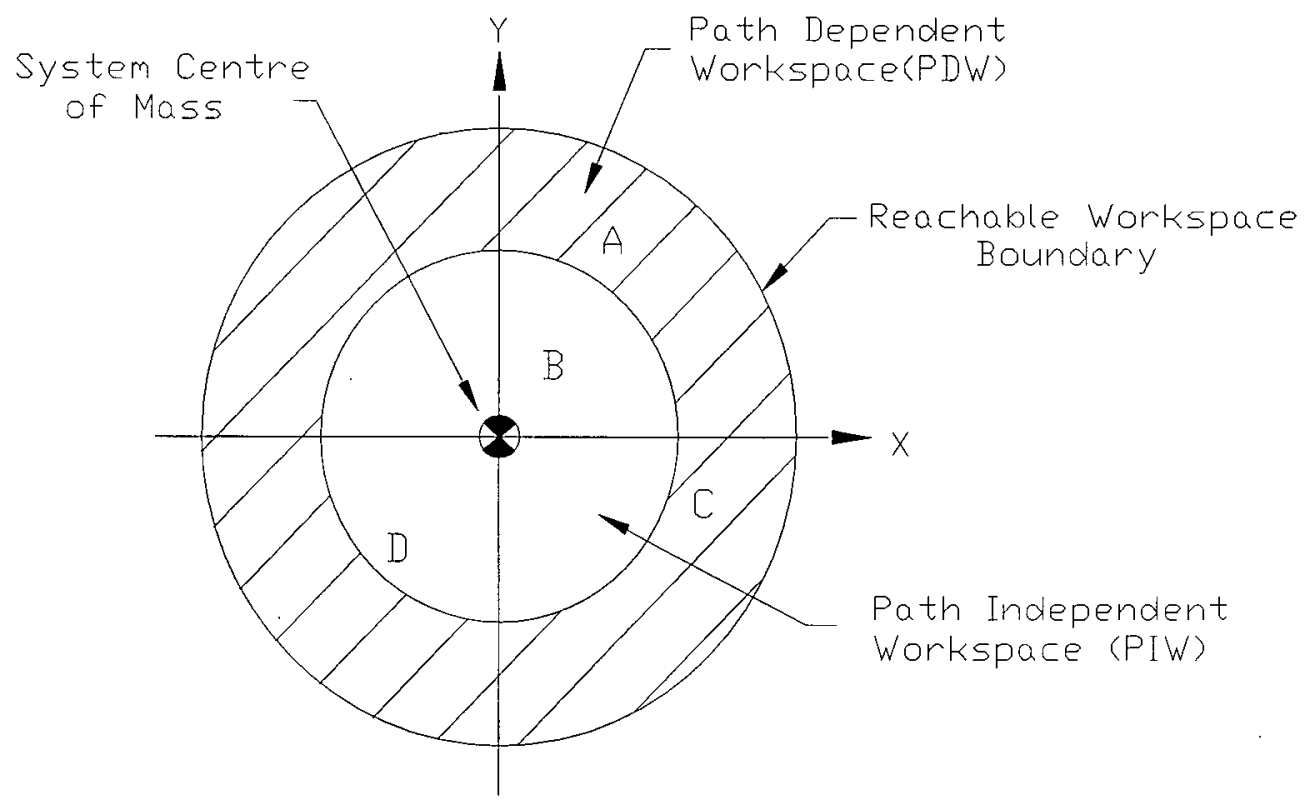

Figure 4.11: Space Manipulator Workspaces 


\section{Chapter 5}

\section{Dynamic Simulation Results}

\subsection{No Updates}

In most of the simulations in this chapter, the robot links are both 2 meters long, have masses of 100 kilograms each, and are modeled as solid bars for inertia purposes. The base is 2 meters by 2 meters square and has a mass of 600 kilograms in the simulations. The payload is assumed to be a point mass at the end of the second robot link but is set to a zero point mass in this thesis. The entire system is assumed to be at rest at the beginning of the simulations, and therefore comes to rest at the end of the movement (link velocities of zero). Non zero robot or base initial conditions can be specified but were not used in any simulations shown in this thesis. The simulations were done using SI units, but for the plots the lengths are shown in $\mathrm{cm}$. When a movement occurs without any trajectory updating, the end-effector of the robot will (if the controller is tuned properly) reach the desired position in the robot coordinate frame (fixed) but not in the inertial frame. The amount by which the end-effector misses the desired position (inertial frame) will depend on the size of the commanded joint movements and the base-arm mass (and inertia) ratio. If the mass ratio of the base to arm (termed $R_{M}$ ) is very large then the system is essentially a fixed base system. The amount of base disturbance will depend on the $R_{M}$ as well as on how fast the movement is completed. Figure 5.1 shows a fixed base movement (dotted) and a non-fixed base movement (solid), with the trajectory of the moving base shown as dash-dot. The manipulator initial configuration is shown by the 
dashed lines and is fully extended perpendicular to the base. The initial end effector position is $(500,100) \mathrm{cm}$ in the inertial frame and $(400,0) \mathrm{cm}$ in the robot base frame $\left(H_{0}\right)$. The circles in the plot show the end-effector initial and final positions. In both cases the end-effector of the robot reaches $(-200,200) \mathrm{cm}$ in the robot base frame $\left(H_{0}\right)$. But for the non fixed base case the robot-base has rotated about 60 degrees in the inertial frame. Figure 5.2 shows the joint angles and angular velocities for fixed and moving base robots. Figure 5.3 shows the movement of the base (coordinate frame $H_{B}$ ) in the $\mathrm{x}$ and $\mathrm{y}$ directions. In Figure 5.4 the trajectory of the base (position and velocity) and the base angle and angular velocity are shown. Note that the system is at rest at the end of the movement.

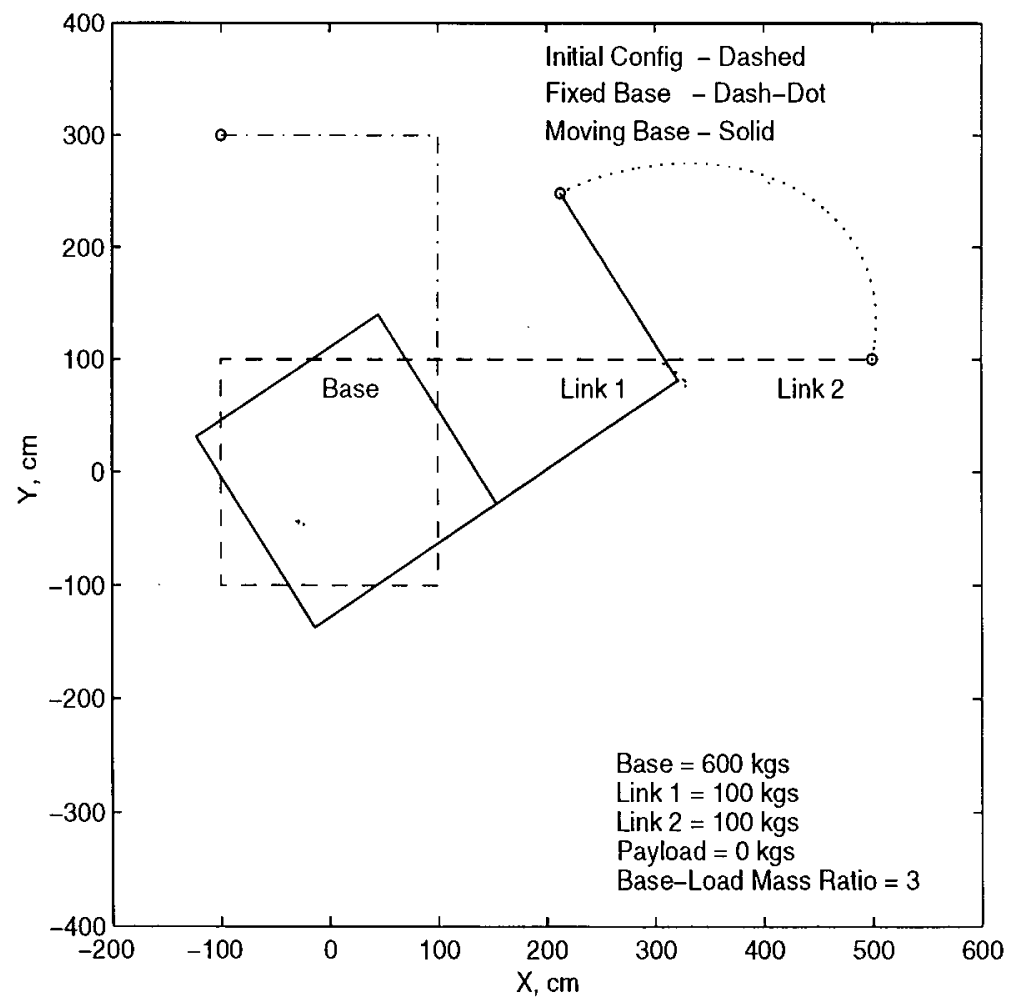

Figure 5.1: Fixed and Moving Base System Trajectories . 

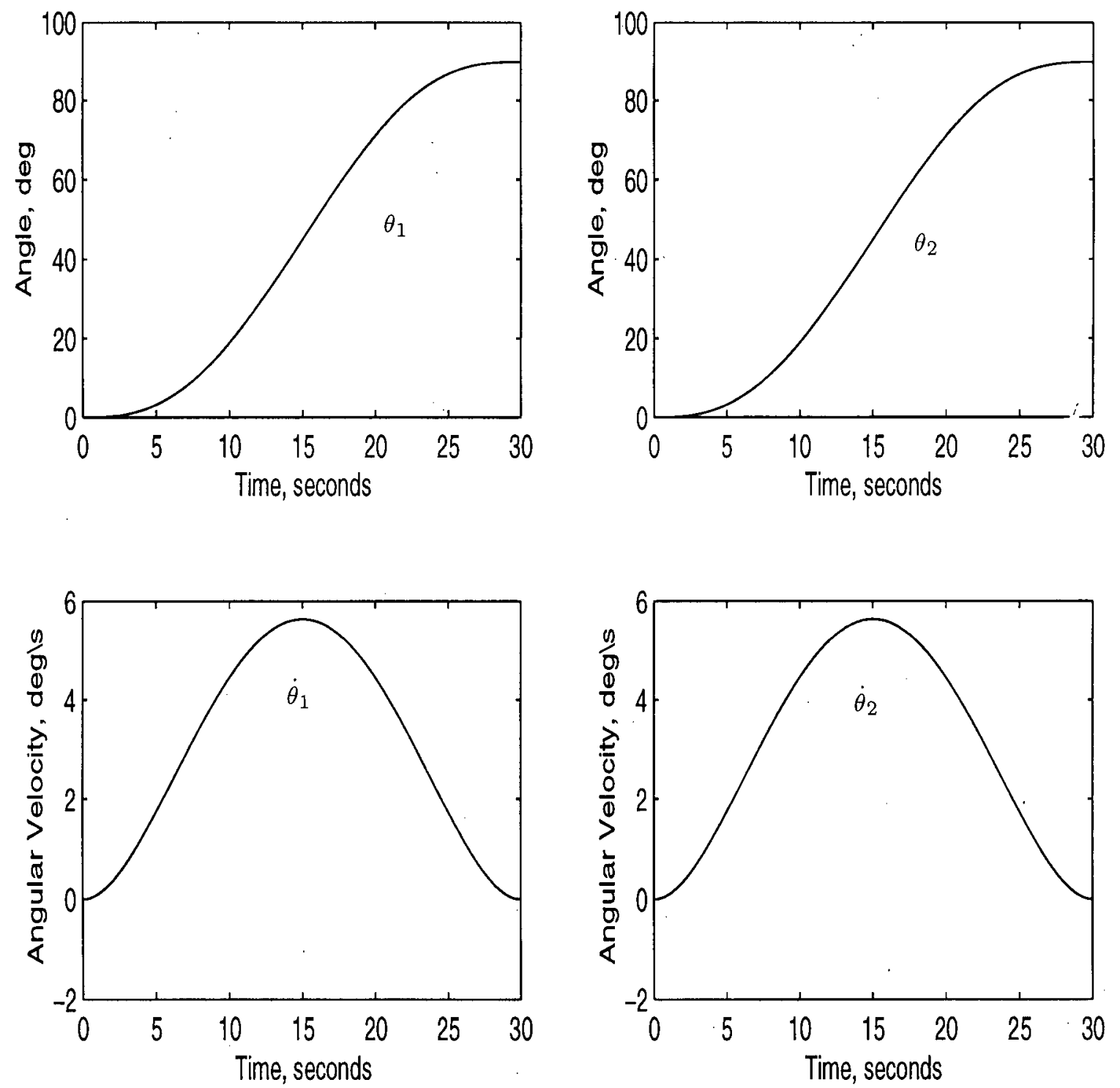

Figure 5.2: Fixed and Moving Base Joint Angle and Angular Velocity 

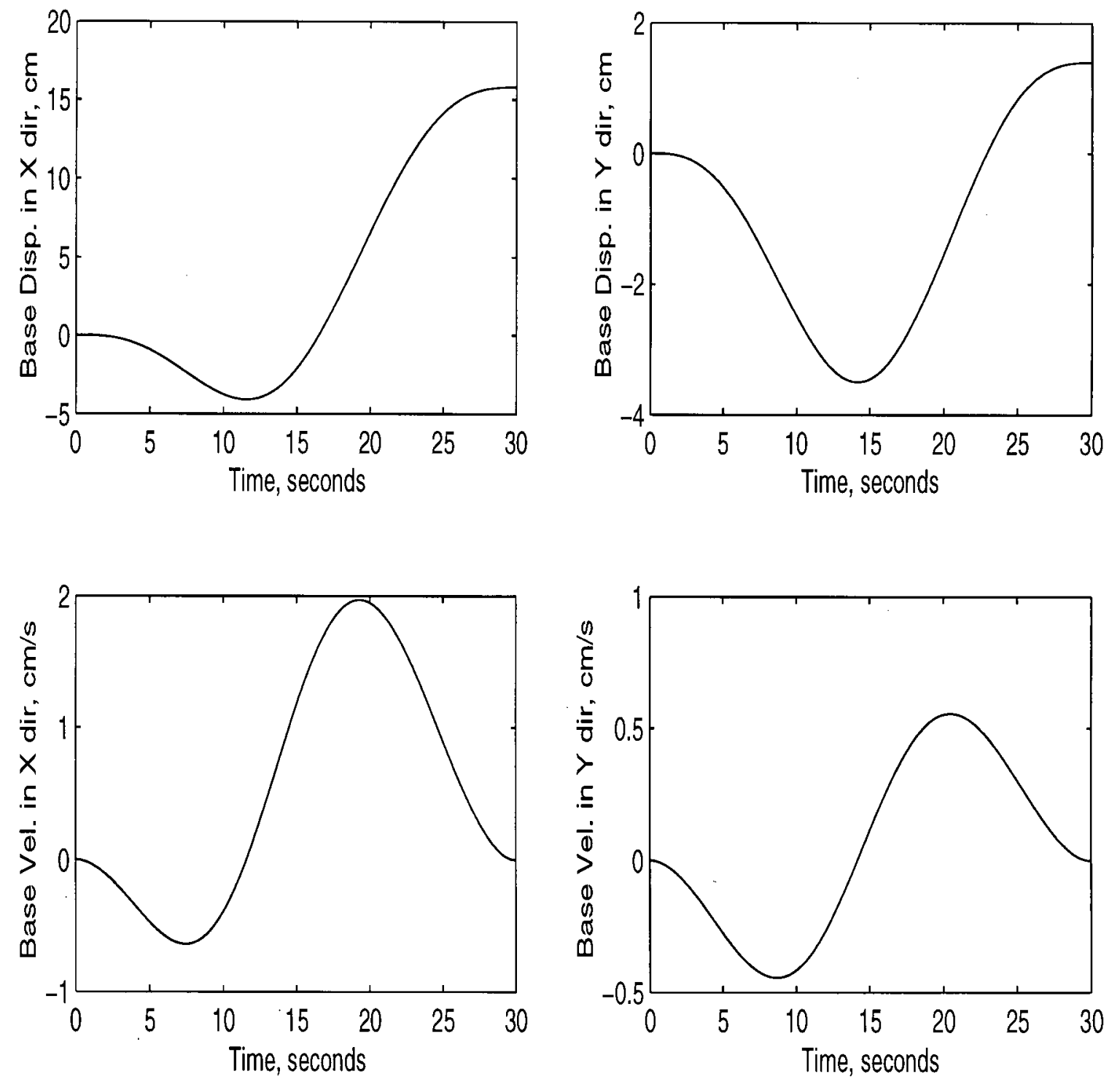

Figure 5.3: Moving Base Position and Velocity 

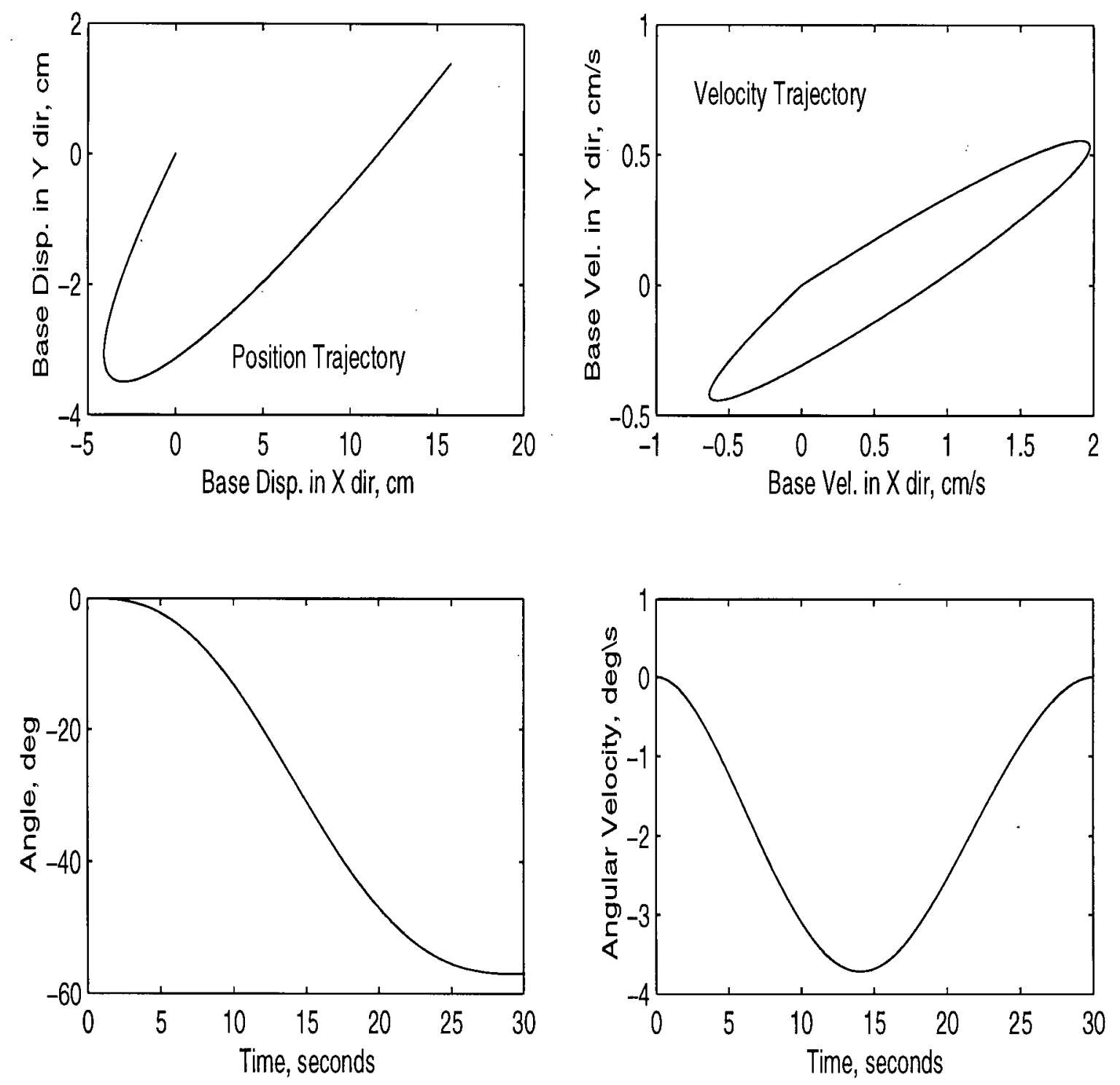

Figure 5.4: Moving Base Angles, Angular Velocities, System Trajectories 


\subsection{Effect of Mass - Inertia Ratio}

One of the main factors to be considered in a system is the mass ratio between the base and the robot (and payload if any). A system with a very heavy base relative to the robot, or a large base-arm mass ratio $\left(R_{M}\right)$ will not be affected as much by base movement as a system with a small $R_{M}$. Another ratio that can be used is the inertia ratio. This may be more relevant in some cases as the inertia ratios taken about the robot base frame incorporate the distance of the masses from the point being considered. If we are to use a ratio as a variable to be considered for updating and as a guide for the end-effector error magnitude, the mass ratio may be used as an average. An example where the inertia ratio may be more useful would be a very short, heavy robot (relative to the base) or a very light robot with a heavy payload. For this case if we assume the robot mass to be centered at between $1 / 3$ to $1 / 2$ the length of the robot, which would on average be the case, there will be less end-effector error than expected. Therefore using the mass ratio is conservative. For the heavy payload case the inertia ratio would be a better choice. Figure 5.5 shows the end-effector trajectories for mass ratios varying between 3 and 100 with the $R_{M}$ 's labeled.

\subsection{Effect of Total Movement Time and Time Scaling}

The total time that the manipulator takes to complete its movement has no effect on the final manipulator position or end-effector final error in the robot or inertial frame. And because all time scaling does is increase or decrease the total movement time, it also has no effect on end-effector final error. When the movement time is lengthened to meet joint torque or velocity limits, the result is reduced joint torques and accelerations and therefore less base acceleration. If a maximum allowable base acceleration was specified, time scaling could be used to reduce base acceleration by increasing the movement time. 


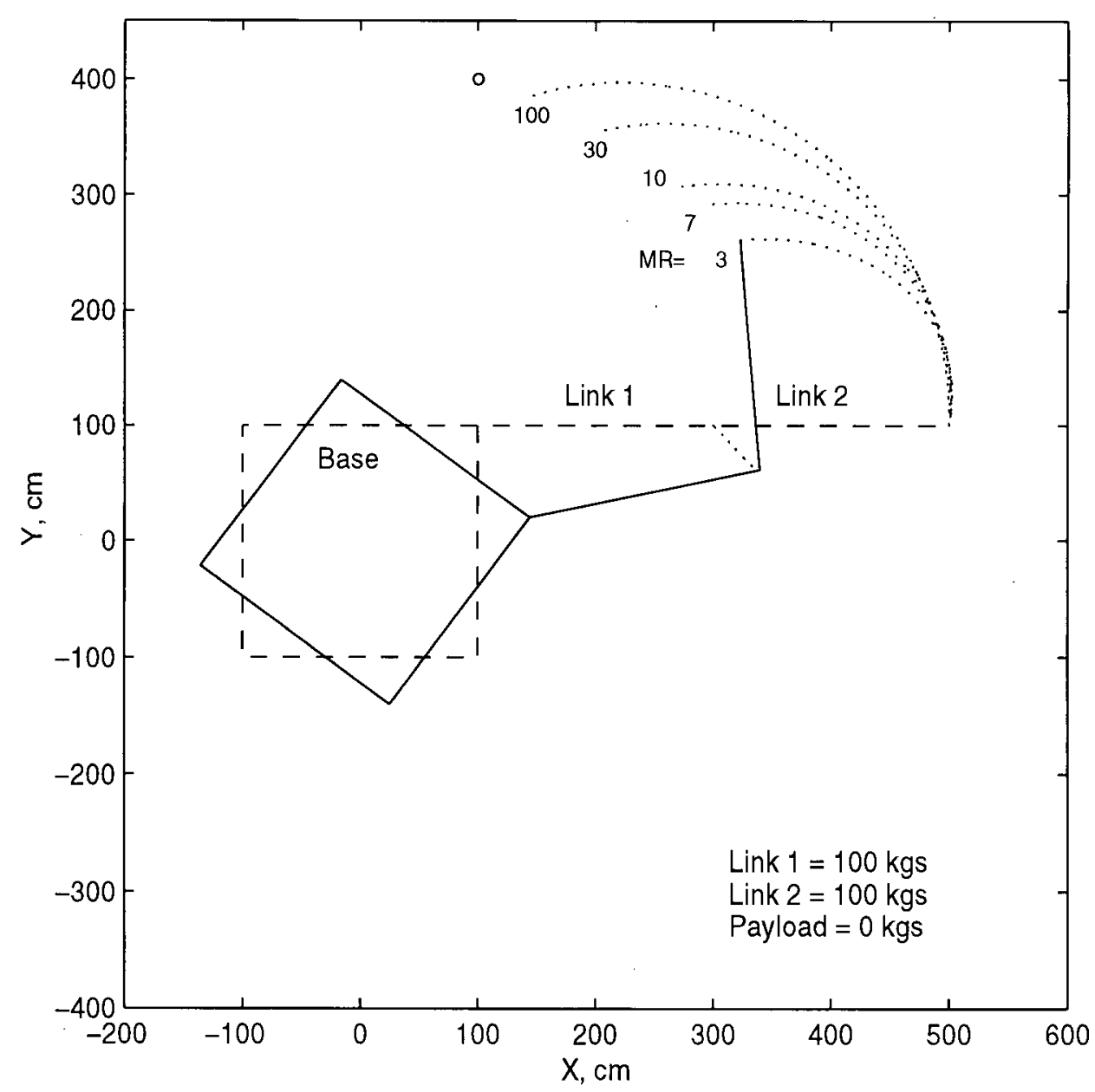

Figure 5.5: End-Effector Trajectories - Variable Mass Ratio 


\subsection{Effect of Single Updates}

In this section the effect that a single update (and the time at which it occurs) has on the final end-effector error will be investigated. It is intuitive that if an inverse kinematics re-calculation is performed at the very beginning of a movement, only a small portion of base movement will have occurred and hence recalculating the new desired end-effector position (in the robot frame) will not result in much improvement over no updating at all. It would also make sense that updating at the last minute when there is a large error would probably be best, except for the fact that a large, fast robot movement may be required and hence large required velocities and torques would be required (these two predictions are exactly what is observed during the simulation). This would not be a problem if velocity scaling occurred which would have the effect of increasing the simulation. But during this last large movement a corresponding large base disturbance would occur resulting in end-effector error. Therefore we enter the cycle of cause and effect, not unlike the Uncertainty Principle in classical physics. Informally summarized, when an observer takes a measurement of a system, the observer interacts with the system and therefore disturbs the system. But in most cases this disturbance can be ignored for all intents and purposes depending on the required accuracy of the measurement. One example of where disturbances cannot be ignored are typically measurements of sub-atomic particles.

The analogy in our system is that whenever the robot moves, regardless of the $R_{M}$, there is a corresponding system re-orientation about the system centre of mass. This is why, in theory, we can never reach the desired end-effector position. This is similar to the example often given in mathematics where, if one were to move from point A to point B with each step being only one-half (or less than) the distance of the previous step, it is impossible to attain point B. But in this example and in our robot-base system, it is later shown that we can get as close as is necessary to meet any required accuracy (whether a single or multiple updates) using the motion control algorithm. 
Figure 5.6 shows the system for a movement with and without an update. Without an update, the robot achieves its commanded position in the robot frame but in the inertial frame misses the desired point by approximately $190 \mathrm{~cm}$. If an update is performed at $80 \%$ of the total movement time (25 sec of a $30 \mathrm{sec}$ movement), the error is reduced to $100 \mathrm{~cm}$. Figure 5.7 shows end-effector trajectories of movements at $80 \%, 60 \%, 40 \%$ and $0 \%$ (no update). Note the axes here are not to scale. Clearly, the later the update the lower the error. Figure 5.8 shows the

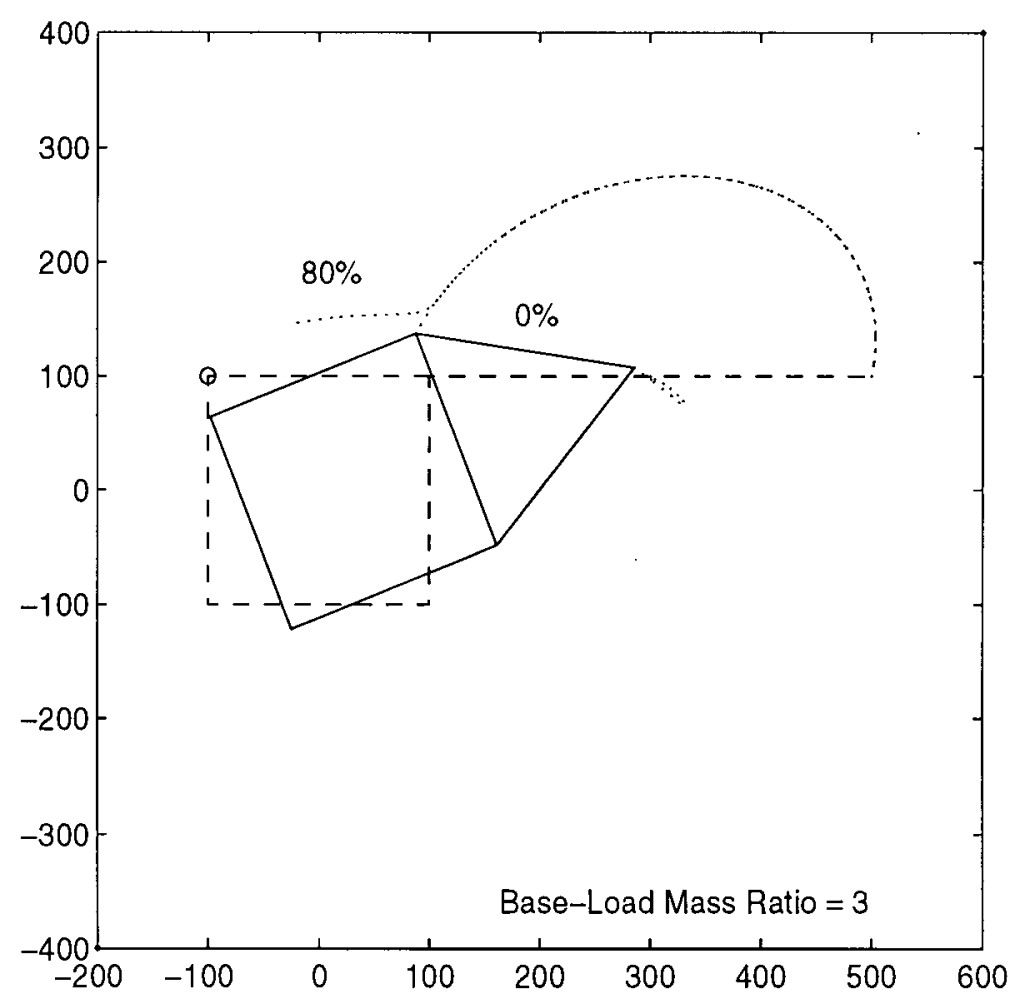

Figure 5.6: Single Update End-Effector Trajectories

joint angles for the different update times. In this plot the final desired angle increases at each update. Figure 5.9 and Figure 5.10 show the joint angular velocity and torque. The later the update the larger the required velocities and torques. In these simulations there is no velocity scaling or torque limits so that all movements take the same time to complete. Figure 5.11, Figure 5.12, and Figure 5.13 show the joint angle, angular velocity, and torque for joint 2. The torque ripples seen in Figure 5.13 at the update points are due to the fact that the controller is 


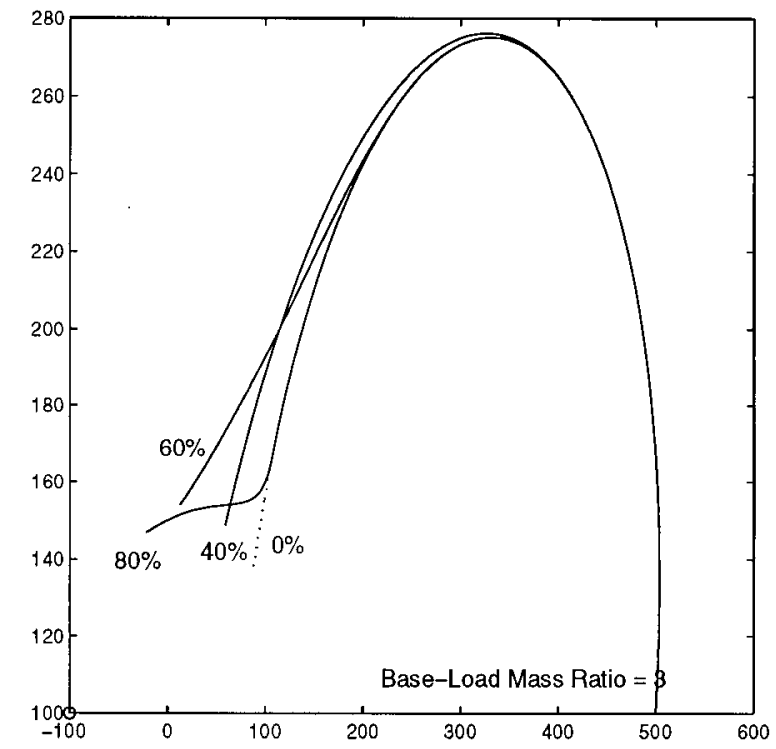

Figure 5.7: Single Update End-Effector Trajectories Close Up

not perfect. Even though a constant update torque is added at an update, the torque still jerks slightly at an update. It is small enough that its effect cannot be seen on the angle or angular velocity plots.

Figure 5.14 shows the $\mathrm{x}$ and $\mathrm{y}$ position and velocity of the base for movements updated at 0 and $80 \%$ while Figure 5.15 shows the base position and velocity in the xy plane for the two updates. There is more base movement when an update occurs. In Figure 5.16 the base angle $(\phi)$ for the different update times are seen. In this plot at each update the final desired angle decreases. Figure 5.17 shows the base angular velocity. Figure 5.18 shows the robot end-effector error in $\mathrm{cm}$ versus the update start time as a percentage of the total movement time. Each curve in the plot represents a different desired position in the robot base frame, with the original end-effector position being $(400,0) \mathrm{cm}$. Six representative positions were chosen in the robot workspace. The circular points on the curves are the final error (in $\mathrm{cm}$ ) for one entire (30) second movement with the single update done at that time. All these simulations were done with the previous robot parameters and a $R_{M}$ of 3 . A total of 96 simulations were 


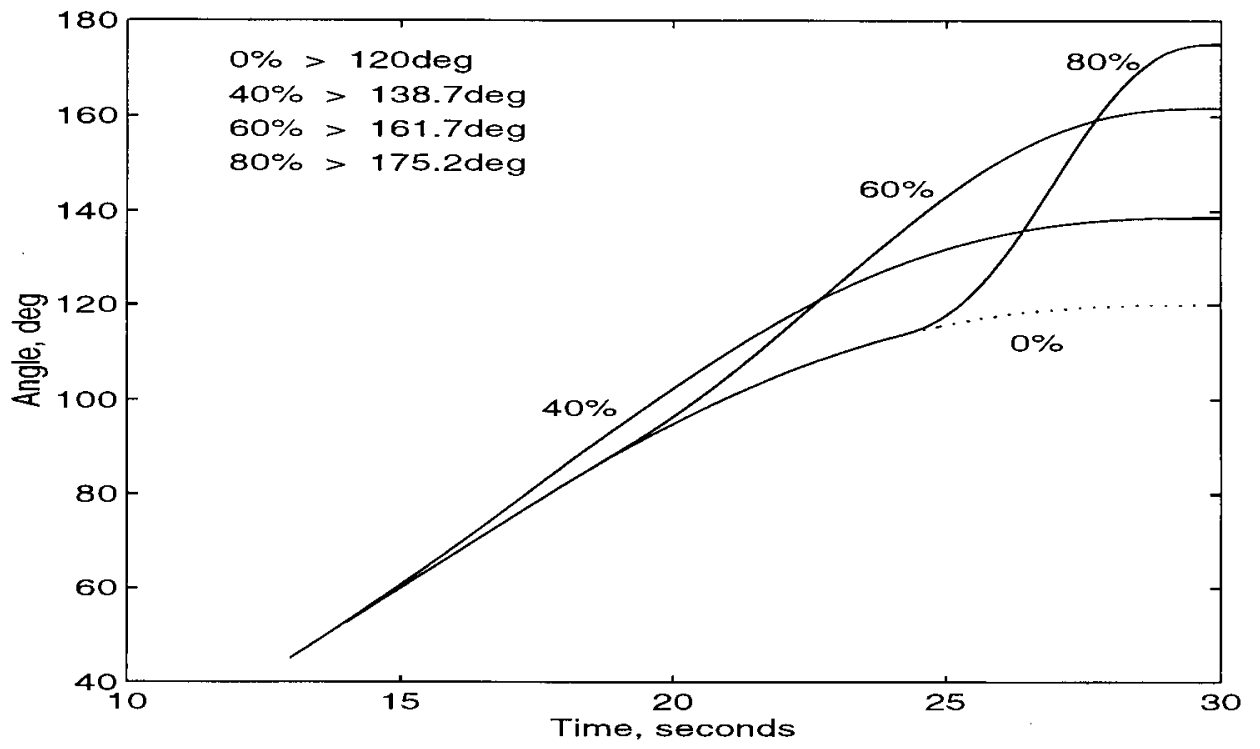

Figure 5.8: Single Update $\theta_{1}$ vs. Time

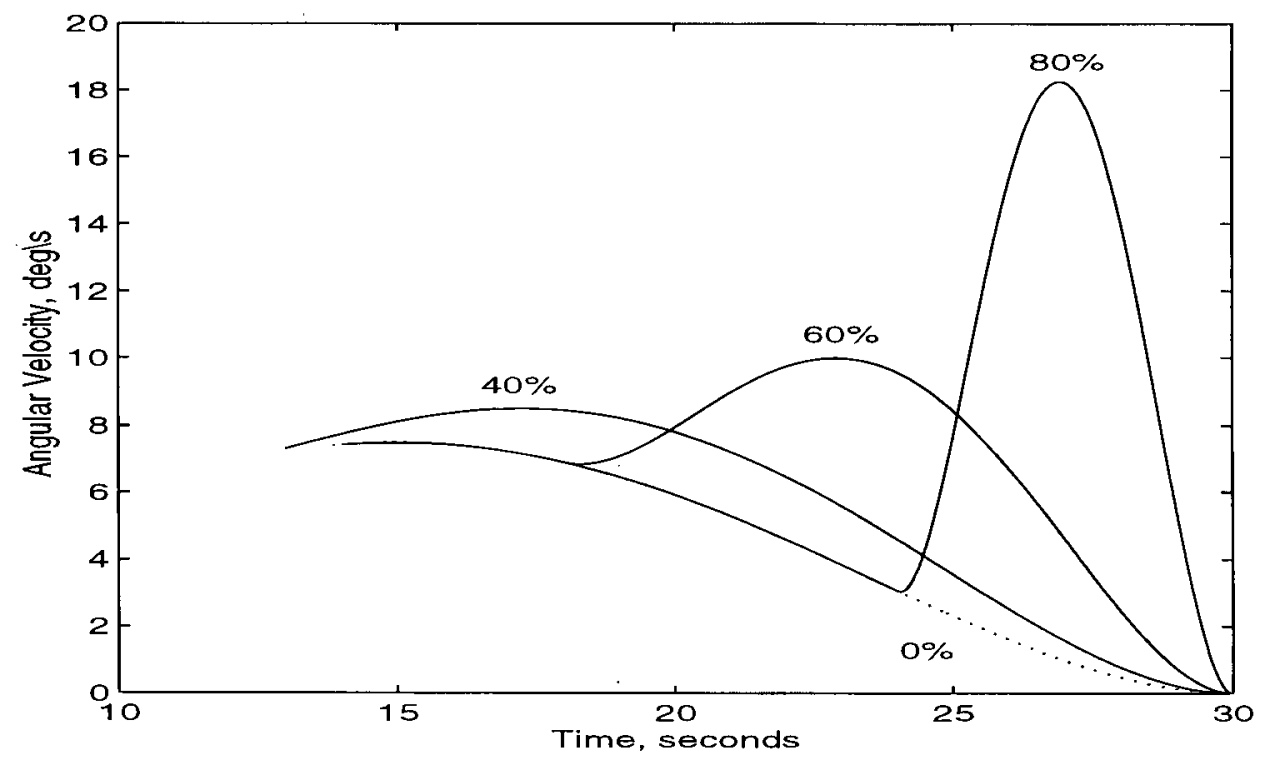

Figure 5.9: Single Update $\dot{\theta}_{1}$ vs. Time 


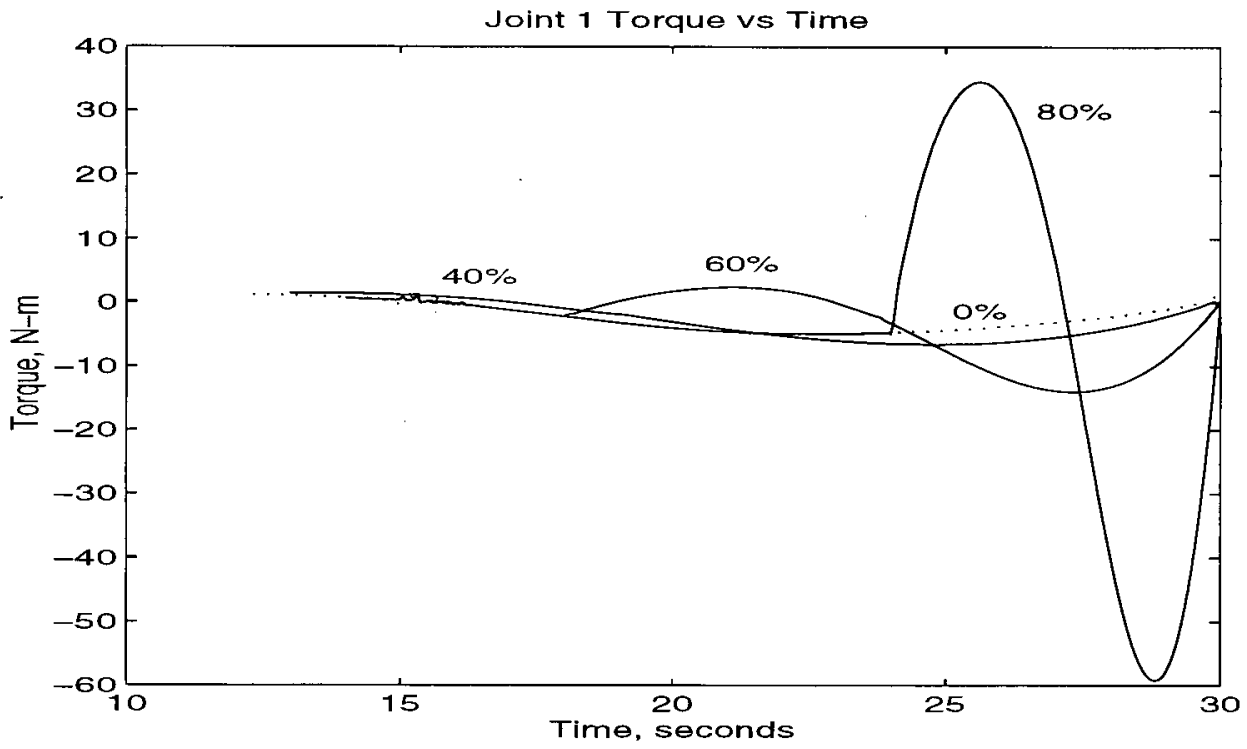

Figure 5.10: Single Update Joint 1 Torque vs. Time

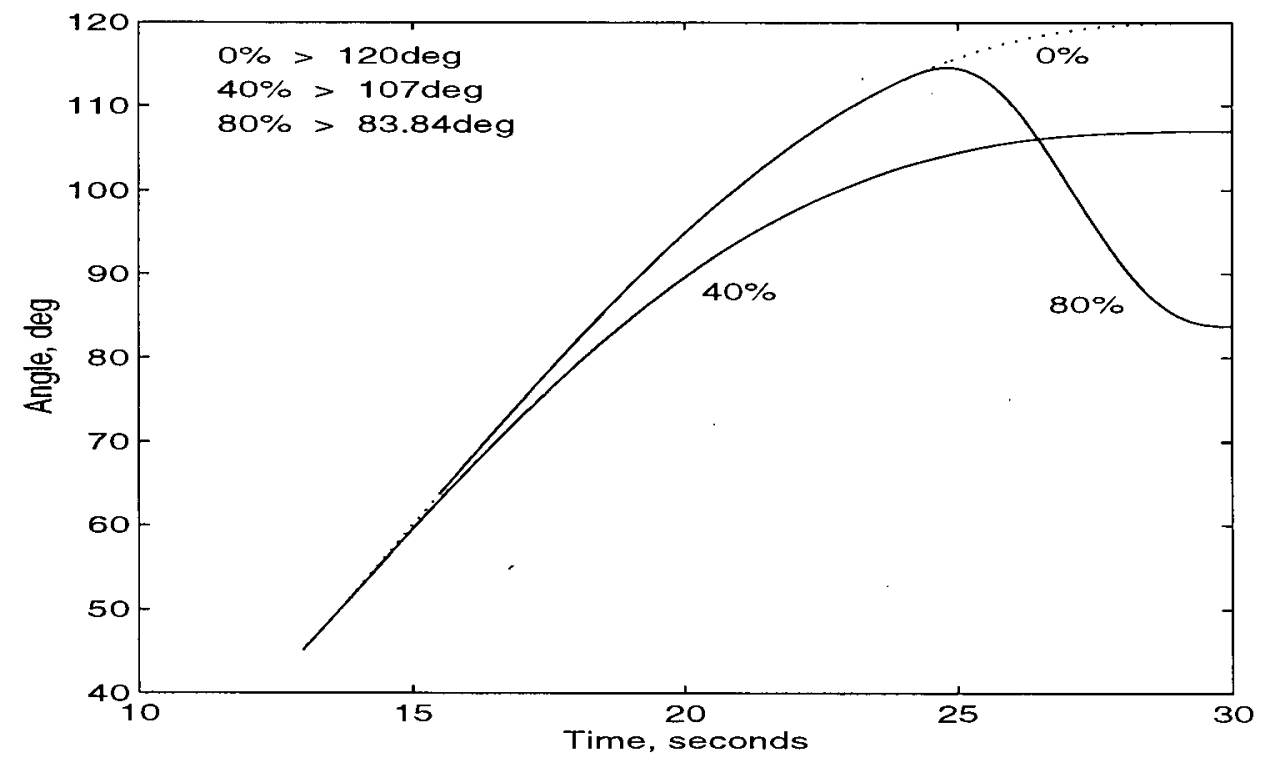

Figure 5.11: Single Update $\theta_{2}$ vs. Time 


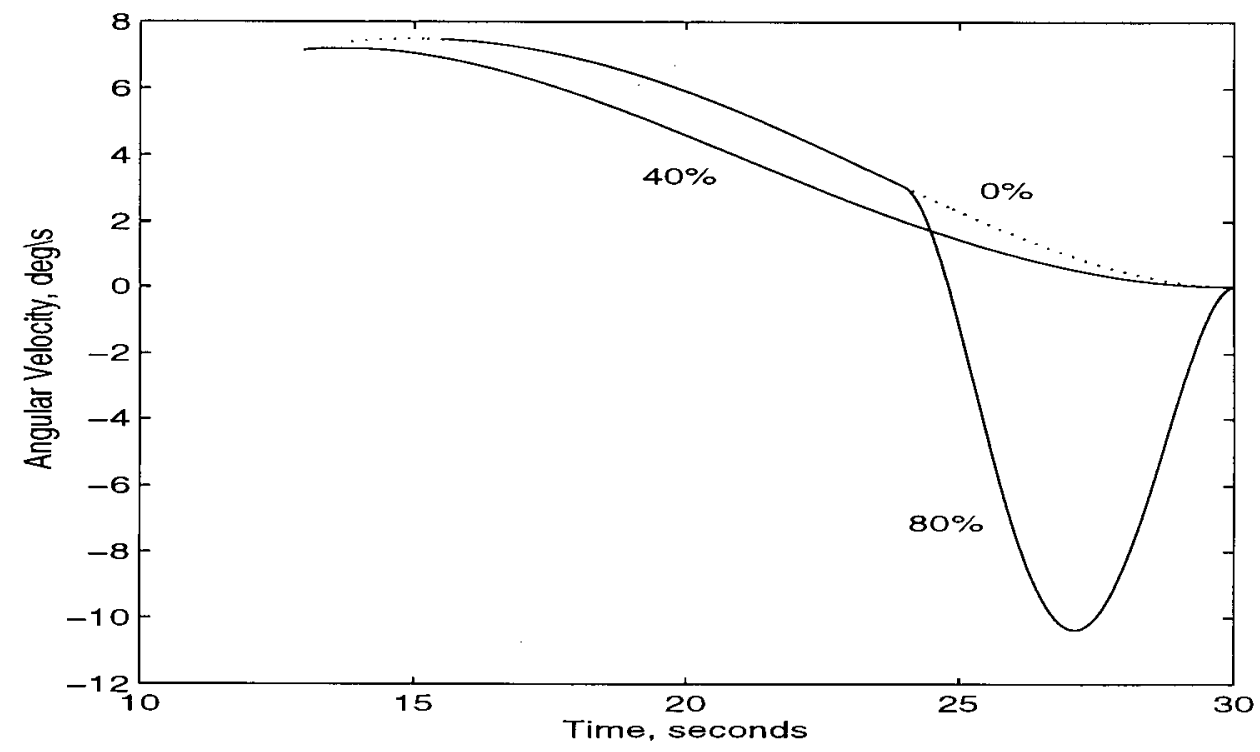

Figure 5.12: Single Update $\dot{\theta}_{2}$ vs. Time

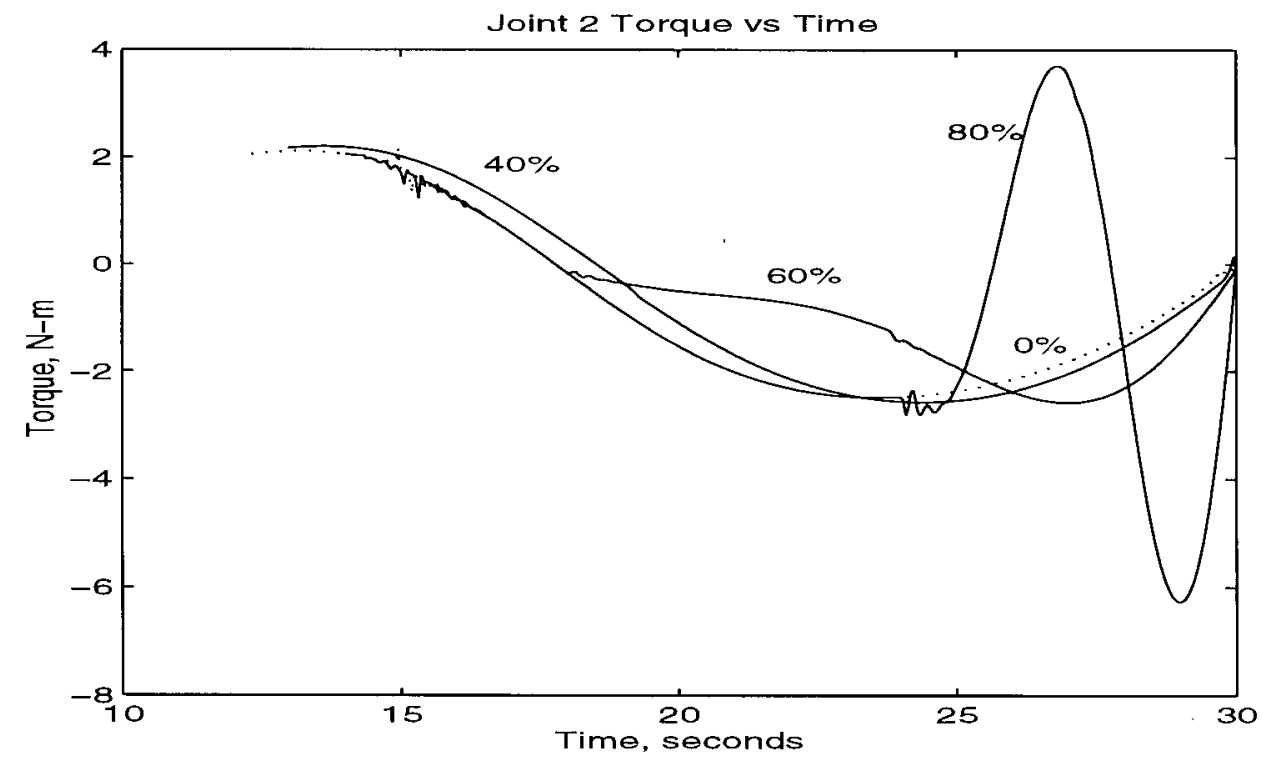

Figure 5.13: Single Update Joint 2 Torque vs. Time 

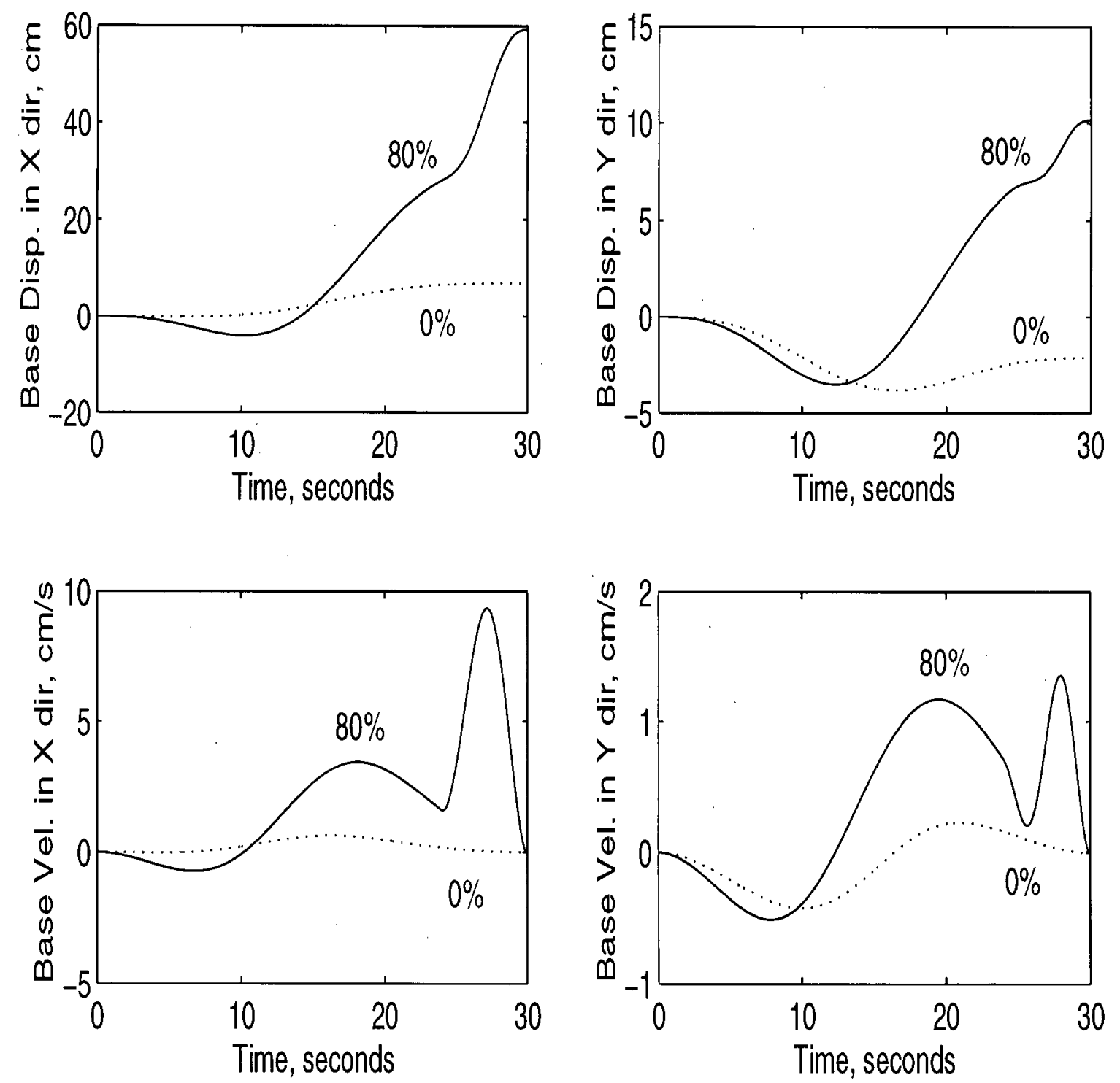

Figure 5.14: Single Update Base Position and Velocity vs. Time 

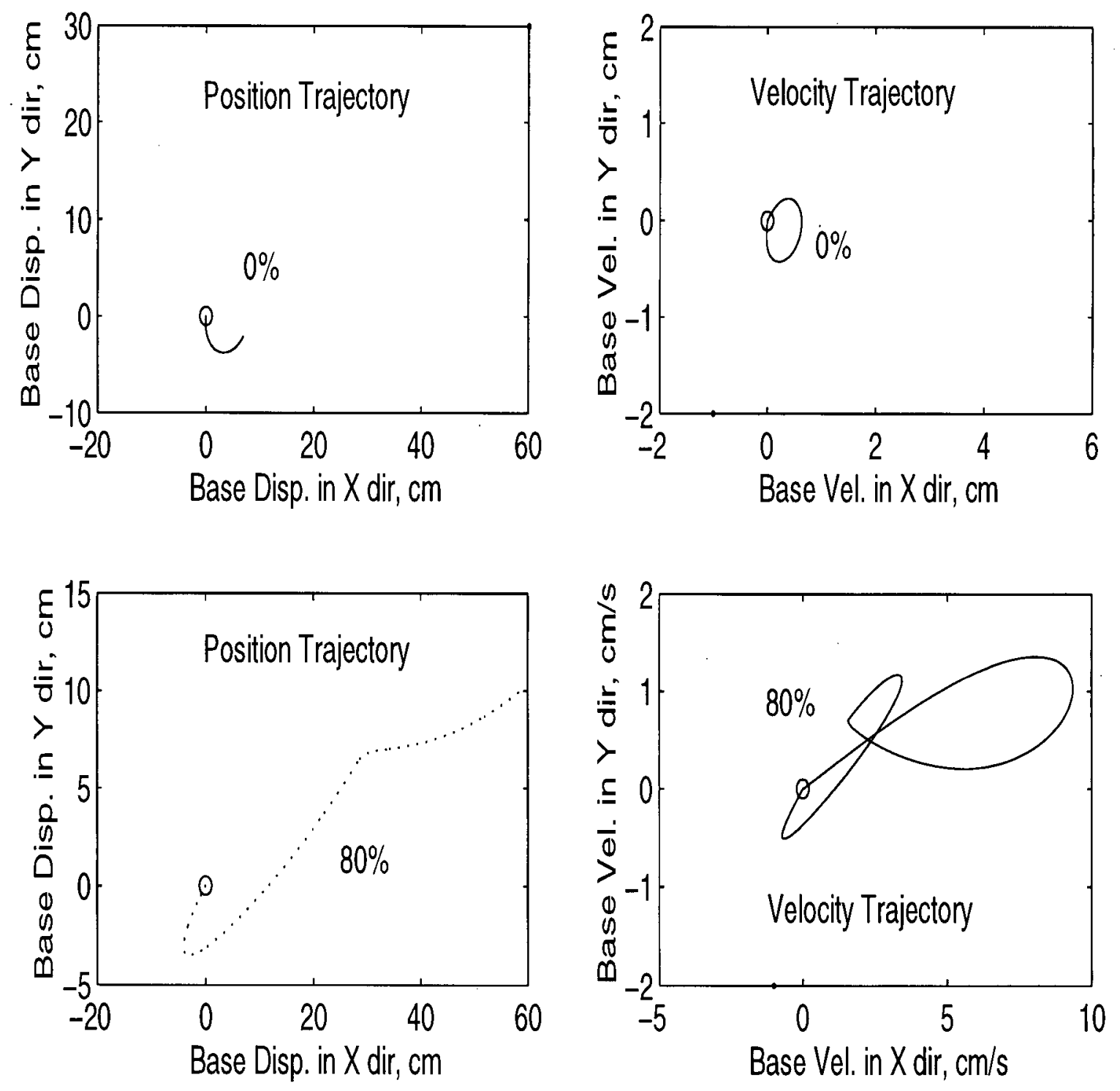

Figure 5.15: Single Update Base Position and Velocity 


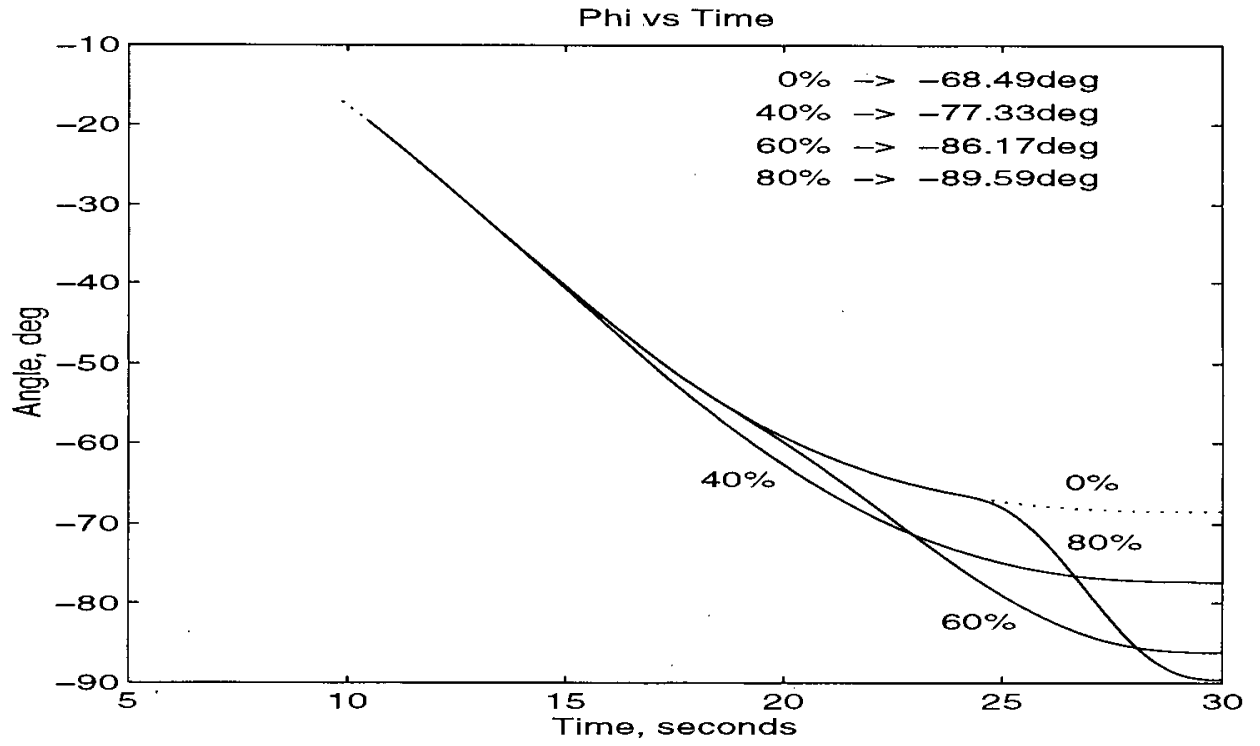

Figure 5.16: Single Update $\phi$ vs. Time

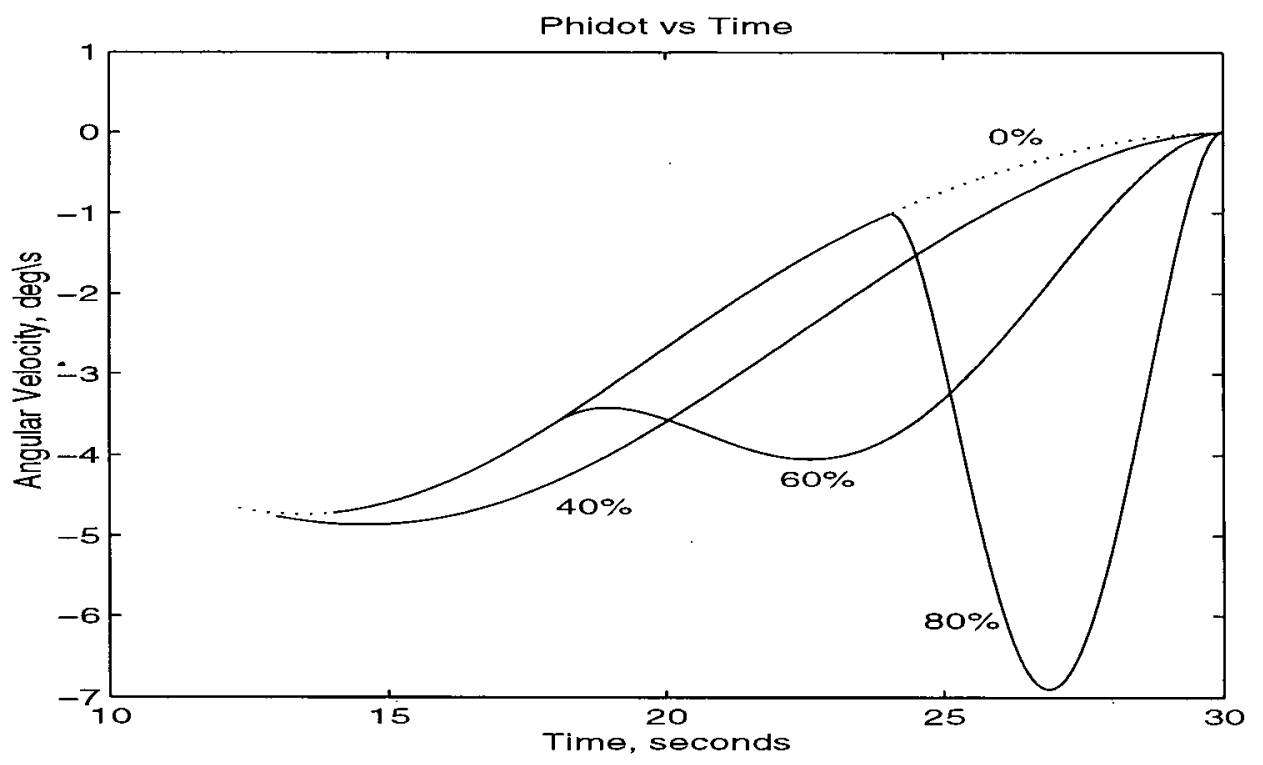

Figure 5.17: Single Update $\dot{\phi}$ vs. Time 
performed to produce this plot. The general trend for each curve is decreasing, confirming that updating later in the movement is better. But because the curves flatten out, the improvement is minimal after approximately $70 \%$ The reason for the very large errors of the top two curves is that the desired point moves out of the robots workspace and therefore becomes unreachable (unless the system is moved by thrusters). The other observation is that the farther the desired end-effector position is from the original position, the larger the overall error. This is only a generalization and can be seen from the two bottom curves. Although there is a significant initial error (end-effector travel distance), the final error is approximately the same. The other thing to note is that performing only one update during a movement generally will not give acceptable end-effector accuracy results, unless $R_{M}$ is very large. Therefore, multiple updates throughout the movement will have to be performed and are discussed in the next section.

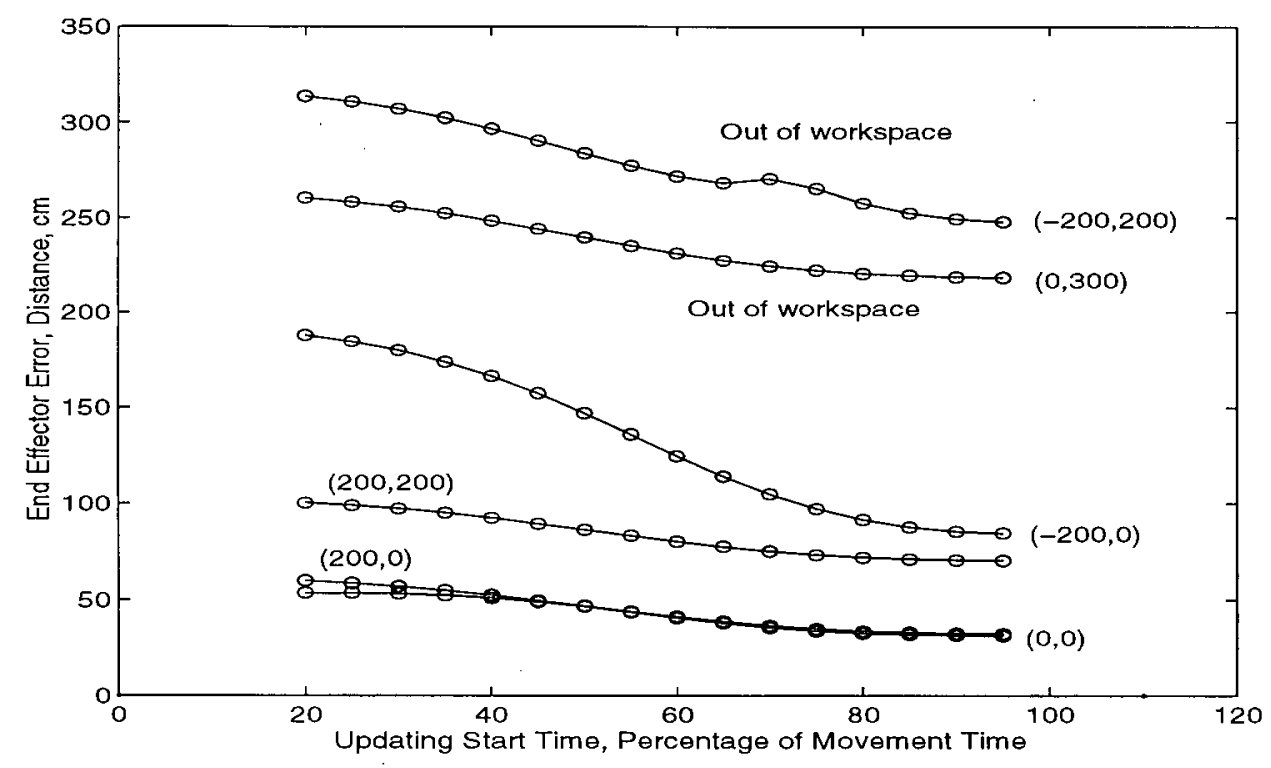

Figure 5.18: Single Update End-Effector Error 


\subsection{Effect of Multiple Updates and Update Frequency}

If updating is done throughout a movement the variables in question are when to begin updating and how frequently should updates be performed. A large number of simulations were done and several observations were made. One observation was that early updating yielded better results. Another observation was that frequent updating was better than infrequent updating. Figure 5.19 shows the system for a movement with and without multiple updating, the frequency being $2.5 \%$ of total time starting at $80 \%$ (two updates). Again, without an update, the robot achieves its commanded position in the robot frame but in the inertial frame misses the desired point by approximately $190 \mathrm{~cm}$. If updating begins at $80 \%$ of the total movement time, the error is reduced to $12 \mathrm{~cm}$ as opposed to $100 \mathrm{~cm}$ for a single update at $80 \%$. Clearly there is a huge improvement in even two updates (over one) if performed near the end of the movement. Figure 5.20 shows end-effector trajectories of movements at $80 \%, 60 \%$, and $0 \%$ (no update). It is observed here that when updating was started at $60 \%$ of the movement the end-effector error dropped to $23 \mathrm{~cm}$, in contrast to an error of $41 \mathrm{~cm}$ with multiple updates starting at $80 \%$. This shows that early updating results in less error. This is the opposite of single updates, where a later update is better. Figure 5.21 shows the joint angles for the different update times. In this plot at each update the final desired angle increases. Figure 5.22 and Figure 5.23 show the joint angular velocity and torque for joint 1 only. The earlier the updating the smaller the required velocities and torques (the opposite of single updates) and hence less base disturbance. This is because there is no big error correction at the last minute. Figure 5.24 shows the robot end-effector error in $\mathrm{cm}$ versus the update start time for multiple updates occuring at every $10 \%$ after the first update. As in the single update section, each curve in the plot represents a different desired position in the robot base frame, with the original end-effector position being $(400,0) \mathrm{cm}$. Six representative positions were chosen in the robot workspace. The circular points on the curves are the final error in $\mathrm{cm}$ for one 30 second movement. The general trend for each curve is increasing, confirming that beginning updating earlier in the movement is 


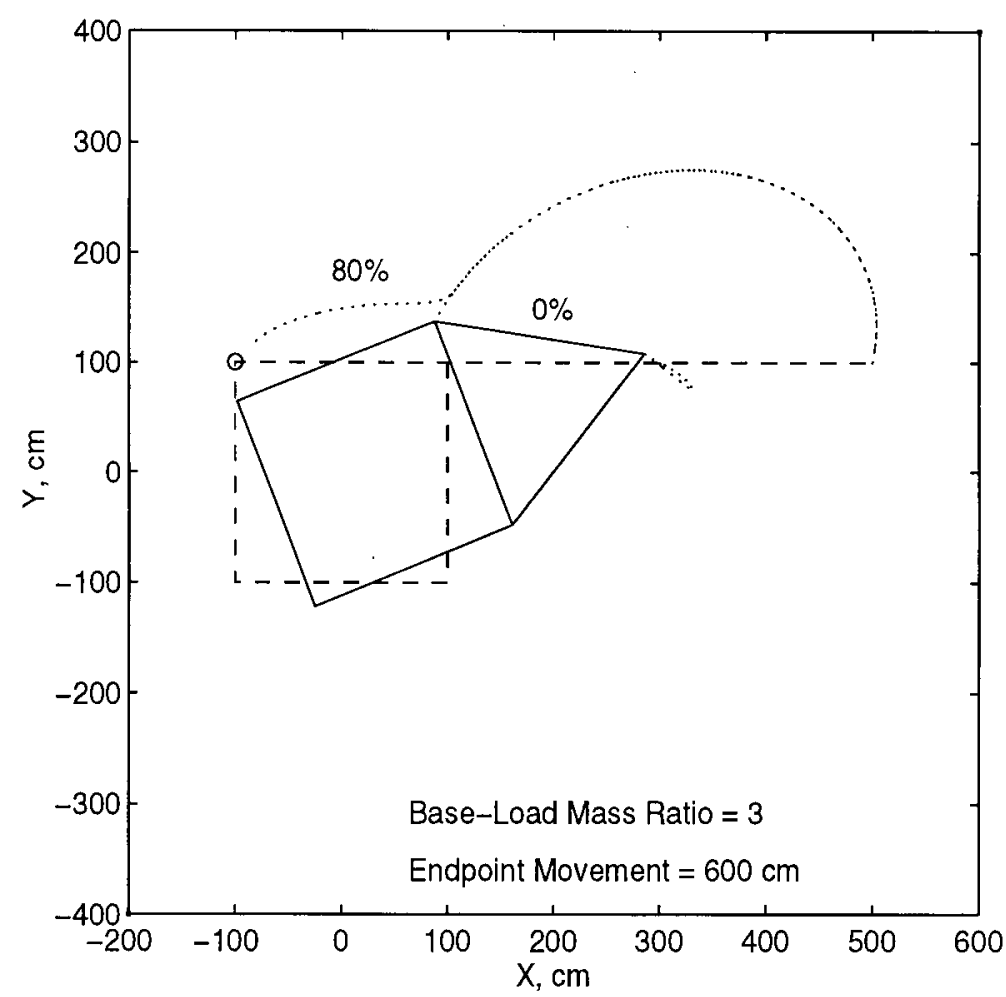

Figure 5.19: Multiple Update End-Effector Trajectories

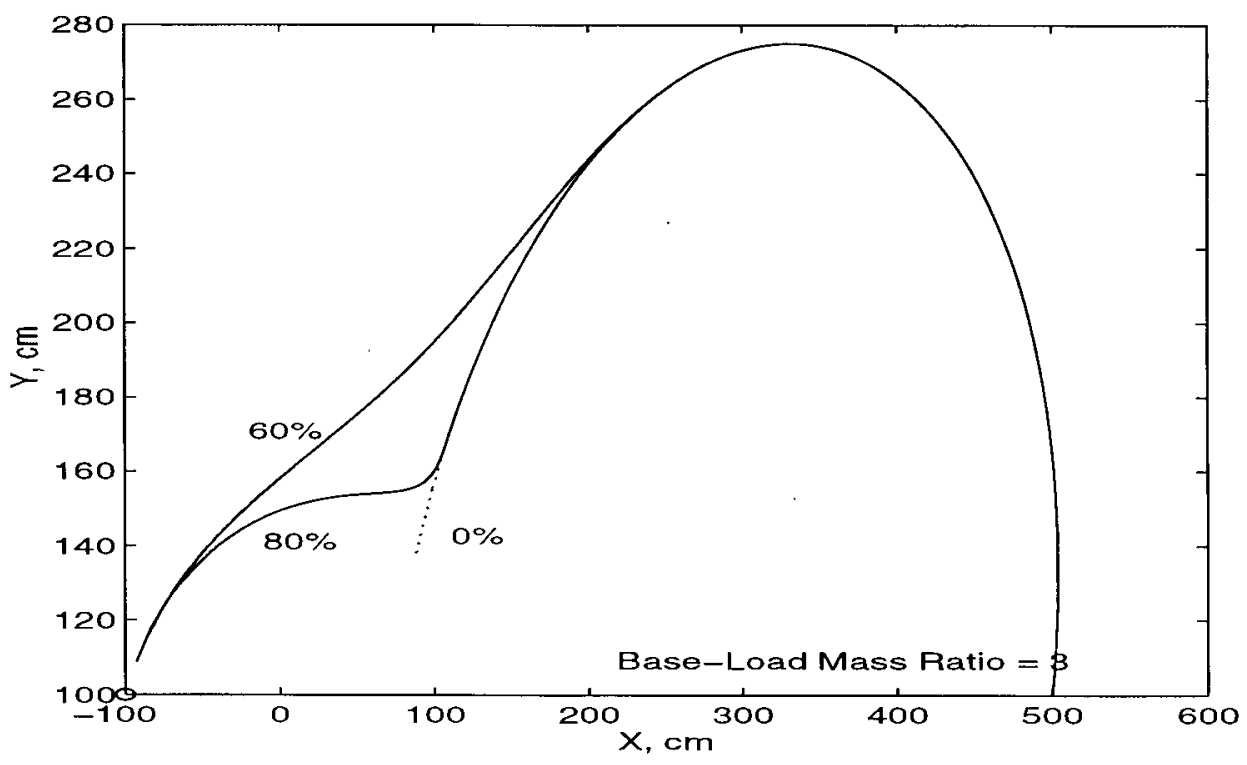

Figure 5.20: Multiple Update End-Effector Trajectories Close Up 


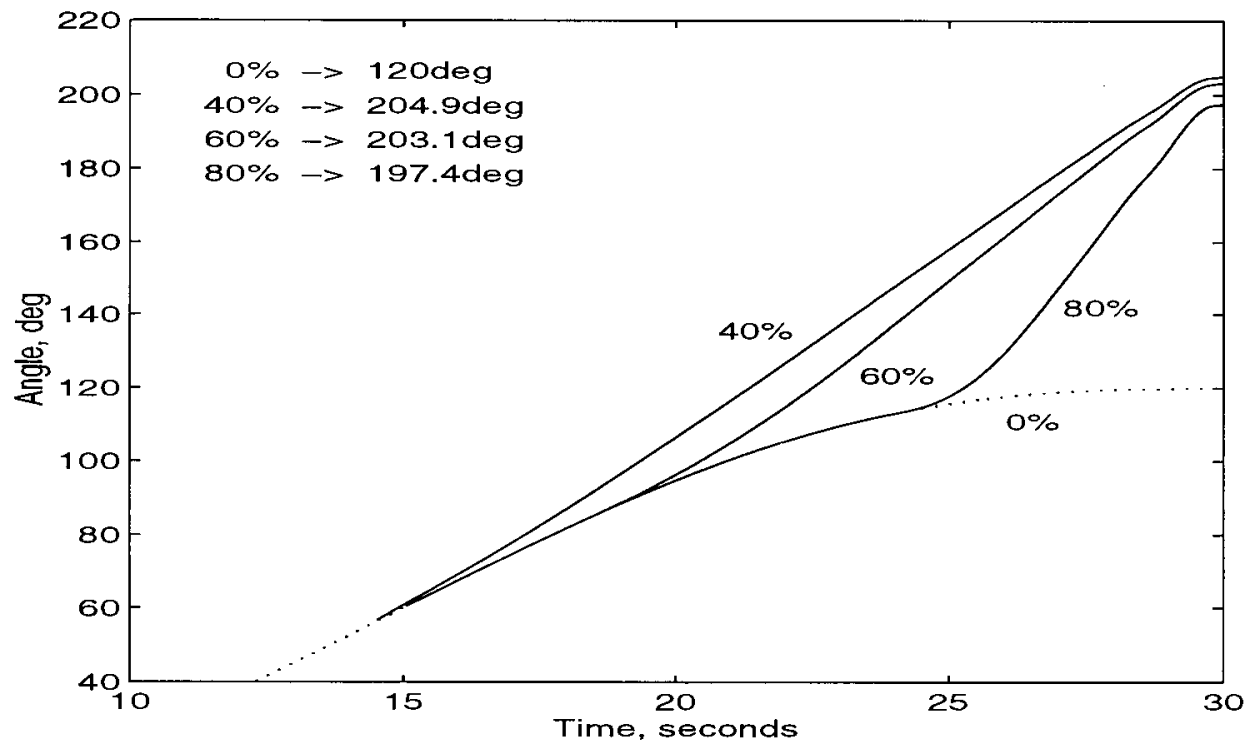

Figure 5.21: Multiple Update $\theta_{1}$ vs. Time

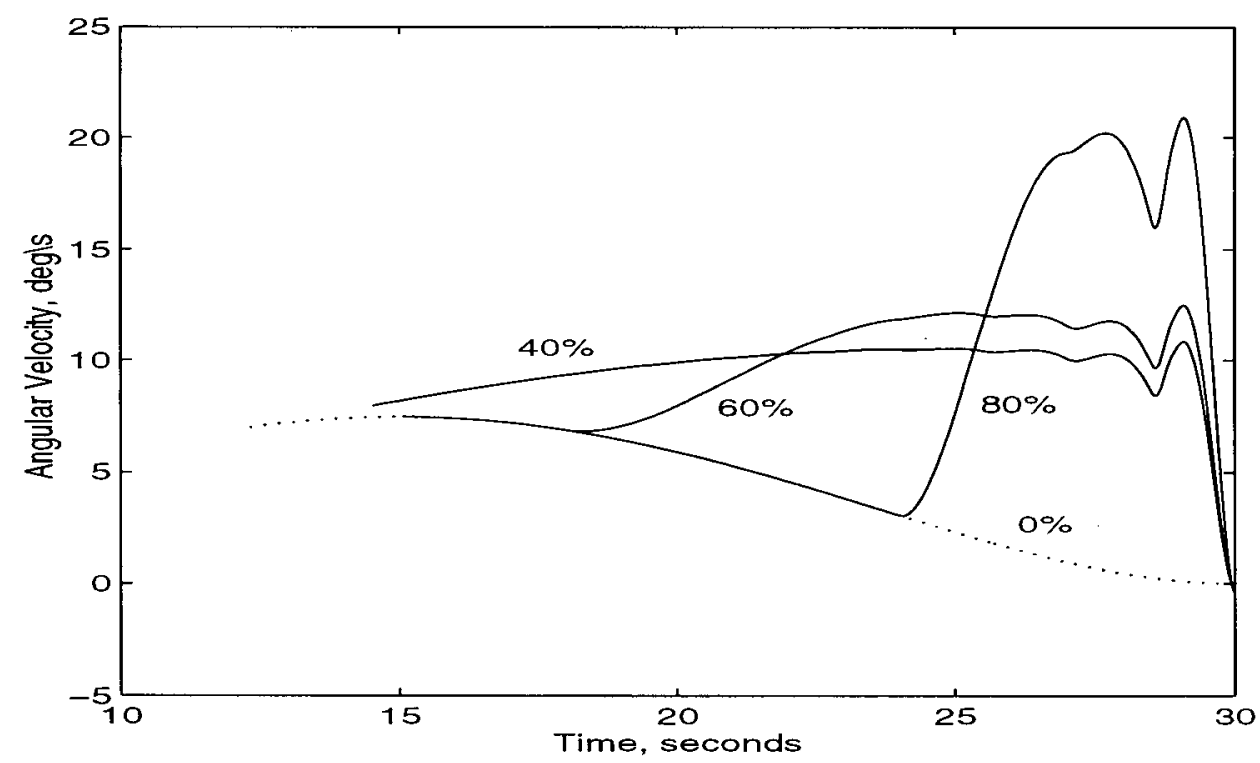

Figure 5.22: Multiple Update $\dot{\theta}_{1}$ vs. Time 


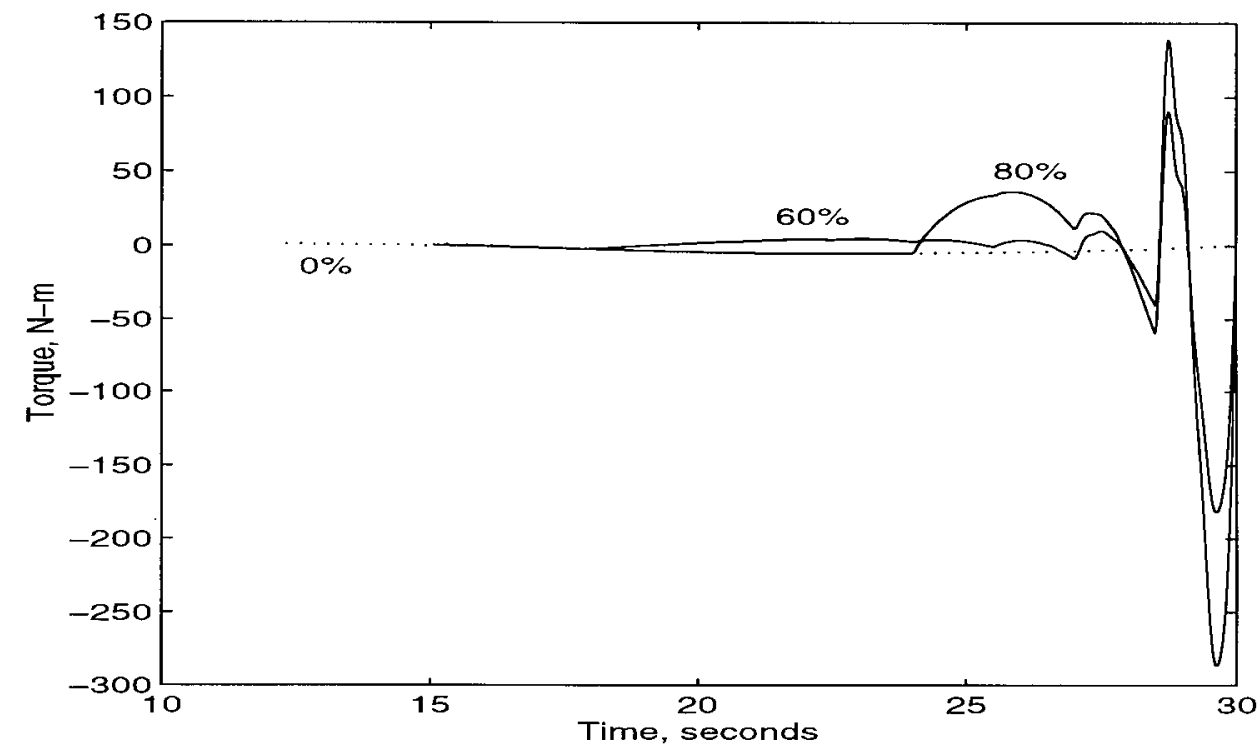

Figure 5.23: Multiple Update Joint 1 Torque vs. Time

better. As in the single update case, the reason for the large errors of the top two curves is that the desired point moves out of the robots workspace and is unreachable. One can note that the average error is now only about $175 \mathrm{~cm}$ as opposed to $230 \mathrm{~cm}$ for the single update case. Thus multiple updating gives a great improvement in manipulator reachable workspace and therefore less error. Figure 5.24 and Figure 5.25 shows the robot end-effector error in $\mathrm{cm}$ versus the update start time for multiple updates every for $5 \%$ and $2.5 \%$ of the movement time respectively. The most important observation inferred from these plots is that there is an optimum time to start updating. Since all the curves are relatively flat between 20 and $60 \%$, updating any earlier than $60 \%$ results in very little change in the final end-effector error. And because the curves $(2.5,5$, or $10 \%)$ begin increasing sharply after about $75 \%$, it is now possible to conclude that the optimum time to begin updating is approximately between 50 and $65 \%$ of the total movement time depending on the distance between the desired and initial end-effector positions. This can be thought of as when the robot begins closing in on the desired position. Performing multiple updating may give acceptable end-effector accuracy results depending on the requirements. An example of this is shown in the next plots for a desired position of $(0,0)$ 


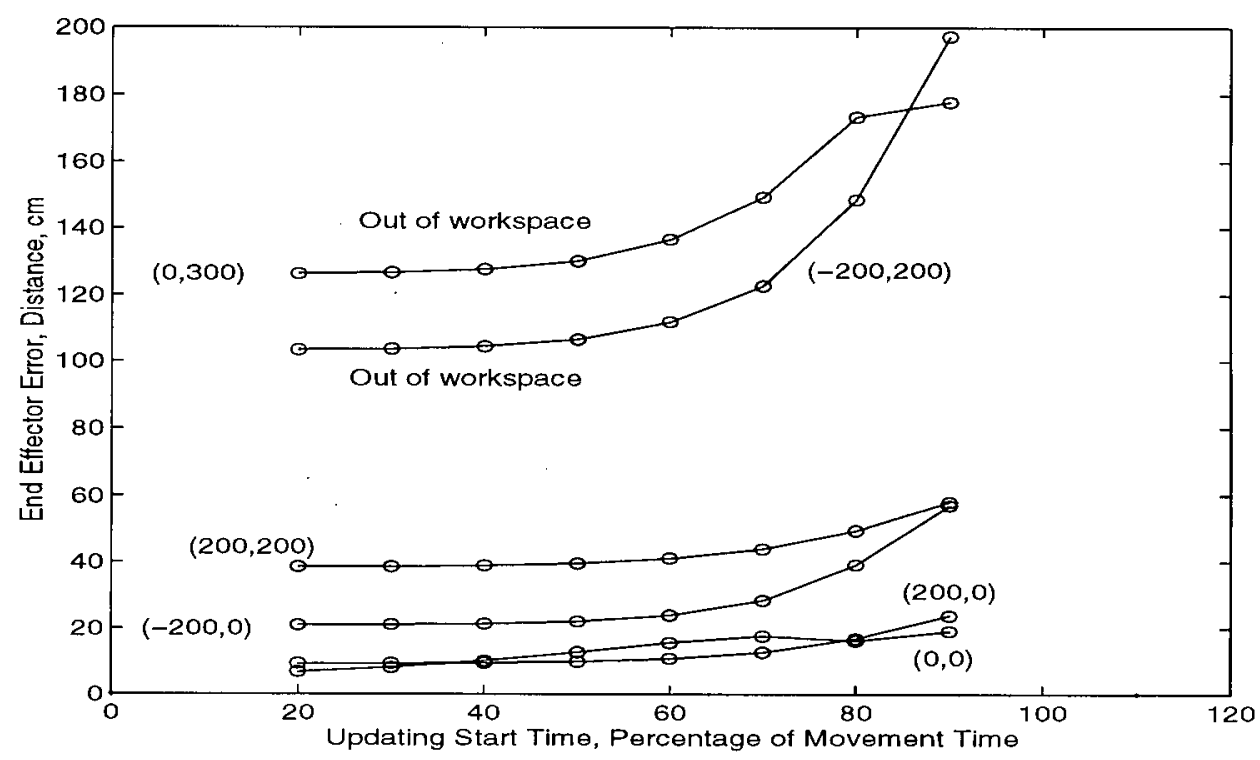

Figure 5.24: Multiple Update End-Effector Error - 10\% Steps

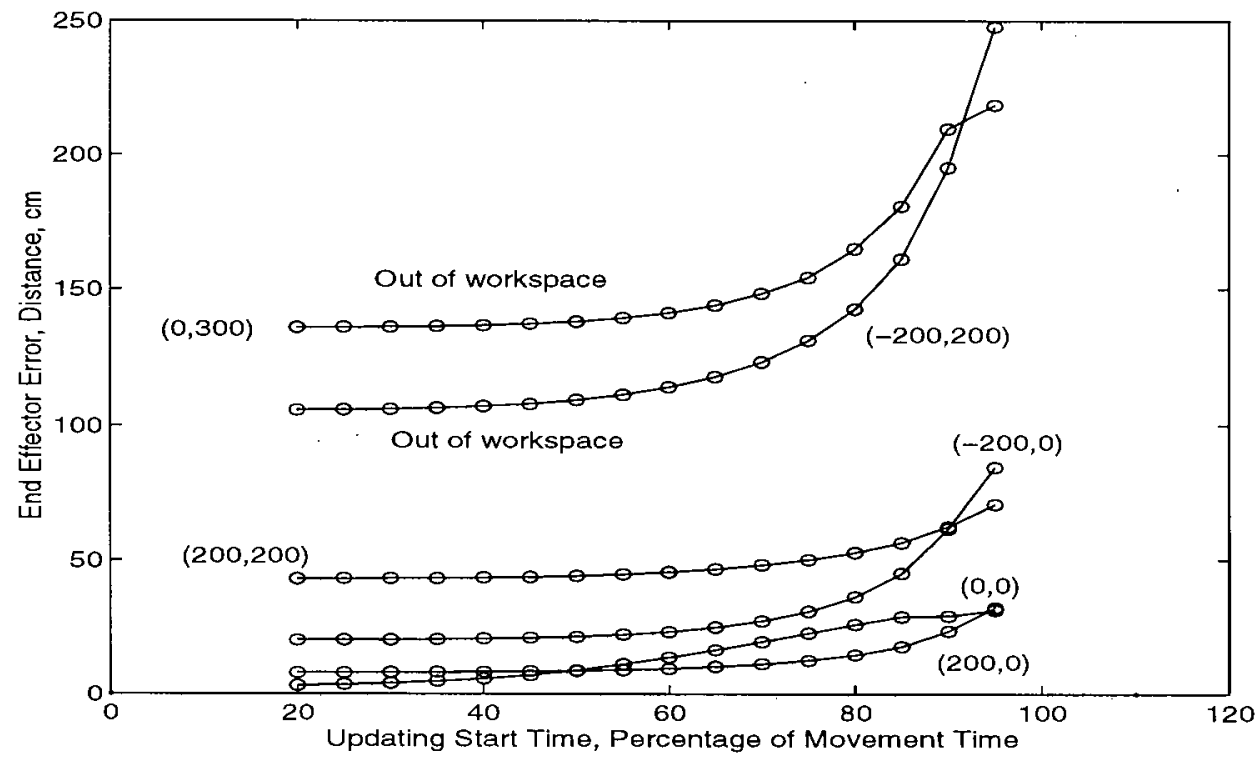

Figure 5.25: Multiple Update End-Effector Error - 5\% Steps 


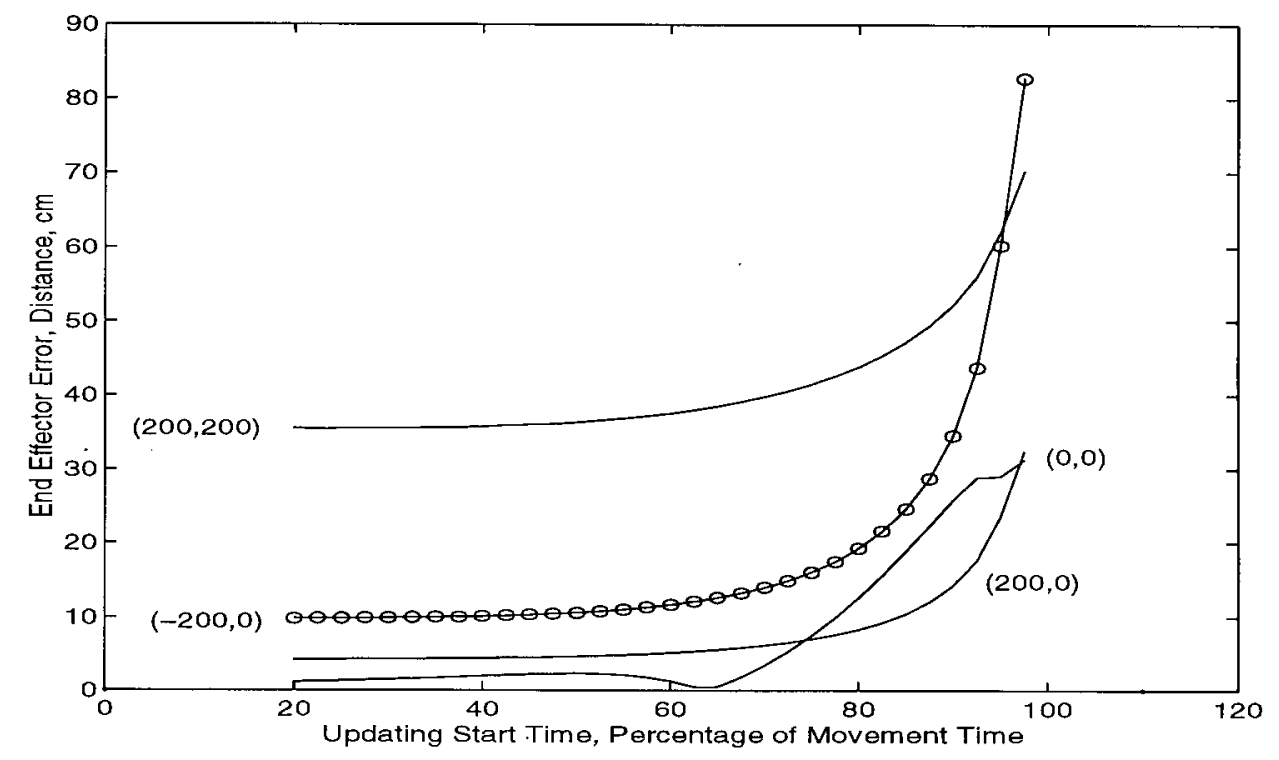

Figure 5.26: Multiple Update End-Effector Error - 2.5\% Steps

$\mathrm{cm}$. Figure 5.27 shows the end-effector error for $10 \%, 5 \%$, and $2.5 \%$ up frequency updating on a single plot. If the desired accuracy is less than say $2 \mathrm{~cm}$, beginning updating at $65 \%$ with a $2.5 \%$ frequency will obtain the desired accuracy. Figure 5.28 shows the system movement and Figure 5.29 shows the joint angle and angular velocities. For some desired points however using frequent updating starting at an optimum time still may not yield sufficient accuracy and therefore one of the methods (endpoint overcompensation and/or endpoint servoing) discussed in the next two sections will have to be incorporated.

\subsection{Effect of Endpoint Overcompensation}

If the accuracy obtained by performing an optimum set of multiple updates during a movement is not high enough, endpoint overcompensation $\left(E_{o c}\right)$ can be performed during the last part of the movement. In this method the desired end-effector position is moved slightly beyond the original one for the inverse kinematics recalculation. This is done linearly, such that if the desired position was $(0,0)$ and the end-effector was at $(1,1)$, if the update occurred at this point, 


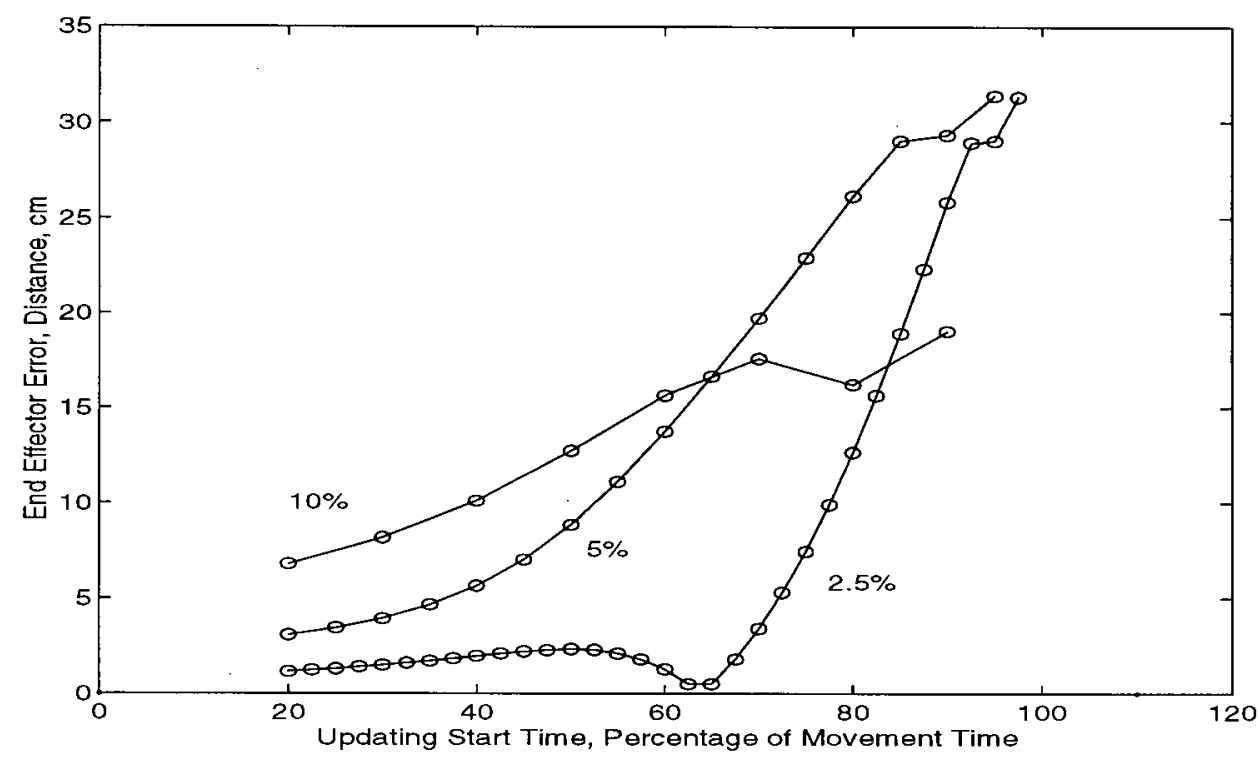

Figure 5.27: Multiple Update End-Effector Errors - $\mathrm{x}=0, \mathrm{y}=0$

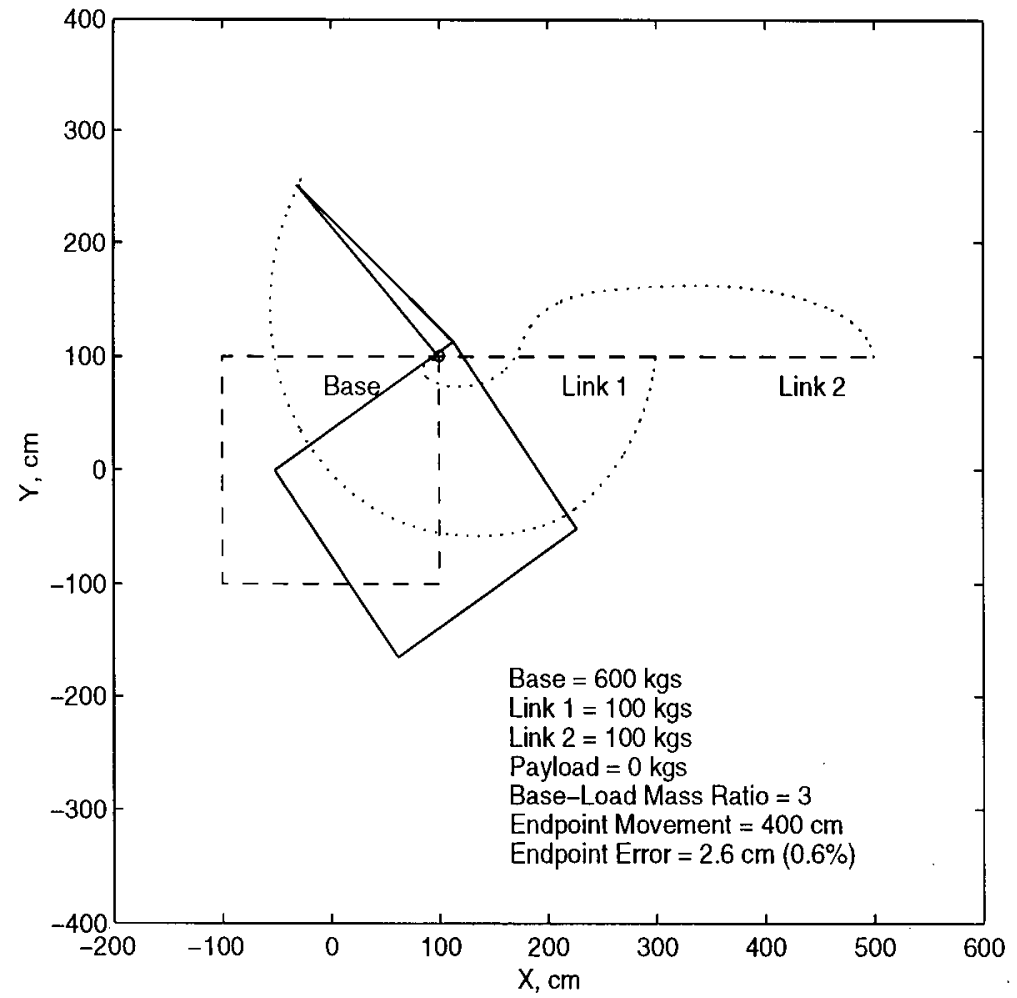

Figure 5.28: Multiple Update End-Effector Trajectory - $x=0, y=0$ 

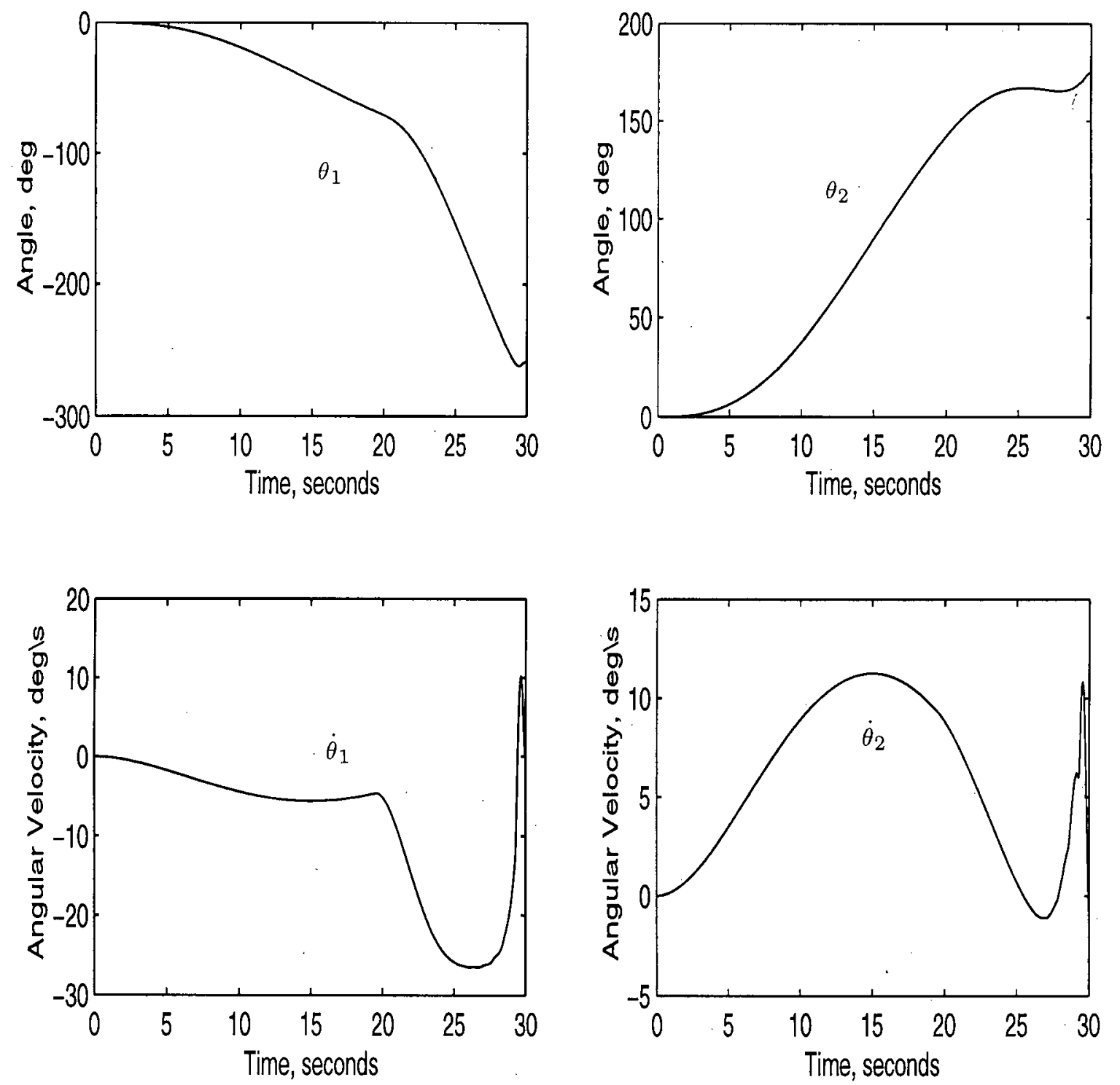

Figure 5.29: Multiple Update Angle and Angular Velocity - $\mathrm{x}=0, \mathrm{y}=0$ 
the algorithm would attempt to servo to $(-1,-1)$ (or some smaller distance in the direction of $(-1,-1))$. This could be done for each update or only for the last couple of updates. Figure 5.30 shows the overall system movement for a movement with and without $E E_{o c}$. Endpoint error is $42.6 \mathrm{~cm}$ without $E E_{o c}$ and $8.7 \mathrm{~cm}$ with $E E_{o c}$. If further accuracy is required after this step once the manipulator is stationary, then endpoint servoing as discussed in the next section is performed. It should be noted that endpoint overcompensation appears to improve performance in only a few configurations, particularly when the desired point is almost but not quite out of the manipulator's workspace.

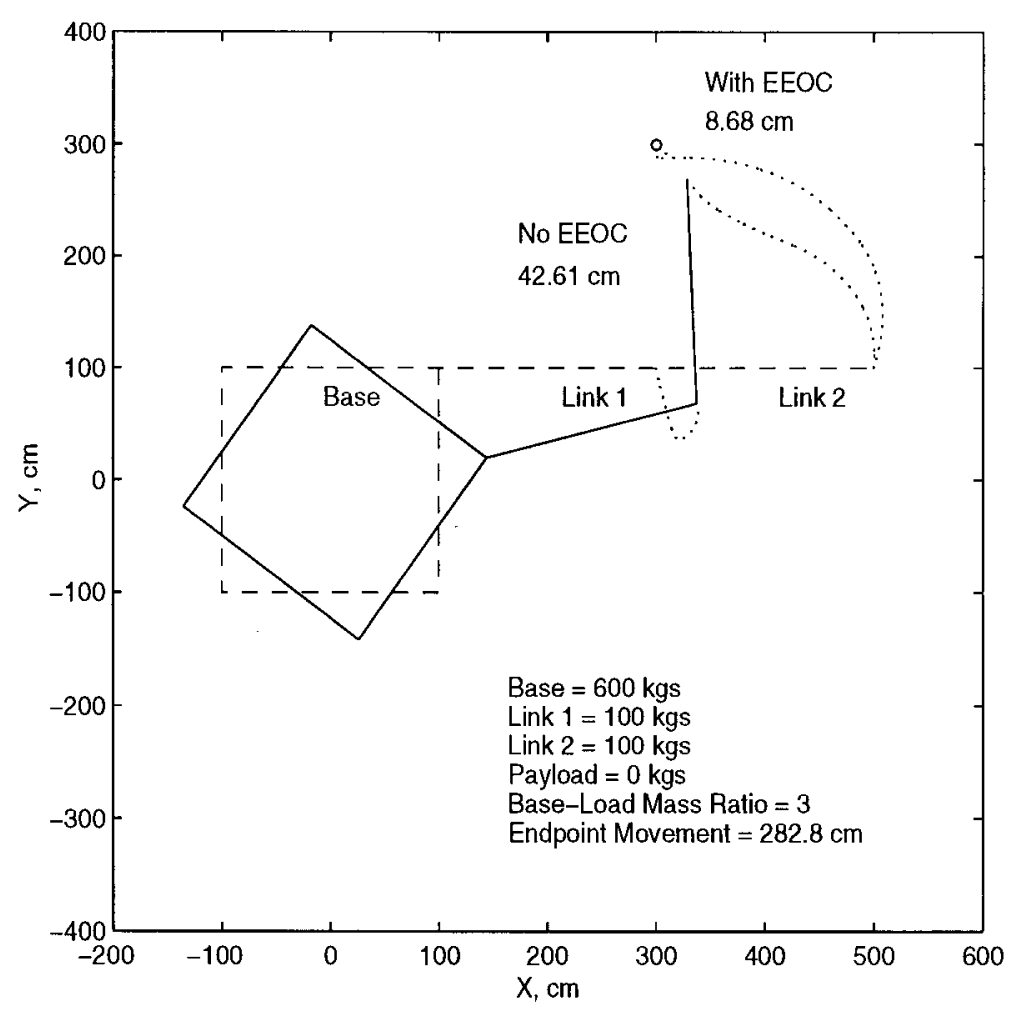

Figure 5.30: Endpoint Overcompensation - System Movement 


\subsection{Effect of Endpoint Servoing}

If further accuracy is required after multiple updates and possibly endpoint overcompensation, then one or more smaller manipulator movements are performed and are referred to as endpoint servoing $\left(E P_{\text {servo }}\right)$. At this point the manipulator end-effector will be relatively close to the desired position. With the system now at rest, another movement will be performed not unlike the one that previously occurred in that multiple updating is performed. But this movement will take less time to complete than the first large movement, and the end-effector can be positioned where desired with as high as precision as required. If very close positioning is necessary, then multiple movements may be required. It should be noted that since the manipulator is already close to the desired point thereby requiring small joint angular movements, there will be much less base disturbance than with the first large movement. Figure 5.31 shows the overall system movement for a movement with $E P_{\text {servo. }}$. In this simulation, two endpoint servos were done and very good results were obtained. In Figure 5.32 a close-up of the servoing trajectory is shown. Endpoint error is $24.2 \mathrm{~cm}$ without $E P_{\text {servo }}$ as seen marked in the bottom right corner. This is the end of the 30 second multiple updating movement. After the first 6 second $E P_{\text {servo }}$, the error is now $6.3 \mathrm{~cm}$, and after the second 6 second servo, the error is reduced to $1.9 \mathrm{~cm}$ (total movement $412 \mathrm{~cm}$ ). The algorithm is setup so either a set number of $E P_{\text {servo }}$ will be performed or alternatively, a maximum allowed error can be specified and $E P_{\text {servo }}$ will continue until the error is less than this value. 


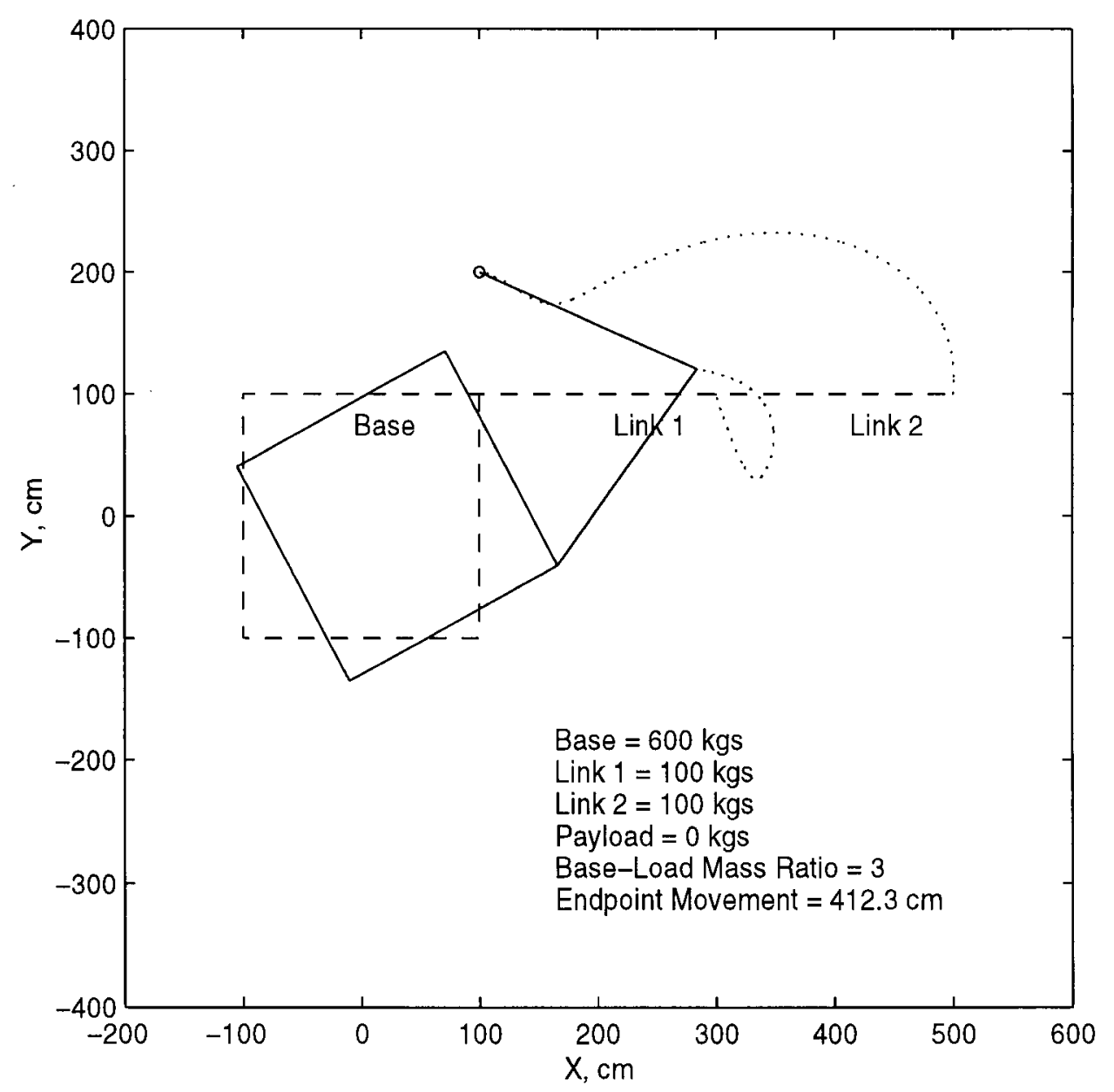

Figure 5.31: Endpoint Servoing - System Movement 


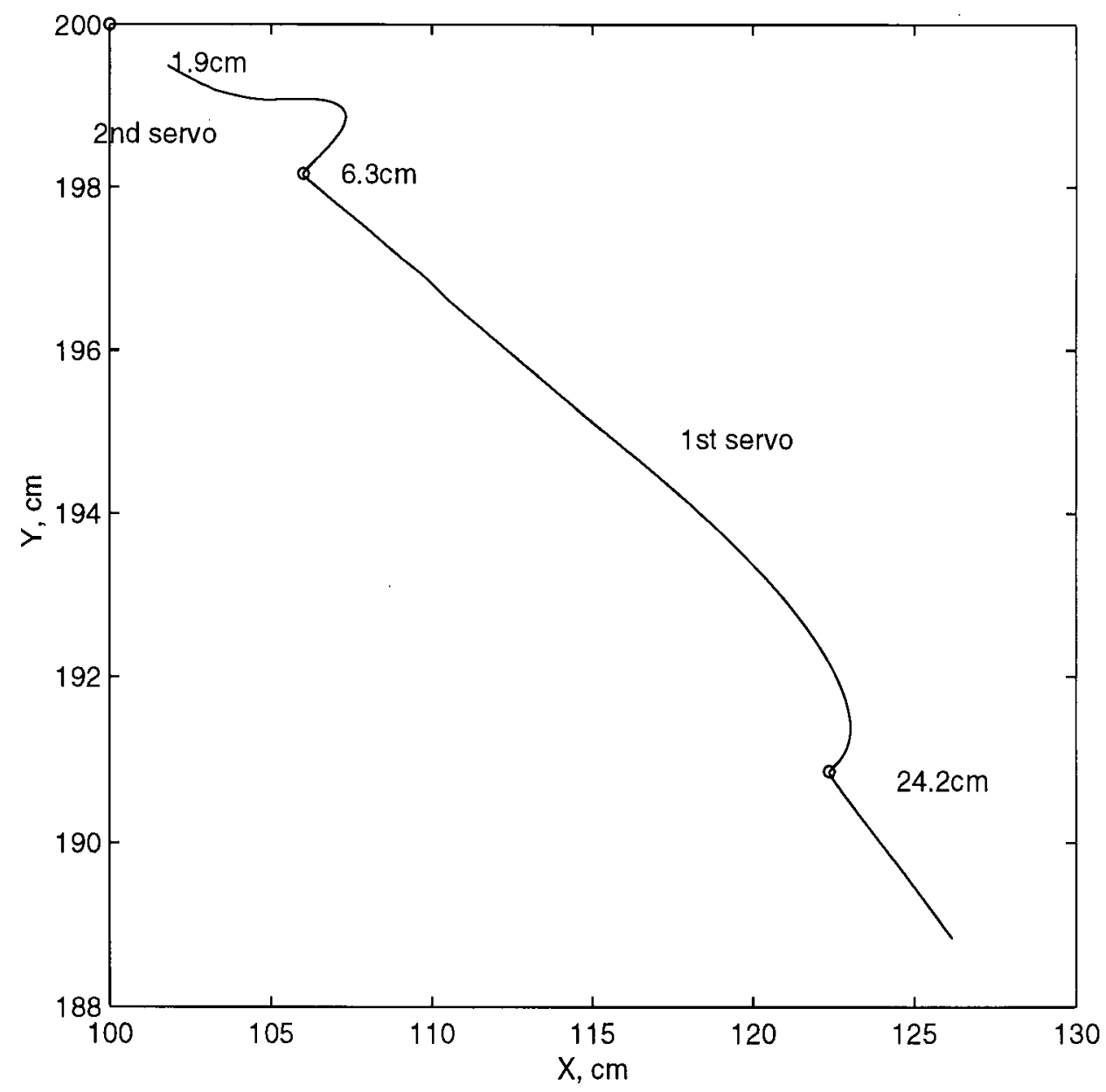

Figure 5.32: Endpoint Servoing - Trajectory Closeup 


\section{Chapter 6}

\section{Inverse Kinematics Guidelines and Performance Indices}

\subsection{Inverse Kinematics Updating Sequence Performance Indices}

To evaluate the performance for the results of a manipulator movement, certain criteria must be specified with which to judge the inverse kinematics updating algorithm performance. Some typical specifications that are listed when describing a (fixed base) robot are maximum payload, maximum joint and/or Cartesian speed, end-effector accuracy and repeatability, and Cartesian tracking error (adherence to a straight line). Specifications such as maximum joint velocity, payload, and repeatability are functions of the mechanical properties of the manipulator in question. Cartesian tracking error and accuracy (and repeatability) are dependent not only upon the mechanical properties of the robot but also on the performance of the control system. But because the manipulator is simulated and controller gains are chosen large enough to result in zero joint angular error, the manipulator is assumed perfect. Therefore manipulator endeffector accuracy in the inertial frame, as opposed to in the manipulator base frame, is used as a performance index in this thesis. Another performance index often used is the energy expended by the manipulator during a movement. This is a variable to be minimized or optimized when a movement is performed. Obviously performing manipulator - base positioning without the

use of thrusters or gyroscopes is pointless if more energy is consumed by the manipulator using an updating algorithm than with thrusters or gyroscopes. Energy consumed will not be used as a performance index, but it will be shown in the next section that performing multiple updates 
throughout a movement uses less energy than performing one movement without updating, then another without updating, etc.

Base disturbance or the robot base dynamic coupling factor is another criteria by which a particular manipulator movement can be related to system performance. The lower the base acceleration and movement the better the movement is judged to be. An extreme example would be a very large, fast movement with a heavy payload performed by the Canada Arm attached to Space Station Alpha. If this type of movement were possible and a large base disturbance resulted it would disturb and possibly be detrimental to people and equipment on board. Because of the nature of the on-line updating that this algorithm uses, the only way in which base disturbance can be minimized is by specifying low joint velocities and hence time scaling, which will result in minimal base disturbance. To actually use this as a variable, pre-movement off-line calculations that require all manipulator and base dynamic parameters are required. A good discussion and application of this is given in the paper "The Measure of Dynamic Coupling of Space Robot Systems", [Xu 93]. Here the author discusses the use of a performance index in planning robot motion, evaluating robot trajectories in minimizing base motion, and for optimizing the robot configuration design and robot base location on the base. The number of updates required to complete a movement with the desired accuracy can also be viewed as a type of performance index. Each time an update is performed there are inverse kinematics and possibly time scaling calculations to be done. These require CPU time and take some length of time to complete. Therefore the number of updates should be minimized, although accuracy is higher priority than update minimization. And for expended joint power minimization as discussed in the previous section, it was shown that more updates are preferred. Therefore the number of updates will be minimized if reducing the number has no effect on (primarily) accuracy and (secondarily) energy expended. 


\subsubsection{Manipulator Energy Expended}

It has been found that the more frequent the updates and the lower the joint velocities, the lower is the amount of actuator energy that is consumed. One possibility that is not investigated in this thesis but is recommended for future work is the concept of energy recovery. This could be implemented by using an electric generator for braking during the last part of a movement, rather than servoing the joints using the actuators the entire time. This is the same principle many electric cars now implement. Electricity is generated during braking which is then used for recharging the battery. To be used successfully in a manipulator application, research would have to done to determine the joint trajectories required. Actuator energy is determined using the following formula

$$
J_{\text {energy }}=\int \tau d \theta
$$

where $J_{\text {energy }}$ is the work or energy, $\tau$ is the joint torque, and $\theta$ is the joint angle. This equation is rewritten as

$$
J_{\text {energy }}=\int \tau \frac{d \theta}{d t} d t
$$

or

$$
J_{\text {energy }}=\int \tau(t) \dot{\theta}(t) d t
$$

and as a function of one expression

$$
J_{\text {energy }}=\int p(t) d t
$$

The functions in these expressions are the time trajectories of joint velocity and torque as seen in plots previously shown. The expression is numerically integrated in Matlab to give the energy consumed by the manipulator joint in Joules. The following example illustrates the benefits in terms of energy savings for a manipulator performing a single 30 second movement with multiple updating compared to three 10 second movements with no updating but servoing to the desired point at the start of each movement. In both cases the desired end-effector position is the same. Figure 6.1 shows the joint 1 torque and angular velocity for a single updating 
movement. In the third plot in the figure the product of the previous two curves, $P(t)$, is given. The energy expended by joint one is $5.3 \mathrm{~J}$ and for joint two is $1.4 \mathrm{~J}$ for a total of $7.8 \mathrm{~J}$. The end-effector error for this movement was $2 \mathrm{~cm}$ (total movement $412 \mathrm{~cm}$ ). In Figure 6.2 the torque, angular velocity, and $P(t)$ are seen for joint 1 for three no-update, 10 second movements. The expended energy for joints one and two are 5.0 and $25.6 \mathrm{~J}$ respectively. The total energy expended for these movements is 30.6 Joules, with a movement final error of $1 \mathrm{~cm}$. Clearly the updating algorithm used results in large energy savings as opposed to no updates due to overall lower torques and velocities required. A calculation was not done as to how much energy would be consumed by thrusters or gyroscopes if they were used to keep the base fixed for the same manipulator movement. This would require changes in the simulation model to include controllers for the base generalized coordinates. This means utilizing two thrusters for the base translational movement and a gyroscope for the rotational movement. The Runge-Kunta integrator for integrating the dynamic equations of motion would also need to changed.

\subsection{Guidelines for Inverse Kinematics Updating Sequences}

To determine the sequence of events that should occur during a movement, the desired results (i.e., accuracy) and the variables which accuracy depends on should be stated. As discussed in the last section, the main system performance index will be the inertial frame end-effector accuracy. Other performance indices that will be considered but not strictly adhered to are the number of updates, manipulator energy expended, and manipulator-base dynamic coupling or disturbance. The fixed system factors on which accuracy depends on are the base-arm mass ratio $\left(R_{M}\right)$ and the total endpoint movement. The total endpoint movement is also related to joint angular displacements. The algorithm factors that are varied to produce the required accuracy are the time that the update occurs, the frequency of the updates or the update sequence, endpoint overcompensation, and endpoint servoing. The secondary performance indices that can be minimized with no effect on accuracy are joint energy expended and dynamic coupling as 

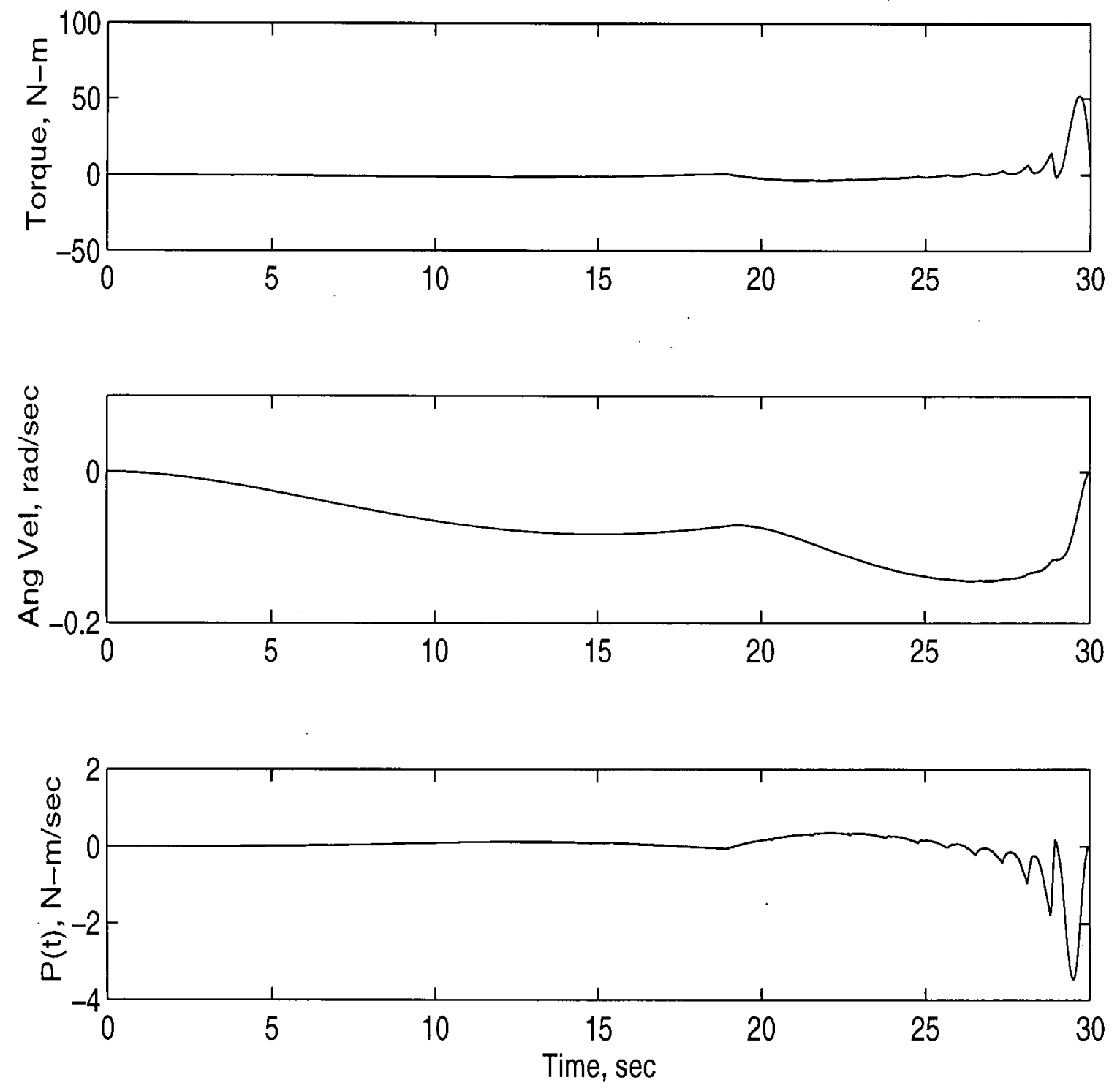

Figure 6.1: Joint Angular Velocity and Torque - Multiple Updating 

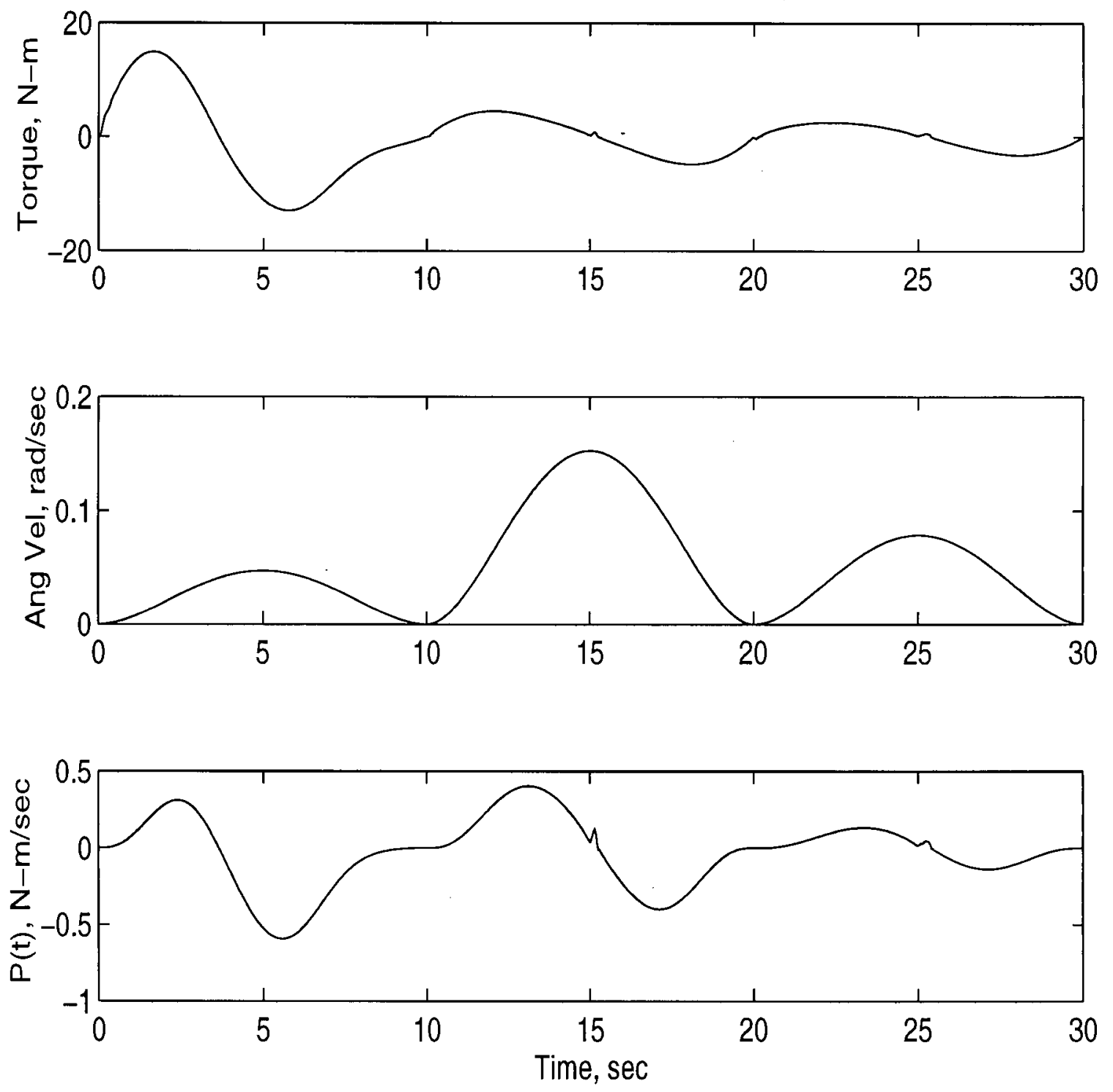

Figure 6.2: Joint Angular Velocity and Torque - 3 Single Movements 
these depend on total movement time. As discussed in the section on time scaling, the movement time can be lengthened to minimize the energy consumed and the base disturbance, and will have no effect on accuracy. To devise an algorithm that will produce the desired accuracy for any system initial configuration and properties, a sub-algorithm or equation is written that shows the dependencies of these factors. It will be used as a guideline for determining the updating sequence. First all the variables are assigned a notation and their dependencies shown in the general equation below

$$
\left(A C C_{e}, E N_{j}, N U M_{u}, D I S T_{b}\right)=f u n c\left(R_{M}, \Delta_{e}, T_{u}, S_{u}, E_{o c}, E P, T_{\text {total }}, V_{j}, \tau_{j}\right)
$$

where

- $A C C_{e}$ : Accuracy of the end-effector position in the inertial frame

- $E N_{j}$ : energy expended by a manipulator during a movement

- $N U M_{u}$ : number of updates

- $D I S T_{b}$ : base disturbance during movement

- $R_{M}$ : base-manipulator mass ratio

- $\Delta_{e}$ : total required end-effector movement distance

- $T_{u}$ : time during a manipulator movement when an update performed

- $S_{u}$ : Update sequence during a manipulator movement

- $E_{o c}:$ endpoint over compensation

- $E P$ : endpoint servoing at the end of a movement

- $T_{\text {total }}:$ total movement time

- $V_{j}$ : maximum joint velocities 
- $\tau_{j}:$ maximum joint torques

The variables that will be incorporated into the algorithm for general cases are shown in the equation below.

$$
\left(A C C_{e}\right)=\operatorname{func}\left(R_{M}, \Delta_{e}, T_{u}, S_{u}, E_{o c}, E P\right)
$$

Based on the results of the previous sections, we know that end-effector accuracy and therefore when the updating should start depends largely on the $R_{M}$. We write a general analytical equation of updating start time determined through experimentation as a function of $R_{M}$ as follows

$$
T_{u}=55+35\left(1-2^{-\frac{R_{M}}{15}}\right)
$$

A graph of this function is seen in Figure 6.3. The coefficients are varied so that the curve fits

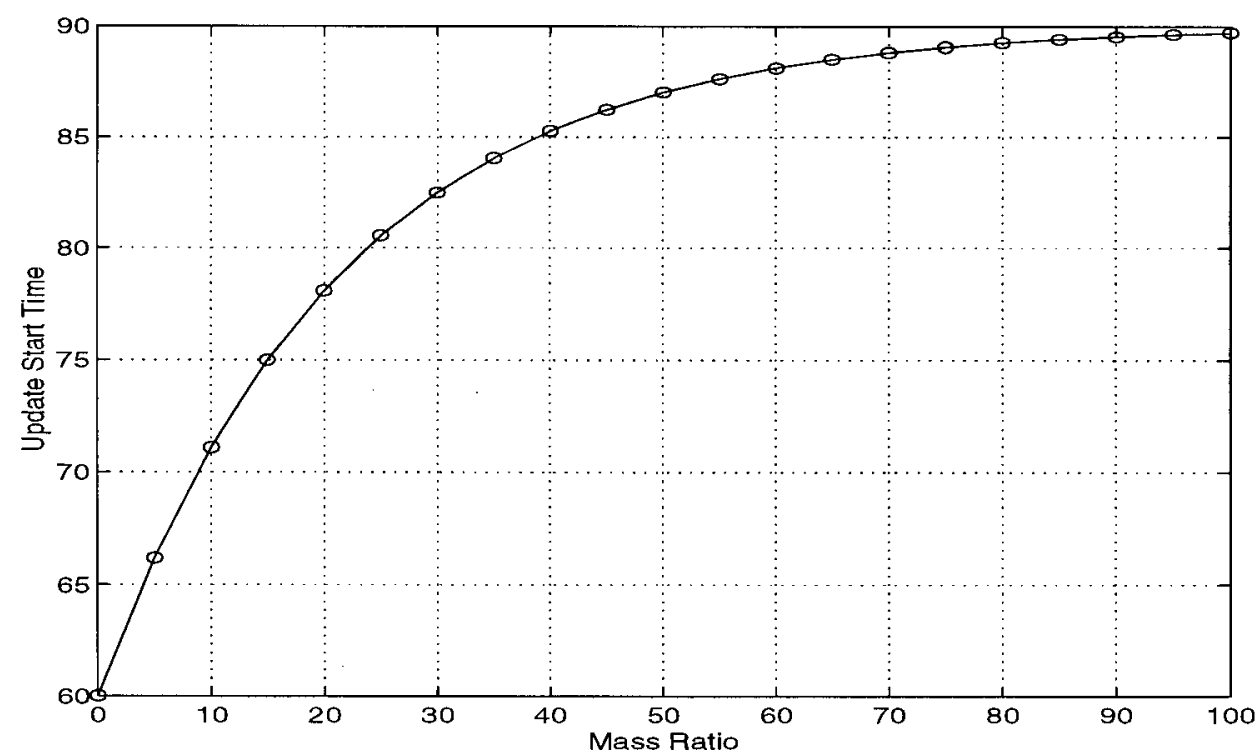

Figure 6.3: Update Start Time vs. Mass Ratio

the previously determined observations. Updating in any case should not begin sooner than $55 \%$ of the way through the movement and no later than approximately at $90 \%$ of the way 
through the movement. The factor of 15 in the decaying exponential component was chosen so that the update time would be most "sensitive" to the mass ratio for low mass ratios, and less sensitive for higher mass ratios (larger slope at smaller mass ratios). $T_{u}$ can also be related to $\Delta_{e}$ or total required end-effector distance knowing that regardless of the mass ratio, if the manipulator end-effector is to move only a small distance relative to the total manipulator length, then updating could start a little later than would be justified by Equation 6.7. If a very large distance is required (maximum 200\% or twice manipulator length) then updating should start sooner. Therefore $T_{u}$ will be multiplied by a factor related to the end-effector distance. This factor will vary between 0.8 and 1.2 depending on $\Delta_{e}$, the equation being a linear one and written as

$$
k_{T_{u}}=\left(1.2-0.002\left(\frac{\Delta_{e}}{200}\right)\right)
$$

This equation was derived to give the desired function slope. A graph of this function is seen in Figure 6.4. The new update time is then given by

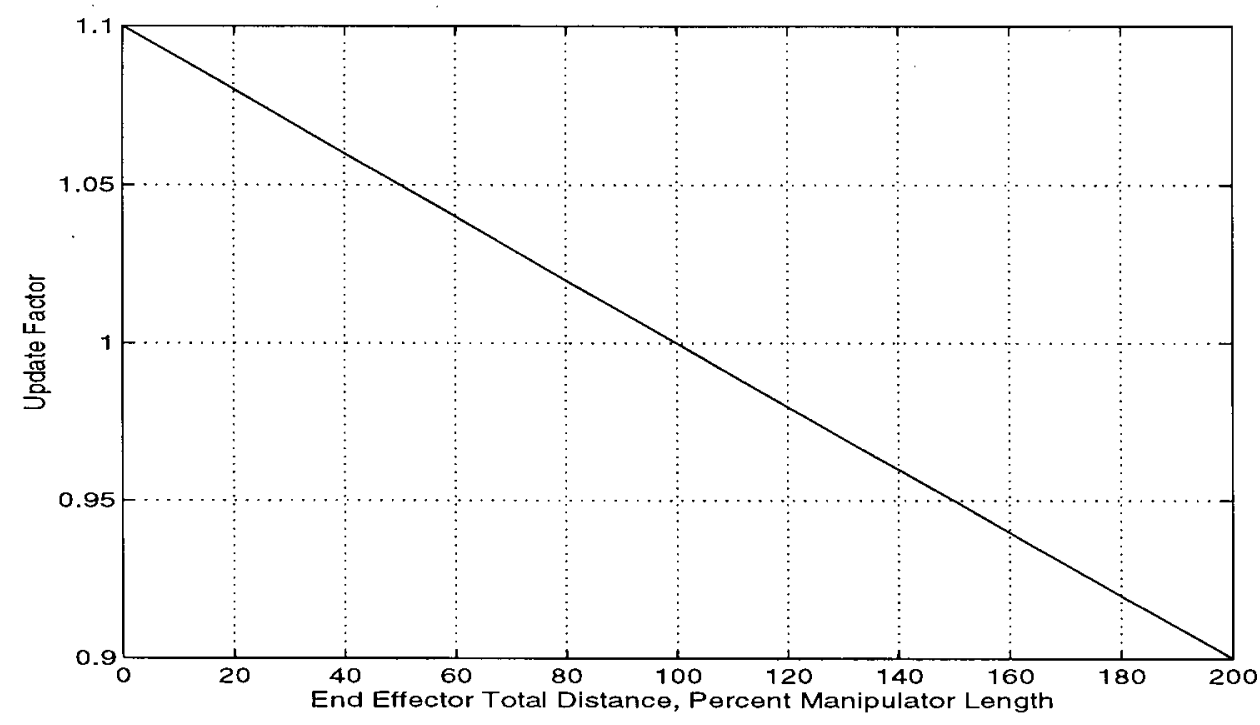

Figure 6.4: Update Factor vs. End-Effector Total Distance 


$$
T_{u}=k_{T_{u}} T_{u}
$$

It has been determined that the higher the update frequency the better the final accuracy. And to reduce the total number of updates required a function will be chosen so that the update frequency increases as the movement progresses. As a guideline for an updating sequence function, a decaying exponential curve is used similar to the one used to determine $T_{u}$. The sequence should begin approximately at $T_{u}$, and the latest an update should occur is approximately $96 \%$ of the total movement time. If time and velocity scaling are enabled an update can occur later and excessive torques and velocities won't result due to the increased movement time. The experimental function is written as

$$
S_{u}=T_{u}\left(2-2^{-\frac{\mathbf{k}_{\text {step }}-T_{u}}{\text { coef }}}\right)
$$

where coef is given as

$$
\text { coef }=25+\left(T_{u}-60\right)
$$

and $\mathbf{k}_{\text {step }}$ is a linear sequence used as a reference sequence starting at $T_{u}$ and increasing in steps to approximately the time of the last update or $T_{\text {updlast. }}$. A step size for this sequence is obtained using

$$
k_{\text {stepsize }}=\frac{T_{u}}{20}
$$

and is an average size of the actual update sequence interval. The number of steps $\left(N_{k}\right)$ performed is given by result of the following equation rounded up to the next integer value

$$
N_{k}=\frac{T_{u p d l a s t}-T_{u}}{k_{\text {stepsize }}}
$$

Because of the rounding up of the number of steps, if starting at $T_{u}$ and adding steps of size $k_{\text {stepsize }}$ to $T_{u} N_{k}$ times, the final update time will be slightly larger than $T_{\text {updlast }}$. Therefore the difference between these values is subtracted from each element in the updating sequence so that the step size is $k_{\text {stepsize }}$ and so that the last step occurs at $T_{\text {updlast }}$. The result is that $T_{u}$ is smaller so that updating begins a little sooner than the original value. The new $S_{u}$ sequence 
is calculated as shown below

$$
S_{u}=S_{u}-\left(S_{\text {updseqoriginal }}(\text { lastvalue })-T_{\text {updlast }}\right)
$$

A graph of this updating sequence is shown in Figure 6.5. Curves for sequences starting at different $T_{u}$ s are shown. As stated previously, the updates occur more frequently as the movement progresses. If further accuracy is required after this sequence, which may or may not incorporate endpoint overcompensation, then servoing is continued until the desired accuracy is achieved.

\subsection{Further Examples and Algorithm Verification}

In this section simulations to test the previous algorithms were performed with systems having different mass ratios, desired end-effector locations, and accuracy requirements. From these results an overall performance rating for the updating algorithm can be obtained. The results from a series of simulations with $R_{M}$ equal to 25 are shown in Table 6.3.1.

\begin{tabular}{|l|l|l|l|l|l|l|}
\hline \multicolumn{1}{|c|}{} & \multicolumn{7}{|c|}{ X desired position, cm } \\
\cline { 2 - 7 } & 200 & $6.6: 0.5$ & $5.7: 0.5$ & $5.5: 0.5$ & $4.9: 0.5$ & $4.6: 0.5$ \\
\cline { 2 - 7 } Y desired \\
\cline { 2 - 7 } position, cm & 100 & $2.4: 0.1$ & $2.2: 0$ & $2.8: 0.3$ & $0.9: 0$ & $0.3: 0$ \\
\cline { 2 - 7 } & 0 & 0.8 & 0.4 & 0.9 & 0.3 & 0.9 \\
\cline { 2 - 7 } & -100 & 0.1 & 0.0 & 0.1 & 0.2 & 1.0 \\
\cline { 2 - 7 } & -200 & 0.2 & 0.2 & 0.3 & 0.8 & $1.7: 0.20$ \\
\hline
\end{tabular}

Table 6.3.1: End-Effector Error $-R_{M}=25$ 


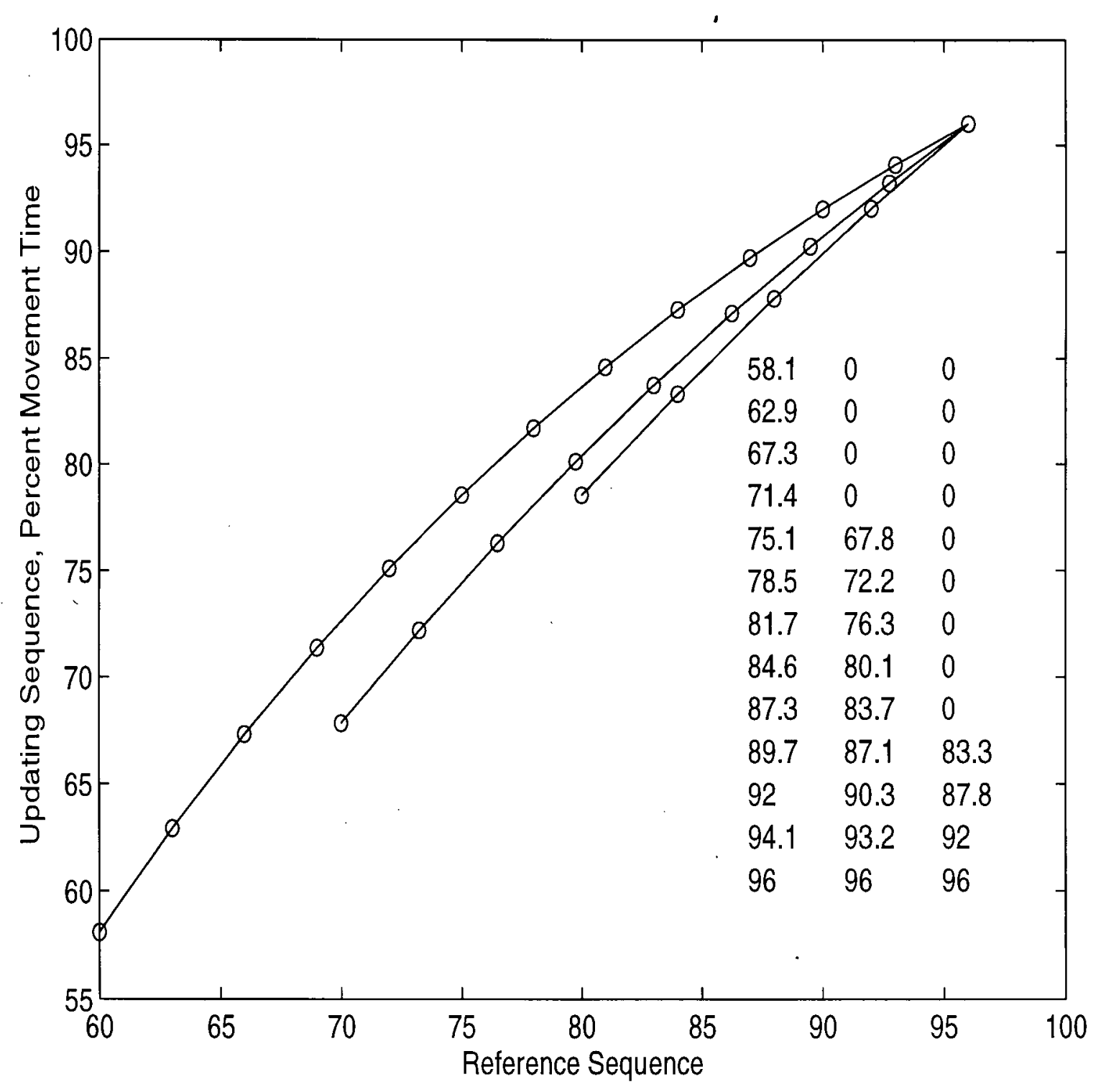

Figure 6.5: Updating Sequence vs. Reference Sequence 
For this mass ratio there were 25 different $(x, y)$ positions that the end-effector was commanded to servo to. The algorithm was set up to use multiple updating for the first movement and up to 5 endpoint servos to achieve a desired end-effector accuracy of $1.0 \mathrm{~cm}$ or less. Where there is only one value shown, this is the $A C C_{e}$ after the first 30 second movement. Where there are 2 values separated by a semi-colon (:), the first element is the error after the initial movement and the second value is the error after one 6 second endpoint servo. For a fairly large $R_{M}$ such as 25 , most of the movements achieve the desired accuracy after only one movement, and the others after one endpoint servo. The results from a series of simulations with $R_{M}$ equal to 3 are shown in Table 6.3.2.

\begin{tabular}{|c|l|l|l|l|l|l|}
\hline \multicolumn{7}{|c|}{ X desired position, cm } \\
\cline { 2 - 7 } & -200 & -100 & 0 & 100 & 200 \\
\cline { 2 - 7 } $\mathrm{s}$ & 200 & $99: 72: 67: 67$ & $80: 44: 41: 41$ & $61: 30: 17: 12: 11$ & $50: 25: 14: 8: 4$ & $42: 24: 15: 10: 6$ \\
\cline { 2 - 7 } $\mathrm{cm}$ & 100 & $51: 11: 2: 0.6$ & $27: 5: 1.6: 0.5$ & $24: 6: 1.9: 0.6$ & $14: 7: 4: 2: 1$ & $1.9: 0.8$ \\
\cline { 2 - 7 } & 0 & $17: 11: 2: 0.6$ & $6: 0.7$ & $7: 0.1$ & $2: 0.2$ & $8: 1.3: 0.3$ \\
\hline-100 & $4: 0.3$ & 1.0 & $1.3: 0.1$ & 0.8 & $9: 4: 1.9: 0.6$ \\
\hline & -200 & $11.8: 1.6: 0.4$ & $5: 0.5$ & $6: 0.8$ & $10: 2: 0.6$ & $18: 7: 3: 1.5: 0.7$ \\
\hline
\end{tabular}

Table 6.3.2: End-Effector Error $-R_{M}=3$

Clearly the smaller the $R_{M}$, the greater the number of iterations that must be performed to obtain the desired accuracy. Of the 25 desired points, only 1 point was achieved on the first iteration, and 19 others movements were successfully completed in less than five endpoint servos. For 2 points, the desired positions were achievable after a few more iterations, and 3 points cannot be achieved. This is because the desired point is no longer in the manipulator kinematic workspace (standard, 'fixed base type singularity), due to manipulator-base reorientation around the system centre of mass. There is code in the algorithm that detects this singuarity condition, 
and no more movements are performed. If joint range limits are incorporated $(+/-180$ degrees), and a joint angle exceeding the limit is required, then the movement is terminated as the desired point cannot be achieved. Simulations were performed with $R_{M}$ 's other than presented in the Tables 6.3.1 and 6.3.2, but are not shown as similar conclusions can be inferred from the previous discussion. In Figure 6.6 a system movement (desired point 200,-200) is shown in which 5 iterations are required to achieve an accuracy of less than $1 \mathrm{~cm}$. In Figure 6.7 a system . movement (desired point 0,200 ) is shown in which the desired point is no longer reachable after 4 iterations. The manipulator is in a singular configuration. To achieve the desired endeffector setpoint and required accuracy with a minimum number of endpoint servos, endpoint overcompensation (as discussed in Section 5.6) is utilized for movements with large $\Delta_{e}$ such as $(100,200)$ and $(200,200)$. These two points required more than five iterations to achieve the desired accuracy. If the end-effector error is greater than $25 \%$ of the total manipulator length after the first movement, $E_{O C}$ is used for the remaining iterations. For points that are no longer in the manipulator kinematic workspace, no difference is seen using $E_{o c}$. The results comparing the final error for movements with and without $E_{o c}$ are shown in Table 6.3.3.

\begin{tabular}{|r|r|r|}
\hline & $(100,200)$ & $(200,200)$ \\
\hline No $E_{o c}$ & $2.6 \mathrm{~cm}$ & $4.0 \mathrm{~cm}$ \\
\hline With $E_{o c}$ & $0.8 \mathrm{~cm}$ & $0.9 \mathrm{~cm}$ \\
\hline
\end{tabular}

Table 6.3.2: End-Effector Error - $E_{o c}$

Endpoint servoing in conjunction with endpoint overcompensation results in less iterations to achieve the desired accuracy. From the results presented in this chapter and from numerous other test simulations performed, it is evident that as long as the desired point is in the manipulators kinematic workspace and the joint range limits are not reached, a point can reached to any desired accuracy. The updating algorithm also enables the manipulator to achieve this 


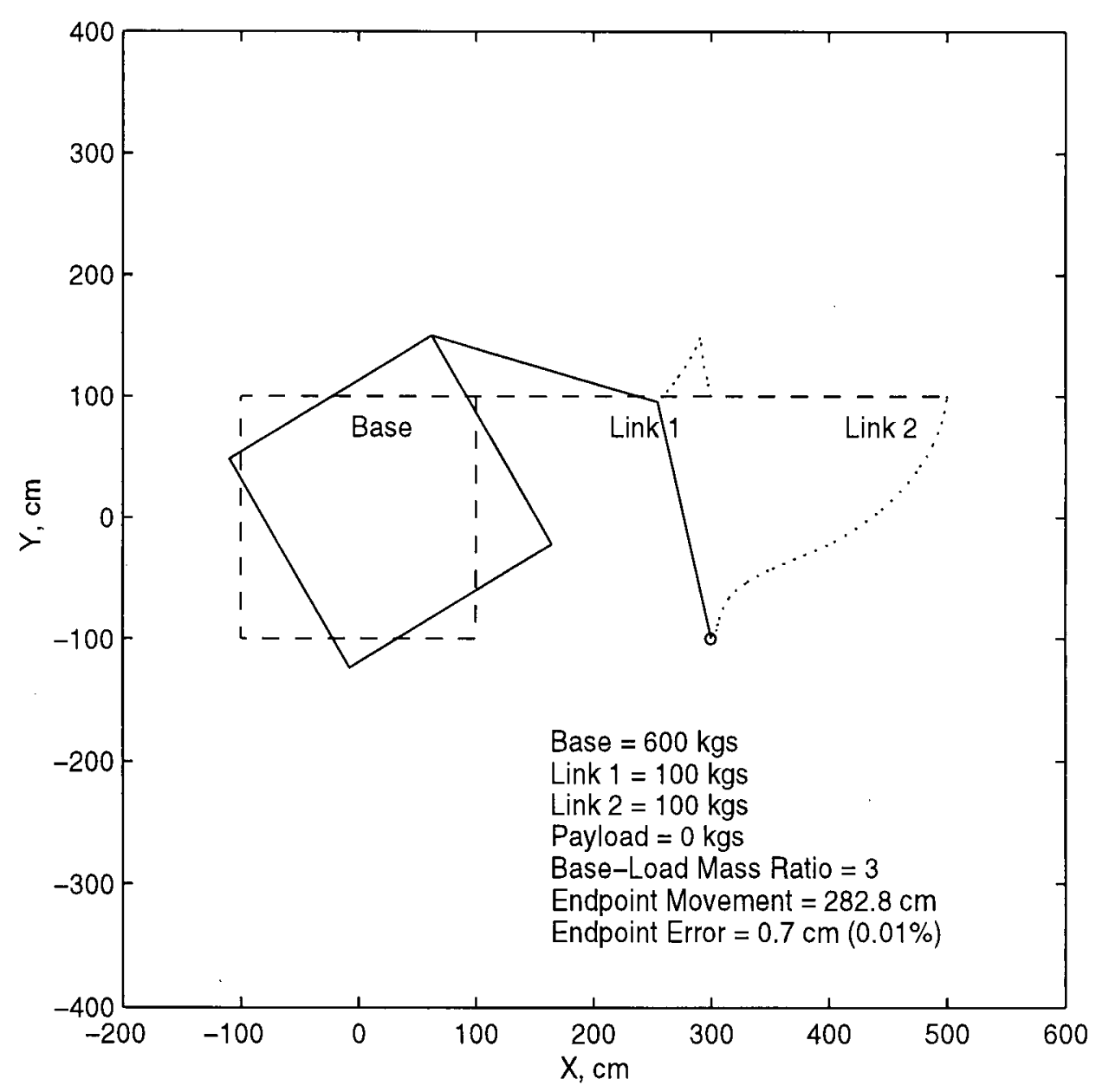

Figure 6.6: System Movement - Five Endpoint Servos 


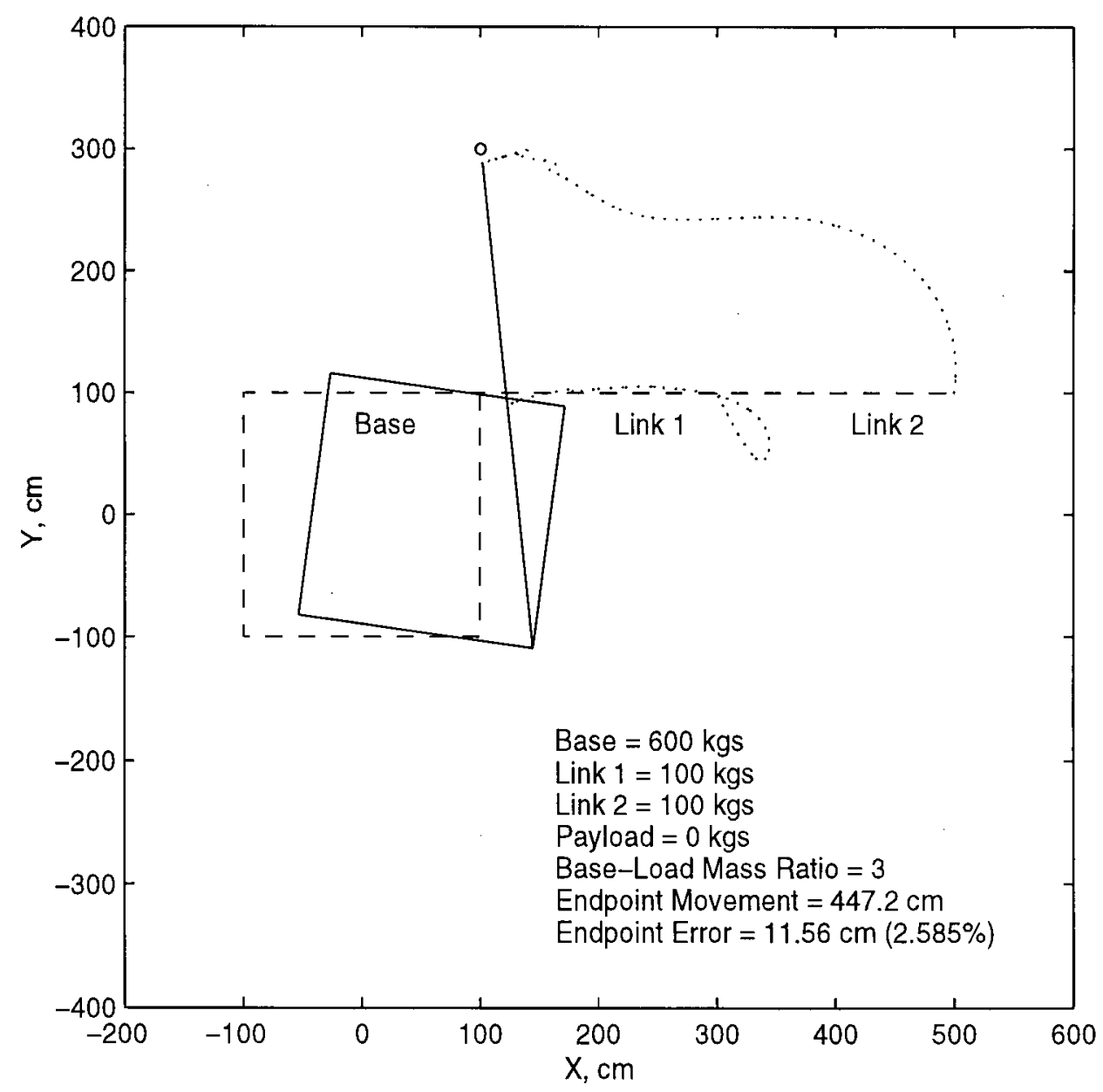

Figure 6.7: System Movement - Manipulator Singularity 
with a high degree of stability and a minimal amount of energy expended. 


\title{
Chapter 7
}

\author{
Conclusion
}

\subsection{Conclusions}

In this thesis, a motion control algorithm was developed for enabling a manipulator mounted on a non-fixed base to achieve a desired positon in inertial space. This is done without base control, and the exact dynamic parameters of the system are not needed. First, the generalized equations of motion have been derived for an $n$ link manipulator mounted on a non-fixed base in three dimensions. A joint trajectory generator utilizing velocity time scaling and quintic polynomials was developed. A simple PD position controller was used for controlling the manipulator and was shown to provide good servoing performance.

The motion control algorithm was developed based on experimental simulation results performed in Matlab. In the simulations, a two link revolute manipulator is attached to a planar (3 d.o.f.) base. The motion control technique incorporates the base motion without base motion control. Knowledge of the system dynamics is not required and the iterative inverse kinematics is performed online without model prediction.

Instead of performing a single inverse kinematic calculation at the beginning of a movement to determine the required joint setpoints, multiple inverse kinematic updates are done throughout a movement based on the motion control algorithm. The algorithm takes into account such factors as manipulator - base mass ratio, total required end-effector movement, and required 
accuracy and generates an updating sequence that starts at an optimal time. Updates are performed an specific times that result in better accuracy than multiple single inverse kinematic calculation movements.

Endpoint overcompensation and endpoint servoing were two techniques developed that enable any desired manipulator accuracy, assuming the manipulator does not encounter a kinematic or dynamic singularity. A large number of simulations were performed with varying manipulator-base mass ratios and desired end-effector points to verify the accuracy and stability of the algorithm.

\subsection{Recommendations for Future Work}

The following recommendations can be made: The motion control algorithm perfomance should be tested with a higher degree of freedom manipulator on a 3 dimensional base. For simulation purposes, the dynamic equations of motion for such a system were developed in this thesis and only need to be expanded.

The concept of energy recovery for space manipulator systems could be investigated. As briefly discussed in this thesis, electric generators could be used for braking the manipulator during the last part of a movement, rather than using the joint actuators for servoing. The energy would then be used for recharging a battery. The joint trajectories required to reach a setpoint using this method would have to be determined. If such a recovery system was not used, research could be done to see how joint friction could similarly be used for bringing the manipulator joints to a stop. In this thesis, joint actuator friction was not modelled.

The concept of dynamic singularities and manipulator workspace for non-fixed robotic systems could be incorporated into the motion control algorithm and trajectory generator. If a desired point cannot be reached by the manipulator at some point in a direct point to point 
movement, the manipulator may be able to reach the point using another path. These concepts are discussed in detail in [Papa 93] and [Naka 89].

An experimental testbed could be set up to verify the techniques presented in this thesis and to experiment with other methods. Alternatively, the motion control algorithm performance could be tested on an existing testbed. Several universities already have setups for testing planar non-fixed base robotic manipulators. 


\section{Bibliography}

[Alex 87] Alexander, H.L.,Cannon, R.H. Jr. 1987. Experiments on the control of a satellite manipulator. Proc. 1987 American Control Conf.

[An 88] An, Chae H., Atkeson, Christopher G., Hollerbach, John M., 1988. Model Based Control of a Robot Manipulator. Cambridge, Massachusetts: MIT Press.

[Bron 86] Bronez, M.A., Clarke, M.M., and Quinn, A. 1986. Requirements development for a free-flying robot-the 'ROBIN'. Proc. IEEE Int. Conf. on Robotics and Automation.

[Crai 86] Craig, John J., 1986. Introduction to Robotics. Reading, Massachusetts: AddisonWesley Publishing Company.

[Daws 90] Dawson, D.M., March 1990. Uncertainties in the control of robot manipulators. Ph.D. Thesis, School of Electrical Engineering, Georgia Institute of Technology.

[Dubo 91] Dubowsky, Steven, and Torres, Miguel A. 1991. Path Planning For Space Manipulators To Minimize Spacecraft Attitude Disturbances. IEEE Int. Conf. on Robotics and Automation 2522-2528.

[Egel 87] Egeland, O. 1987. Task-space tracking with redundant manipulators. IEEE J. Robot. Automation RA-3(5):471-475.

[Egel 93] Egeland, O., and Sagli, J.R. 1993. Coordination of motion in a spacecraftmanipulator system. Int. J. Robot. Res. 12(4):366-379.

[Fern 92] Fernandes, C., and Gurvits, L., and Li, Z.X. 1992. Attitude Control of Space Platform/Manipulator System Using Internal Motion. IEEE Int. Conf. on Robotics and Automation 893-898.

[Fren 85] French, R, and Boyce, B. 1985. Satellite servicing by teleoperators. J. Engin. Industry 107:49-54.

[Fu 87] Fu, K.S., Gonzalez, R.C., and Lee, C.S.G. 1987. Robotics: Control, Sensing, Vision, and Intelligence. New York: McGraw Hill.

[Holl 84] Hollerbach, John M. 1984. Dynamic Scaling of Manipulator Trajectories. ASME J. Dyn. Systems, Measurement and Control, vol. 106, pp. 102-106.

[Hugh 86] Hughes, P., 1986. Spacecraft Attitude Dyanmics. Wiley, New York, NY. 
[Khat 88] Khatib, O. 1988. augmented object and reduced effective inertia in robot systems. Proc. American Control Conference, pp. 2140-2147.

[Lee 85] Lee, S., Bekey, G., and Bejczy, A.K. 1985. Computer Control of space-borne teleoperators with sensory feedback. Proc. IEEE Int. Conf. on Robotics and Automation.

[Lewi 93] Lewis, F.L., Abdallah, C.T., and Dawson, D.M. 1993. Control of Robot Manipulators. New York: Macmillan Publishing Company.

[Long 87] Longman, R.W., Lindberg, R.E., and Zedd, M.F. 1987. Satellite-mounted robot manipulators-New kinematics and reaction moment compensation. Int. J. Robot. Res. 6(3):87-103.

[Nags 92] Nagashima, Fumio Z., and Nakamura, Yoshiteru 1992. Efficient Computation Scheme for the Kinematics and Inverse Dynamics of a Satellite-Based Manipulator. IEEE Int. Conf. on Robotics and Automation 905-912.

[Naka 89] Nakamura, Yoshihiko, Mukherjee, Ranjan 1989. Nonholonomic Path Planning of Space Robots. IEEE Int. Conf. on Robotics and Automation 1050-1055.

[Paul 81] Paul, R.P. 1981. Robot Manipulator: Mathematics, Programming and Control. MIT Press, Cambridge, Massachusetts.

[Papa 93] Papadopoulos, E., Dubowsky, S. 1993. Dynamic Singularities in Free-Floating Space Manipulators. ASME J. Dyn. Systems, Measurement and Control, vol. 115, pp. 4452.

[Papa 90] Papadopoulos, E., 1990. On the Dynamics and Control of Space Manipulators. Ph.D. Thesis, Department of Mechanical Engineering, MIT

[Sali 85] Salisbury, J.K., and Abramowitz, J.D. 1985. Design and control of a redundant mechanism for small motion. Proc. IEEE Int. Conf. on Robotics and Automation pp. 323-328.

[Spon 89] Spong, M.W., and Vidyasagar, M. 1989. Robot Dynamics and Control. New York: John Wiley.

[Umet 87] Umetani, Y., and Yoshida, K. 1989. Experimental study on two dimensional freeflying robot satellite model. NASA Space Telerobotics Workshop, Jet Propulsion Laboratory.

[Vafa 90] Vafa, Z., and Dubowsky, S. 1990. The Kinematics and Dynamics of Space Manipulators: The Virtual Manipulator Approach. Int. J. Robot. Res. 9(4):3-21.

[Xu 93] Xu, Yangsheng 1993. The Measure Of Dynamic Coupling Of Space Robots. IEEE Int. Conf. on Robotics and Automation 615-620. 


\section{Appendix A}

\section{Full Equations of Motion : 2-D}

The three base dynamic equations of motion, expanded from Equation 3.36, are presented below with $z_{1}$ and $z_{2}$ being the base translational coordinates, $z_{3}$ denoting the base rotaional coordinate, and $q_{1}$ and $q_{2}$ denoting the revolute link coordinates.

$$
\begin{aligned}
& Z 1: 0=(m b+m 1+m p+m 2) \ddot{z}_{1}+((-m p-m 2-m 1) \sin (z 3) R b 1 \\
& +(-m p-m 2-m 1) \cos (z 3) R b 2+\left(-m p l 1-\frac{1}{2} m 1 l 1-m 2 l 1\right) \sin (z 3+q 1) \\
& \left.+\left(-\frac{1}{2} m 212-m p l 2\right) \sin (z 3+q 1+q 2)\right) \ddot{z}_{3}+( \\
& \left(-m p l 1-\frac{1}{2} m 1 l 1-m 2 l 1\right) \sin (z 3+q 1) \\
& \left.+\left(-\frac{1}{2} m \mathscr{2} l \mathscr{2}-m p l \mathbb{2}\right) \sin (z 3+q 1+q \mathscr{2})\right) \ddot{q}_{1} \\
& +\left(-\frac{1}{2} m 2 l 2-m p l 2\right) \sin (z 3+q 1+q 2) \ddot{q}_{2} \\
& +\left(-\dot{z}_{3}^{2} m 2-\dot{z}_{3}^{2} m p-\dot{z}_{3}^{2} m 1\right) \cos (z 3) R b 1 \\
& +\left(\dot{z}_{3}^{2} m 1+\dot{z}_{3}^{2} m \mathscr{2}+\dot{z}_{3}^{2} m p\right) \sin (z 3) R b \mathscr{2}+( \\
& \left(-\frac{1}{2} \dot{z}_{3}^{2}-\dot{q}_{1} \dot{z}_{3}-\frac{1}{2} \dot{q}_{1}^{2}\right) l 1 m 1+\left(-\dot{z}_{3}^{2}-\dot{q}_{1}^{2}-2 \dot{q}_{1} \dot{z}_{3}\right) l 1 m 2 \\
& \left.+\left(-2 \dot{q}_{1} \dot{z}_{3} m p-\dot{z}_{3}^{2} m p-\dot{q}_{1}^{2} m p\right) l 1\right) \cos (z 3+q 1)+( \\
& \left(-\frac{1}{2} \dot{q}_{1}^{2}+\left(-\dot{q}_{2}-\dot{z}_{3}\right) \dot{q}_{1}-\frac{1}{2} \dot{z}_{3}^{2}-\dot{q}_{2} \dot{z}_{3}-\frac{1}{2} \dot{q}_{\mathscr{Q}}^{2}\right) \text { l2 } m \mathscr{2}+( \\
& -\dot{q}_{1}^{2} m p+\left(-2 \dot{q}_{2} m p-2 \dot{z}_{3} m p\right) \dot{q}_{1}-\dot{z}_{3}^{2} m p-\dot{q}_{2}^{2} m p
\end{aligned}
$$


$\left.\left.-2 \dot{q}_{2} \dot{z}_{3} m p\right) l 2\right) \cos (z 3+q 1+q 2)$

$$
\begin{aligned}
Z 2: & =(m 1+m 2+m b+m p) \ddot{z}_{2}+((m p+m 1+m 2) \cos (z 3) R b 1 \\
& +(-m 2-m 1-m p) \sin (z 3) R b 2+\left(\frac{1}{2} m 1 l 1+m 2 l 1+m p l 1\right) \cos (z 3+q 1) \\
& \left.+\left(m p l 2+\frac{1}{2} m 2 l 2\right) \cos (z 3+q 1+q 2)\right) \ddot{z}_{3}+( \\
& \left(\frac{1}{2} m 1 l 1+m 2 l 1+m p l 1\right) \cos (z 3+q 1) \\
& \left.+\left(m p l 2+\frac{1}{2} m 2 l 2\right) \cos (z 3+q 1+q 2)\right) \ddot{q}_{1} \\
& +\left(m p l 2+\frac{1}{2} m 2 l 2\right) \cos (z 3+q 1+q 2) \ddot{q}_{2} \\
& +\left(-\dot{z}_{3}^{2} m 2-\dot{z}_{3}^{2} m 1-\dot{z}_{3}^{2} m p\right) \sin (z 3) R b 1 \\
& +\left(-\dot{z}_{3}^{2} m 2-\dot{z}_{3}^{2} m 1-\dot{z}_{3}^{2} m p\right) \cos (z 3) R b 2+( \\
& \left(-\frac{1}{2} \dot{q}_{1}^{2}-\frac{1}{2} \dot{z}_{3}^{2}-\dot{q}_{1} \dot{z}_{3}\right) l 1 m 1+\left(-\dot{q}_{1}^{2}-2 \dot{q}_{1} \dot{z}_{3}-\dot{z}_{3}^{2}\right) l 1 m 2 \\
& \left.+\left(-\dot{z}_{3}^{2} m p-2 \dot{q}_{1} \dot{z}_{3} m p-\dot{q}_{1}^{2} m p\right) l 1\right) \sin (z 3+q 1)+( \\
& \left(-\frac{1}{2} \dot{q}_{1}^{2}+\left(-\dot{q}_{2}-\dot{z}_{3}\right) \dot{q}_{1}-\frac{1}{2} \dot{q}_{2}^{2}-\dot{q}_{2} \dot{z}_{3}-\frac{1}{2} \dot{z}_{3}^{2}\right) l 2 m \mathscr{2}+( \\
& -\dot{q}_{1}^{2} m p+\left(-2 \dot{q}_{2} m p-2 \dot{z}_{3} m p\right) \dot{q}_{1}-\dot{q}_{2}^{2} m p-\dot{z}_{3}^{2} m p \\
& \left.\left.-2 \dot{q}_{2} \dot{z}_{3} m p\right) l 2\right) \sin (z 3+q 1+q 2)
\end{aligned}
$$

$$
\begin{aligned}
Z 3: 0 & =((-m 2-m 1-m p) \sin (z 3) R b 1+(-m 2-m 1-m p) \cos (z 3) R b \mathscr{2} \\
& +\left(-m p l 1-m 2 l 1-\frac{1}{2} m 1 l 1\right) \sin (z 3+q 1) \\
& \left.+\left(-\frac{1}{2} m 2 l 2-m p l 2\right) \sin (z 3+q 1+q 2)\right) \ddot{z}_{1}+( \\
& (m p+m 1+m 2) \cos (z 3) R b 1+(-m 2-m 1-m p) \sin (z 3) R b \mathscr{2} \\
& +\left(\frac{1}{2} m 1 l 1+m 2 l 1+m p l 1\right) \cos (z 3+q 1)
\end{aligned}
$$




$$
\begin{aligned}
& \left.+\left(m p l 2+\frac{1}{2} m 2 l 2\right) \cos (z 3+q 1+q 2)\right) \ddot{z}_{2}+\left((m p+m 1+m 2) R b 1^{2}\right. \\
& +\left(\cos (q 1) m 1 l 1+\left(2 l 1 \cos (q 1)+l 2 \cos \left(q 1+q^{2}\right)\right) m^{2}\right. \\
& +2 m p l \mathscr{2} \cos (q 1+q \mathscr{2})+2 m p l 1 \cos (q 1)) R b 1+(m p+m 1+m \mathscr{2}) R b \mathscr{2}^{2} \\
& +(\sin (q 1) m 1 l 1+(l 2 \sin (q 1+q 2)+2 l 1 \sin (q 1)) m 2 \\
& +2 m p l 2 \sin (q 1+q 2)+2 m p l 1 \sin (q 1)) R b \mathscr{2}+\frac{1}{3} m 1 l 1^{2} \\
& +\left(l \mathscr{2} l 1 \cos (q \mathscr{2})+\frac{1}{3} l \mathscr{Q}^{2}+l 1^{2}\right) m \mathscr{2}+\frac{1}{3} m b r b \mathscr{2}^{2}+\frac{1}{3} m b r b 1^{2}+l 1^{2} m p \\
& \left.+l 2^{2} m p+2 l 2 m p l 1 \cos (q 2)\right) \ddot{z}_{3}+\left(\left(\frac{1}{2} \cos (q 1) m 1 l 1\right.\right. \\
& +\left(l 1 \cos (q 1)+\frac{1}{2} l 2 \cos (q 1+q 2)\right) m 2+m p l 1 \cos (q 1) \\
& +m p l 2 \cos (q 1+q 2)) R b 1+\left(\frac{1}{2} \sin (q 1) m 1 l 1\right. \\
& +\left(l 1 \sin (q 1)+\frac{1}{2} l 2 \sin (q 1+q 2)\right) m 2+m p l 1 \sin (q 1) \\
& +m p l 2 \sin (q 1+q 2)) R b 2+\frac{1}{3} m 1 l 1^{2}+\left(l 2 l 1 \cos (q 2)+\frac{1}{3} l 2^{2}+l 1^{2}\right) m 2 \\
& \left.+l 1^{2} m p+2 l 2 m p l 1 \cos \left(q^{2}\right)+l 2^{2} m p\right) \ddot{q}_{1}+( \\
& \left(m p l \mathscr{2} \cos (q 1+q \mathscr{2})+\frac{1}{2} \cos (q 1+q \mathscr{2}) m \mathscr{2} l \mathscr{2}\right) R b 1 \\
& +\left(\frac{1}{2} \sin (q 1+q 2) m 2 l 2+m p l 2 \sin (q 1+q 2)\right) R b 2 \\
& \left.+\left(\frac{1}{2} l 2 l 1 \cos (q \mathscr{2})+\frac{1}{3} l \mathscr{2}^{2}\right) m 2+l 2 m p l 1 \cos (q \mathscr{2})+l 2^{2} m p\right) \ddot{q}_{2}+( \\
& \left(-\dot{q}_{1} \dot{z}_{3} \sin (q 1)-\frac{1}{2} \dot{q}_{1}^{2} \sin (q 1)\right) l 1 m 1+( \\
& \left(-2 \dot{q}_{1} \dot{z}_{3} \sin (q 1)-\dot{q}_{1}^{2} \sin (q 1)\right) l 1+\left(-\frac{1}{2} \dot{q}_{1}^{2} \sin \left(q 1+q^{2}\right)\right. \\
& +\left(-\dot{q}_{2} \sin \left(q 1+q^{2}\right)-\dot{z}_{3} \sin (q 1+q 2)\right) \dot{q}_{1}-\frac{1}{2} \dot{q}_{\mathscr{2}}^{2} \sin \left(q 1+q^{2}\right) \\
& \left.\left.-\dot{q}_{2} \dot{z}_{3} \sin (q 1+q 2)\right) l 2\right) m 2 \\
& +\left(-\dot{q}_{1}^{2} m p \sin (q 1)-2 \dot{q}_{1} \dot{z}_{3} m p \sin (q 1)\right) l 1+\left(-\dot{q}_{1}^{2} \sin (q 1+q 2) m p\right. \\
& +\left(-2 \dot{q}_{2} \sin \left(q 1+q^{2}\right) m p-2 \dot{z}_{3} m p \sin \left(q 1+q^{2}\right)\right) \dot{q}_{1} \\
& \left.\left.-\dot{q}_{2}^{2} \sin \left(q 1+q^{2}\right) m p-2 \dot{q}_{2} \dot{z}_{3} m p \sin \left(q 1+q^{2}\right)\right) l 2\right) R b 1+(
\end{aligned}
$$




$$
\begin{aligned}
& \left(\frac{1}{2} \dot{q}_{1}^{2} \cos (q 1)+\dot{q}_{1} \dot{z}_{3} \cos (q 1)\right) l 1 m 1+( \\
& \left(\dot{q}_{1}^{2} \cos (q 1)+2 \dot{q}_{1} \dot{z}_{3} \cos (q 1)\right) l 1+\left(\frac{1}{2} \dot{q}_{1}^{2} \cos (q 1+q 2)\right. \\
& +\left(\dot{q}_{2} \cos (q 1+q 2)+\dot{z}_{3} \cos (q 1+q 2)\right) \dot{q}_{1}+\dot{q}_{2} \dot{z}_{3} \cos (q 1+q 2) \\
& \left.\left.+\frac{1}{2} \dot{q}_{\mathscr{L}}^{2} \cos (q 1+q \mathscr{2})\right) l \mathscr{2}\right) m \mathscr{2} \\
& +\left(\dot{q}_{1}^{2} m p \cos (q 1)+2 \dot{q}_{1} \dot{z}_{3} m p \cos (q 1)\right) l 1+\left(\dot{q}_{1}^{2} \cos (q 1+q \mathscr{2}) m p\right. \\
& +\left(2 \dot{z}_{3} m p \cos \left(q 1+q^{2}\right)+2 \dot{q}_{2} \cos \left(q 1+q^{2}\right) m p\right) \dot{q}_{1} \\
& \left.\left.+2 \dot{q}_{2} \dot{z}_{3} m p \cos (q 1+q 2)+\dot{q}_{2}^{2} \cos (q 1+q 2) m p\right) l 2\right) R b 2 \\
& +\left(-\frac{1}{2} \dot{q}_{2}^{2} \sin \left(q_{2}\right)-\dot{q}_{2} \dot{z}_{3} \sin \left(q^{2}\right)-\dot{q}_{1} \dot{q}_{2} \sin \left(q^{2}\right)\right) l 2 l 1 m \mathscr{2}+ \\
& \left(-2 \dot{q}_{2} \dot{z}_{3} m p \sin \left(q^{2}\right)-2 \dot{q}_{1} \dot{q}_{2} m p \sin \left(q^{2}\right)-\dot{q}_{2}^{2} m p \sin \left(q^{2}\right)\right) l 2 l 1
\end{aligned}
$$

Similarily, the two link dynamic equations of motion, expanded from Equation 3.34, are presented below

$$
\begin{aligned}
& Q 1: \tau_{1}=\left(\left(-m p l 1-m 2 l 1-\frac{1}{2} m 1 l 1\right) \sin (z 3+q 1)\right. \\
& \left.+\left(-\frac{1}{2} m 2 l 2-m p l 2\right) \sin (z 3+q 1+q 2)\right) \ddot{z}_{1}+( \\
& \left(\frac{1}{2} m 1 l 1+m 2 l 1+m p l 1\right) \cos (z 3+q 1) \\
& \left.+\left(m p l 2+\frac{1}{2} m 2 l 2\right) \cos (z 3+q 1+q 2)\right) \ddot{z}_{2}+\left(\left(\frac{1}{2} \cos (q 1) m 1 l 1\right.\right. \\
& +\left(l 1 \cos (q 1)+\frac{1}{2} l 2 \cos (q 1+q 2)\right) m 2+m p l 1 \cos (q 1) \\
& +m p l 2 \cos (q 1+q 2)) R b 1+\left(\frac{1}{2} \sin (q 1) m 1 l 1\right. \\
& +\left(l 1 \sin (q 1)+\frac{1}{2} l 2 \sin (q 1+q 2)\right) m 2+m p l 1 \sin (q 1) \\
& +m p l 2 \sin (q 1+q 2)) R b \mathscr{2}+\frac{1}{3} m 1 l 1^{2}+\left(l 2 l 1 \cos (q 2)+\frac{1}{3} l \mathscr{2}^{2}+l 1^{2}\right) m \mathscr{2} \\
& \left.+l 1^{2} m p+2 l 2 m p l 1 \cos \left(q^{2}\right)+l 2^{2} m p\right) \ddot{z}_{3}+\left(\frac{1}{3} m 1 l 1^{2}\right.
\end{aligned}
$$




$$
\begin{aligned}
& +\left(l 2 l 1 \cos (q 2)+\frac{1}{3} l 2^{2}+l 1^{2}\right) m 2+2 l 2 m p l 1 \cos (q 2)+l 2^{2} m p+l 1^{2} m p \\
& ) \ddot{q}_{1}+\left(\left(\frac{1}{2} l 2 l 1 \cos (q 2)+\frac{1}{3} l 2^{2}\right) m 2+l 2 m p l 1 \cos (q \mathscr{2})+l 2^{2} m p\right) \ddot{q}_{2} \\
& +\left(\frac{1}{2} \dot{z}_{3}^{2} \sin (q 1) m 1 l 1+\left(\frac{1}{2} \dot{z}_{3}^{2} \sin (q 1+q 2) l 2+\dot{z}_{3}^{2} \sin (q 1) l 1\right) m 2\right. \\
& \left.+\dot{z}_{3}^{2} m p l 2 \sin (q 1+q 2)+\dot{z}_{3}^{2} m p l 1 \sin (q 1)\right) R b 1+( \\
& -\frac{1}{2} \dot{z}_{3}^{2} \cos (q 1) m 1 l 1+\left(-\dot{z}_{3}^{2} \cos (q 1) l 1-\frac{1}{2} \dot{z}_{3}^{2} \cos (q 1+q 2) l 2\right) m 2 \\
& \left.-\dot{z}_{3}^{2} m p l 1 \cos (q 1)-\dot{z}_{3}^{2} m p l 2 \cos (q 1+q 2)\right) R b 2 \\
& +\left(-\frac{1}{2} \dot{q}_{2}^{2} \sin (q 2)-\dot{q}_{2} \dot{z}_{3} \sin (q 2)-\dot{q}_{1} \dot{q}_{2} \sin (q 2)\right) l \mathscr{2} l 1 m \mathscr{2}+ \\
& \left(-2 \dot{q}_{2} \dot{z}_{3} m p \sin (q 2)-2 \dot{q}_{1} \dot{q}_{2} m p \sin \left(q^{2}\right)-\dot{q}_{2}^{2} m p \sin (q 2)\right) l 2 l 1
\end{aligned}
$$

$$
\begin{aligned}
& \text { Q2: } \tau_{2}=\left(-\frac{1}{2} m 2 l 2-m p l 2\right) \sin (z 3+q 1+q 2) \ddot{z}_{1} \\
& +\left(m p l 2+\frac{1}{2} m 2 l 2\right) \cos (z 3+q 1+q 2) \ddot{z}_{2}+( \\
& \left(m p l 2 \cos (q 1+q 2)+\frac{1}{2} \cos (q 1+q 2) m 2 l 2\right) R b 1 \\
& +\left(\frac{1}{2} \sin (q 1+q 2) m 2 l 2+m p l 2 \sin (q 1+q 2)\right) R b 2 \\
& \left.+\left(\frac{1}{2} l 2 l 1 \cos (q 2)+\frac{1}{3} l 2^{2}\right) m 2+l 2 m p l 1 \cos (q 2)+l 2^{2} m p\right) \ddot{z}_{3} \\
& +\left(\left(\frac{1}{2} l 2 l 1 \cos (q 2)+\frac{1}{3} l 2^{2}\right) m 2+l 2 m p l 1 \cos (q 2)+l 2^{2} m p\right) \ddot{q}_{1} \\
& +\left(l \mathscr{2}^{2} m p+\frac{1}{3} m 2 l^{2}\right) \ddot{q}_{2} \\
& +\left(\frac{1}{2} \dot{z}_{3}^{2} \sin (q 1+q 2) m 2 l 2+\dot{z}_{3}^{2} m p l 2 \sin (q 1+q 2)\right) R b 1 \\
& +\left(-\frac{1}{2} \dot{z}_{3}^{2} \cos \left(q 1+q^{2}\right) m 2 l 2-\dot{z}_{3}^{2} m p l 2 \cos (q 1+q 2)\right) R b 2 \\
& +\left(\dot{q}_{1} \dot{z}_{3} \sin (q 2)+\frac{1}{2} \dot{z}_{3}^{2} \sin \left(q^{2}\right)+\frac{1}{2} \dot{q}_{1}^{2} \sin \left(q^{2}\right)\right) l \mathscr{2} l 1 m^{2} \\
& +\left(\dot{q}_{1}^{2} m p \sin \left(q^{2}\right)+2 \dot{q}_{1} \dot{z}_{3} m p \sin (q \mathscr{L})+\dot{z}_{3}^{2} m p \sin \left(q^{\mathscr{L}}\right)\right) l \mathbb{2} l 1
\end{aligned}
$$


Appendix B

Matlab Files 


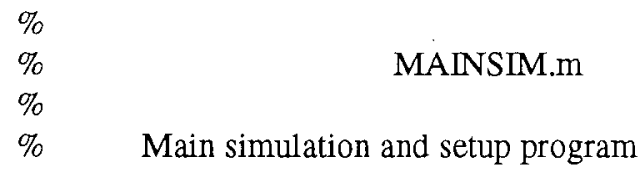

clear;clear global;clc;cf;

echo off,more off

global $\mathrm{m} 1 \mathrm{~m} 2 \mathrm{mp} 1112 \mathrm{Rb} 1 \mathrm{Rb} 2 \mathrm{rb} 1 \mathrm{rb} 2$ tfin ggg mb endeffx endeffy ... endeffxx endeffyy thj 1 thj 2 vf $1 r$ vf $2 r$ af $1 \mathrm{r}$ af $2 r$...

tinit $\mathrm{AB}$ maxthdp1 maxthdp2 minthdp1 minthdp2 tstep finish mp tq1max tq2max ... timlen stpcnter timematrix outworkspace docgcheck doelbowcheck tfin ... ac tq err tqlast allowederror doservo doovercomp velscale dotorquescale ... servocount

\% Loops are set up to vary the base-manipulator mass ratio

$\%$ and the end-effector desired pint in inertial space

for MRcnter=1:3,

for $x c n t e r=1: 5$,

for $y c n t e r=1: 5$,

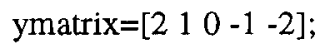

yyy=ymatrix (ycnter)

yynam $=[$ int2str(yyy)];

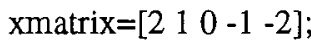

$\mathrm{xxx}=\mathrm{xmatrix}(\mathrm{xcnter})$

$\mathrm{xxnam}=[\operatorname{int} 2 \mathrm{str}(\mathrm{xxx})]$

MRmatrix $=\left[\begin{array}{lll}3 & 7 & 15\end{array}\right]$

MR=MRmatrix(MRcnter)

$\mathrm{mbb}=[\mathrm{int} 2 \mathrm{str}(\mathrm{MR})]$;

tstart $=0$;

tfin=30; $\quad \%$ Movement total time

doservo $=1 ; \quad \%$ Endpoint servoing flag

dotorquescale $=1 ; \%$ Torque actutor limits flag

allowederror $=1 ; \%$ Desired end-effector accuracy in $(\mathrm{cm})$.

$\%$ Endpoint servoing will repeat until this value is reached

doovercomp $=0 ; \%$ Endpoint overcompensation flag

doelbowcheck $=0 ; \%$ Variable $=1$ if arm in elbow 'up' configuration

servocount $=0$;

initvars; \% Call $\mathrm{m}$-file to initialize and define all masses, link lengths, initial conditions

$m p=0$

$\%$ Payload mass

$\mathrm{mb}=(\mathrm{m} 1+\mathrm{m} 2+\mathrm{mp}) * \mathrm{MR} ; \quad \%$ Base mass calculated from base-manip mass ratio

$\%$ and link masses in initvars.m 
$\%$ Initial condition vector

$y=[\mathrm{r} 10 \mathrm{r} 10 \mathrm{dot}$ r $20 \mathrm{r} 20 \mathrm{dot}$ phr phrdot thr10 thr10dot thr20 thr20dot];

desendef; $\quad \%$ M-file to determine desired the end-effector position in inertial (fixed) frame

$\mathrm{t}=0$

upd; $\%$ Call algorithm to determine the updating sequence

timlen=length(timematrix); $\quad \%$ Number of elements of time step matrix

tfin=timematrix (timlen); $\quad \%$ tfin - last element of timematrix

$\%$ Begin the first movement. It is broken in segments, depending on the updating sequence

$\%$ If 10 segments, this will loop 10 times.

for stpcnter=1:timlen-1,

tinit1=timematrix(stpcnter);

recalc4; $\quad \% \mathrm{M}$-file to determine inertial desired point in manip frame

$\%$ and to perform inverse kinematics

updatedegrees(stpcnter,:)=[thj1*180/pi thj2*180/pi]; \% Data vector for troubleshooting

$\%$ M-file called to determine quintic polynomial coefficients, based on initial and final $\%$ angular conditions, and start and finish time of this segment

$[A B]=$ trajpln (tfin, tinit 1,thr 10, thr 10dot, acc 10, thr20,thr20dot, ... acc20,thj1,vf1r,af1r,thj2,vf2r,af2r);

$\mathrm{y} 0=[\mathrm{r} 10 \mathrm{r} 10 \mathrm{dot} \mathrm{r} 20 \mathrm{r} 20 \mathrm{dot}$ phr phrdot thr 10 thr $10 \mathrm{dot}$ thr20 thr20dot];

if stpcnter $>=2, \quad \%$ Here the segments are pieced together, since each

$\mathrm{z}=$ length $(\mathrm{y}(:, 10))$;

$\%$ segment integrated seperately by ode 23 mik.m

tmov=t;ymov=y;accmov=aout;

tqmov=tqout; errmov=errout;

end

$\%$ Runge Kutta integrator called to integrate dynamic equations of motion, found in file $\%$ dynceqn2.m, for current segment

[t,y,aout,tqout,errout $]=$ ode23mik('dynceqn2', timematrix (stpcnter),...

$\mathrm{z} 1=$ length $(\mathrm{y}(:, 10))$; timematrix(stpcnter+1),y0);

if stpcnter $>=2, \quad \%$ Here the segments are pieced together $\mathrm{t}=[\mathrm{tmov} ; \mathrm{t}(2: \mathrm{z} 1)]$;

$\mathrm{y}=[\mathrm{ymov} ; \mathrm{y}(2: \mathrm{zl},:)]$;

aout=[accmov;aout $(2: z 1,:)]$; 


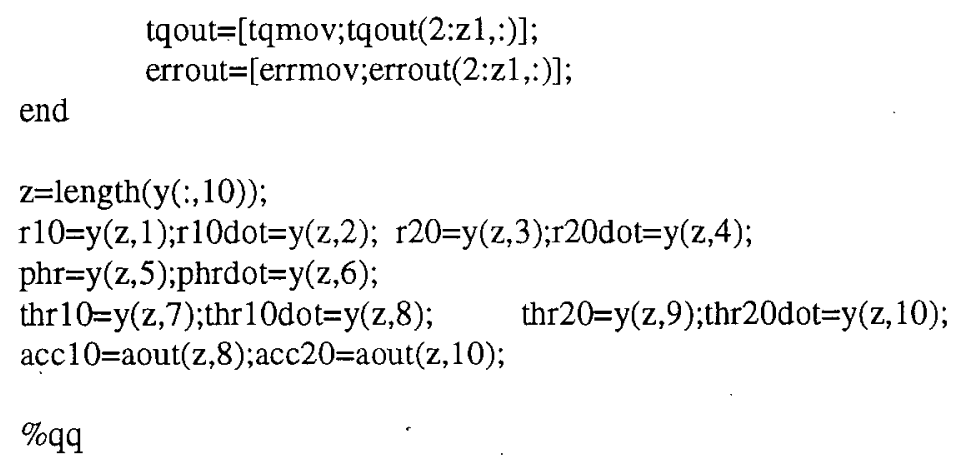

$\%$ First main movement now completed

calctrj2; \% Base and link tip trajectories are calculated

errors; \% Endpoint distance and errors calculated

$\%$ Data vectors

timematrixmat(:,1)=[timematrix'];

lerrormat $(:, 1)=$ lerror;

matnum $=1$;

oldtimlen=timlen;

$\%$ Endpoint servoing performed of flag set

if doservo $==1$,

servotime $=0.2 *$ tfin;

$\%$ Endpoint servoing loops until desired accuracy achieved

$\%$ or for a set number of loops - servocount is loop counter

while lerror $>$ allowederror, $\%$ in $\mathrm{cm}$

servocount=servocount $+1 ; \%=1$ is the start of first servo

tstart=t(z); \% Time at end of last movement

tfin=tstart+servotime;

upd; \% Call algorithm to determine the updating sequence

$\%$ The seuqence deterimed here will set the number of updates in the

$\%$ servoing movement

timlen=length(timematrix);

$\%$ If 10 updates in the E>P. servo movement, this will loop 10 times.

$\%$ Inner workings of the loop the same as the previous loop for

$\%$ the first main movement

for stpcnter=1:timlen-1, 


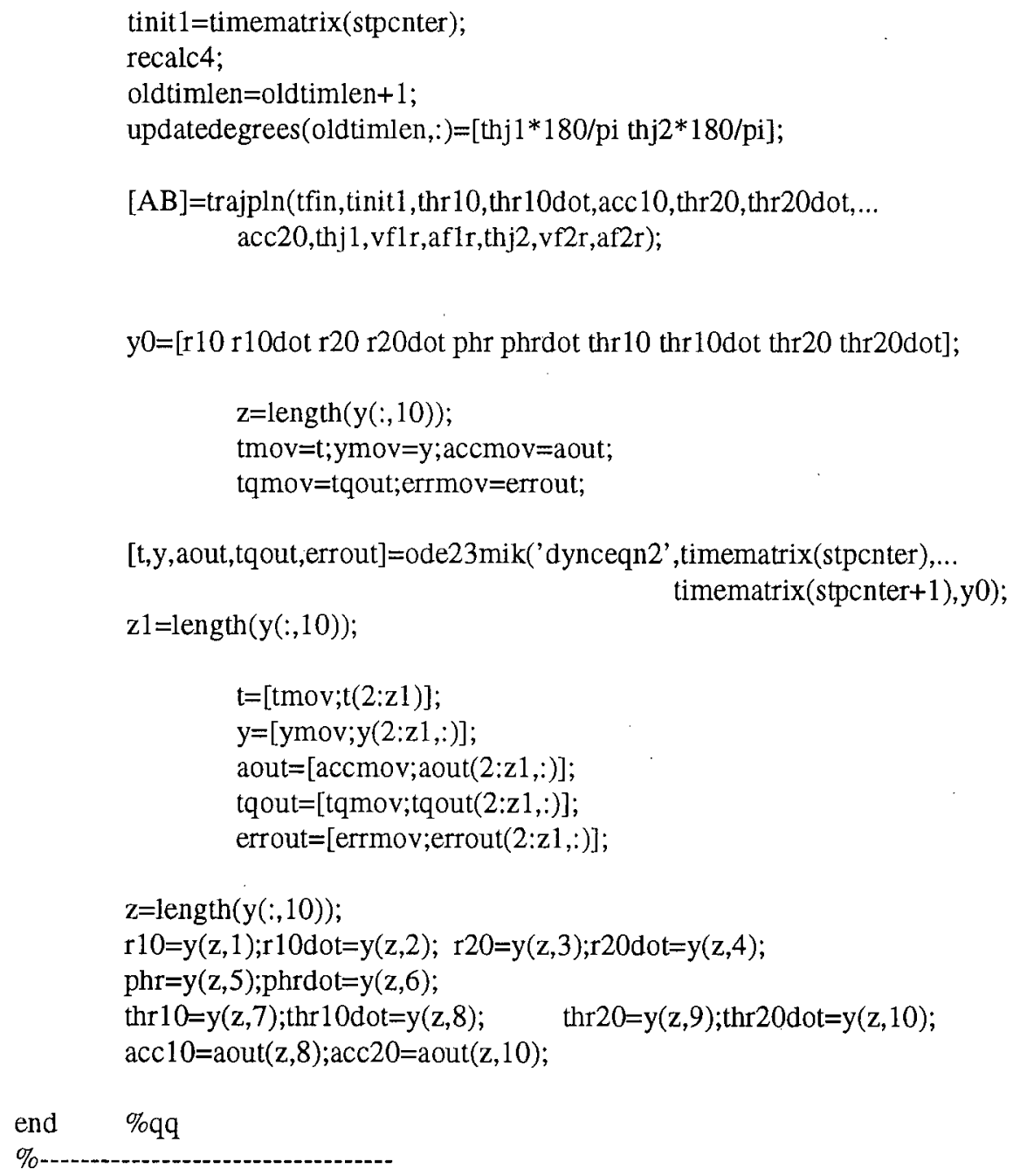

calctrj2; \% Base and link tip trajectories are calculated

errors; \% Endpoint distance and errors calculated

\%Data vectors matnum=matnum +1 ;

lerrormat (:,matnum)=lerror;

\% This next if statementis used to quit endpoint servoing after a set number of servos, $\%$ regardless of the end-effector error. It does it by setting error to zero 


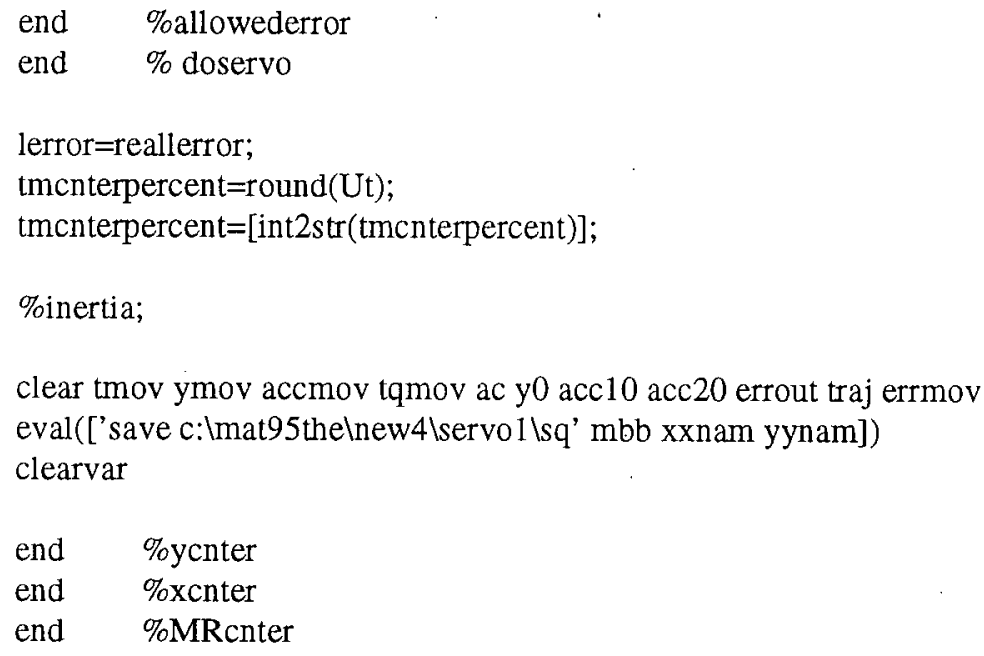




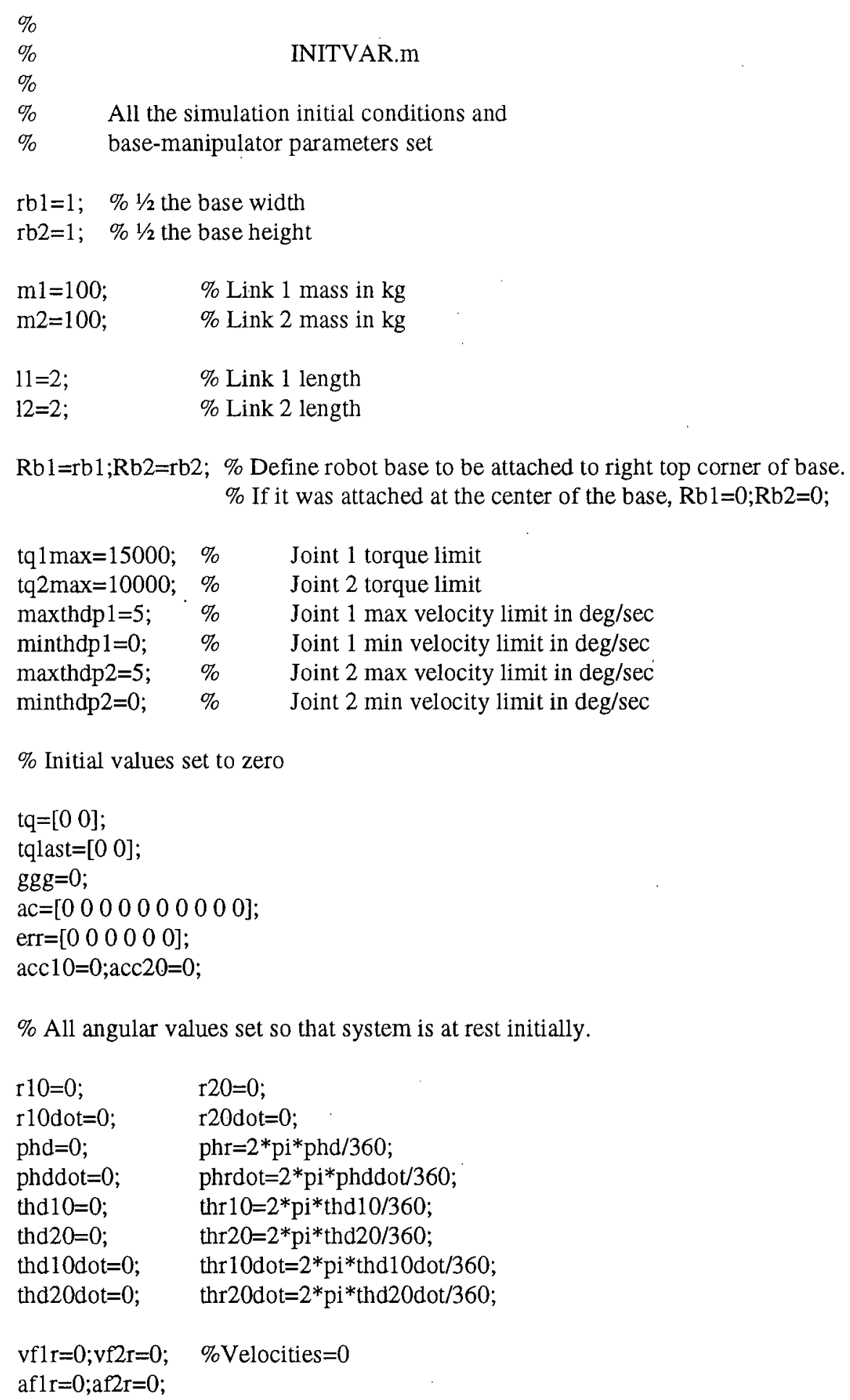

$\%$ All angular values set so that system is at rest initially. 


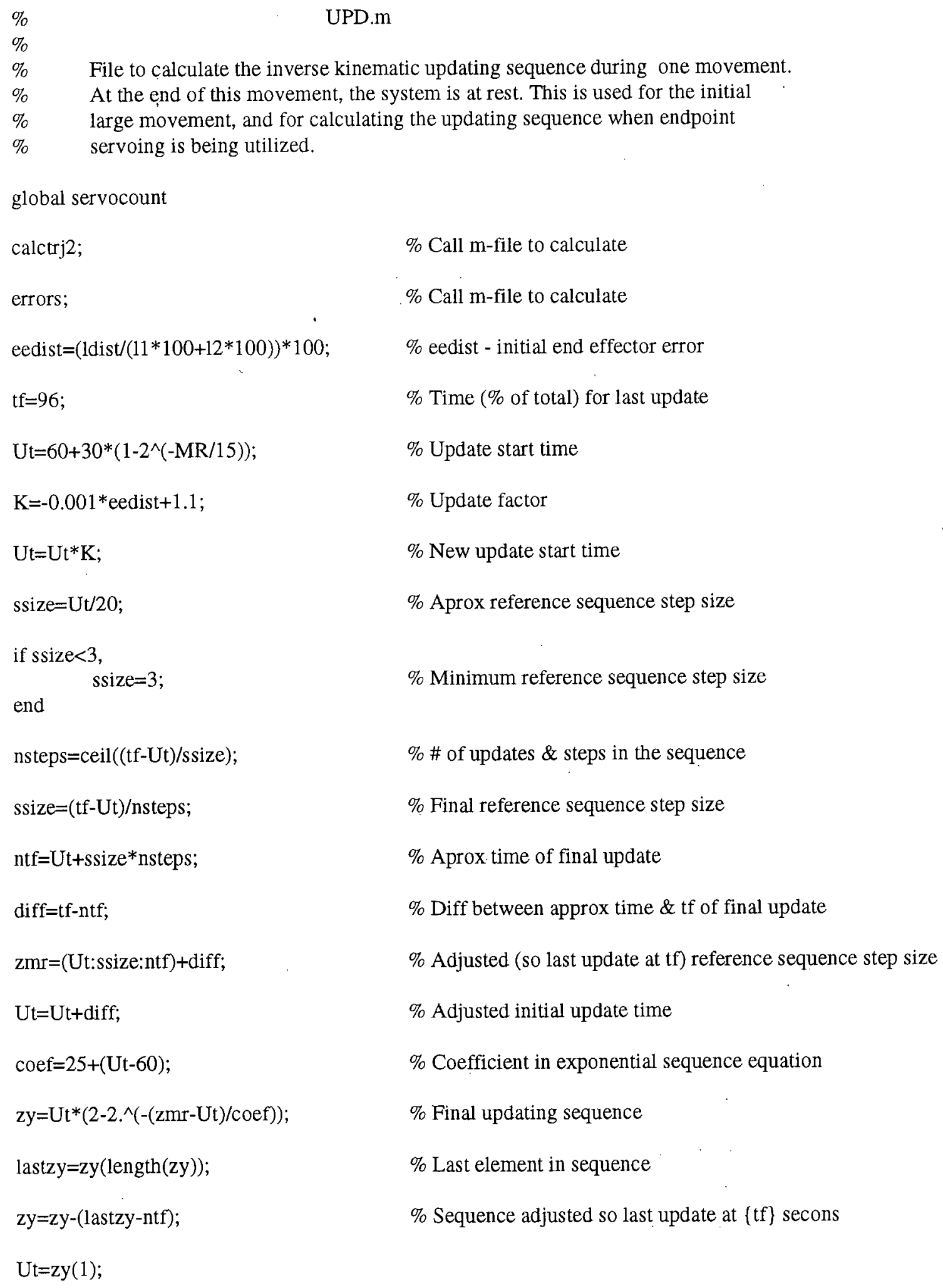




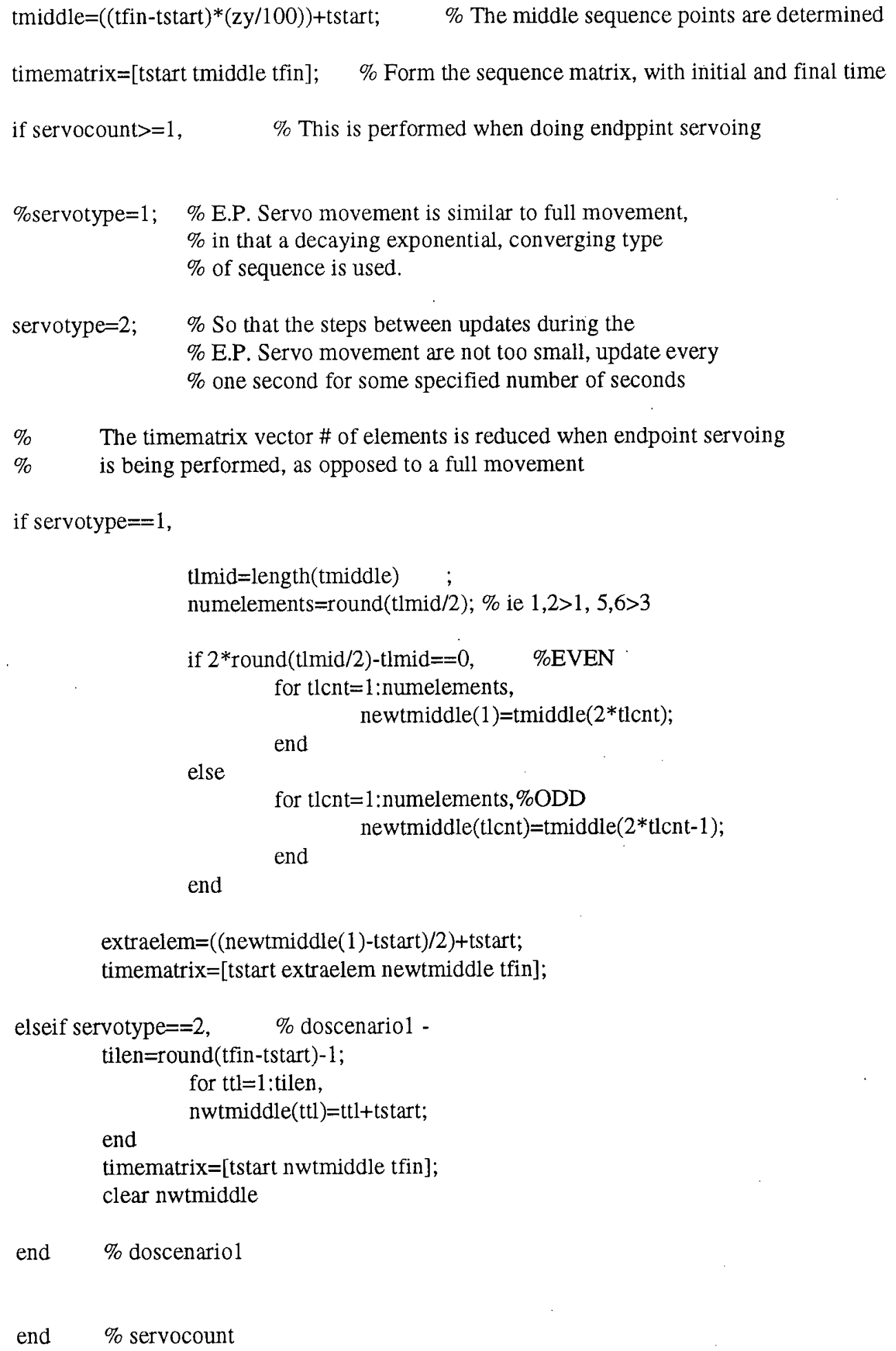


File to calculate the end-effector location in inertial (fixed) frame in the manipulator base frame, at the time when the update is occurring. The manipulator would be fixed at the start of a movement, but moving during an update. From this info., the 2-link inverse kinematics are performed, the final result being final joint setpoints to be used by the trajectory generator.

global Rb1 Rb2 1112 endeffxx endeffyy endeffx endeffy errcnt thj 1 thj2... outworkspace docgcheck doelbowcheck stpcnter allowederror servocount ... doovercomp

$\mathrm{z}=\operatorname{length}(\mathrm{y}(:, 1))$;

$\mathrm{z} 1=\mathrm{y}(\mathrm{z}: \mathrm{z}, 1) ; \quad \% \mathrm{~B}$ ase $\mathrm{X}$ at present (scalar)

$\mathrm{z} 2=\mathrm{y}(\mathrm{z}: \mathrm{z}, 3) ; \quad$ \%Base $\mathrm{Y}$ at present (scalar)

$\mathrm{z} 3=\mathrm{y}(\mathrm{z}: \mathrm{z}, 5) ; \quad$ \%Base Angle at present (scalar)

th $11=y(z: z, 7) ; \quad \% J o i n t 1$ angle at present (scalar)

th22=y(z:z,9); \%Joint 2 angle at present (scalar)

th $12=$ th $11+$ th 22 ;

$\% \quad$ Calculate the homogeneous matrix h02

h02 $=[\cos ($ th 12$)-\sin ($ th 12$) 012 * \cos ($ th 12$)+11 * \cos ($ th 11$) ; \ldots$ $\sin ($ th 12$) \cos ($ th 12$) 012 * \sin ($ th 12$)+11 * \sin ($ th 11$) ; \ldots$ $0010 ; \ldots$

$\left.\begin{array}{llllll}0 & 0 & 0 & 1\end{array}\right]$

$\%$ End effector position in manipulator base frame(frame $\mathrm{H} 2$ origin)

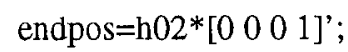

$\% \quad$ End-effector error calculated for endpoint overcompensation

xerror=endeffx-endpos $(1)$;

yerror=endeffy-endpos(2);

if doovercomp $==1$, if servocount $>=1$, corrscale $=1 ; \quad \%$ Endpoint overcompensation $\%$ variable. 1.5 causes jerkiness endeff $=$ endeff + corrscale $*$ xerror; end endeffy $=$ endeffy + corrscale $*$ yerror;

end

$\%$ Calculate the end-effector location (fixed in inertialframe) 


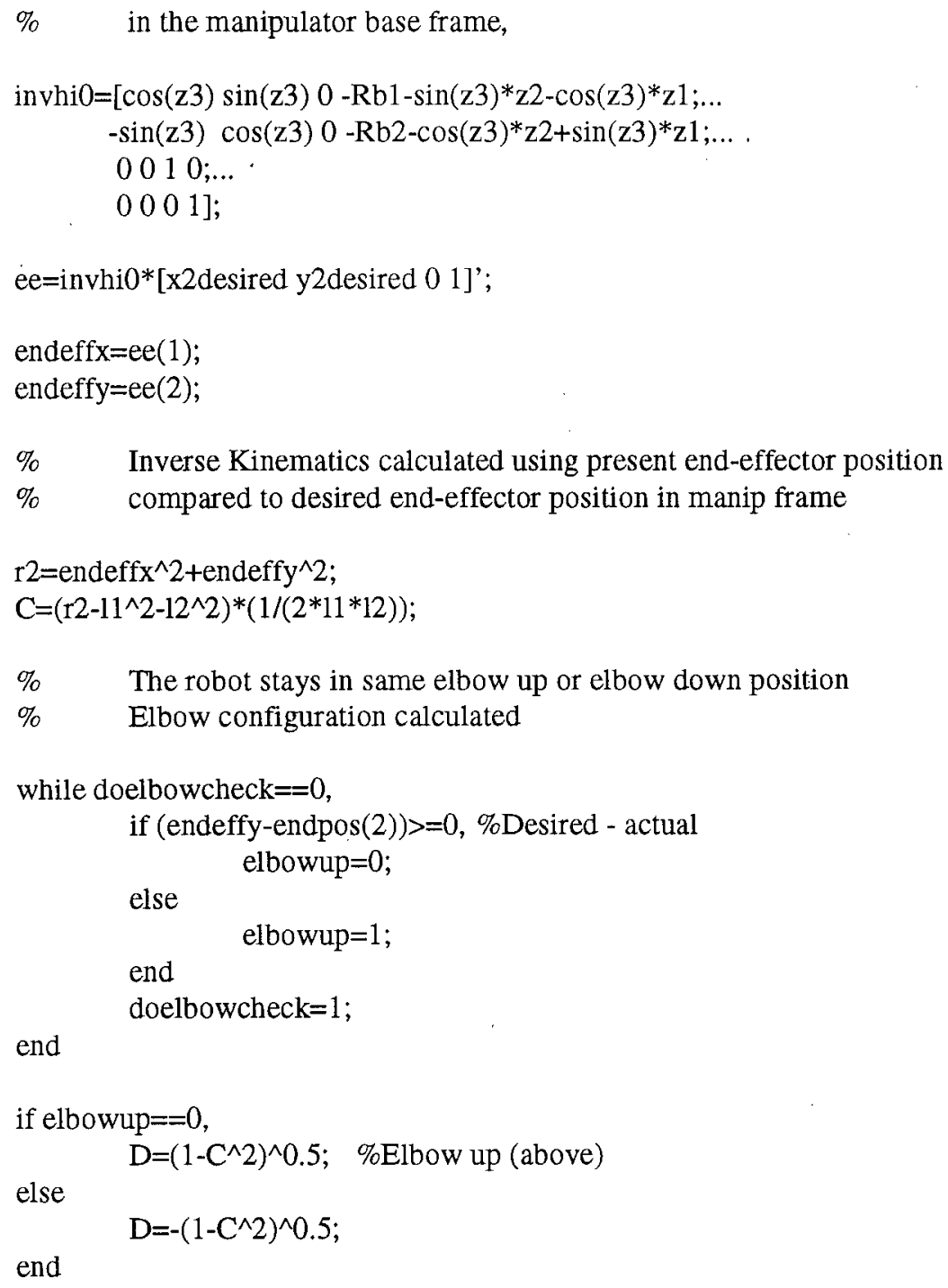


if delta1<-pi,

end

thj $1=$ thj $1+2 *$ pi;

clear $\mathrm{r} 11 \mathrm{r} 22$ phh th 11 th 22 th $12 \mathrm{c} 1 \mathrm{~s} 1 \mathrm{c} 12 \mathrm{~s} 12$

clear invhi0 h01 h02 z r2 C D thj10ld thj2old delta1 


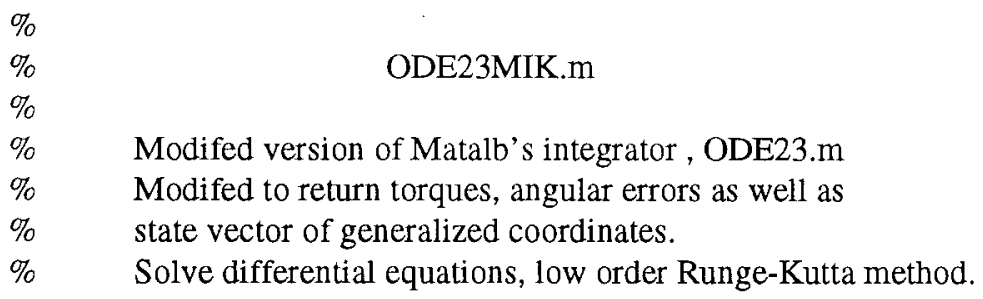

$\%$ The main loop

while $(\mathrm{t}<$ tfinal $) \&(\mathrm{t}+\mathrm{h}>\mathrm{t})$ if $\mathrm{t}+\mathrm{h}>$ tfinal, $\mathrm{h}=\mathrm{t}$ final $-\mathrm{t}$; end 


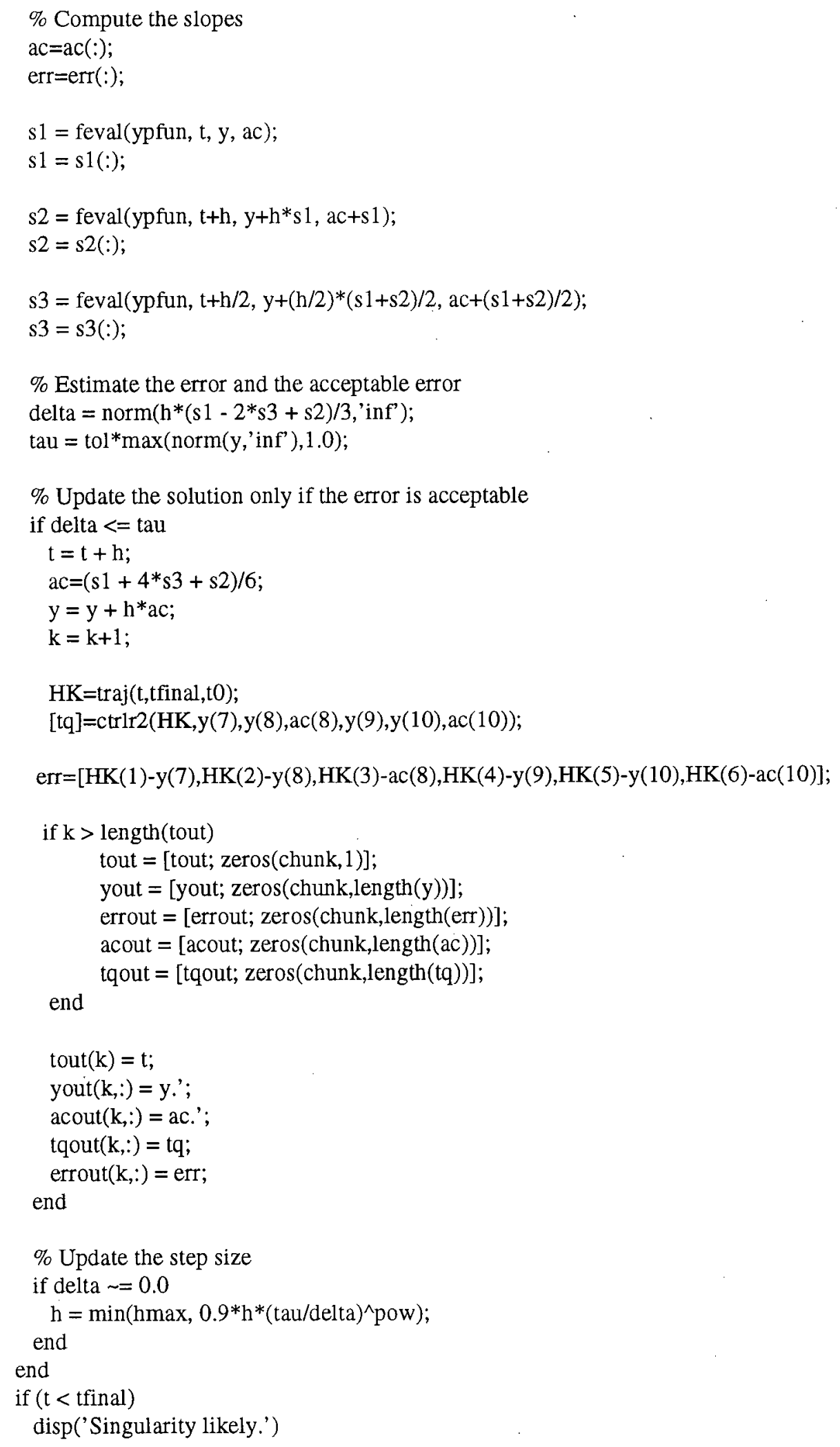




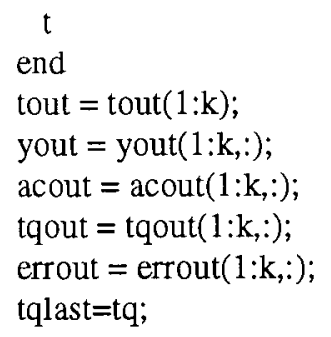




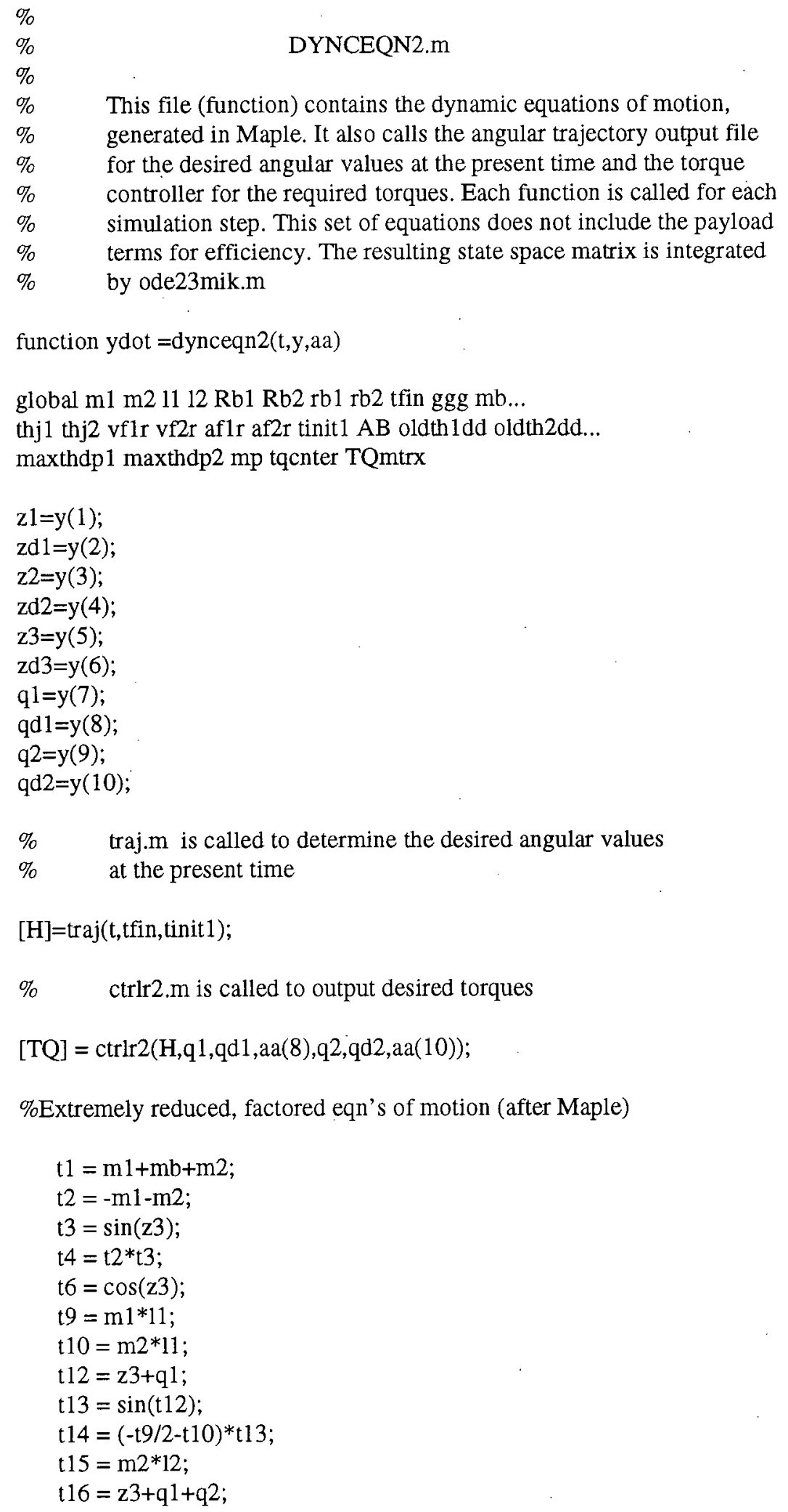




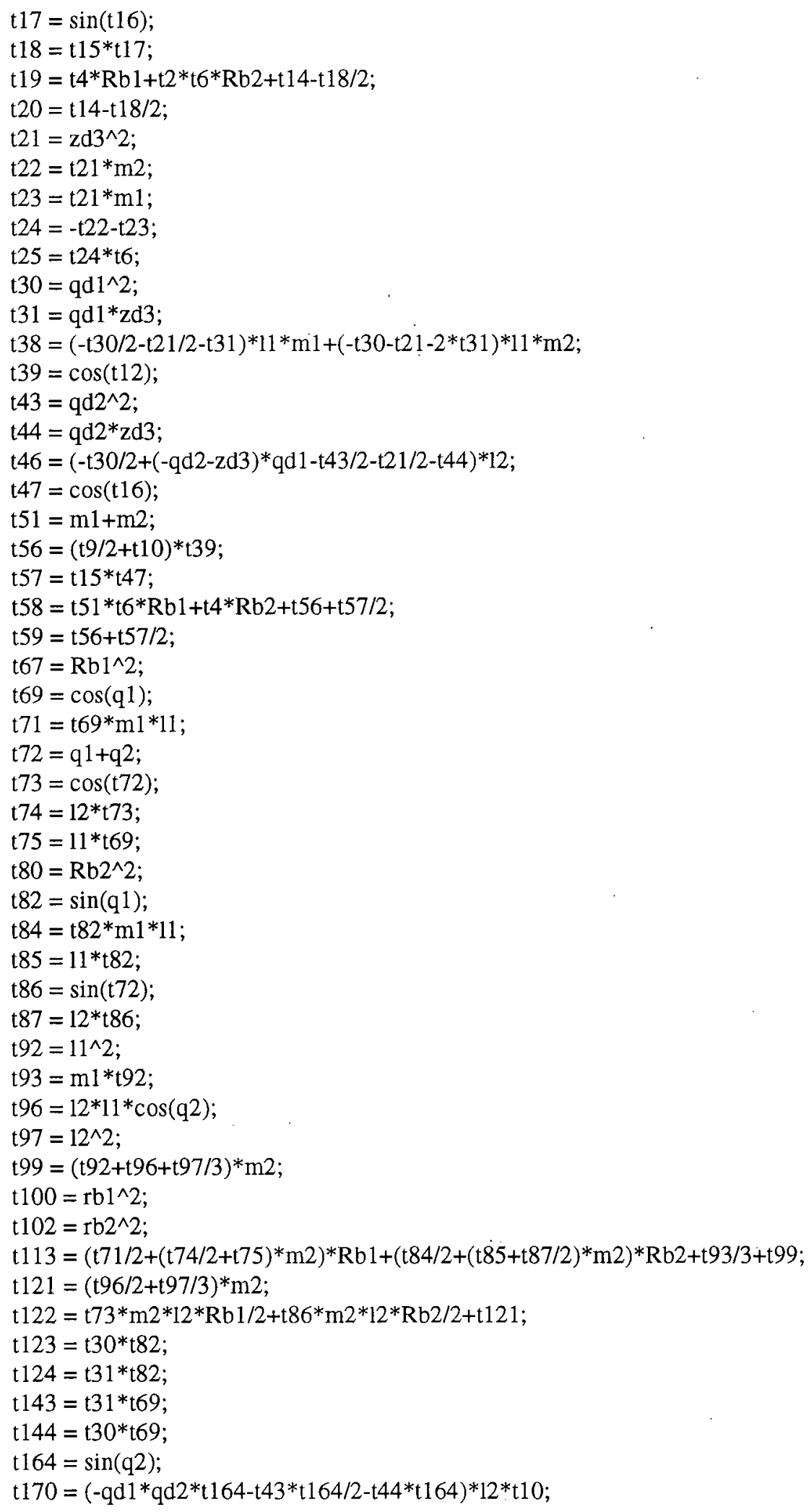




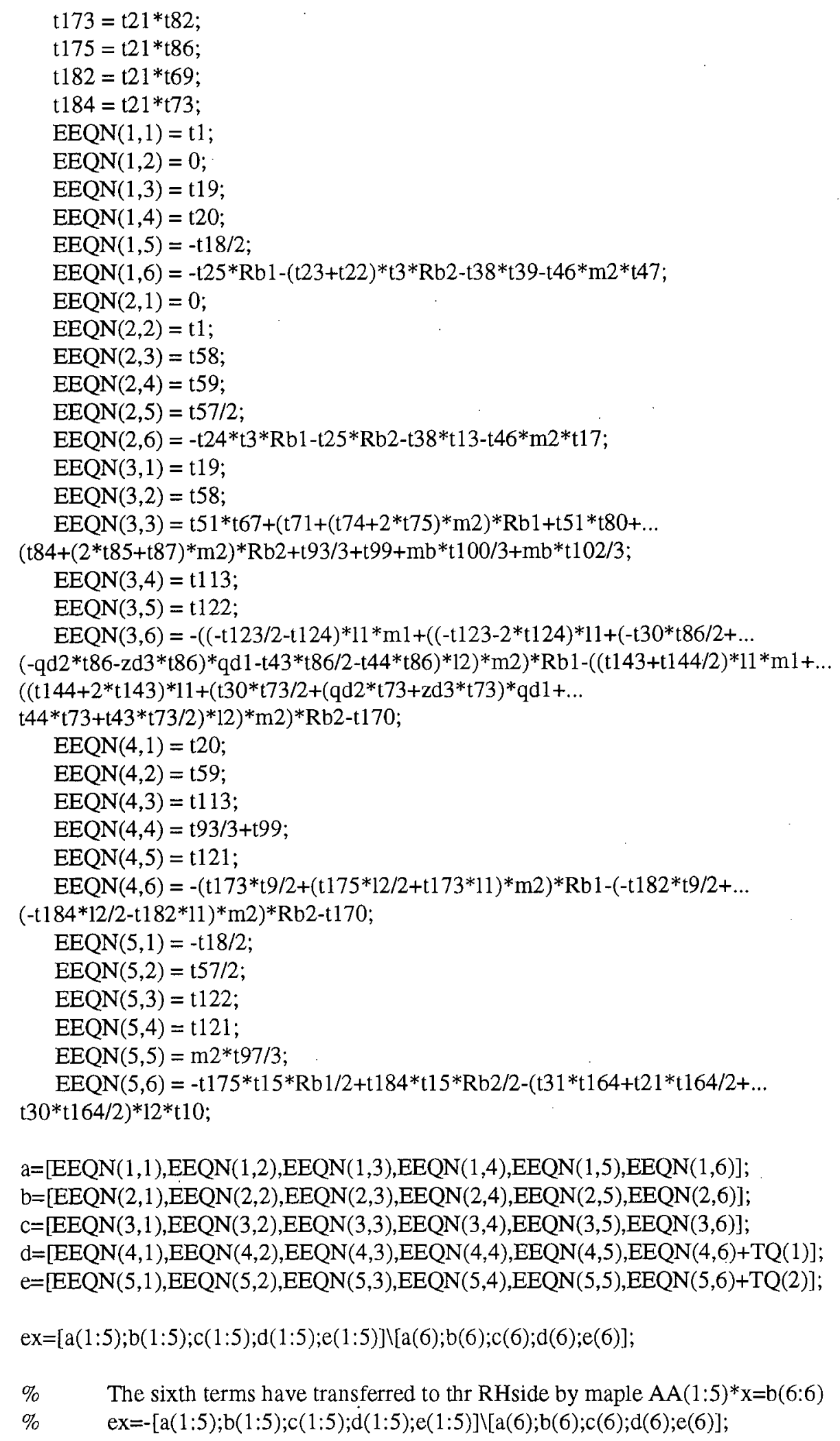


r1dotdot=ex $(1)$

r2dotdot=ex(2);

phdotdot=ex (3);

th1dotdot=ex(4);

th2dotdot=ex(5);

$\% \quad$ YDOT is the state space vector

$y d o t=[\mathrm{zd} 1 \mathrm{r} 1 \mathrm{dotdot} \mathrm{zd} 2 \mathrm{r} 2$ dotdot $\mathrm{zd} 3$ phdotdot qd1 th1dotdot qd2 th2dotdot]; 


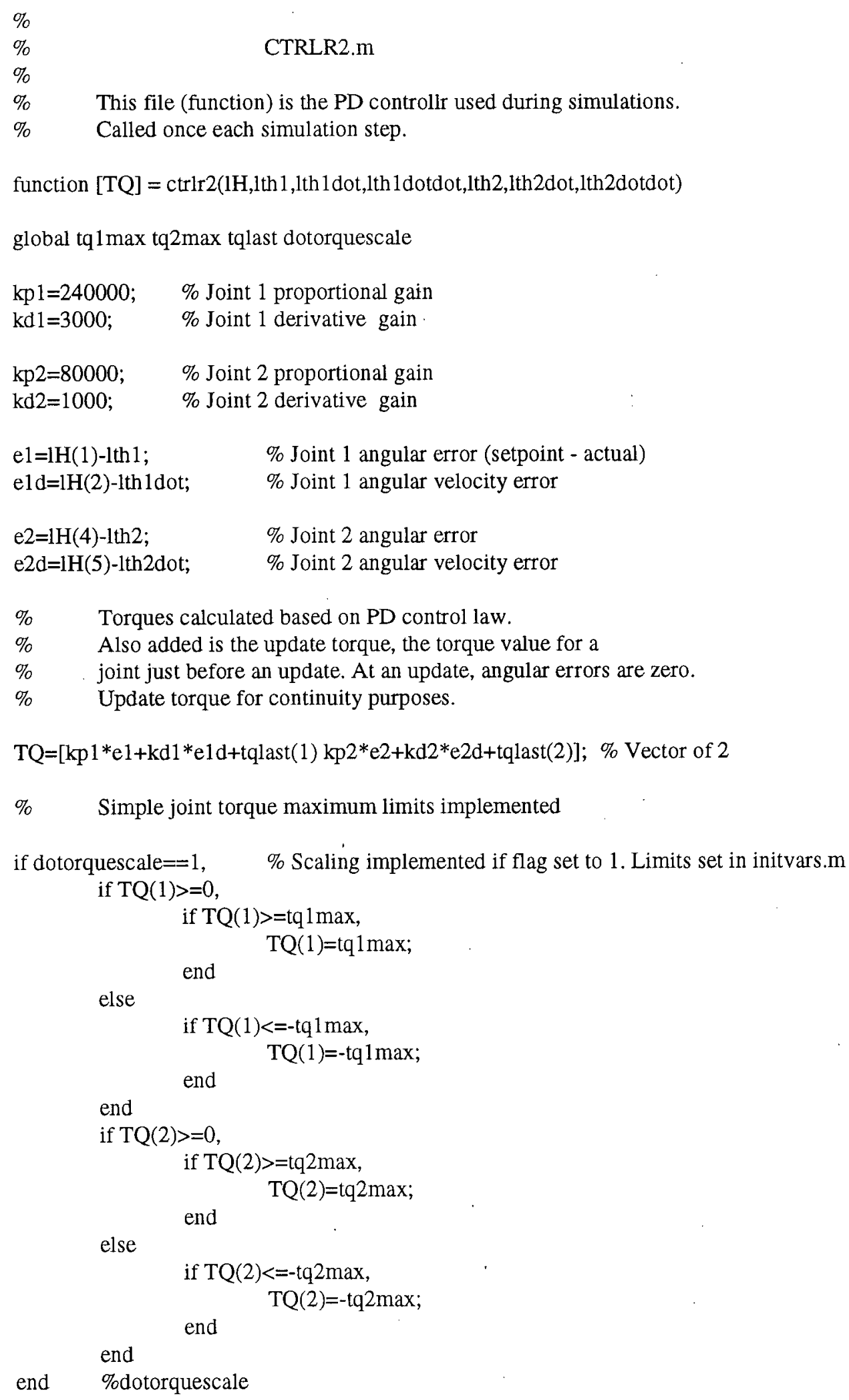




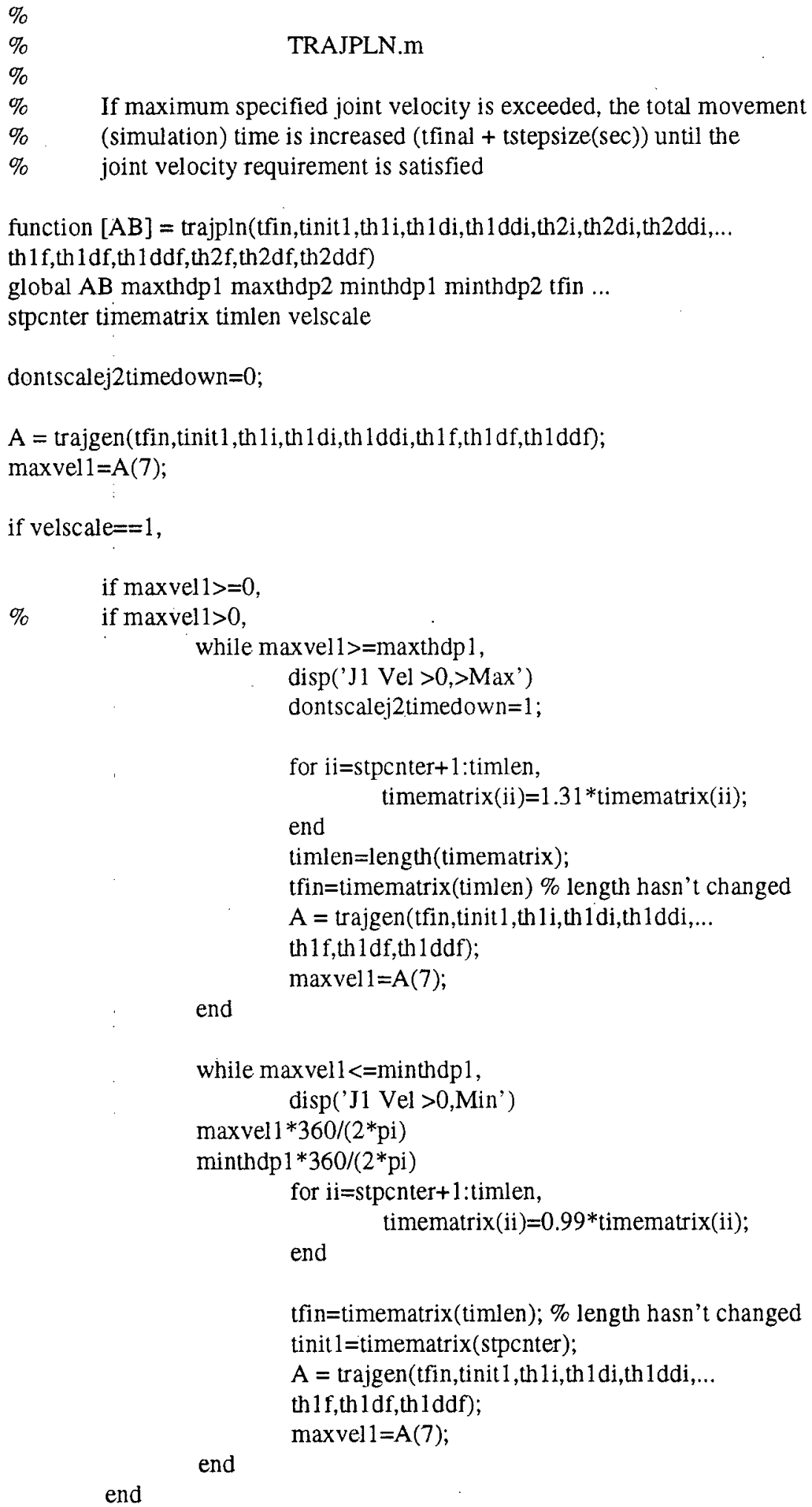




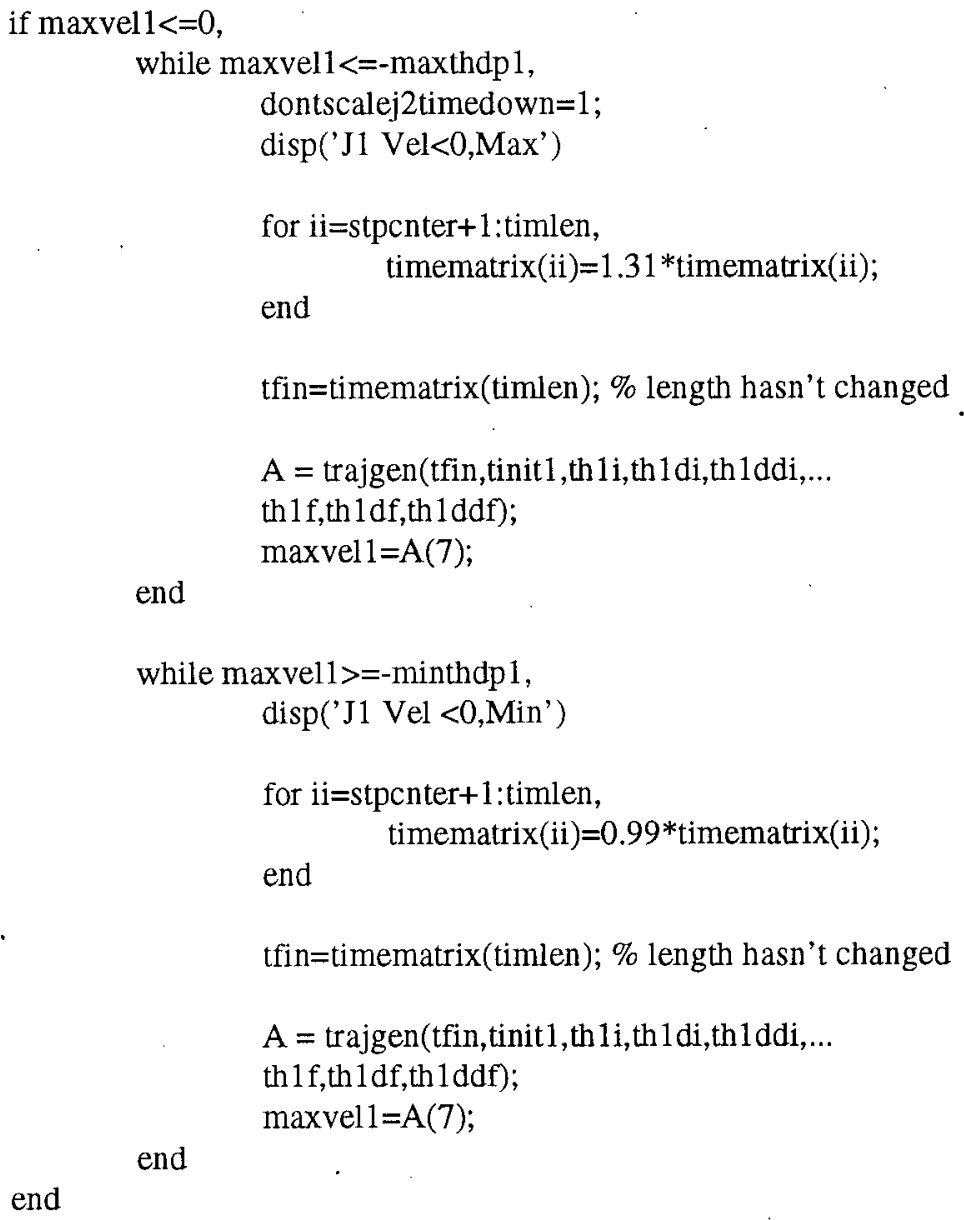

\% Possibly a new tfin, recalc tfo, etc.

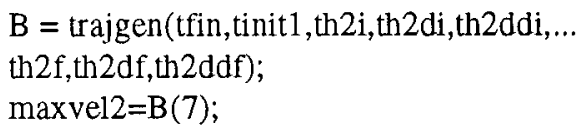




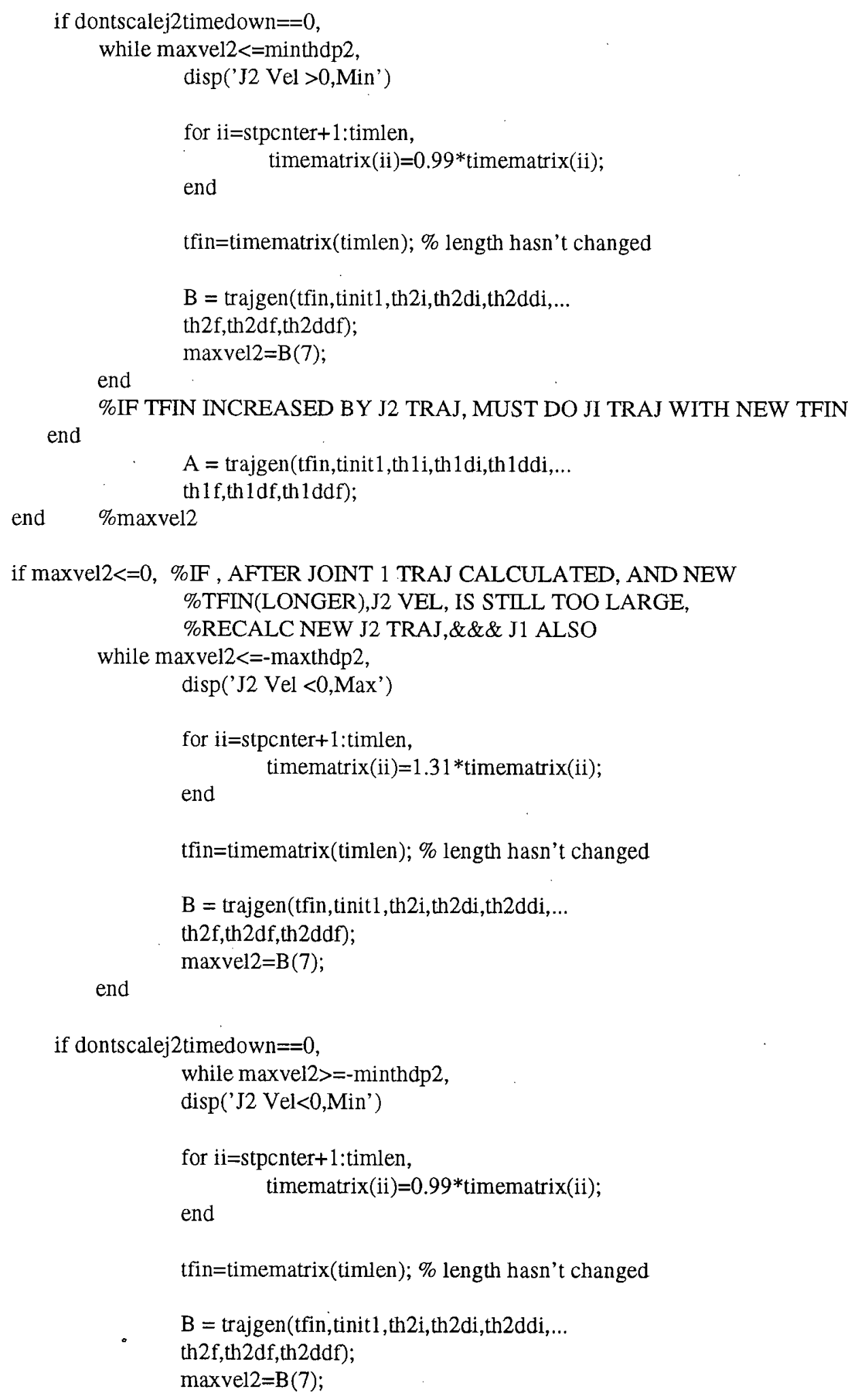




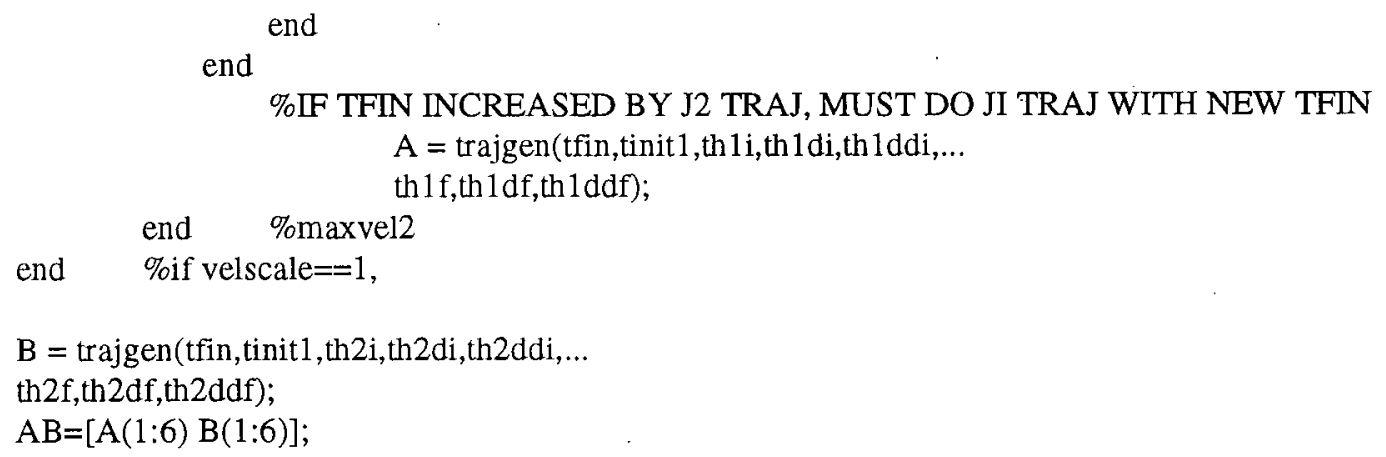




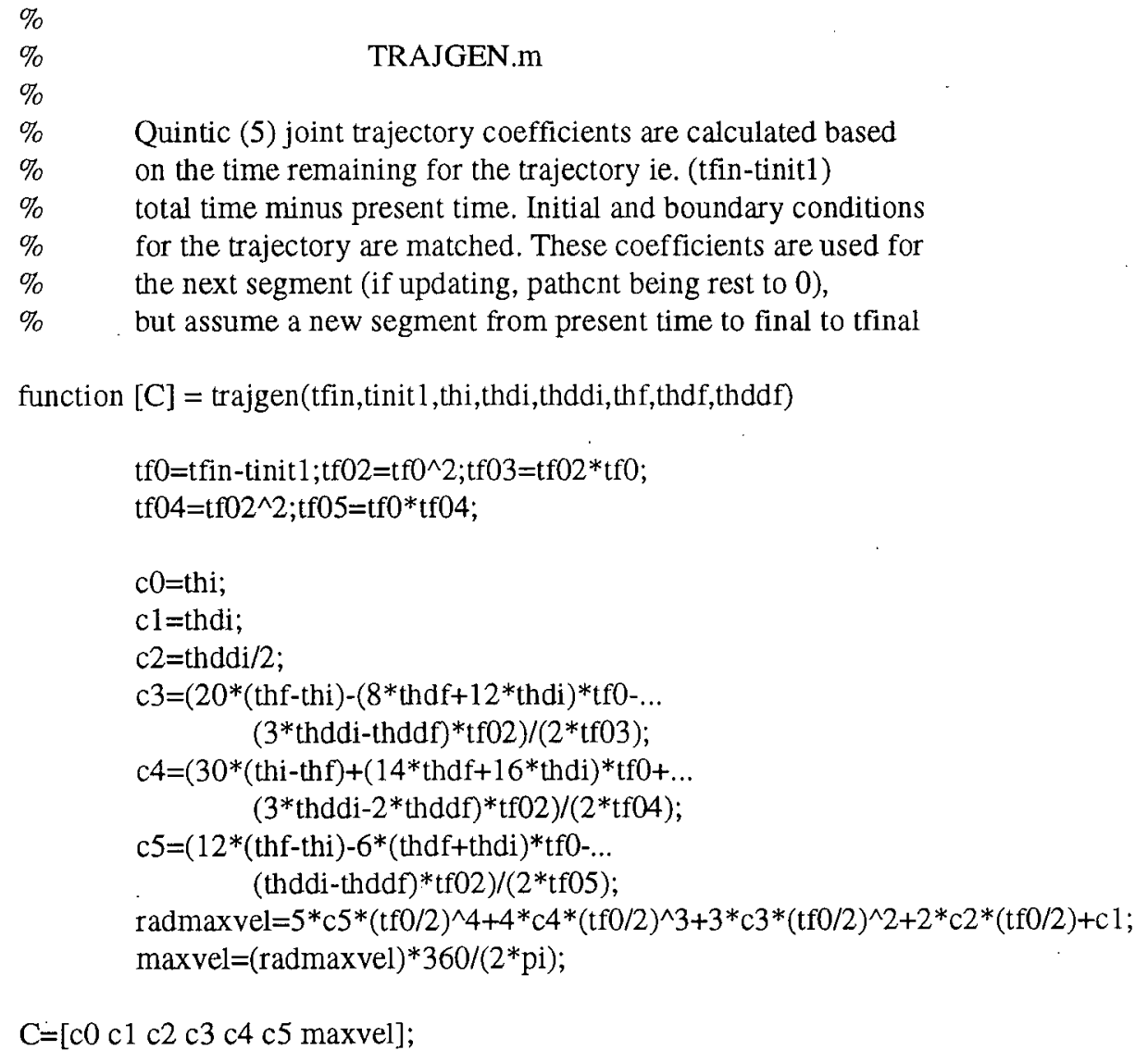




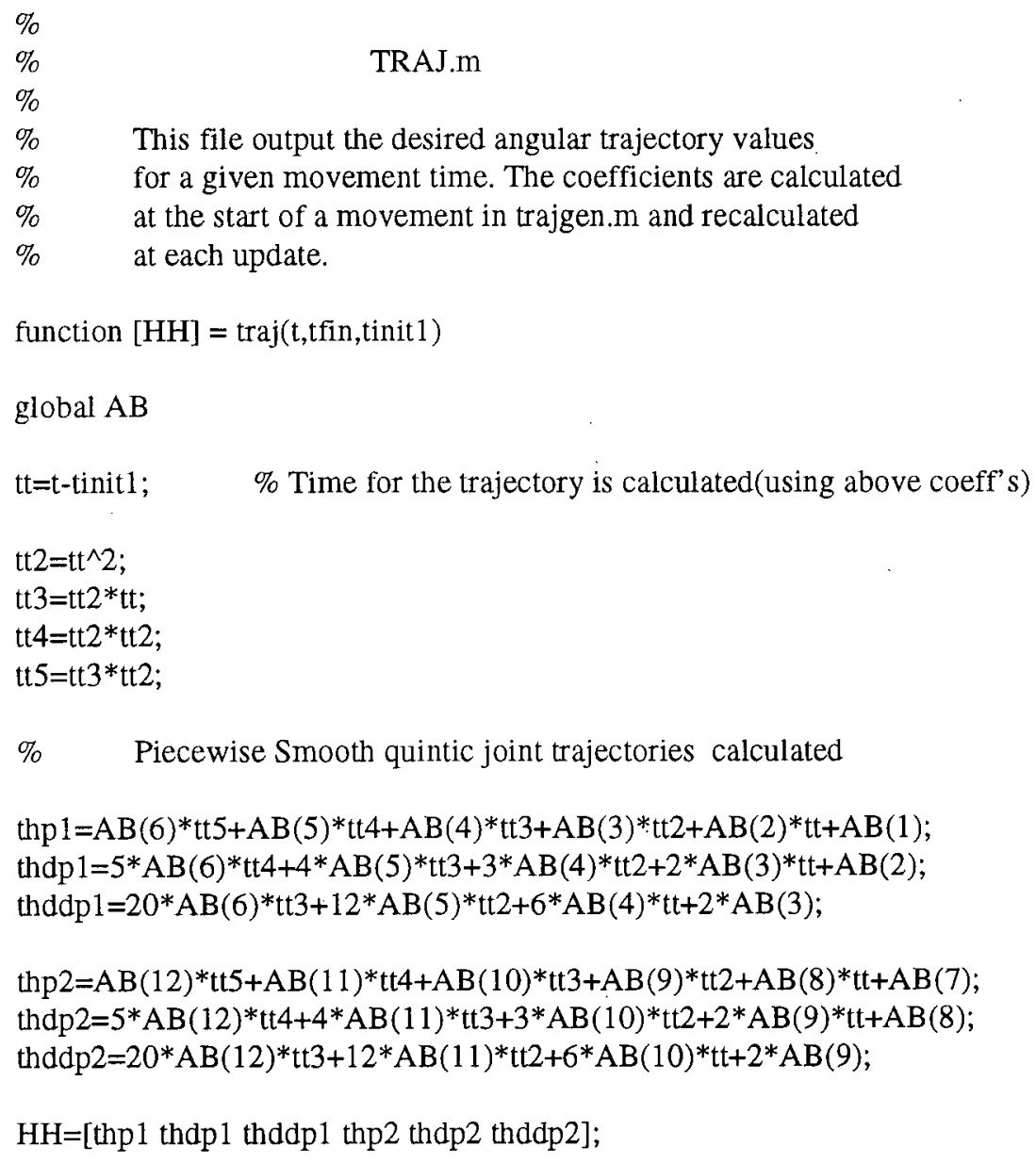




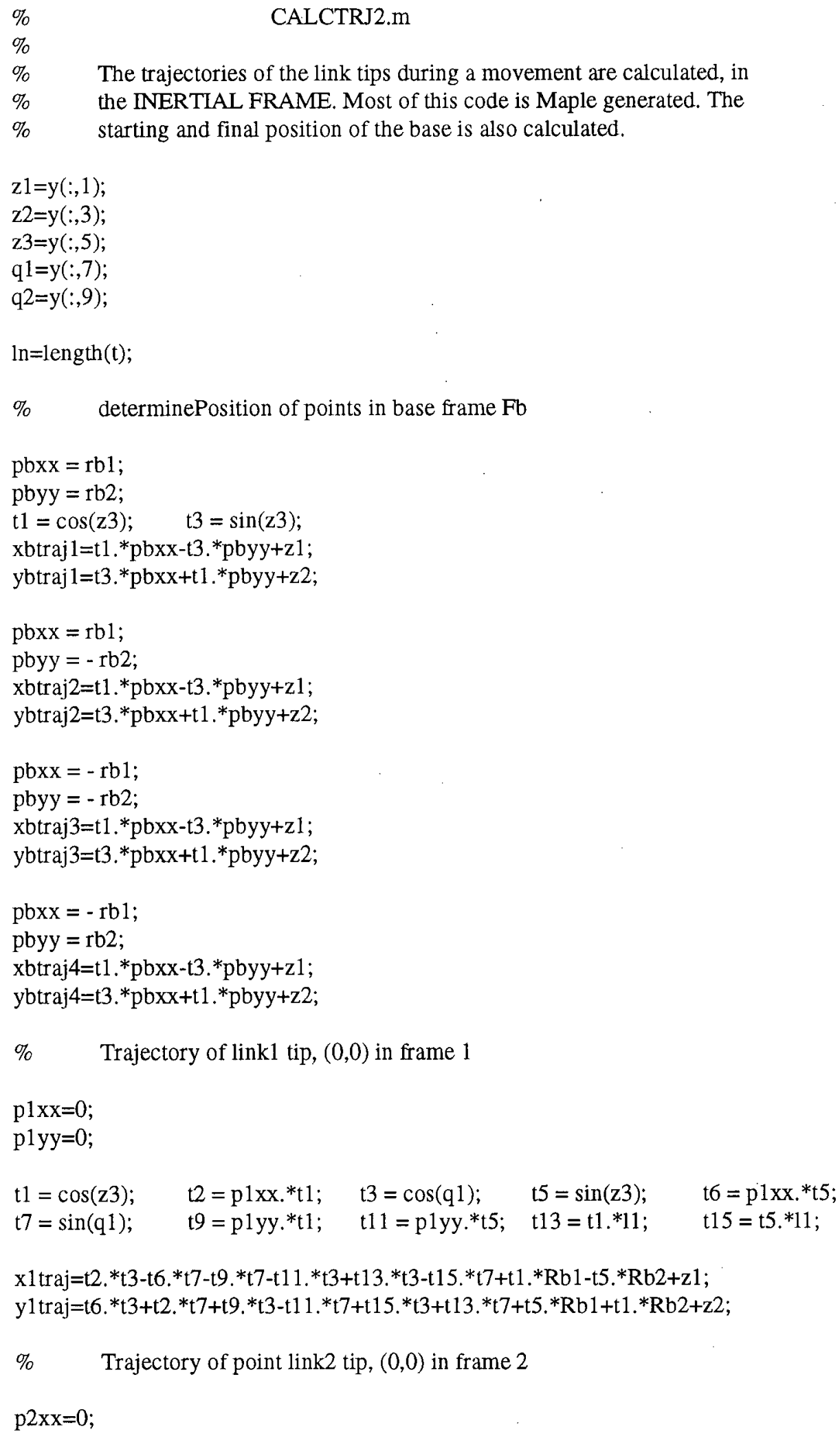




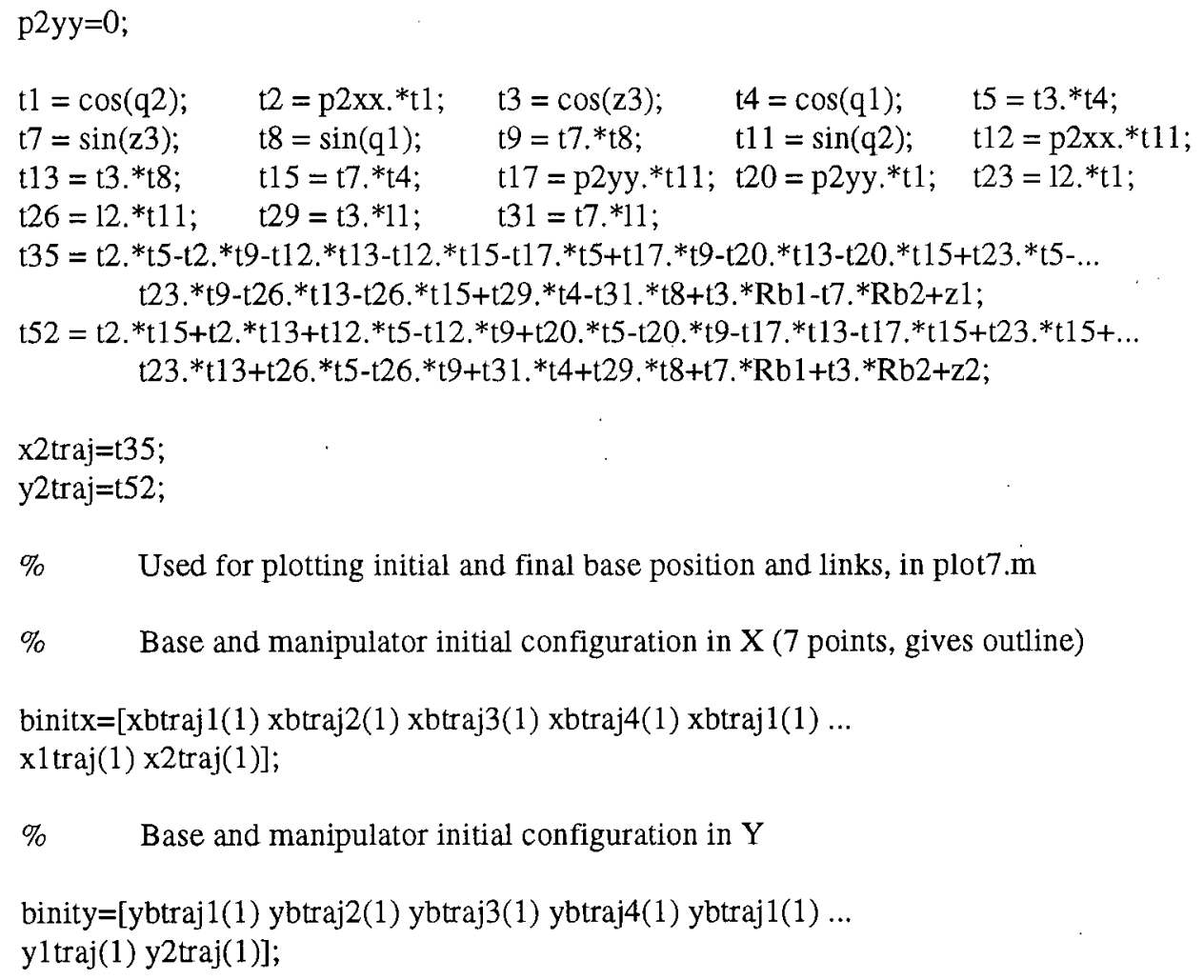


$\%$

$\% \quad$ ERRORS .m

$\%$

\% File to calculate end effector distance travelled and error

\%Endpoint Distance Travelled

ldist $=\left(\left(\operatorname{traj}(1: 1,3)^{*} 100-x 2 \text { desired } * 100\right)^{\wedge} 2+\ldots\right.$ $\left.\left(\operatorname{traj}(1: 1,4)^{*} 100 \text {-y2desired } * 100\right)^{\wedge} 2\right)^{\wedge} 0.5$;

ldist=round(ldist*100)/100;

$\%$ Endpoint Error

zly=length(traj $(:, 1))$;

lerror $=\left(\left(\operatorname{traj}(z l y: z l y, 3)^{*} 100-x 2 \text { desired }^{*} 100\right)^{\wedge} 2+\quad\left(\text { traj }(z l y: z l y, 4)^{*} 100-y 2 \text { desired } * 100\right)^{\wedge} 2\right)^{\wedge} 0.5$

lerror=round $($ lerror*100)/100;

\%Percentage Error

lperc $=($ lerror $/ \mathrm{ldist}) * 100$;

clear zly 


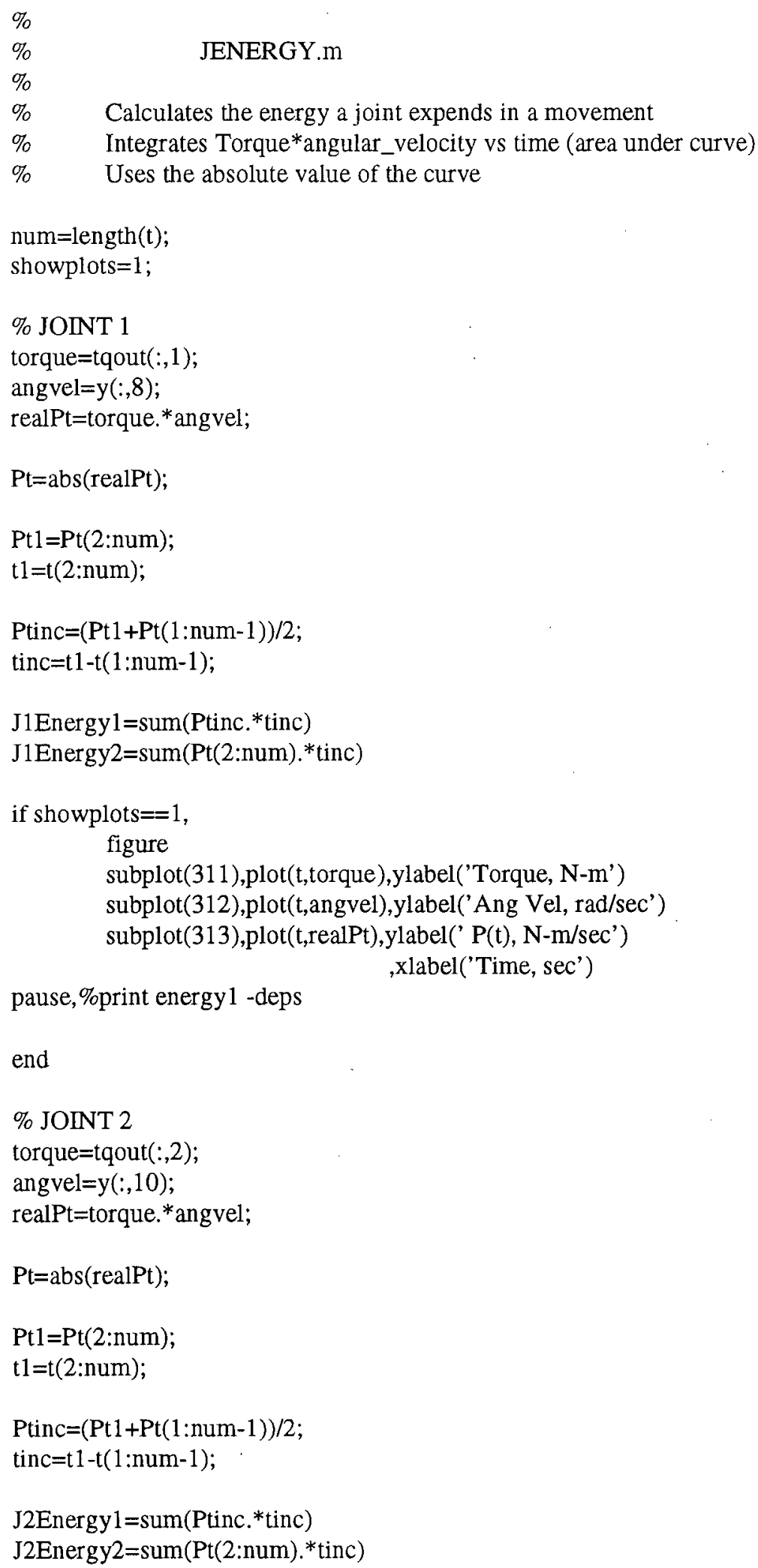

